

\title{
Nanobody-targeted photodynamic therapy: exploring cancer targets and the combination with endothelial cell targeting
}

\author{
Nanobody-doelgerichte fotodynamische therapie: \\ onderzoek naar kanker targets en de combinatie met endotheelcel \\ targeting \\ (met een samenvatting in het Nederlands)
}

\section{Proefschrift}

ter verkrijging van de graad van doctor aan de Universiteit Utrecht op gezag van de rector magnificus, prof. dr. H.R.B.M. Kummeling, ingevolge het besluit van het college voor promoties in het openbaar te verdedigen op woensdag 13 januari des ochtends te 10.30 uur

door

\section{Vida Mashayekhi}

geboren op 20 september 1987 te Mianeh, Iran 
Promotor:

Prof. dr. A.S. Akhmanova

Copromotoren: Dr. S. Oliveira

Dr. P.M.P. van Bergen en Henegouwen

The research presented in this thesis was funded by the European Research Council (ERC) under the European Union's Horizon 2020 research and innovation program (grant 677582). 
Nanobody-targeted photodynamic therapy: exploring cancer targets and the combination with endothelial cell targeting.

$\mathrm{PhD}$ thesis with a summary in Dutch

Department of Biology, Utrecht University, The Netherlands

ISBN: 978-94-6423-090-1

Printed by: Proefschriftmaken

Cover design: Kamal Tehrani

layout: Vida Mashayekhi, Kamal Tehrani 
To my Mom and Dad,

who have encouraged me to go on every adventure in my

life, especially this one 
Table of contents

Chapter 1 Introduction

Chapter 2 Nanobody-targeted photodynamic therapy selectively kills viral GPCR- 41 expressing glioblastoma cells

Chapter 3 VHH-photosensitizer conjugates for targeted photodynamic therapy of Met- 75 overexpressing tumor cells

Chapter 4 Acute cellular and vascular responses to photodynamic therapy using 95 EGFR-targeted nanobody-photosensitizer conjugates studied with intravital optical imaging and magnetic resonance imaging

Chapter 5 Vascular targeted photodynamic therapy: a review of the efforts towards 129 molecular targeting of tumor vasculature

Chapter 6 Dual targeting of endothelial and cancer cells potentiates in vitro nanobody153 targeted photodynamic therapy

Chapter 7 General Discussion

Appendices English summary

Nederlandse samenvatting

List of publications

Curriculum Vitae

Acknowledgments 


\section{Chapter 1}

\section{Introduction}

The chapter is adapted from the published review manuscript:

Oncologic Photodynamic Therapy: basic principles, current clinical status and future directions

Demian van Straten, Vida Mashayekhi, Henriette S. de Bruijn, Sabrina Oliveira and Dominic J. Robinson

Cancers, 2017; 9(2):19

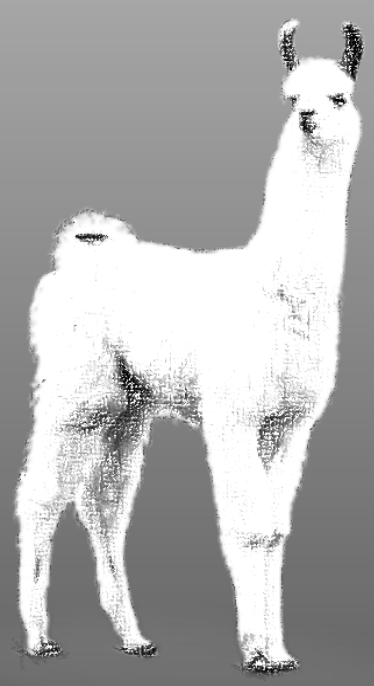




\section{Cancer therapy}

The main goal of cancer therapy is the eradication of malignant cells, ideally

without affecting normal cells. Traditional and well-known therapies consist of surgery, chemotherapy and radiotherapy, which can be used alone or in combination to get better results. However, each approach has its own drawbacks. Radiotherapy has been used for cancer treatment for a long time. In this approach, radiation is targeted to a tumor by only irradiating a certain part of the body. Ionizing radiation works by damaging the DNA of cancerous cells leading to an inhibition of the cell cycle and consequently apoptotic cell death. Although radiotherapy is known as a local treatment, it cannot distinguish cancer from healthy cells. For several decades, major efforts have been made to develop more effective approaches to improve the therapeutic efficacy and further decrease side effects. Photodynamic therapy (PDT) is a promising minimally invasive approach that is used for the local treatment of several cancers and has been shown to be more selective among the other therapeutic approaches [1]. It is less invasive than surgical procedures and side effects usually last for a shorter period than chemotherapy or radiotherapy. PDT is based on a photochemical reaction between a light activatable molecule or photosensitizer (PS), light that is usually in the visible spectrum, and molecular oxygen. These three components are harmless individually, but in combination result in the formation of reactive oxygen (ROS) species [2] that are able to directly induce cellular damage to organelles and cell membranes, depending on where they are generated [3]. PDT is a two-stage procedure consisting of the intravenous, intraperitoneal or topical administration of a PS, or PS precursor, followed by local illumination. This two-stage procedure significantly reduces side effects, as the harmless PS is activated only via the directed illumination, resulting in local tissue destruction.

\section{Principles of PDT}

\subsection{Photodynamic reactions}

Although the precise mechanism of action of PDT is an ongoing topic of investigation, its molecular effects are accepted to be based on the reaction of a light activated PS with other molecules, creating radicals [4]. Illumination of a PS leads to the absorption of a photon and promotes the PS to its excited singlet state, or $\mathrm{S}_{1}$, in which an electron is shifted towards a higher-energy orbital (Figure 1). From this unstable and typically short- 
lived state, the PS can return to its ground state $S_{0}$ by converting its energy into heat or fluorescence, a feature which can be used for the purposes of diagnostics and optical monitoring [5]. Alternatively, intersystem crossing can occur resulting in the population of the PS as excited triplet state $T_{1}$. In this $T_{1}$ state, the PS can transfer its energy by phosphorescence or by colliding with other molecules to create chemically reactive species via two types of reactions $[4,6]$. $T_{1}$ can react with a number of organic substrates or solvents and transfer an electron or proton to form radical anion or cation species, respectively. Typically, the PS reacts with an electron donating substrate to and subsequently reacts with oxygen to form superoxide anion radicals. This is called a type I reaction. In a type II reaction, $\mathrm{T}_{1}$ reacts directly with ground state oxygen ${ }^{3} \mathrm{O}_{2}$, transferring energy to form singlet oxygen ${ }^{1} \mathrm{O}_{2}$, which is one of the highly reactive oxygen species (ROS) [6]. The exact molecular mechanisms of these photochemical reactions have been described in detail elsewhere [7].

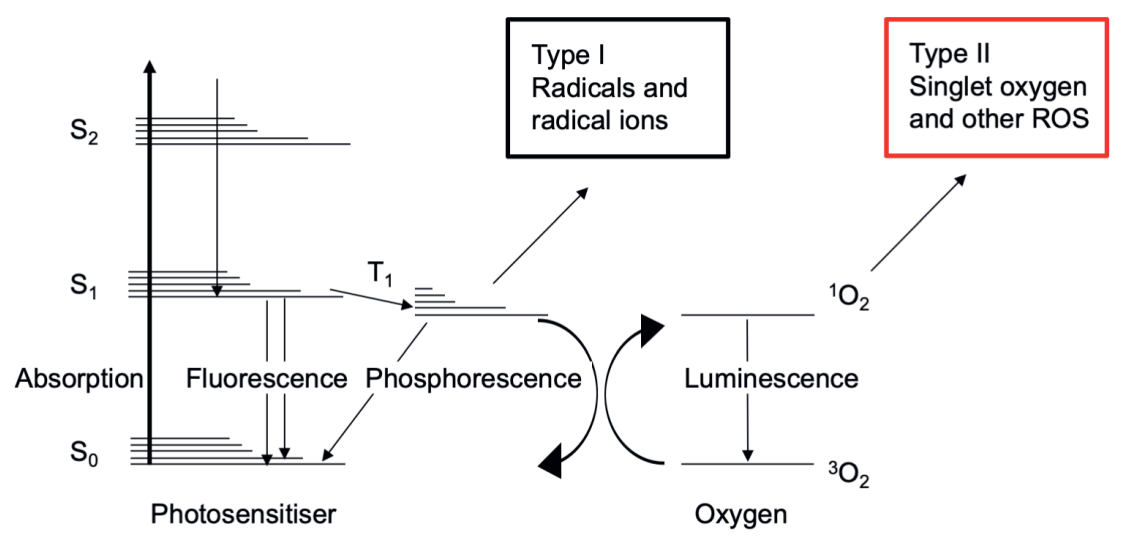

Figure 1. Schematic representation of type I and type II reactions following photosensitizer activation upon illumination. $\mathrm{S}_{0}$ : ground state of the PS molecule; $\mathrm{S}_{1}$ : excited singlet state of the PS molecule; $\mathrm{T}_{1}$ : triplet excited state of the PS molecule; ${ }^{3} \mathrm{O}_{2}$ : ground state oxygen; ${ }^{1} \mathrm{O}_{2}$ : singlet oxygen

The production of singlet oxygen and superoxide anions will result in cytotoxicity as both products can directly react with and damage biomolecules such as lipids, proteins and nucleic acids [3]. Singlet oxygen is highly reactive, its lifetime is in the order of $40 \mathrm{~ns}$ and has a maximum action radius of about $20 \mathrm{~nm}[8,9]$. In addition, it has been reported that some species such as hydrogen peroxide and superoxide anion can diffuse several micrometres [10]. Besides oxygen species, diffusion of nitrogen species, such as 
peroxynitrite radicals, has been shown over several micrometres $(5-20 \mu \mathrm{m})$ [11]. This short action radius together with localized PS activation by only illuminating target tissues, theoretically, renders PDT very specific and controllable. It also means the localization of the PS influences the site of action of PDT at the subcellular level [9].

\subsection{Mechanism of action of PDT at cellular level}

The cellular response to photodamage is strongly dependent on multiple factors of which PS localization is the most important [4]. The intracellular site of action is PS dependent and plays a significant part in the fate of the cell. In a study comparing the importance of the subcellular location of PS in inducing cell death, the PS Cristal Violet (CV) that was less efficient at producing radicals was equally efficient in inducing cell death as Methylene Blue (MB), that produced 10 times as much radicals. This is predominately due to the subcellular location of the PS, as MB is localized in the cytosol and lysosomes, while $\mathrm{CV}$ is present in mitochondria, suggesting localization is more important rather than the amount of radicals formed [12]. Depending on its characteristics, a hydrophobic PS will generally localize in the hydrophobic inner layer of the membrane such as the membrane from the plasma membrane, lysosomes, mitochondria, Golgi apparatus or endoplasmic reticulum (ER) [13]. The cytoskeleton and cell adhesion components have also been described as PS targets [14]. Even though photodynamic action may affect many different targets, three main mechanisms of photodamage induced cell death have been described: apoptosis, necrosis and autophagic cell death. The ability of PDT to activate multiple cell death pathways circumvents the problem of apoptosis resistant cells in tumors, which can be a major obstacle for other cancer therapeutics [15]. Overall, determining the outcome of PDT on a cellular level is complex. Nevertheless, some general themes can be observed [16]. With high doses of PDT or PS localization to the plasma membrane, necrosis is the dominant form of cell-death. With mild PDT and damage to the mitochondria or anti-apoptotic components, apoptosis is triggered. With low PDT induced damage to organelles, autophagy is initiated to try and repair the damage. However, when the protective capacity of autophagy is overwhelmed or compromised due to for instance lysosomal damage, autophagy can induce cell death. Elucidating the exact effects of PSs and subsequent responses on a cellular level is crucial to understand the effect of PDT. 


\subsection{Mechanism of action of PDT at tumor level}

The phototoxic effect of PDT, as currently employed in the clinic, is in general minimally tumor selective. PSs are taken up by both healthy and tumor cells. In general, normal tissues are capable of eliminating or clearing the PS over time, while tumor tissues cannot, due to non-existing lymphatics. This leads to some retention of PS in tumor tissue, which combined with the localized activation by specific illumination, gives PDT some selectivity. Factors that affect the preferential accumulation of PS towards tumor neoplastic tissue are complex and multiple theories exist based on several mechanisms [17]. A popular theory which applies to all PSs is based on the morphological differences between healthy and tumor tissues. Due to the rapid and uncontrolled growth of tumor cells, solid tumors have abnormal, unorganised vasculature with a defective inner lining. Consequently, tumor endothelium is leaky, enabling therapeutics (mainly (lipo)protein associated PS) to extravasate into the extravascular space. Moreover, they are retained longer compared to healthy tissues due to the impaired lymphatic drainage in tumor tissue. This phenomenon is called the Enhanced Permeability and Retention (EPR) effect and is often utilized in cancer therapeutics [18]. Other theories attribute localization to increased expression of certain receptors on tumor cells, decreased intratumoral $\mathrm{pH}$ or tumor associated macrophages (TAM) that phagocytose PS molecules [19]. These mechanisms are not fully understood and are heavily influenced by PS characteristics (hydrophobicity/-philicity), tumor type and dosage, amongst other aspects [19]. PDT is considered to have three main mechanisms of tumor destruction. Due to the localization and activation of the PS inside the tumor tissue, generated ROS can directly kill the malignant tumor cells. Secondly, PDT can affect tumor vasculature thereby compromising the supply of oxygen and essential nutrients. The third mechanism is the PDT activated immune system, inducing an inflammatory and immune response against tumor cells.

\subsubsection{Direct ROS effects}

Just as the subcellular PDT site of action is important for the effect on the cell, the cellular site of action is important for the effect on the tumor. Tumors are made up of the parenchyma consisting of the malignant cells and the stroma, which is the supportive, vascularised tissue. Tumor stroma includes plasma protein-rich interstitial fluid, structural proteins, fixed connective tissue cells and inflammatory cells and can make up as much as $90 \%$ of the tumor mass [20]. All solid tumors require stroma to grow since it supports the 
blood vessels that provide nutrients and oxygen and regulate waste disposal [21]. One can imagine different PDT outcomes depending on what part of the tumor is affected. The most direct form of tumor damage done by PDT is the killing of parenchyma cells. PSs that accumulate in tumor parenchyma have the obvious effect of cell damage and subsequent tumor cell apoptosis, necrosis or autophagy dependent cell death. However, early studies already showed that direct destruction of neoplastic cells alone is not enough for tumor cure [4]. This led to the belief that damage to tumor stroma may play a major role in PDT efficacy, a hypothesis also recognized in other fields of cancer therapeutics [22]. The interactions between tumors and their extracellular matrix play a critical role in tumor cell growth, motility and invasiveness indicating the potential effect of modulating these interactions $[23,24]$. PDT induced damage to structural proteins such as integrins could disrupt essential stromal-tumor signalling [23]. Also, the destruction of stromal fibroblasts inhibits tumor progression and can increase therapeutic response by loosening the tumor extracellular matrix [25].

The direct cell killing effect of PDT, on both tumor parenchyma and stroma, has the potential to be impaired by the dependence of the generation of ROS on the presence of oxygen. With the unorganized growth of vasculature, not all tumor tissue is properly vascularised leading to insufficient delivery of both oxygen and PS. Similar tumor types had different PS distributions, depending on vascularity, which resulted in an increased PDT effect in tumors with the most optimally distributed vascularity [26]. Impaired vascularity has proven to be an obstacle in direct PDT mediated tumor destruction.

\subsubsection{Vasculature effects}

The formation of new blood vessels, or neovascularization/angiogenesis, is a key process in cancer development [27]. The importance of adequate tumor vasculature becomes clear by the existence of necrotic and low oxygen regions inside tumors due to the unorganized formation of blood vessels. Damaging existing vasculature or inhibiting the formation of new vessels has detrimental consequences for tumor proliferation, and antiangiogenic therapeutics have been clinically approved for the treatment of cancers [28]. Damaging tumor vasculature has been shown to be an important factor in PDT efficacy. For example, much of the therapeutic effect of hematoporphyrin derivative (HPD) appears to be largely due to the consequences of disrupted blood flow [29]. Following PDT, endothelial and subendothelial cells are damaged. The direct damage to vasculature is significantly 
increased when the time between PS administration and light activation is shortened [30]. The influence of the interval between drug administration and light activation on vascular damage was investigated when the anti-tumor effect of verteporfin on rat chondrosarcomas was evaluated by varying the drug light interval (DLI). Intravascular PDT damages endothelial cells, causing them to round up, widening the interendothelial cell junctions and exposing the underlying tissue. Damaged endothelial cells may release clotting factors such as von Willebrand factor, activating platelets $[31,32]$. The activated platelets interact with the exposed subendothelium leading to platelet aggregation, thrombus formation and vessel occlusion [29,33]. Moreover, activated platelets induce vasoconstriction after PDT, further decreasing blood flow [34]. The impaired blood flow and blood vessel destruction, in time, will result in tissue hypoxia, nutrient deprivation and tumor destruction [29,30]. These features of PDT led some research groups to adopt the concept of vascular targeted PDT (VTP) to increase therapeutic efficacy. In studies comparing cellular targeted PDT with a vasculature targeted approach by modulating the DLI, efficacy increased when tumor vasculature was targeted with a short DLI $[31,35,36]$.

The enhanced therapeutic effect in these studies is probably due to longer lasting tissue hypoxia. When measuring the tissue oxygen levels during and after PDT and vasculature targeted PDT, $\mathrm{pO}_{2}$ drops significantly during both procedures due to the formation of ROS. However, in PDT tissue oxygen levels quickly recover, while after vasculature targeted PDT no such recovery is seen. Tissue reoxygenation after PDT lowers its therapeutic outcome, which can be avoided by the destruction of blood vessels ensuring long-lasting hypoxia and a better therapeutic outcome [37]. The vasculature disrupting effects of PDT are an important component of PDT efficacy.

\subsubsection{Immune reaction}

The third mechanism of PDT-induced tumor destruction is the initiation of an inflammatory response that is followed by host tumor immunity. PDT-induced oxidative stress can upregulate the expression of heat shock proteins (HSPs), transcription factors related to inflammation and release of inflammatory cytokines [38]. Tumor cell death is accompanied by the release of proteins and other molecules, called damage-associated molecular patterns (DAMPs), that can elicit a strong inflammatory response. Studies show that after PDT, HSPs such as HSP70 are either localized on the cell surface or in case of necrosis can be released extracellularly [39]. HSPs can bind tumor antigens and interact with 
Toll like receptors (TLRs), which is a major route of activating antigen presenting cells (APC) [40]. In addition, these interactions regulate the expression of inflammatory and immune response genes [41]. The origin of HSPs and other DAMPs can vary depending on the subcellular location of PDT action [42]. Other DAMPs observed after PDT are membrane breakdown products, such as lipid fragments and metabolites of arachidonic acid [43], ATP [44] or the ER protein Calreticulin [45]. Innate immune cells such as macrophages, lymphocytes and dendritic cells (DCs) are recruited by DAMPs during inflammation, to remove cellular debris and promote tissue healing [46]. Moreover, it has been suggested that adaptive immunity is also triggered leading to the systemic and long-term immunity after PDT [47].

\subsection{Photosensitizers}

PSs have typically been divided in generations based on the time of development and their specific characteristics. The first-generation PSs are the hematoporphyrins (Hp) that first arose in the $19^{\text {th }}$ century. The first $\mathrm{Hp}$, formed from dried blood, was a mixture of several porphyrins, each with their own characteristics [48]. It was initially used as a fluorescent diagnostic tool for cancers, but due to its heterotypical nature, large doses were needed to achieve desired effects. When processed further, a hematoporphyrin derivative was formed that had better tumor localization properties and could be used as a PS to treat gliomas by means of PDT. Another purification step resulted in the formation of Porfimer Sodium (Photofrin), which was approved by the FDA and EMA to be used in the clinic to treat cancers [49-51]. Even though it is the most widely used PS for the treatment of cancers, Photofrin still is a complex mixture of molecules with relatively poor tissue selectivity, low absorption of light and poor tissue penetration of light. High Photofrin dosage is needed for therapeutic effect leading to prolonged patient skin sensitivity after PDT [52]. This led to the development of second-generation PSs that were made to overcome the limitations of the first generation. They consist of all sorts of porphyrins generally divided into porphyrins, chlorins, pheophorbides, bacteriopheophorbides, texaphyrins and phthalocyanines, each group consisting of numerous types of PSs (reviewed in [53]). Second-generation PSs aim to increase PS purity and reproducibility to have better control over production and drug behaviour. The goal of using these second-generation PSs was to achieve better tumor selectivity and reduce the overall drug dose. The added effect of lower doses means the product is cleared faster and skin photosensitivity which is one of the main limitations of 
PDT in the clinic, can be reduced from weeks to days. The photochemical properties of these new PS were adjusted, so as to utilise the preferential absorption of light at longer wavelengths, so they can be used to treat tumors in deeper tissues or utilize fewer implanted light sources [52]. A third generation PS refers to modified second-generation PSs using nanocarriers or biologic conjugates, such as antibodies to improve their physical, chemical and therapeutic properties. This will also ensure lower dosage and fewer unwanted side effects.

\subsubsection{An ideal PS}

The definition of an ideal PS is often described based on preferential characteristics. Several properties are generally accepted as ideal $[51,54]$. The PS should have low dark toxicity and preferably no toxicity at administration (no allergic reactions or hyposensitivity). Moreover, administration should be easy and feasible via different routes without any pain. It should have a high absorption band, preferably in the near infrared (NIR), for optimal tissue penetration, yet with enough energy to generate singlet oxygen. It should have a high yield of ROS during illumination. High tumor selectivity and rapid clearance from the body will minimize photosensitivity of the skin. Moreover, the PS should be pure and easily produced, as well as be stable enough for long storage. The search for new and improved PSs is an active field of research as can be seen by the currently ongoing clinical trials looking to assess safety and efficacy of newer PSs and the many preclinical reports of completely novel PSs. One of the focus points is water solubility to improve PS circulation and efficacy in aqueous surroundings. By rationally designing molecules it is possible to synthesize watersoluble PSs that also accumulate at desired locations [55]. Adding functional groups to the PS allow bio-conjugation of moieties capable of accentuating desirable properties [56].

\section{What affects PDT efficacy}

\subsection{Light}

The therapeutic efficacy of PDT depends on the properties of the light used to activate the PS. In a superficial approach it has to both penetrate skin and tissue to reach the target site and be able to activate the PS in situ. In an intraluminal or interstitial setting, the placement of multiple light sources is an important consideration. The penetration of light in tissue is a complex process, which is dependent on the optical properties of the tissue at the wavelength of light used. There is significant heterogeneity between tissues and even within 
tissues, with numerous molecules influencing light scattering and absorption. At shorter visible wavelengths, efficacy can be limited due to the absorption by endogenous chromophores such as haemoglobin, whereas at longer wavelengths water can absorb light. This limits the range of wavelengths to optimally penetrate tissue between $600 \mathrm{~nm}$ and 1300 $\mathrm{nm}$. However, light with a wavelength longer than $850 \mathrm{~nm}$ does not provide sufficient energy needed to activate the PS to its triplet state and to generate singlet oxygen. As such, the "therapeutic window" for the majority of PDT applications lies in the red region of the spectrum between 620 and $850 \mathrm{~nm}$ achieving optimal tissue penetration and PS activation [57].

For the delivery of light, both lasers and incandescent light have proven to be effective [58]. The source of the light should be fitted to the PS photophysical characteristics (absorption spectrum), type of disease (location, size of tumor, tissue type) and usability (cost, size, handling). With topical lesions, at for instance the skin, it is easier to use lamps instead of lasers since they are cheaper to maintain, user friendly and their broad emission can be used with several PSs. Lasers are widely used in clinical PDT as they are powerful, can be coupled to optical fibres that can be used to interstitially illuminate deeper located tumors with the application of diffusing tips. Numerous studies are looking to optimize light sources with new approaches. For instance, the use of Light Emitting Diodes (LED) in PDT is investigated [59-61]. LEDs are cheap, easy to manufacture, have a high-power output and can be used for a broad range of wavelengths. They are however of limited use for large tumors where an interstitial approach is required.

\subsection{Oxygen}

The availability of sufficient tissue oxygen is crucial in the efficacy of cancer therapy. The presence of hypoxic areas in tumors proves to be a major obstacle in the treatment of solid tumors [62]. One indirect reason is that hypoxia is usually induced by impaired tumor vasculature, meaning drug delivery routes are impaired. Another reason is the importance of oxygen for the therapeutic effect of, for instance, radiotherapy and certain chemotherapies $[62,63]$. In PDT, the formation of singlet oxygen needs ground state oxygen, therefore tissue oxygenation heavily influences PDT efficacy $[64,65]$. Hypoxic areas inside tumors have proven obstacles for PDT efficacy and tumors with hypoxic areas are considered PDT resistant [66]. Indeed, when the main vasculature of a tumor is occluded, the effect of PDT is considerably ablated [67]. Increasing tumor oxygenation by hyperbaric oxygen 
therapy has been shown to improve tumor response to chemotherapy and radiotherapy [68]. In PDT studies there are conflicting reports. When Photofrin is used in combination with hyperoxygenation by letting tumor bearing mice breathe under pressurized conditions, improved cell killing after PDT is observed [69,70]. Other PSs show the same oxygen dependent efficacy [71] while yet others seem to be unaffected by lower tissue oxygen levels [72].

\subsection{PS uptake and localization}

With the limited action radius of ROS and especially singlet oxygen, the precise localization of the PS is crucial for its therapeutic effect. Understanding and controlling PS localization greatly increases the potential of PDT. From the moment of administration, until the PS has reached the target location, various physical, chemical and biological events take place that together influence the end location of the PS. For example, when intravenously administered, the PS will first encounter serum proteins to which it will bind. Different PS will associate differently to these proteins and therefore the pharmacokinetics and distribution will vary accordingly [19]. The PS has to extravasate the blood vessels to reach the tumor site, thereafter associating with the extracellular matrix or the cells within the tumor. As mentioned earlier, PSs have been found to localize in numerous organelles which is dependent on the structural characteristics of the PS. It has been shown that overall charge, lipophilicity and overall structure predominantly determine cellular uptake and subcellular localization of a PS and ultimately determine its therapeutic effect [73].

\section{PDT in clinical trials}

\subsection{Clinically approved PS}

In the clinic, PDT can be used in conjunction with surgery, radiotherapy or chemotherapy, due to its mode of action. Because it is activated locally and has limited tissue penetration, PDT is relatively tissue sparing with, in some cases, good cosmetic outcomes. This makes it especially suitable for skin conditions and sensitive areas such as the head and neck [74]. Moreover, it lacks the adverse events seen in radiotherapy and systemic chemotherapy. Unfortunately, intravenously administered PSs induce prolonged periods of skin photosensitivity, during which patients need to avoid light [74]. 
Despite PDT having several favourable characteristics to standard treatment

modalities, only limited number of PSs have received regulatory approval for the treatment of cancers by the FDA and EMA. The list of approved PSs is summarized in Table 1. Photofrin was the first PS to get clinical approval for the treatment of several cancers. However, this PS is a complex mixture of molecules with relatively poor tissue selectivity, low absorption of light and, at the wavelength needed for its activation, light has poor tissue penetration. A high dosage of Photofrin is needed to achieve the desired therapeutic effect, leading to long circulation times and prolonged patient photosensitivity [75]. The second PS to receive approval is the second-generation Temoporfin (Foscan), which has been approved by the EMA for the treatment of advanced head and neck squamous cell carcinomas. It absorbs light at longer wavelengths and has shorter circulation time, improving its safety profile compared to first-generation Photofrin.

For vascular targeted PDT, the first PS Palladium-metalated bacteriopheophorbide, known as TOOKAD ${ }^{\circledR}$ (Negma Lerads/Steba Biotech), has been approved in Europe and Israel for the treatment of men with low-risk prostate cancer in 2017 [75]. This treatment can cause fast vascular occlusion and subsequently cancer cell death, within a $5 \mathrm{~mm}$ range of the optic fibres. Currently, a phase III clinical trial is ongoing in the US, in which TOOKAD ${ }^{\circledR}$ is applied for the treatment of intermediate risk prostate cancer (NCT04225299). The watersoluble derivative of $\mathrm{TOOKAD}^{\circledR}(\mathrm{WST} 11)$, is evaluated in phase I clinical trials for the treatment of esophagogastric cancer (NCT03133650) and upper tract urothelial carcinoma (NCT03617003). Despite the ongoing clinical trials, FDA did not approve the application of WST11 for the treatment of localized prostate cancer after evaluation of the phase III clinical trial results (NCT01310894). 
Table 1. Overview of clinically approved PSs.

\begin{tabular}{|c|c|c|c|}
\hline PS & $\begin{array}{c}\text { Excitation } \\
\text { wavelength }\end{array}$ & Approved & Indication \\
\hline $\begin{array}{l}\text { Porfimer sodium / } \\
\text { Photofrin }^{\circledR}\end{array}$ & $630 \mathrm{~nm}$ & $\begin{array}{l}\text { Worldwide, } \\
\text { withdrawn in EU } \\
\text { for commercial } \\
\text { reasons }\end{array}$ & $\begin{array}{l}\text { High grade dysplasia in } \\
\text { Barret's Esophagous. } \\
\text { Obstructive esophageal or } \\
\text { lung cancer. }\end{array}$ \\
\hline $\begin{array}{l}\text { 5-ALA / Ameluz / } \\
\text { Levulan }^{\circledR}\end{array}$ & $635 \mathrm{~nm}$ & Worldwide & $\begin{array}{l}\text { Mild to moderate actinic } \\
\text { keratosis/ Image guided } \\
\text { surgery in brain tumors }\end{array}$ \\
\hline $\begin{array}{l}\text { Metvix / } \\
\text { Metvixia }^{\circledR}\end{array}$ & $\begin{array}{c}570-670 \\
\mathrm{~nm}\end{array}$ & Worldwide & $\begin{array}{c}\text { Non-hyperkeratotic actinic } \\
\text { keratosis and basal cell } \\
\text { carcinoma }\end{array}$ \\
\hline $\begin{array}{c}\text { Temoporfin / } \\
\text { mTHPC / Foscan }{ }^{\circledR}\end{array}$ & $652 \mathrm{~nm}$ & Europe & $\begin{array}{c}\text { Advanced Head and neck } \\
\text { cancer }\end{array}$ \\
\hline $\begin{array}{l}\text { Talaporfin / NPe6 } \\
\text { / Laserphyrin }^{\circledR}\end{array}$ & $664 \mathrm{~nm}$ & Japan & $\begin{array}{l}\text { Early centrally located lung } \\
\text { cancer }\end{array}$ \\
\hline $\begin{array}{l}\text { Verteporfin / } \\
\text { Visudyne }^{\circledR}\end{array}$ & $690 \mathrm{~nm}$ & Worldwide & $\begin{array}{l}\text { Age-related macular } \\
\text { degeneration }\end{array}$ \\
\hline $\begin{array}{l}\text { Synthetic } \\
\text { hypericin / } \\
\text { SGX301 }\end{array}$ & $\begin{array}{l}570-650 \\
\mathrm{~nm}\end{array}$ & $\begin{array}{l}\text { Orphan status in } \\
\text { EU }\end{array}$ & Cutaneous T-cell lymphoma \\
\hline $\begin{array}{l}\text { Redaporfin / } \\
\text { LUZ11 }\end{array}$ & $749 \mathrm{~nm}$ & $\begin{array}{l}\text { Orphan status in } \\
\text { EU }\end{array}$ & Biliary tract cancer \\
\hline TOOKAD $^{\circledR}$ & $652 \mathrm{~nm}$ & Israel/Europe & Low-risk prostate cancer \\
\hline
\end{tabular}




\subsection{Organ specific PDT in clinical trials}

Even though PDT has been investigated for decades, only few PSs are approved for use in a clinical setting, as described in 4.1. However, recognizing the potential of PDT, investigators are trying to evaluate the safety, feasibility and efficacy of a variety of PSs for PDT for numerous types of cancer and increase utilization of PDT in the clinic. Most trials are trying to establish the optimal dosage of PS or administered light for compounds clinically approved for different types of cancer. In this section trials and some of the studies done during the last ten years that investigate the use of PDT for lung, head and neck, pancreas and brain cancers are described.

\subsubsection{Lung}

In case of inoperable disease and failure or refusal of other treatments, PDT has potential as a palliative standalone or combination therapy due to lack of systemic effects and its organ-function sparing action. Additionally, as opposed to radiotherapy, the working mechanism of PDT allows repeated treatments. PDT was deemed well-tolerated and effective as part of a multi-modal treatment for endobronchial non-small cell lung cancer (NSCLC) in a small retrospective study [76]. The palliative efficacy and safety of PDT as part of a multi-modal treatment was evaluated in a single centre prospective pilot study with patients suffering from advanced NSCLC with central airway obstruction [77]. PDT consisted of an intravenously administered water-soluble chlorin E6 complex (Radachlorin ${ }^{\circledR}$ ) followed by endoluminal irradiation via fibroptic bronchoscopy. All patients showed improvement of their symptoms with significantly improved lung capacity and function. One year post-PDT survival was improved significantly for PDT treated patients compared to the one-year survival rate mentioned for patients with NSCLC treated with systemic chemotherapy alone $[77,78]$. Talaporfin-PDT, which received approval as a lung cancer treatment in Japan, was combined with chemo-radiation therapy, radiotherapy or chemotherapy to palliatively treat intractable lung cancer with airway stenosis. This multimodal approach significantly relieved airway obstruction, improved lung capacity parameters and quality of life, ultimately prolonging patient survival [79].

PDT could also be a valuable addition to the adjuvant therapy regimens. A study showed patients receiving postoperative Photofrin-PDT have an improved mean survival time when compared to patients treated with standard postoperative care [80]. In a different study, patients undergoing radical pleurectomy followed by post-operative PDT showed 
unusually long survival, despite recurrences and no increased progression-free survival, most likely due to the preservation of the lung and/or the PDT effect [81]. These studies indicate PDT can be easily implemented in standard care regimens to improve therapy outcome.

As a stand-alone treatment, Photofrin-PDT proved a good alternative for palliative chemotherapy or radiotherapy in unresectable lung cancer as it achieved an overall response of nearly $87 \%$ and improved patient quality of life. It even showed curative potential with several cases of complete remission [82]. However, photosensitivity, secretions and pain were common adverse events. Another major drawback of Photofrin-PDT was the fact it becomes less effective with tumors over $1 \mathrm{~cm}$ in diameter $[83,84]$. As such, the guidelines of the American College of Chest Physicians in 2003 recommended that PDT is only suitable for lesions under $1 \mathrm{~cm}$ in diameter based on results with Photofrin. However, secondgeneration PSs with deeper tissue penetration prove more effective with larger lesions. No significant difference in efficacy was observed between tumors under or over $1 \mathrm{~cm}$ when using Talaporfin [85]. The same group showed that Talaporfin-PDT was also effective in treating patients with multiple primary lung cancer (MPLC). All MPLC patients that received PDT, either alone or in combination with surgery, achieved complete remission indicating PDT can be used for multiple lesions under certain conditions [86].

Other newer PSs such as 2-[1-hexyloxyethyl]-2-devinyl pyropheophorbide-a (HPPH) are also finding their way to clinical trials. HPPH is a chlorin based PS which absorbs light at $665 \mathrm{~nm}$ and has a lower risk of skin sensitivity due to its shorter half-life compared to Photofrin [87]. A Phase I dose escalation study showed HPPH-PDT is capable of achieving high rates of complete remission that is retained for months in patients with carcinoma in situ and micro invasive cancer of the central airways [88]. Minor photosensitivity was reported but overall adverse events were limited. Two ongoing clinical trials are investigating two new PSs for their safety and efficacy in lung cancer. One trial is investigating the water-soluble palladium-bacteriochlorophyll (WST11) in obstructive NSCLC (EudraCT ID: 2009-011895-31). The other study is an open-label Phase IIb study to evaluate the safety, tolerability and efficacy of Fotolon (Chlorin e6-PVP) for the treatment of obstructing NSCLC (EudraCT ID: 2013-001876-39).

\subsubsection{Head and Neck}

Initially PDT was explored for treating early stage, easy to reach, head and neck cancer and focussed on superficially growing basal and squamous cell carcinoma (SCC) 
using HPD [89-91]. Photofrin has been shown to be effective in the treatment of oral squamous cell carcinoma (OSCC) and dysplasia but post-PDT photosensitivity and limited tissue penetration confirm the preference for second-generation PSs [92]. The use of secondgeneration PSs, such as 5-aminolevulinic acid and mTHPC (Foscan) for early stage of head and neck cancer results in good clinical and cosmetic outcome without the adverse events of the prolonged skin-photosensitivity seen after HPD and Photofrin II $[93,94]$. With HPPH, a different second-generation photosensitizer, PDT has also been used for high risk dysplasias, carcinoma in situ and SCC and showed promising complete remission rates and mild adverse events [95]. Recently a Phase Ib study showed that HPPH-PDT can be safely used to treat early stage larynx cancer with $85 \%$ complete remission at the maximum tolerated dose [96]. Secondary to that, the use of mTHPC with its far-red absorption combined with the possibility to illuminate interstitially meant that also thicker lesions could be treated $[97,98]$.

Several studies were performed investigating mTHPC-PDT for the treatment of OSCC and oropharynx SCC. Complete response rates of $96 \%$ and $86 \%$ have been reported for carcinoma of the lip and oral/oropharynx SCC after a follow-up of 12 months and 37 months respectively $[94,99]$. Both studies report good functional and cosmetic outcome. Two studies have compared surgery with mTHPC-PDT for OSCC on the tumor response, disease free survival and local or overall survival. Recurrent and persistent nasopharyngeal carcinoma (NPC) and advanced head and neck cancers are a serious problem as conventional treatment options are often exhausted. Treatment of nasopharyngeal carcinoma with mTHPC-PDT can result in complete remission and lead to prolonged survival for patients [100]. A prospective study using ultrasound guided interstitial PDT for deep seated pathologies showed good results with most patients reporting improved quality of life and improvement of limb function [101]. In another study, interstitial PDT in a group of 45 patients with persistent or recurrent head and neck cancer prolonged survival of the 33 patients $(73 \%)$ who responded to the treatment to a median of 16 months [98].

mTHPC is approved in Europe for the palliative treatment of advanced head and neck cancers, often when surgery or radiotherapy are no longer viable options. It proved effective in inducing tumor reduction and complete remission was achieved in half of the patients with head and neck cancer that had exhausted all other curative treatment options. Moreover, PDT stabilized disease leading to a significantly prolonged survival in patients that responded to treatment compared to non-responders confirming mTHPC-PDT can be 
considered as a valuable treatment option [102]. A recent report describes a small but impressive study investigating the use of MTHPC-PDT for the treatment of recurrent SCC of the base of tongue after salvage surgery following (chemo-) radiation failure. The two patients treated were disease free after 24 and 42 months follow-up [103]. mTHPC-PDT has also been shown to be a safe adjuvant therapy for recurrent malignant tumors of the paranasal sinuses and recurrent sino-nasal skull base tumors [104]. Currently, a clinical trial is ongoing with Redaporfin (LUZ11), which investigates the tolerability, pharmacokinetics and antitumor effect in a dose escalation study in patients with advanced head and neck cancer (EudraCT ID: 2013-003133-14).

\subsubsection{Pancreas}

Owing to its mode of action, PDT circumvents most tumor therapy resistance mechanisms and therefore is a modality with potential in effectively treating pancreatic cancer. The first clinical trial of PDT in the treatment of locally advanced pancreatic cancer was conducted in 2002 using mTHPC [105]. PDT could produce tumor necrosis in all patients with low morbidity and mortality. The results of a Phase I trial suggested applying PDT for localized cancers in patients who are not surgical candidates. Recently, a Phase I/II dose escalation study was completed in which the safety and efficacy of verteporfin were evaluated in patients with locally advanced pancreatic carcinoma (EudraCT ID: 2006004097-28). However, the results have not been published yet.

\subsubsection{Brain}

The tissue sparing characteristic of PDT could offer an alternative to conventional methods for aggressive and difficult to treat tumors such as glioblastoma multiforme. Alternatively or in combination, the fluorescence of the PS has been used to guide the resection of tumors, known as fluorescence guided resection. Here, 5-aminolevulinic acid (5-ALA), which converts into the active fluorescent molecule protoporphyrin IX (PpIX), has been mostly used. PpIX is excited by light of $357-440 \mathrm{~nm}$ wavelength, which although not appropriate for deep tissue imaging, is sufficient for superficial detection. Stummer and colleagues reported a more complete resection of tumors that led to improved progressionfree survival in patients with malignant glioma [106]. A similar observation was documented in a study where 5-ALA fluorescence guided resection was followed by interstitial PDT [107]. Here the cumulative 6 months progression free survival rates were $41 \%$ and $21 \%$, for fluorescence guided resection combined with PDT, compared to PDT alone. 
In a different study, the combination of 5-ALA and Photofrin was employed for

fluorescence guided resection, followed by repetitive PDT [108]. This controlled trial showed a survival advantage (52.8 weeks of mean survival in the study group of 13 patients, compared to 24.6 weeks in the control group of 14 patients) without added risks to the patients with glioblastoma multiforme. Muller and colleagues reported on the results of Photofrin PDT alone and despite the encouraging results, the authors suggest that higher light doses than the ones employed in this study may be required for better responses [109].

A preliminary clinical study tested the safety and efficacy of Talaporfin-PDT for patients with completely removed, subtotally removed or partially removed gliomas, which were either recurrent or newly diagnosed. PDT had positive results in newly diagnosed tumors with high rates of tumor response and prolonged patient survival. With recurrent gliomas, PDT performed considerably worse with low tumor response, possibly due to inadequate penetration of tissue at the light-dose regimen applied in this study [110]. A follow-up study further examined the efficacy and safety of intraoperative Talaporfin for recurrent or newly diagnosed glioblastoma multiforme [111]. PDT considerably increased both overall and local median progression free survival and overall survival was prolonged compared to these parameters after chemo-radiation therapy or fluorescence guided resection as reported in literature [106,112]. Again, results were better for newly diagnosed gliomas [111]. Recently, two clinical trials were conducted. One is a Phase I study assessing PDT for recurrent malignant brain tumors with poor prognosis using Photofrin. Here, overall survival for 3 years post PDT treatment was followed (NCT01682746). The other is a phase II clinical trial investigating PDT with Photofrin for recurrent high-grade gliomas in adult patients (NCT01966809). These clinical studies show PDT can be a beneficial addition to glioma treatment modalities, especially for newly diagnosed tumors.

\subsection{Current limitations of PDT in the clinic}

PDT proves to be effective in inducing tumor responses as well as improving patient survival and quality of life. Efficacy is seen when PDT is part of a multimodal approach or used as a first-line treatment for premalignant or early disease and as standalone palliative treatment. Even though PDT shows great potential, there are still some limitations that prevent a firm position for PDT in standard care management of cancer. When reviewing the clinical trials and studies done over the last ten years, some general problems become evident. A major problem is related to the adverse events associated with PDT. With 
systemically administered PSs, especially of the first generation, skin photosensitivity is one of the most common adverse events. Patients have to avoid sunlight and strong artificial light for weeks, which is highly undesirable when they are nearing the end of life. Another adverse event often reported is pain. The main mechanism in PDT induced pain has yet to be elucidated, but several studies have found some predictors of pain. The biggest predictors appear to be the size of the treated area while location, PS type, lesion type, gender, age and light protocol have also been mentioned [113]. Several strategies of pain management have been tested, but none fully relieved PDT induced pain [113]. The occurrence of adverse events, such as inflammation, fever and nausea are typically location dependent but are often successfully managed with medication. Another drawback is the decreasing efficacy of PDT for larger lesions, especially with first-generation PSs. Due to inadequate tissue penetration of light or PS, bulky or deep seated tumors are difficult to treat with PDT. Especially evident with Photofrin and 5-ALA, the limited penetration of the appropriate light prevents sufficient depth of tumoricidal action [114]. Even second-generation PSs perform less in larger lesions in which case surgery is more effective $[115,116]$. The most effective PSs tend to be hard to dissolve due to hydrophobicity and can form aggregates that have trouble penetrating tumor tissue [19]. Finally, due to the longer retention of hydrophobic PSs in the body and accumulation in healthy tissues, damage to the surrounding normal tissues, such as nerves and muscles often occurs upon illumination. Depending on the cancer type and tissue affected, such damage can lead to considerable reduction in quality of life.

\section{Strategies to overcome current limitations and improve therapeutic efficacy}

Several strategies can be applied to overcome limitations mentioned above. Using more hydrophilic PSs can increase clearance from the body, reducing side effects such as photosensitivity and damage to the normal tissues. Therefore, design of new PSs or modification of known PSs is an active field of research [117]. To improve PDT tissue penetration in bulky or deep seated tumors, interstitial PDT (iPDT) can be applied using optical fibers to guide light deeper into the tissues, thereby improving the depth of PDT action [118]. Even though iPDT is already applied in the clinic, optimal fiber placement and light dosimetry remain topics of research. Over the years, many efforts have been made in the field of PDT to target PS specifically to the site of the tumor, also necessary for water soluble PS that alone do not associate with cells. Targeting of certain receptors which are only present or more abundant on tumor cells or tumor vasculature allows for the usage of 
different types of targeting moieties such as antibodies, and antibody fragments such as nanobodies to deliver the PS specifically to the tumor site. Alternatively, nanotechnologybased PS delivery systems such as polymeric nanoparticles and liposomes, can improve pharmacokinetics of hydrophobic PSs, leading to less damage to surrounding tissues [117].

Besides trying to overcome the currently existing limitations of PDT in the clinic, research is focussed on improving certain aspects to improve PDT efficacy. PDT can be used either pre, intra or post-operatively and in combination with other modalities to improve treatment outcome. Due to its mode of action it can easily be used in combination with chemotherapy, radiotherapy and surgery. Alternatively, a combined approach of conventional PDT that targets both cancer cells and tumor vasculature is one of the approaches that can help to improve therapeutic effects of PDT.

\subsection{Combination of cancer and vascular targeted PDT}

As described under 2.3.2, the damage induced to tumor vasculature upon PDT is inversely correlated with the duration of the time interval between PS administration and illumination. Shorter drug light intervals have been explored for vascular targeted PDT, while longer intervals for the conventional cancer targeted PDT.

In preclinical studies, Dolmans and co-workers investigated simultaneous targeting of vasculature and cancer cells in an orthotopic breast cancer model by fractionated PS dosing PDT before single light administration ( $4 \mathrm{~h}$ and $15 \mathrm{~min}$ after PS MV6401 administration). The fractionated drug dose PDT more effectively induced tumor growth delay than the same total dose given as a single dose, either at $4 \mathrm{~h}$ or at $15 \mathrm{~min}$ before light administration. The long-term effect of the fractionated drug PDT on blood flow was also more extensive than single-dose PDT [119]. Similarly, combination of a long interval (cellular targeting, $3 \mathrm{~h}$ after PS injection) PDT and a short interval (vascular targeting, 15 min after PS injection) PDT was explored by Chen et al. in a prostate cancer model [36]. Fluorescence microscopic studies indicated that tumor localization of verteporfin changed from predominantly within the tumor vasculature at $15 \mathrm{~min}$ after injection, to being throughout the tumor parenchyma at $3 \mathrm{~h}$ after injection. Light treatment at $15 \mathrm{~min}$ after verteporfin injection induced significant tumor vascular damage, as manifested by tumor blood flow reduction and increase in the tumor hypoxic fraction. In contrast, the vascular effect observed after the same light dose delivered $3 \mathrm{~h}$ after administration of verteporfin was an initial acute decrease in blood flow, followed by recovery to the level of control. The 
combination of $3 \mathrm{~h}$ interval PDT and 15 min interval PDT were more effective in inhibiting tumor growth than each individual PDT treatment. However, it was found that the combined treatment with the sequence of $3 \mathrm{~h}$ interval PDT before $15 \mathrm{~min}$ interval PDT led to a superior

antitumor effect than the other combined PDT treatments. Histologic studies confirmed that this combined treatment led to damage to both tumor vasculature and tumor cells.

Both studies suggest that targeting both tumor cellular and vascular compartments can be an effective and safe way to enhance PDT damage to tumor tissue.

\subsection{Improving PS distribution and specificity}

By improving PS localization, it can be expected that there will be increased efficacy in tumor destruction and fewer side effects related to off-target localization [120]. Additionally, more effective targeting would also decrease the PS dosage needed, further limiting side effects. Targeting can be achieved on tissue, cellular and sub cellular level in PDT. Selectively addressing tumor tissue is particularly important in reducing side effects. As of now, (partial) tumor selectivity is mostly dependent on the passive EPR effect and the localized PS activation by specific tissue illumination. Actively targeting tumor cells can be achieved by a variety of approaches, among which conjugating to monoclonal antibodies or nanobodies.
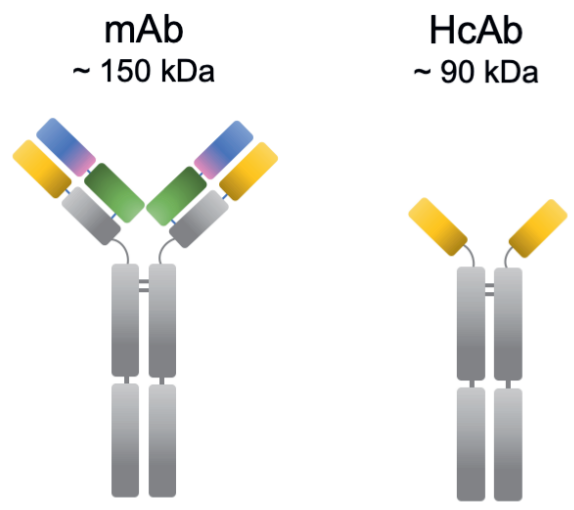

nanobody

$\sim 15 \mathrm{kDa}$

Figure 2. Schematic representation of conventional monoclonal antibody, heavy chain only antibody and nanobody. Nanobodies are the variable domains of heavy chain only antibodies. Compared to the antibody $(\sim 150 \mathrm{kDa})$, the molecular weight of nanobody is ten times smaller $(\sim 15 \mathrm{kDa})$. 


\subsubsection{Antibodies}

Conjugation of PSs to monoclonal antibodies $(\mathrm{mAb})$ has been employed to enhance tumor specificity. This approach, referred to as photoimmunotherapy, was first mentioned in 1983 by Mew and co-workers who conjugated HPD with a mAb to a tumor specific antigen on myosarcoma cells [121]. This increased HPD specificity and directed tumor cell killing both in vitro and in vivo. Results showed that the mAb conjugated HPD specifically targeted the antigen presenting tumor cells in vivo and significantly increased tumor inhibition after illumination. By increasing the DLI, the specific targeting effect was even more pronounced as the mAb accumulated in the tumor over time [121]. Since then, several mAbs have been approved for clinical use in cancer therapies, which could also be used for photoimmunotherapy (PIT) [122]. Targeting EGFR proves successful as several studies have investigated the use of anti-EGFR mAbs for PS targeting [123-125]. In a proof of concept study, verteporfin was coupled to an anti-EGFR mAb. Antibody conjugation increased verteporfin uptake by EGFR expressing A431 cells compared to untargeted verteporfin. In vivo, increased concentrations of anti-EGFR-verteporfin were detected in EGFR positive tumors compared to EGFR negative tumors or healthy tissue. Moreover, tumor inhibition and mouse survival after irradiation was increased with targeted verteporfin compared to untargeted verteporfin or controls, indicating $\mathrm{mAb}$ targeting could both increase selectivity and therapeutic effect [123].

The phthalocyanine derivative PS IRDye700DX is relatively hydrophilic PS, compared to the other PSs, which has been used extensively in preclinical studies for therapeutic applications [124,126-128]. This PS absorbs light at a longer wavelength (689 $\mathrm{nm}$ ) than most PSs, such as Photofrin or Foscan, increasing depth of light penetration. The commercially available mAbs Trastuzumab and Panitumumab, which are directed against HER2 and EGFR respectively, were used to provide selectivity to the water-soluble PS IRDye700DX [124]. In vitro studies showed that the mAb-IRDye700DX conjugates only induced death in cells expressing their respective receptors. Moreover, the conjugates needed to be bound to the cell to have photocytotoxic effect, as IRDye700DX alone would not associate with cells and unbound conjugates did not induce cell death. In vivo studies using xenografted mice bearing both receptor-positive and -negative tumors showed receptor dependent IRDye700DX accumulation and tumor eradication [124]. Currently, a Phase III trial is ongoing, where the mAb Cetuximab targeting EGFR is conjugated to the PS 
IRDye700DX for PDT of patients with recurrent head and neck cancers (NCT02422979). The outcome of this trial will certainly influence further evaluation of these or other targeted PDT approaches in the clinic.

A known restraint of antibody-targeted therapy is the insufficient intra-tumoral distribution of the therapeutics [129]. This occurs especially when an antibody has a high binding affinity for its receptor and/or the tumor cells express high levels of target antigen. In this case antibodies are saturated in the immediate perivascular space and cannot penetrate deeper into the tumor, a phenomenon known as the "binding site barrier". More uniform distribution of antibodies can be achieved by adding a second antibody with either lower affinity or lower antigen expression which enables this second antibody to be more bioavailable deeper in the tumor. In addition, intratumoral heterogeneity is one of the leading causes of therapeutic resistance and treatment failure and one of the main reasons for poor overall survival in cancer patients. Single receptor targeted therapies generally eradicate one subpopulation while the other surviving subpopulations of tumor cells will proliferate and regrow, promoting tumor progression. Therefore, combined approaches can potentiate the therapeutic response. Addressing these two concerns a mixture of differentially targeted PS conjugations was tested. IRDye700DX was conjugated to either anti-EGFR (Panitumumab) or anti-interleukin-2 receptor-alpha (Basiliximab). In vivo studies showed a better intratumoral distribution of the PS and a beneficial anti-tumor effect with improved survival when combining Pan-IRDye700DX and Bas-IRDye700DX conjugates compared to either conjugate alone [125].

\subsubsection{Nanobodies}

Despite antibodies' therapeutic potential, conjugating PSs to relatively large mAbs increases the PS half-life, which is an undesired characteristic leading to prolonged photosensitivity [130]. As such, it was attempted to use smaller antibody fragments [131135]. More recently, nanobodies (NBs) have been evaluated as an alternative for mAbs. NBs only consist of the variable binding domain of heavy chain antibodies that, despite their size, can specifically bind antigens such as EGFR. Oliveira et al. directly compared anti-EGFR NBs with mAbs (cetuximab) as targeting probes for a near-infrared imaging of tumors in vivo. The NB conjugates showed faster tumor accumulation and better intra-tumoral distribution compared to cetuximab [136]. The same nanobody was evaluated for targeted PDT, directly conjugated to IRDye700DX (PS) [137]. Incubating cells with no, or different 
expression of EGFR with the NB-PS conjugates showed a correlation between EGFR expression and NB binding, based on PS fluorescence. No binding to cells was observed of free PS or R2-PS (R2 being a negative control nanobody). Subsequently, cell death was only observed in cells with high EGFR expression, mediated by NB targeting EGFR conjugated to the PS. The first in vivo proof of principle study was conducted with these NBs conjugated to PS, where light was applied $1 \mathrm{~h}$ post administration of the conjugates, in a model of oral squamous cell carcinoma developed in the tongue of mice [126]. This approach led to extensive tumor damage, i.e. (80-90\% tumor necrosis with minimal toxicity to the surrounding tissues, thereby highlighting the selectivity of this approach. In the same study, cetuximab-PS led to $25-70 \%$ tumor necrosis. Viable tumor areas were clearly left after PIT, which was not observed with nanobody-targeted PDT. These observations are in line with the difficult tumor penetration of large antibodies, compared to a more homogenous distribution for nanobodies. Further evaluation of the nanobody-targeted PDT will reveal the full potential of this promising approach. 


\section{Aims and outline of the thesis}

Nanobody targeted PDT has recently been described as a promising approach, using EGFR targeted nanobodies, allowing illumination of the tumor area shortly after administration of the conjugates and, upon illumination, leading to specific tumor damage. The first aim of this thesis was to explore nanobody-targeted PDT with molecular targets other than EGFR; the second aim was to understand the vascular effects originated from nanobody-targeted PDT; and the third aim was to explore the combination of nanobody targeting cancer and endothelial cells for more effective PDT.

In Chapter 2 we investigated the potential of G protein-coupled receptors (GPCRs) as targets for nanobody-targeted PDT, using the HCMV-encoded chemokine receptor US28 as a proof of concept, to eradicate US28 expressing glioblastoma cells in 2D and 3D cell models. In Chapter 3 the ability of anti c-Met targeting nanobody to kill Met-expressing cells by PDT was explored. In Chapter 4 we investigated the tissue biodistribution of monovalent and biparatopic EGFR-targeted nanobody-PS conjugates. Cellular response and in particular vascular effects of EGFR-targeted PDT were evaluated in vivo using intravital microscopy, and DCE-MRI, complemented with ex vivo analysis of tumor tissues. With the intention to explore vascular targeted PDT (VTP) at a molecular level in more detail, studies performed in the last 15 years on the molecular targeting of PS to the tumor vasculature were reviewed in Chapter 5. The review provides an overview of the targeting moieties which have been explored to deliver PS specifically to the tumor vasculature in order to reduce side effects and increase treatment efficacy. In Chapter 6 we describe the development of nanobodies targeting VEGFR2 for endothelial cell targeted PDT. Higher expression of this tyrosine kinase receptor on tumor vasculature and lower expression in normal vessels makes it an ideal target for selective delivery of therapeutics to the tumor vasculature. Our hypothesis is that dual targeting of endothelial and cancer cells will likely potentiate nanobody-targeted PDT and improve therapeutic efficacy. Chapter 7 summarizes the obtained results and presents future perspectives on nanobody-targeted photodynamic therapy. 


\section{References}

1. Calixto, G.M.F.; Bernegossi, J.; de Freitas, L.M.; Fontana, C.R.; Chorilli, M.; Grumezescu,

A.M. Nanotechnology-based drug delivery systems for photodynamic therapy of cancer: A review. Molecules 2016, 21, 342-360,

2. Felsher, D.W. Cancer revoked: Oncogenes as therapeutic targets. Nature Reviews Cancer 2003, 3,375-380.

3. Bacellar, I.O.L.; Tsubone, T.M.; Pavani, C.; Baptista, M.S. Photodynamic efficiency: From molecular photochemistry to cell death. International Journal of Molecular Sciences 2015, 16, 20523-20559.

4. Henderson, B.W.; Dougherty, T.J. How does photodynamic therapy work? Photochemistry and Photobiology 1992, 55, 145-147.

5. Wagnieres', G.A.; Star2, W.M.; Wilson, B.C. Invited Review ln Vivo Fluorescence Spectroscopy and Imaging for Oncological Applications. Photochemistry and Photobiology 1998, 68, 603-632.

6. Foote, C. Definition of type I and type II photosensitized oxidation. Photochemistry and Photobiology 1991, 54, 659-660.

7. Plaetzer, K.; Krammer, B.; Berlanda, J.; Berr, F.; Kiesslich, T. Photophysics and photochemistry of photodynamic therapy: Fundamental aspects. Lasers in Medical Science 2009, 24, 259-268.

8. Moan, J.; Berg, K. The photodegradation of porphyrins in cells can be used to estimate the lifetime of singlet oxygen. Photochemistry and Photobiology 1991, 53, 549-553.

9. Dysart, J.S.; Patterson, M.S. Characterization of Photofrin photobleaching for singlet oxygen dose estimation during photodynamic therapy of MLL cells in vitro. Physics in Medicine and Biology 2005, 50, 2597-2616.

10. Li, K.; Zhang, W.; Fang, H.; Xie, W.; Liu, J.; Zheng, M.; Wang, X.; Wang, W.; Tan, W.; Cheng, $\mathrm{H}$. Superoxide flashes reveal novel properties of mitochondrial reactive oxygen species excitability in cardiomyocytes. Biophysics Journal 2012, 102, 1011-1021.

11. Packer, L.; Cadenas, E., Nitric Oxide: Nitric Oxide, Part E/ed. by Lester Packer. Pt. E. Gulf Professional Publishing: 2005; Vol. 301.

12. Oliveira, C.S.; Turchiello, R.; Kowaltowski, A.J.; Indig, G.L.; Baptista, M.S. Major determinants of photoinduced cell death: Subcellular localization versus photosensitization efficiency. Free Radical Biology and Medicine 2011, 51, 824-833.

13. Castano, A.P.; Demidova, T.N.; Hamblin, M.R. Mechanisms in photodynamic therapy: Part one-Photosensitizers, photochemistry and cellular localization. Photodiagnosis and Photodynamic Therapy 2004, 1, 279-293.

14. Juarranz, Á.; Jaén, P.; Sanz-Rodríguez, F.; Cuevas, J.; González, S. Photodynamic therapy of cancer. Basic principles and applications. Clinical and Translational Oncology 2008, 10, 148154.

15. Igney, F.H.; Krammer, P.H. Death and anti-death: Tumour resistance to apoptosis. Nature Reviews Cancer 2002, 2, 277-288.

16. Mroz, P.; Yaroslavsky, A.; Kharkwal, G.B.; Hamblin, M.R. Cell death pathways in photodynamic therapy of cancer. Cancers 2011, 3, 2516-2539.

17. Hamblin, M.R.; Newman, E.L. New Trends in Photobiology (Invited Review) On the mechanism of the tumour-localising effect in therapy. Journal of Photochemistry and Photobiology B: Biology 1994, 23, 3-8.

18. Maeda, H.; Nakamura, H.; Fang, J. The EPR effect for macromolecular drug delivery to solid tumors: Improvement of tumor uptake, lowering of systemic toxicity, and distinct tumor imaging in vivo. Advanced Drug delivey Reviews 2013, 65, 71-79.

19. Castano, A.P.; Demidova, T.N.; Hamblin, M.R. Mechanisms in photodynamic therapy: Part three - Photosensitizer pharmacokinetics, biodistribution, tumor localization and modes of tumor destruction. Photodiagnosis and Photodynamic Therapy 2005, 2, 91-106.

20. Peng, Q.; Nesland, J.M. Effects of photodynamic therapy on tumor stroma. Ultrastructural Pathology 2004, 28, 333-340. 
21. Dvorak, H.F. How tumors make bad blood vessels and stroma. American Journal of Pathology 2003, 162, 1747-1757.

22. Hofmeister, V.; Schrama, D.; Becker, J.C. Anti-cancer therapies targeting the tumor stroma. Cancer Immunology, Immunotherapy 2008, 57, 1-17.

23. Guo, W.; Giancotti, F.G. Integrin signalling during tumour progression. Nature Reviews Molecular Cell Biology 2004, 5, 816-826.

24. Desgrosellier, J.S.; Cheresh, D.A. Integrins in cancer: Biological implications and therapeutic opportunities. Nature Reviews Cancer 2010, 10, 9-22.

25. Celli, J.P. Stromal interactions as regulators of tumor growth and therapeutic response: A potential target for photodynamic therapy? Israel Journal of Chemistry 2012, 52, 757-766.

26. Chen, B.; Pogue, B.W.; Zhou, X.; O'hara, J.A.; Solban, N.; Demidenko, E.; Hoopes, P.J.; Hasan, T. Effect of Tumor Host Microenvironment on Photodynamic Therapy in a Rat Prostate Tumor Model. Clinical Cancer Research 2005, 720-727.

27. Hanahan, D.; Weinberg, R.A. The Hallmarks of Cancer Review evolve progressively from normalcy via a series of pre. Cell 2000, 100, 57-70.

28. Al-Husein, B.; Abdalla, M.; Trepte, M.; DeRemer, D.L.; Somanath, P.R. Antiangiogenic therapy for cancer: An update. Pharmacotherapy 2012, 32, 1095-1011.

29. Star, W.M.; Marijnissen, H.P.A.; van den Berg-Blok, A.E.; Versteeg, J.A.C.; Franken, K.A.P.; Reinhold, H.S. Destruction of Rat Mammary Tumor and Normal Tissue Microcirculation by Hematoporphyrin Derivative Photoradiation Observed in Vivo in Sandwich Observation Chambers1. Cancer Research 1986, 46, 2532-2540.

30. VH Fingar, P.K.P.H.P.C.M.T.E.A. and T.W. Analysis of acute vascular damage after photodynamictherapy using benzoporphyrin derivative (BPD). British journal of cancer 1999, 79, 1702-1708.

31. Ben-Hur, E.; Heldman, E.; Crane, S.W.; Rosenthal, I. Release of clotting factors from photosensitized endothelial cells: a possible trigger for blood vessel occlusion by photodynamic therapy. FEBS Letter 1988, 236, 105-108.

32. Foster, T.H.; Primavera, M.C.; Marder, V.J.; Hilf, R.; Sporn, L.A. Photosensitized Release of von Willebrand Factor from Cultured Human Endothelial Cells1. Cancer research 1991, 32613266.

33. Nelson', J.S.; Berns , M.W. Tumor destruction in photodynamic therapy. Photochemistry and Photobiology 1987, 46, 829-835.

34. Fingar, V.H.; Wieman, T.J.; Haydon, P.S. The Effects of Thrombocytopenia on Vessel Stasis and Macromolecular Leakage after Photodynamic Therapy Using Photofrin. Photochemistry and Photobiology 1997, 66, 51-54.

35. Kurohane, K.; Tominaga, A.; Sato, K.; North, J.R.; Namba, Y.; Oku, N. Photodynamic therapy targeted to tumor-induced angiogenic vessels. Cancer letters 2001, 167, 49-56.

36. Chen, B.; Pogue, B.W.; Hoopes, P.J.; Hasan, T. Combining vascular and cellular targeting regimens enhances the efficacy of photodynamic therapy. International Journal of Radiation Oncology Biology Physics 2005, 61, 1216-1226.

37. Krzykawska-Serda, M.; Da browski, J.M.; Arnaut, L.G.; Szczygieł, M.; Urbańska, K.; Stochel, G.; Elas, M. The role of strong hypoxia in tumors after treatment in the outcome of bacteriochlorin-based photodynamic therapy. Free Radical Biology and Medicine 2014, 73, 239-251.

38. Castano, A.P.; Mroz, P.; Hamblin, M.R. Photodynamic therapy and anti-tumour immunity. Nature Reviews Cancer 2006, 6, 535-545.

39. Korbelik, M.; Sun, J.; Cecic, I. Photodynamic Therapy-Induced Cell Surface Expression and Release of Heat Shock Proteins: Relevance for Tumor Response. Cancer research 2005, 65, 1018-1026.

40. Compans, R.W.; Georgia, A./; Cooper, M.D.; Ito, A.Y.; Koprowski, S.H.; Pennsylvania', P./; Me1chers, F.; Old Stone, B.M.B.A.; Jolla, L.; California', /; et al. Curren $t$ Topics in Microbiology and Immunology;

41. Amer A. Beg Endogenous ligands of Toll-like receptors: implicationsfor regulating inflammatory and immune response. Trends in immunology 2002, 23, 509-511. 
42. Reginato, E. Immune response after photodynamic therapy increases anti-cancer and antibacterial effects. World Journal of Immunology 2014, $4,1$.

43. Agarwal, M.L.; Larkin, H.E.; Zaidi, S.I.A.; Mukhtar, H.; Oleinick3, N.L. Phospholipase Activation Triggers Apoptosis in Photosensitized Mouse Lymphoma Cells1. Cancer Research 1993, 53, 5897-5902.

44. Garg, A.D.; Nowis, D.; Golab, J.; Agostinis, P. Photodynamic therapy: Illuminating the road from cell death towards anti-tumour immunity. Apoptosis 2010, 15, 19050-1071.

45. Garg, A.D.; Nowis, D.; Golab, J.; Vandenabeele, P.; Krysko, D. v.; Agostinis, P. Immunogenic cell death, DAMPs and anticancer therapeutics: An emerging amalgamation. Biochimica et Biophysica Acta - Reviews on Cancer 2010, 1805, 50-71.

46. Krosll, G.; Korbelik', M.; Dougherty2, G.J. Induction of immune cell infiltration into murine SCCVII tumour by Photofrin-based photodynamic therapy. Journal of Cancer 1995, 549-555.

47. Beltrán Hernández, I.; Yu, Y.; Ossendorp, F.; Korbelik, M.; Oliveira, S. Preclinical and Clinical Evidence of Immune Responses Triggered in Oncologic Photodynamic Therapy: Clinical Recommendations. Journal of Clinical Medicine 2020, 9, 333.

48. Spikes, J.D. New trends in photobiology. Journal of Photochemistry and Photobiology B: Biology 1990, 6, 259-274.

49. Breskey, J.D.; Lacey, S.E.; Vesper, B.J.; Paradise, W.A.; Radosevich, J.A.; Colvard, M.D. Photodynamic Therapy: Occupational Hazards and Preventative Recommendations for Clinical Administration by Healthcare Providers. Photomedicine and Laser Surgery 2013, 31, 398-407.

50. Reynolds, T. Photodynamic Therapy Expands Its Horizons. JNCI Journal of the National Cancer Institute 1997, 89, 112-114.

51. Allison, R.R.; Sibata, C.H. Oncologic photodynamic therapy photosensitizers: A clinical review. Photodiagnosis photodynamic 2010, 7, 61-75.

52. O'Connor, A.E.; Gallagher, W.M.; Byrne, A.T. Porphyrin and nonporphyrin photosensitizers in oncology: Preclinical and clinical advances in photodynamic therapy. Photochemistry and Photobiology 2009, 85, 1053-1074.

53. Ormond, A.B.; Freeman, H.S. Dye sensitizers for photodynamic therapy. Materials 2013, 6 , 817-840.

54. Castano, A.P.; Demidova, T.N.; Hamblin, M.R. Mechanisms in photodynamic therapy: Part one - Photosensitizers, photochemistry and cellular localization. Photodiagnosis and Photodynamic Therapy 2004, 1, 279-293.

55. Rangasamy, S.; Ju, H.; Um, S.; Oh, D.C.; Song, J.M. Mitochondria and DNA Targeting of 5,10,15,20-Tetrakis(7-sulfonatobenzo[b]thiophene) Porphyrin-Induced Photodynamic Therapy via Intrinsic and Extrinsic Apoptotic Cell Death. Journal of Medicinal Chemistry 2015, 58, 6864-6874.

56. Li, Y.; Wang, J.; Zhang, X.; Guo, W.; Li, F.; Yu, M.; Kong, X.; Wu, W.; Hong, Z. Highly water-soluble and tumor-targeted photosensitizers for photodynamic therapy. Organic and Biomolecular Chemistry 2015, 13, 7681-7694.

57. Szaciłowski, K.; Macyk, W.; Drzewiecka-Matuszek, A.; Brindell, M.; Stochel, G. Bioinorganic photochemistry: Frontiers and mechanisms. Chemical Reviews 2005, 105, 2647-2694.

58. Brancaleon, L.; Moseley, H. Laser and Non-laser Light Sources for Photodynamic Therapy. Lasers in Medical Science 2002, 17.

59. Erkiert-Polguj, A.; Halbina, A.; Polak-Pacholczyk, I.; Rotsztejn, H. Light-emitting diodes in photodynamic therapy in non-melanoma skin cancers - own observations and literature review. Journal of Cosmetic and Laser Therapy 2016, 18.

60. Hino, H.; Murayama, Y.; Nakanishi, M.; Inoue, K.; Nakajima, M.; Otsuji, E. 5-Aminolevulinic acid-mediated photodynamic therapy using light-emitting diodes of different wavelengths in a mouse model of peritoneally disseminated gastric cancer. Journal of Surgical Research $\mathbf{2 0 1 3}$, 185.

61. Pariser, D.; Loss, R.; Jarratt, M.; Abramovits, W.; Spencer, J.; Geronemus, R.; Bailin, P.; Bruce, S. Topical methyl-aminolevulinate photodynamic therapy using red light-emitting diode 
light for treatment of multiple actinic keratoses: A randomized, double-blind, placebocontrolled study. Journal of the American Academy of Dermatology 2008, 59.

62. Vaupel, P.; Thews, O.; Hoeckel, M. Introduction Treatment Resistance of Solid Tumors Role of Hypoxia and Anemia. Medical Oncology 2001, 18, 243-259.

63. Fuchs, U.; Thiele, J. The role of oxygen in cutaneous photodynamic therapy. Free Radical Biology and Medicine 1998, 835-847.

64. See, K.L.; Forbes, J.; Betts\$, W.H. Oxygen dependency of phototoxicity with haematoporphyrin derivative. Photochemistry and Photobiology 1984, 39, 631-634.

65. Moan, J.; Berg, K. The photodegradation of porphyrins in cells can be used to estimate the lifetime of singlet oxygen. 1991; Vol. 53, 178-186.

66. Casas, A.; di Venosa, G.; Hasan, T.; Batlle, A. Mechanisms of Resistance to Photodynamic Therapy. Current Medicinal Chemistry 2011, 2486-2515.

67. Fingar, V.H.; Jeffery Wieman, T; Park, Y.J. Henderson, B.W. Implications of a Pre-existing Tumor Hypoxic Fraction on Photodynamic Therapy'. Journal of Surgical Research 1992, 53, 524-528.

68. Al-Waili, N.S.; Butler, G.J.; Beale, J.; Hamilton, R.W.; Lee, B.Y.; Lucas, P. Hyperbaric oxygen and malignancies: a potential role in radiotherapy, chemotherapy, tumor surgery and phototherapy. Medical Science Monitor 2005, 279-289.

69. Chen, Q.; Huang, Z.; Chen, H.; Shapiro, H.; Beckers, J.; Hetzel, F.W. Improvement of Tumor Response by Manipulation of Tumor Oxygenation During Photodynamic Therapy. Photochemistry and Photobiology 2002, 76, 197.

70. Huang, Z.; Chen, Q.; Shakil, A.; Chen, H.; Beckers, J.; Shapiro, H.; Hetzel, F.W. Hyperoxygenation Enhances the Tumor Cell Killing of Photofrin-mediated Photodynamic Therapy. Photochemistry and Photobiology 2003, 78, 496.

71. Delaey, E.; Vandenbogaerde, A.; Merlevede, W.; de Witte, P. Photocytotoxicity of hypericin in normoxic and hypoxic conditions. Journal of Photochemistry and Photobiology B: Biology 2000, 56, 19-24.

72. Price, M.; Heilbrun, L.; Kessel, D. Effects of the oxygenation level on formation of different reactive oxygen species during photodynamic therapy. Photochemistry and Photobiology 2013, 89, 683-686.

73. Benov, L. Photodynamic Therapy: Current Status and Future Directions. Medical Principles and Practice 2015, 24, 14-28.

74. Agostinis, P.; Berg, K.; Cengel, K.A.; Foster, T.H.; Girotti, A.W.; Gollnick, S.O.; Hahn, S.M.; Hamblin, M.R.; Juzeniene, A.; Kessel, D.; et al. Photodynamic therapy of cancer: An update. CA: A Cancer Journal for Clinicians 2011, 61, 250-281.

75. Gill, I.S.; Azzouzi, A.R.; Emberton, M.; Coleman, J.A.; Coeytaux, E.; Scherz, A.; Scardino, P.T. Randomized Trial of Partial Gland Ablation with Vascular Targeted Phototherapy versus Active Surveillance for Low Risk Prostate Cancer: Extended Followup and Analyses of Effectiveness. J. Urol. 2018, 200, 786-793.

76. Weinberg, B.D.; Allison, R.R.; Sibata, C.; Parent, T.; Downie, G. Results of combined photodynamic therapy (PDT) and high dose rate brachytherapy (HDR) in treatment of obstructive endobronchial non-small cell lung cancer (NSCLC). Photodiagnosis and Photodynamic Therapy 2010, 7, 50-58.

77. Ji, W.; Yoo, J.; Bae, E.K.; Lee, J.H.; Choi, C.-M. The effect of Radachlorin ${ }^{\circledR}$ PDT in advanced NSCLC: A pilot study. Photodiagnosis and Photodynamic Therapy 2013, 10, 120-126.

78. Ettinger, D.S.; Akerley, W.; Borghaei, H.; Chang, A.C.; Cheney, R.T.; Chirieac, L.R.; D’Amico, T.A.; Demmy, T.L.; Ganti, A.K.P.; Govindan, R.; et al. Non-Small Cell Lung Cancer. Journal of the National Comprehensive Cancer Network 2012, 10, 1236-1271.

79. Kimura, M.; Miyajima, K.; Kojika, M.; Kono, T.; Kato, H. Photodynamic Therapy (PDT) with Chemotherapy for Advanced Lung Cancer with Airway Stenosis. International Journal of Molecular Sciences 2015, 16, 25466-25475.

80. Chen, K.-C.; Hsieh, Y.-S.; Tseng, Y.-F.; Shieh, M.-J.; Chen, J.-S.; Lai, H.-S.; Lee, J.-M. Pleural Photodynamic Therapy and Surgery in Lung Cancer and Thymoma Patients with Pleural Spread. Plos One 2015, 10, e0133230. 
81. Friedberg, J.S.; Culligan, M.J.; Mick, R.; Stevenson, J.; Hahn, S.M.; Sterman, D.; Punekar, S.; Glatstein, E.; Cengel, K. Radical Pleurectomy and Intraoperative Photodynamic Therapy for Malignant Pleural Mesothelioma. The Annals of Thoracic Surgery 2012, 93, 1658-1667.

82. Cai, X.; Li, W.; Zhang, L.; Wang, X.; Luo, R.; Li, L. Photodynamic therapy for intractable bronchial lung cancer. Photodiagnosis and Photodynamic Therapy 2013, 10, 672-676.

83. Furukawa, K.; Kato, H.; Konaka, C.; Okunaka, T.; Usuda, J.; Ebihara, Y. Locally Recurrent Central-Type Early Stage Lung Cancer \&lt; $1.0 \mathrm{~cm}$ in Diameter After Complete Remission by Photodynamic Therapy. Chest 2005, 128, 3269-3275.

84. Kato, H.; Usuda, J.; Okunaka, T.; Furukawa, K.; Honda, H.; Sakaniwa, N.; Suga, Y.; Hirata, T.; Ohtani, K.; Inoue, T.; et al. Basic and clinical research on photodynamic therapy at Tokyo Medical University Hospital. Lasers in Surgery and Medicine 2006, 38, 371-375.

85. Usuda, J.; Ichinose, S.; Ishizumi, T.; Hayashi, H.; Ohtani, K.; Maehara, S.; Ono, S.; Honda, H.; Kajiwara, N.; Uchida, O.; et al. Outcome of Photodynamic Therapy Using NPe6 for Bronchogenic Carcinomas in Central Airways. Clinical Cancer Research 2010, 16, 2198 2204.

86. Usuda, J.; Ichinose, S.; Ishizumi, T.; Hayashi, H.; Ohtani, K.; Maehara, S.; Ono, S.; Kajiwara, N.; Uchida, O.; Tsutsui, H.; et al. Management of Multiple Primary Lung Cancer in Patients with Centrally Located Early Cancer Lesions. Journal of Thoracic Oncology 2010, 5, 62-68.

87. Bellnier, D.A.; Greco, W.R.; Loewen, G.M.; Nava, H.; Oseroff, A.R.; Pandey, R.K.; Tsuchida, T.; Dougherty, T.J. Population Pharmacokinetics of the Photodynamic Therapy Agent 2-[1Hexyloxyethyl]-2-devinyl Pyropheophorbide-a in Cancer Patients 1. Cancer Research 2003, 63, 1806-1813.

88. Dhillon, S.S.; Demmy, T.L.; Yendamuri, S.; Loewen, G.; Nwogu, C.; Cooper, M.; Henderson, B.W. A Phase I Study of Light Dose for Photodynamic Therapy Using 2-[1-Hexyloxyethyl]-2 Devinyl Pyropheophorbide-a for the Treatment of Non-Small Cell Carcinoma In Situ or NonSmall Cell Microinvasive Bronchogenic Carcinoma: A Dose Ranging Study. Journal of Thoracic Oncology 2016, 11, 234-241.

89. Dougherty, T.J.; Kaufman, J.E.; Goldfarb, A.; Weishaupt, K.R.; Boyle, D.; Mittleman, A. Photoradiation Therapy for the Treatment of Malignant Tumors1. Cancer Research 1978, 2628-2635.

90. Feyh, J. Photodynamic treatment for cancers of the head and neck. Journal of Photochemistry and Photobiology B: Biology 1996, 36, 175-177.

91. Feyh, J.; Goetz, A.; Müller, W.; Königsberger, R.; Kastenbauer, E. Photodynamic therapy in head and neck surgery. Journal of Photochemistry and Photobiology B: Biology 1990, 7, 353358.

92. Ikeda, H.; Tobita, T.; Ohba, S.; Uehara, M.; Asahina, I. Treatment outcome of Photofrin-based photodynamic therapy for $\mathrm{T} 1$ and $\mathrm{T} 2$ oral squamous cell carcinoma and dysplasia. Photodiagnosis and Photodynamic Therapy 2013, 10, 229-235.

93. Dtlkes, M.G.; DeJode, M.L.; Gardiner, Q.; Kenyon, G.S.; McKelvie, P. Treatment of head and neck cancer with photodynamic therapy: results after one year. The Journal of Laryngology \& Otology 1995, 109, 1072-1076.

94. Copper, M.P.; Tan, I.B.; Oppelaar, H.; Ruevekamp, M.C.; Stewart, F.A. Metatetra(hydroxyphenyl)chlorin Photodynamic Therapy in Early-Stage Squamous Cell Carcinoma of the Head and Neck. Archives of Otolaryngology-Head \& Neck Surgery 2003, 129, 709.

95. Rigual, N.; Shafirstein, G.; Cooper, M.T.; Baumann, H.; Bellnier, D.A.; Sunar, U.; Tracy, E.C.; Rohrbach, D.J.; Wilding, G.; Tan, W.; et al. Photodynamic Therapy with 3-(1'-Hexyloxyethyl) Pyropheophorbide a for Cancer of the Oral Cavity. Clinical Cancer Research 2013, 19, 66056613.

96. Shafirstein, G.; Rigual, N.R.; Arshad, H.; Cooper, M.T.; Bellnier, D.A.; Wilding, G.; Tan, W.; Merzianu, M.; Henderson, B.W. Photodynamic therapy with 3-(1'-hexyloxyethyl) pyropheophorbide-a for early-stage cancer of the larynx: Phase Ib study. Head \& Neck 2016, 38, 377-383.

97. Hopper, C. Photodynamic therapy: a clinical reality in the treatment of cancer. The Lancet Oncology 2000, 1, 212-219. 
98. Lou, P.-J.; Jäger, H.R.; Jones, L.; Theodossy, T.; Bown, S.G.; Hopper, C. Interstitial photodynamic therapy as salvage treatment for recurrent head and neck cancer. British Journal of Cancer 2004, 91, 441-446.

99. Kübler, A.C.; de Carpentier, J.; Hopper, C.; Leonard, A.G.; Putnam, G. Treatment of squamous cell carcinoma of the lip using Foscan-mediated Photodynamic Therapy. International Journal of Oral and Maxillofacial Surgery 2001, 30, 504-509.

100. Stoker, S.D.; Indrasari, S.R.; Herdini, C.; Hariwiyanto, B.; Karakullukcu, B.; Dhamiyati, W.; Widayati, K.; Romdhoni, A.C.; Fles, R.; Haryana, S.M.; et al. Photodynamic therapy as salvage therapy for patients with nasopharyngeal carcinoma experiencing local failures following definitive radiotherapy. Photodiagnosis and Photodynamic Therapy 2015, 12, 519-525.

101. Jerjes, W.; Upile, T.; Hamdoon, Z.; Nhembe, F.; Bhandari, R.; Mackay, S.; Shah, P.; Mosse, C.A.; AS Brookes, J.; Morley, S.; et al. Ultrasound-guided photodynamic therapy for deep seated pathologies: prospective study. Lasers in Surgery and Medicine 2009, 41, 612-621.

102. Tan, I.B.; Dolivet, G.; Ceruse, P.; Poorten, V. vander; Roest, G.; Rauschning, W. Temoporfinmediated photodynamic therapy in patients with advanced, incurable head and neck cancer: A multicenter study. Head \& Neck 2010, 32, 1597-1604.

103. vander Poorten, V.; Meulemans, J.; Nuyts, S.; Clement, P.; Hermans, R.; Hauben, E.; Delaere, P. Postoperative photodynamic therapy as a new adjuvant treatment after robot-assisted salvage surgery of recurrent squamous cell carcinoma of the base of tongue. World Journal of Surgical Oncology 2015, 13, 214.

104. Caesar, L.; van Doeveren, T.E.M.; Tan, I.B.; Dilci, A.; van Veen, R.L.P.; Karakullukcu, B. The use of photodynamic therapy as adjuvant therapy to surgery in recurrent malignant tumors of the paranasal sinuses. Photodiagnosis and Photodynamic Therapy 2015, 12, 414-421.

105. Bown, S.G. Photodynamic therapy for cancer of the pancreas. Gut 2002, 50, 549-557.

106. Stummer, W.; Pichlmeier, U.; Meinel, T.; Wiestler, O.D.; Zanella, F.; Reulen, H.-J. Fluorescence-guided surgery with 5-aminolevulinic acid for resection of malignant glioma: a randomised controlled multicentre phase III trial. The Lancet Oncology 2006, 7, 392-401.

107. Stepp, H.; Beck, T.; Pongratz, T.; Meinel, T.; Kreth, F.-W.; Tonn, J.Ch.; Stummer, W. ALA and Malignant Glioma: Fluorescence-Guided Resection and Photodynamic Treatment. Journal of Environmental Pathology, Toxicology and Oncology 2007, 26, 157-164.

108. Eljamel, M.S.; Goodman, C.; Moseley, H. ALA and Photofrin ${ }^{\circledR}$ Fluorescence-guided resection and repetitive PDT in glioblastoma multiforme: a single centre Phase III randomised controlled trial. Lasers in Medical Science 2008, 23, 361-367.

109. Muller, P.J.; Wilson, B.C. Photodynamic therapy of brain tumors - A work in progress. Lasers in Surgery and Medicine 2006, 38, 384-389.

110. Akimoto, J.; Haraoka, J.; Aizawa, K. Preliminary clinical report on safety and efficacy of photodynamic therapy using talaporfin sodium for malignant gliomas. Photodiagnosis and Photodynamic Therapy 2012, 9, 91-99.

111. Muragaki, Y.; Akimoto, J.; Maruyama, T.; Iseki, H.; Ikuta, S.; Nitta, M.; Maebayashi, K.; Saito, T.; Okada, Y.; Kaneko, S.; et al. Phase II clinical study on intraoperative photodynamic therapy with talaporfin sodium and semiconductor laser in patients with malignant brain tumors. Journal of Neurosurgery 2013, 119, 845-852.

112. Stupp, R.; Mason, W.P.; van den Bent, M.J.; Weller, M.; Fisher, B.; Taphoorn, M.J.B.; Belanger, K.; Brandes, A.A.; Marosi, C.; Bogdahn, U.; et al. Radiotherapy plus Concomitant and Adjuvant Temozolomide for Glioblastoma. New England Journal of Medicine 2005, 352, 987-996.

113. Fink, C.; Enk, A.; Gholam, P. Photodynamic therapy - Aspects of pain management. JDDG: Journal der Deutschen Dermatologischen Gesellschaft 2015, 13, 15-22.

114. Yano, T.; Muto, M.; Minashi, K.; Iwasaki, J.; Kojima, T.; Fuse, N.; Doi, T.; Kaneko, K.; Ohtsu, A. Photodynamic therapy as salvage treatment for local failure after chemoradiotherapy in patients with esophageal squamous cell carcinoma: A phase II study. International Journal of Cancer 2012, 131, 1228-1234.

115. Yano, T.; Muto, M.; Yoshimura, K.; Niimi, M.; Ezoe, Y.; Yoda, Y.; Yamamoto, Y.; Nishisaki, H.; Higashino, K.; Iishi, H. Phase I study of photodynamic therapy using talaporfin sodium and 
diode laser for local failure after chemoradiotherapy for esophageal cancer. Radiation Oncology 2012, 7, 113.

116. de Visscher, S.A.H.J.; Melchers, L.J.; Dijkstra, P.U.; Karakullukcu, B.; Tan, I.B.; Hopper, C.; Roodenburg, J.L.N.; Witjes, M.J.H. mTHPC-mediated Photodynamic Therapy of Early Stage Oral Squamous Cell Carcinoma: A Comparison to Surgical Treatment. Annals of Surgical Oncology 2013, 20, 3076-3082.

117. Abrahamse, H.; Hamblin, M.R. New photosensitizers for photodynamic therapy. Biochemical Journal 2016, 473, 347-364.

118. Shafirstein, G.; Bellnier, D.; Oakley, E.; Hamilton, S.; Potasek, M.; Beeson, K.; Parilov, E. Interstitial Photodynamic Therapy-A Focused Review. Cancers 2017, 9, 12.

119. Dolmans, D.E.J.G.J.; Kadambi, A.; Hill, J.S.; Flores, K.R.; Gerber, J.N.; Walker, J.P.; Rinkes, I.H.M.B.; Jain, R.K.; Fukumura, D. Targeting Tumor Vasculature and Cancer Cells in Orthotopic Breast Tumor by Fractionated Photosensitizer Dosing Photodynamic Therapy; 2002; Vol. 62.

120. Bugaj, A.M. Targeted photodynamic therapy - a promising strategy of tumor treatment. Photochemical \& Photobiological Sciences 2011, 10, 1097.

121. Mew, D.; Wat, C.K.; Towers, G.H.; Levy, J.G. Photoimmunotherapy: treatment of animal tumors with tumor-specific monoclonal antibody-hematoporphyrin conjugates. The Journal of Immunology 1983, 130, 1473.

122. Vandongen, G.; Visser, G.; Vrouenraets, M. Photosensitizer-antibody conjugates for detection and therapy of cancer. Advanced Drug Delivery Reviews 2004, 56, 31-52.

123. Kameyama, N.; Matsuda, S.; Itano, O.; Ito, A.; Konno, T.; Arai, T.; Ishihara, K.; Ueda, M.; Kitagawa, Y. Photodynamic Therapy Using an Anti-EGF Receptor Antibody Complexed with Verteporfin Nanoparticles: A Proof of Concept Study. Cancer Biotherapy and Radiopharmaceuticals 2011, 26, 697-704.

124. Mitsunaga, M.; Ogawa, M.; Kosaka, N.; Rosenblum, L.T.; Choyke, P.L.; Kobayashi, H. Cancer cell-selective in vivo near infrared photoimmunotherapy targeting specific membrane molecules. Nature Medicine 2011, 17, 1685-1691.

125. Nakajima, T.; Sano, K.; Choyke, P.L.; Kobayashi, H. Improving the Efficacy of Photoimmunotherapy (PIT) using a Cocktail of Antibody Conjugates in a Multiple Antigen Tumor Model. Theranostics 2013, 3, 357-365.

126. van Driel, P.B.A.A.; Boonstra, M.C.; Slooter, M.D.; Heukers, R.; Stammes, M.A.; Snoeks, T.J.A.; de Bruijn, H.S.; van Diest, P.J.; Vahrmeijer, A.L.; van Bergen en Henegouwen, P.M.P.; et al. EGFR targeted nanobody-photosensitizer conjugates for photodynamic therapy in a preclinical model of head and neck cancer. Journal Controlled Release 2016, 229, 93-105.

127. Deken, M.M.; Kijanka, M.M.; Beltrán Hernández, I.; Slooter, M.D.; de Bruijn, H.S.; van Diest, P.J.; van Bergen en Henegouwen, P.M.P.; Lowik, C.W.G.M.; Robinson, D.J.; Vahrmeijer, A.L.; et al. Nanobody-targeted photodynamic therapy induces significant tumor regression of trastuzumab-resistant HER2-positive breast cancer, after a single treatment session. $J$. Controlled Release 2020, 323, 269-281.

128. Shi, Q.; Tao, Z.; Yang, H.; Fan, Q.; Wei, D.; Wan, L.; Lu, X. PDGFR $\beta$-specific affibodydirected delivery of a photosensitizer, IR700, is efficient for vascular-targeted photodynamic therapy of colorectal cancer. Drug Delivery 2017, 24, 1818-1830.

129. Thurber, G.M.; Schmidt, M.M.; Wittrup, K.D. Antibody tumor penetration: Transport opposed by systemic and antigen-mediated clearance. Advanced Drug Delivery Reviews 2008, 60, $1421-1434$.

130. St Denis, T.G.; Hamblin, M.R. Synthesis, bioanalysis and biodistribution of photosensitizer conjugates for photodynamic therapy. Bioanalysis 2013, 5, 1099-1114.

131. Duska, L.; Hamblin, M.; Bamberg, M.; Hasan, T. Biodistribution of charged F(ab')2 photoimmunoconjugates in a xenograft model of ovarian cancer. British Journal of Cancer 1997, 75, 837-844.

132. Kuimova, M.K.; Bhatti, M.; Deonarain, M.; Yahioglu, G.; Levitt, J.A.; Stamati, I.; Suhling, K.; Phillips, D. Fluorescence characterisation of multiply-loaded anti-HER2 single chain Fv- 
photosesitizer conjugates suitable for photodynamic therapy. Photochemical \& Photobiological Sciences 2007, 6,933.

133. Staneloudi, C.; Smith, K.A.; Hudson, R.; Malatesti, N.; Savoie, H.; Boyle, R.W.; Greenman, J. Development and characterization of novel photosensitizer: $\mathrm{scFv}$ conjugates for use in photodynamic therapy of cancer. Immunology 2007, 120, 512-517.

134. Bhatti, M.; Yahioglu, G.; Milgrom, L.R.; Garcia-Maya, M.; Chester, K.A.; Deonarain, M.P. Targeted photodynamic therapy with multiply-loaded recombinant antibody fragments. International Journal of Cancer 2007, 122, 1155-1163.

135. Milgrom, L.R. Towards recombinant antibody-fragment targeted Photodynamic Therapy. Science Progress 2008, 91, 241-263.

136. Oliveira, S.; van Dongen, G.A.M.S.; Walsum, M.S.; Roovers, R.C.; Stam, J.C.; Mali, W.; van Diest, P.J.; van Bergen en Henegouwen, P.M.P. Rapid Visualization of Human Tumor Xenografts through Optical Imaging with a Near-Infrared Fluorescent Anti-Epidermal Growth Factor Receptor Nanobody. Molecular Imaging 2012, 11, 33-46.

137. Heukers, R.; van Bergen en Henegouwen, P.M.P.; Oliveira, S. Nanobody-photosensitizer conjugates for targeted photodynamic therapy. Nanomedicine Nanotechnology 2014, 10,14411451 . 


\section{Chapter 2}

Nanobody-targeted photodynamic therapy selectively kills viral GPCR-expressing glioblastoma cells

Timo W.M. De Groof ${ }^{\dagger}$, Vida Mashayekhi ${ }^{\dagger}$, Tian Shu Fan, Nick D. Bergkamp, Javier Sastre Toraño, Jeffrey R. van Senten, Raimond Heukers, Martine J. Smit ${ }^{\ddagger}$, and Sabrina Oliveira

$\dagger, \ddagger$ equal contribution

Molecular pharmaceutics, 2019; 16(7): 3145-3156

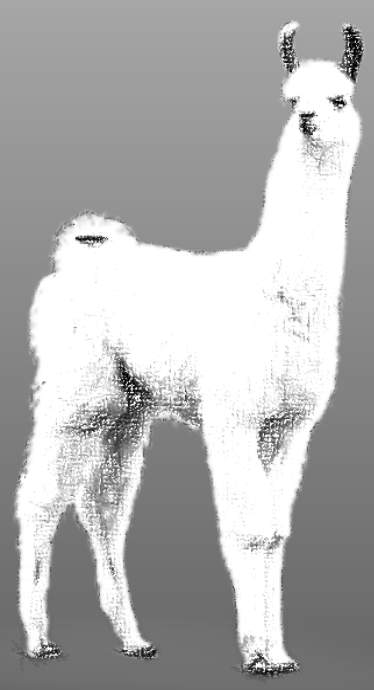




\section{ABSTRACT}

Photodynamic therapy (PDT) eradicates tumors by the local activation of a photosensitizer with near-infrared light. One of the aspects hampering the clinical use of PDT is the poor selectivity of the photosensitizer. To improve this, we have recently introduced a new approach for targeted PDT by conjugating photosensitizers to nanobodies. Diverse G protein-coupled receptors (GPCRs) show aberrant overexpression in tumors and are therefore interesting targets in cancer therapy. Here we show that GPCR-targeting nanobodies can be used in targeted PDT. We have developed a nanobody binding the extracellular side of the viral GPCR US28, which is detected in tumors like glioblastoma. The nanobody was site-directionally conjugated to the water-soluble photosensitizer IRDye700DX. This nanobody-photosensitizer conjugate selectively killed US28expressing glioblastoma cells both in 2D and 3D cultures upon illumination with nearinfrared light. This is the first example employing a GPCR as target for nanobody-directed PDT. With the emerging role of GPCRs in cancer, this data provides a new angle for exploiting this large family of receptors for targeted therapies. 


\section{Introduction}

Photodynamic therapy (PDT) is a minimally invasive modality where cancer cells are eradicated through local activation of a photosensitizer, by means of near infrared light. Activation of the photosensitizer leads to the production of singlet oxygen species, which have detrimental effects on proteins, lipids and nucleic acids, resulting in cell toxicity, vascular responses, and additional inflammatory responses [1]. However, one of the main aspects hampering the use of PDT in the clinic, is the hydrophobicity of the photosensitizer and its poor selectivity. This leads to off-target effects, the need to wait 2-4 days between administration of the photosensitizer and light application, and photosensitivity several weeks post PDT [1,2]. To improve this, more hydrophilic photosensitizers have been generated and/or other approaches like nanoparticles have been used for photosensitizer delivery [3-5]. In addition, photosensitizers have successfully been conjugated to antibodies directed against tumor antigens [6,7]. Currently, a phase I clinical study, involving the water-soluble photosensitizer IRDye700DX conjugated to an epidermal growth factor receptor (EGFR) targeting antibody is ongoing, for head and neck cancer [8]. The conjugation of a photosensitizer to monoclonal antibodies has increased the selectivity, showing promising results, but the large size of these antibody-photosensitizer conjugates impedes efficient tumor penetration and has slow clearance [9-11]. As alternative, we have introduced nanobody-targeted PDT, for more effective tumor penetration and faster clearance of the conjugates $[12,13]$.

Nanobodies are antibody-fragments derived from heavy-chain antibodies from Camelidae family members, which can be generated by immunization of llamas/alpacas with an antigen of interest [14]. Nanobodies display low immunogenicity, are highly soluble and physically stable, and have a ten-fold lower molecular weight (12-15 kDa), compared to conventional antibodies. This enables enhanced tumor penetration and the ability to bind cryptic antigenic sites inaccessible for conventional antibodies [15-17]. In previous studies, nanobodies targeting the epidermal growth factor receptor (EGFR), were successfully conjugated with the water-soluble photosensitizer IRDye700DX and used for targeted PDT in vitro and in vivo resulting in selective toxicity to EGFR-overexpressing tumor cells and extensive tumor damage $[12,13]$.

$\mathrm{G}$ protein-coupled receptors (GPCRs) are a family of receptors that play a prominent role in multiple physiological processes and are involved in multiple diseases, 
including cancer [18-20]. In several types of cancers, GPCR overexpression and/or dysregulated signaling contributes to angiogenesis, metastasis and/or tumor growth [2123]. These findings have led to an increasing interest in targeting GPCRs in cancer. To date, several GPCR-targeting nanobodies have already shown therapeutic potential in cancer, by inhibiting GPCR signaling [24-29]. Alternatively, such nanobodies could serve as ideal moieties for guiding functional groups, including photosensitizers, towards cancer cells.

Herpesviruses also contain genes encoding for GPCRs with high homology to human chemokine receptors. The human cytomegalovirus (HCMV) is a human herpesvirus with an estimated seroprevalence of approximately 50 to $90 \%$ of the worldwide population [30,31]. HCMV and US28, one of the four HCMV-encoded viral GPCRs, have been detected in multiple tumors, including gliomas, colorectal cancer and prostate cancer [3238]. In particular, US28 activates oncogenic signaling pathways and displays an oncomodulatory role in the progression of tumors like glioblastoma [27,32,33,39-41]. We recently developed an US28-targeting nanobody, which partially inhibits this US28enhanced tumor growth in vitro and in vivo by inhibiting constitutive US28 signaling [27]. Since US28 is a foreign viral target expressed in tumors, but not in the surrounding healthy tissue, US28 would be an ideal target for selective therapies, including nanobody-targeted PDT.

The aim of this research was to eradicate US28-expressing glioblastoma cells using nanobody-targeted PDT. For this, we have selected a new nanobody that binds a discontinuous epitope of US28 with high affinity. We have conjugated the water-soluble photosensitizer IRDye700DX to an unpaired cysteine in a C-terminal tag of the nanobody without compromising the binding affinity. Notably, we were able to selectively kill US28expressing glioblastoma cells in 2D cultures, as well as 3D spheroids. These findings show the potential of GPCR-targeting nanobodies in nanobody-directed PDT.

\section{Experimental section DNA constructs}

The pVUN014 phagemid vector was a gift from Prof. Dr. H.J. de Haard (argenx $\mathrm{BV}$, Zwijnaarde, Belgium). The pET28a vector for periplasmic production of nanobodies in E. coli was described previously [42]. The pcDEF3 vector was a gift from Dr. J.A. 
Langer [43]. Genes encoding the different US28 mutants (US28- $\Delta 2-22$ ) were either described previously or were ordered from Eurofins (Ebersberg, Germany) [44].

\section{Cell culture}

HEK293T cells and U251 cells were purchased from ATCC (Wesel, Germany). Doxycycline-inducible US28 expression in U251 cells (U251-iUS28) and in HEK293T cells (HEK293T-iUS28) were described previously [27]. To induce US28 expression, cells were induced with doxycycline $(1 \mathrm{ug} / \mathrm{ml}$, D9891, Sigma-Aldrich, Saint Louis, Missouri, USA) for $48 \mathrm{~h}$. Cells were grown at $5 \% \mathrm{CO}_{2}$ and $37{ }^{\circ} \mathrm{C}$ in Dulbecco's Modified Eagle's Medium (Thermo Fisher Scientific, Waltham, Massachusetts, USA) supplemented with 1\% Penicillin/Streptomycin (Thermo Fisher Scientific) and 10\% Fetal Bovine Serum (FBS, Thermo Fisher Scientific). FBS was heat inactivated (30 min, $60^{\circ}$ C) for the culturing of U251 cells.

\section{Transfection of adherent cells}

Two million HEK293T cells were plated in a $10 \mathrm{~cm}^{2}$ dish (Greiner Bio-one, Kremsmunster, Austria). The next day, cells were transfected with $100 \mathrm{ng}$ of the different pcDEF3-US28 constructs and adjusted with empty pcDEF3 DNA to a total of $5 \mu \mathrm{g}$ DNA and $30 \mu \mathrm{g} 25 \mathrm{kDa}$ linear polyethyleneimine (Sigma-Aldrich) in $150 \mathrm{mM} \mathrm{NaCl}$ solution, resulting in a DNA:PEI ratio of 1:6. The DNA-PEI mixture was vortexed for 10 seconds and incubated for $15 \mathrm{~min}$ at room temperature (RT). Subsequently the mixture was added dropwise to the adherent HEK293T cells.

\section{Membrane extract preparation}

To obtain membrane extracts, HEK293T-iUS28 or U251-iUS28 cells were induced with doxycycline as described above. Cells were washed with cold PBS and resuspended afterwards in cold PBS. Cells were centrifuged at $1500 \mathrm{~g}$ at $4{ }^{\circ} \mathrm{C}$. Pellet was resuspended in cold PBS and again centrifuged at $1500 \mathrm{~g}$ at $4{ }^{\circ} \mathrm{C}$. The pellet was resuspended in membrane buffer $(15 \mathrm{mM}$ Tris-Cl, $0.3 \mathrm{mM}$ EDTA, $2 \mathrm{mM}$ $\mathrm{MgCl}_{2}, \mathrm{pH} 7.5$ ) and disrupted by the Dounce Homogenizer Potter-Elvehjem at $1200 \mathrm{~g}$.

\section{Llama immunization and phage display library construction}

Two llamas were immunized using the pcDEF3 vector encoding for VHL/E US28. DNA was injected a total of 8 times. Of these, 4 subcutaneous injections occurred in 
one stretch with 2-week intervals, which was followed by a lag-period of 5 weeks. These injections were followed up by two sets of boost injections, each consisting of 2 injections with a 2-week interval. One week after the final injections, blood was drawn, and peripheral blood mononuclear cells were collected from both llamas and total RNA was isolated. cDNA was obtained by reverse transcription-PCR using the SuperScriptTM ${ }^{\mathrm{IV}}$ First-Strand Synthesis System (Invitrogen, Carlsbad, California, USA). Genes, encoding for the variable domains of the heavy-chain only antibodies, of both llamas were amplified using PCR and cloned into the pVUN014 phagemid vector and transformed into electrocompetent E. coli TG1 (Lucigen), to make 2 libraries. Library sizes were estimated by means of a serial dilution of transformants. Different clones were picked, and colony PCR was performed using DreamTaq polymerase (Thermo Fisher Scientific) to determine the amount of clones containing a nanobody insert. The same PCR product was also cut with MvaI FastDigest (Thermo Fisher Scientific) to determine the diversity of clones in the library.

\section{Phage production}

At the start of each selection round, 10 times the size of both nanobody libraries were pooled together (for round 1) or the rescues of the previous selection round (for round 2 and 3) were diluted in 2xTY broth containing $100 \mu \mathrm{g} / \mathrm{ml}$ ampicillin (Melford Biolabs ltd., Ipswich, UK) and 2\% (w/v) glucose and grown until $\mathrm{OD}_{600}$ of 0.5. Cultures were infected with VCSM13 helper phage (Stratagene, San Diego, California, USA) at phagebacteria ratio of 10:1-20:1. Cultures were grown $30 \mathrm{~min}$ without shaking followed by 30 min with shaking at $37{ }^{\circ} \mathrm{C}$. Bacteria were centrifuged at $4500 \mathrm{~g}$ and the pellet was resuspended in 2xTY broth containing $50 \mu \mathrm{g} / \mathrm{ml}$ kanamycin (Melford Biolabs ltd.) and $100 \mu \mathrm{g} / \mathrm{ml}$ ampicillin. The culture was grown overnight at $28{ }^{\circ} \mathrm{C}$ to allow phage production. Next day, the culture was centrifuged at $4500 \mathrm{~g}$ and supernatant was added to ice-cold 20\% PEG6000/2.5 M NaCl (ratio 4:1) and incubated for $30 \mathrm{~min}$ on ice. The supernatant was centrifuged at $4000 \mathrm{~g}$ and the phage pellet was resuspended in PBS. The phage solution was centrifuged, and the supernatant was again added to ice-cold 20\% PEG6000/2.5 $\mathrm{M} \mathrm{NaCl}$ and incubated for $10 \mathrm{~min}$ on ice. The supernatant was centrifuged again, and the phage pellet was resuspended in PBS. 


\section{Phage display selections}

To obtain US28 specific binding nanobodies, 3 rounds of phage selections were performed using membrane extracts of the inducible US28 (VHL/E strain) HEK293T or U251 cell lines. Fifty $\mu \mathrm{g}$ of the membrane extracts were coated in a 96 well MicroWell $^{\mathrm{TM}}$ MaxiSorp ${ }^{\mathrm{TM}}$ flat bottom plate (Sigma-Aldrich) overnight at $4{ }^{\circ} \mathrm{C}$. Wells were washed 3 times with PBS and blocked with 2\% (w/v) skimmed milk (Sigma-Aldrich) in PBS for $1 \mathrm{~h}$ at RT. Phages were diluted 1:10 (round 1) or 1:100 (round 2 and 3) in $0.2 \%(\mathrm{w} / \mathrm{v})$ skimmed milk in PBS and added to the wells for $2 \mathrm{~h}$ at RT while shaking. If a counter-selection was performed during round 3, phages were first incubated with $250 \mu \mathrm{g}$ of U251 membrane extracts for $1 \mathrm{~h}$ head-over-head at RT. The mix was centrifuged at $4000 \mathrm{~g}$ and the supernatant was added to the wells containing the membrane extracts of the induced US28 U251 cell line. After phage incubation, wells were washed 20 times with PBS with an incubation step of 10 min on a shaker each 5th washing step. Phages were eluted with $10 \mathrm{mg} / \mathrm{ml}$ Trypsin (Sigma-Aldrich) for $30 \mathrm{~min}$ at RT and the eluate was mixed with $4 \mathrm{mg} / \mathrm{ml}$ 4-benzenesulfonyl fluoride hydrochloride (Sigma-Aldrich). Eluted phages were rescued by infecting TG1 cells $\left(\mathrm{OD}_{600}\right.$ of 0.5$)$ and grown overnight at $37^{\circ} \mathrm{C}$. Rescued phages were used for subsequent rounds of phage display. After 2 and 3 rounds of selections, bacteria were plated, and single colonies were grown in a 96 wells plate containing 2xTY broth and $100 \mu \mathrm{g} / \mathrm{ml}$ ampicillin.

\section{Phage Enzyme-Linked Immunosorbent Assay}

Single colonies, picked after the second or third round of selections, were grown in $2 \times T Y$ broth and $100 \mu \mathrm{g} / \mathrm{ml}$ ampicillin at $37^{\circ} \mathrm{C}$. When cultures were grown until $\mathrm{OD}_{600}$ of 0.5 , the bacteria were infected with VCSM13 helper phage (final concentration $\left.3.75 \times 10^{13} \mathrm{pfu} / \mathrm{ml}\right)$. Cultures were grown $30 \mathrm{~min}$ without shaking followed by $30 \mathrm{~min}$ with shaking at $37{ }^{\circ} \mathrm{C}$. $2 \times \mathrm{TY}$ broth and $100 \mu \mathrm{g} / \mathrm{ml}$ ampicillin and kanamycin were added to obtain a final concentration of $50 \mu \mathrm{g} / \mathrm{ml}$ kanamycin. Cultures were grown overnight at $28^{\circ} \mathrm{C}$. Twenty-five $\mu \mathrm{g}$ of the membrane extracts with or without US28 were coated in a 96 well MicroWell ${ }^{\mathrm{TM}}$ MaxiSorp ${ }^{\mathrm{TM}}$ flat bottom plate overnight at $4{ }^{\circ} \mathrm{C}$. Next day, wells were washed 3 times with PBS and blocked with 3\% (w/v) skimmed milk in PBS for $1 \mathrm{~h}$ at RT. Phage cultures were centrifuged at $4000 \mathrm{~g}$ and supernatant was added to $3 \%(\mathrm{w} / \mathrm{v})$ skimmed milk in a 1:1 ratio and incubated for $1 \mathrm{~h}$ at RT on a shaker. Blocked phage solution was added to the MaxiSorp ${ }^{\mathrm{TM}}$ flat bottom plate containing membrane extracts with 
and without US28. Phages were incubated for $2 \mathrm{~h}$ at RT on a shaker. Wells were washed 5 times with PBS. Mouse-anti-M13 ${ }^{\text {HRP }}$ (GE-Healthcare, Chicago, Illinois, USA) was diluted 1:5000 in 3\% (w/v) skimmed milk in PBS and incubated for $1 \mathrm{~h}$ at RT while shaking. The plates were washed again 5 times with PBS. O-phenylenediamine (OPD) solution (2 mM OPD; Sigma-Aldrich, $35 \mathrm{mM}$ citric acid, $66 \mathrm{mM} \mathrm{Na} \mathrm{HPO}_{4}, 0.015 \% \mathrm{H}_{2} \mathrm{O}_{2}$, $\mathrm{pH}$ 5.6) was added to the wells and the reaction was stopped with $1 \mathrm{M} \mathrm{H}_{2} \mathrm{SO}_{4}$. Optical density was measured at $490 \mathrm{~nm}$ with a PowerWave plate reader (BioTek, Winooski, Vermont, USA) and the ratio of binding to the membrane extracts with and without US28 was determined.

\section{Nanobody production}

Nanobody gene fragments were recloned in frame with a myc-His6 tag in the pET28a production vector and E. coli BL21 were transformed by means of heat shock. Nanobodies were produced as described previously [25]. Purity of the nanobodies was verified by sodiumdodecyl sulfate-polyacrylamide gel electrophoresis (SDS-PAGE) under reducing conditions (Bio-Rad, Hercules, California, USA).

\section{ELISA binding assay}

HEK293T or U251 membrane extracts (20-50 $\mu \mathrm{g})$ with or without US28 were coated in a 96 well MicroWell ${ }^{\mathrm{TM}}$ MaxiSorp $^{\mathrm{TM}}$ flat bottom plate overnight at $4{ }^{\circ} \mathrm{C}$. Next day, wells were washed 3 times with PBS and blocked with 2\%(w/v) skimmed milk in PBS for $1 \mathrm{~h}$ at RT. Nanobodies were diluted in $2 \%(\mathrm{w} / \mathrm{v})$ skimmed milk and incubated for $1 \mathrm{~h}$ at RT on a shaker. During the competition binding ELISA, $20 \mathrm{nM}$ VUN100 was coincubated with previously described $100 \mathrm{nM}$ untagged trivalent US28 nanobody or untagged trivalent irrelevant nanobody [27]. Nanobodies were detected with mouse-antiMyc antibody (1:1000, Clone 9B11, Cell Signaling Technology, Leiden, The Netherlands) and horseradish peroxidase (HRP)-conjugated goat-anti-mouse antibody (1:1000, BioRad). US28 expression was determined by means of rabbit-anti US28 antibody (Covance, Denver, USA, 1:2000, described previously) [41] and goat-anti-rabbit HRP-conjugated antibody (1:1000, Bio-Rad). All antibodies were diluted in $2 \%(\mathrm{w} / \mathrm{v})$ skimmed milk and incubated for $1 \mathrm{~h}$ on a shaker at RT. Between each incubation step, wells were washed 3 times with PBS. After the last incubation steps, wells were washed 3 times with PBS and OPD was added to the wells and the reaction was stopped with $1 \mathrm{M} \mathrm{H}_{2} \mathrm{SO}_{4}$. Optical density 
was measured at $490 \mathrm{~nm}$ with a PowerWave plate reader (BioTek). Data was analyzed using GraphPad Prism version 7.0 (GraphPad Software, Inc., La Jolla, CA, USA).

\section{Competition binding}

Membrane extracts of HEK293T and HEK293T overexpressing US28 were used during competition binding studies. The experiments were performed as described previously [27]. Data was analyzed using GraphPad Prism version 7.0.

\section{Phospholipase $\mathbf{C}$ activation assay}

The activation of phospholipase $\mathrm{C}$ was assessed as described previously and data was analyzed using GraphPad Prism version 7.0 [41].

\section{Immunofluorescence microscopy}

Transiently transfected HEK293T or (US28-overexpressing) U251 cells were seeded in poly-L-lysine (Sigma-Aldrich) coated 96 well plates and were grown at $37{ }^{\circ} \mathrm{C}$ and $5 \% \mathrm{CO}_{2}$. Cells were prepared for immunofluorescence microscopy as described previously [25]. Briefly, cells were fixed with 4\% paraformaldehyde (Sigma-Aldrich) for $10 \mathrm{~min}$ at RT and subsequently permeabilized with $0.5 \%$ NP-40 (Sigma-Aldrich) for $30 \mathrm{~min}$ at RT. Nanobodies were incubated for $1 \mathrm{~h}$ at RT and detected using Mouse-antiMyc antibody (1:1000, 9B11 clone, Cell Signaling). US28 was visualized with the rabbit anti-US28 antibody (1:1000, Covance) [41]. Subsequently, cells were washed and incubated with Goat-anti-Rabbit Alexa Fluor 546 (1:1000 in 1\% (v/v) FBS /PBS, Thermo Fisher Scientific) and Goat-anti-Mouse Alexa Fluor 488 (1:1000 in 1\% (v/v) FBS/PBS, Thermo Fisher Scientific). When binding of VUN100 to CX3CR1 was assessed, receptor expression was detected using rat-anti-HA antibody (1:1000 in 1\% (v/v) FBS/PBS, Clone 3F10, Roche) or rabbit-anti-HA antibody (1:1000 in 1\% (v/v) FBS/PBS, H6908, SigmaAldrich) and Goat-anti-Rat Alexa Fluor 546 (1:1000 in 1\% (v/v) FBS /PBS, Thermo Fisher Scientific) or Goat-anti-Rabbit Alexa Fluor 546 (1:1000 in 1\% (v/v) FBS/PBS, Thermo Fisher Scientific). Cells were visualized with an Olympus FSX-100 microscope.

\section{ELISA for US28 expression}

Transiently transfected HEK293T were seeded in poly-L-lysine (Sigma-Aldrich) coated 96 well plates and were grown at $37{ }^{\circ} \mathrm{C}$ and $5 \% \mathrm{CO}_{2}$. Cells were fixed with 4\% paraformaldehyde (Sigma-Aldrich) for $10 \mathrm{~min}$ at RT. To assess total receptor expression, cells were subsequently permeabilized with $0.5 \%$ NP-40 (Sigma-Aldrich) for 
$30 \mathrm{~min}$ at RT. Cells were blocked for $30 \mathrm{~min}$ at RT in $1 \%$ (v/v) FBS/PBS. US28 constructs were detected with a rat-anti-HA antibody (1:1000 in $1 \%(\mathrm{v} / \mathrm{v}) \mathrm{FBS} / \mathrm{PBS}$, Clone 3F10, Roche). Subsequently, wells were washed and incubated with HRP-conjugated goat-antirat antibody (1:1000 in 1\% (v/v) FBS/PBS, Pierce). All antibodies were incubated for $1 \mathrm{~h}$ on a shaker at RT. Between each incubation step, wells were washed 3 times with PBS. After the last incubation steps, wells were washed 3 times with PBS and OPD was added to the wells and the reaction was stopped with $1 \mathrm{M} \mathrm{H}_{2} \mathrm{SO}_{4}$. Optical density was measured at $490 \mathrm{~nm}$ with a PowerWave plate reader (BioTek). Data was analyzed using GraphPad Prism version 7.0 (GraphPad Software, Inc., La Jolla, CA, USA).

\section{Immunohistochemistry}

The experiments were performed as described previously [27]. US28 expression was detected using polyclonal rabbit-anti-US28 antibody (1:700, Covance) while nanobody binding was detected using mouse-anti-Myc antibody (1:500, 9B11 clone, Cell Signaling). MACH2 Universal HRP-Polymer detection was used as secondary antibody (Biocare Medical, Pacheco, California, USA).

\section{Nanobody-photosensitizer conjugates}

The nanobody gene was re-cloned into a pET28a vector to add a C-terminal cysteine (VUN100-Cys) for subsequent modification. Production was performed as described previously and the nanobody was purified using chromatography (ÄKTAxpress) and $1 \mathrm{ml}$ Histrap FF crude column (GE Healthcare) and $5 \mathrm{ml}$ HiTrap Desalting column (GE Healthcare). The VUN100-Cys was incubated with $20 \mathrm{mM}$ tris(2-carboxyethyl)phosphine (TCEP) at RT for $15 \mathrm{~min}$. The buffer was replaced with $50 \mathrm{mM}$ sodium phosphate containing $500 \mathrm{mM} \mathrm{NaCl}$ and $1 \mathrm{mM}$ EDTA using Zeba spin desalting column (Thermo Fisher Scientific). The VUN100-Cys concentration was determined with the NanoDrop spectrophotometer (NanoDrop Technologies, Wilmington, Delaware, USA) at $280 \mathrm{~nm}$. Immediately after buffer exchange, the VUN100-Cys $(1 \mathrm{mg} / \mathrm{ml})$ was mixed with 3 molar equivalents of the photosensitizer IRDye700DX-maleimide and incubated overnight at $4{ }^{\circ} \mathrm{C}$ on a rotator. The next day, the free photosensitizer was removed by passing the solution through 3 consecutive Zeba spin desalting columns which were pre-equilibrated with $2 \mathrm{M} \mathrm{NaCl}$ in PBS. The degree of conjugation and concentration of the protein was determined as described previously [12]. The purity and the integrity of the nanobody- 
photosensitizer conjugate was determined on SDS-PAGE gel. The gel was imaged on an Odyssey Infrared scanner at $700 \mathrm{~nm}$ (LI-COR Biosciences, Lincoln, Nebraska, USA).

\section{LC-MS of nanobody-photosensitizer conjugates}

Intact nanobody-photosensitizer conjugates were analyzed with ultra-high performance liquid chromatography mass-spectrometry (UHPLC-MS). The system consisted of a 1290 Infinity UHPLC-UV system (Agilent Technologies, Waldbronn, Germany) connected to an Agilent Technologies 6560 ion mobility quadrupole time-offlight mass spectrometer with a jet stream electrospray ionization interface, operated in positive ion mode. Separation was achieved using an Acquity UPLC protein BEH C4 column (50 x 2.1 mm, $300 \AA$, $1.7 \mu \mathrm{m}$ particles, Waters, Milford, Massachusetts, USA), which was maintained at $70{ }^{\circ} \mathrm{C}$ during analysis. A $1 \mu \mathrm{l}$ sample volume was injected into the system and analytes were separated using linear gradient elution with $0.1 \%$ formic acid (solvent A) and $0.1 \%$ formic acid:acetonitrile 5:95 (v/v) (solvent B), increasing from $20-40 \% \mathrm{~B}$ in $10 \mathrm{~min}$ at a flow rate of $0.30 \mathrm{ml} / \mathrm{min}$. Detection was performed with UV at $280 \mathrm{~nm}$ and MS using a capillary voltage of $5.5 \mathrm{kV}$, a nozzle voltage of $2 \mathrm{kV}$, a nitrogen nebulizing pressure of $45 \mathrm{psi}$, a nitrogen sheath gas flow of $11 \mathrm{l} / \mathrm{min}$ at $400{ }^{\circ} \mathrm{C}$ and a drying gas flow of $8 \mathrm{l} / \mathrm{min}$ at $350{ }^{\circ} \mathrm{C}$. Data were acquired between $\mathrm{m} / \mathrm{z} \mathrm{300-3200} \mathrm{and}$ processed using Agilent Technologies MassHunter software (version B.08.00).

\section{Determination of the expression of US28 receptor}

To determine the number of US28 expressing U251 cells upon induction with doxycycline for $48 \mathrm{~h}$, US28 positive cells were seeded in a 96-well plate. Cells were fixed with 4\% PFA for $10 \mathrm{~min}$ at RT and then incubated with $100 \mathrm{mM}$ glycine for $10 \mathrm{~min}$ at RT, subsequently permeabilized with $0.5 \%$ Triton X-100 for $10 \mathrm{~min}$ at RT. The cells were blocked with $2 \%$ BSA for $30 \mathrm{~min}$ at RT. Cells were incubated with polyclonal rabbit antiUS28 primary antibody (1:1000 diluted in blocking buffer) for $1 \mathrm{~h}$ at RT. After multiple washing steps, goat anti rabbit Alexa 488 secondary antibody (1:1000 diluted in blocking buffer) and TO-PRO ${ }^{\circledR}-3(1 \mu \mathrm{M}$, Thermo Fisher Scientific) were added to the cells and incubated for $1 \mathrm{~h}$ at RT. The cells were imaged with an EVOS microscope and analyzed with ImageJ. The US28 expressing cells were detected by antibody staining, and the percentage was calculated related to the total cell number (detected with TO-PRO ${ }^{\circledR}-3$ ). 


\section{Cell binding assay with nanobody-photosensitizer}

U251-iUS28 were induced for $48 \mathrm{~h}$ with doxycycline resulting in U251 cells overexpressing US28 (US28 positive) and the control (US28 negative) cells (if not induced) as described earlier [45]. US28 positive and US28 negative cells were seeded at 8000 cells per well in a 96-well plate (Nunc, Roskilde, Denmark). The next day, cells were washed once with binding medium (DMEM without phenol red, $25 \mathrm{mM}$ HEPES and $1 \%$ BSA, pH 7.4). Subsequently, different concentrations of nanobody-photosensitizer were added to the plate and incubated for $2 \mathrm{~h}$ at $4{ }^{\circ} \mathrm{C}$. Unbound nanobody-photosensitizer conjugate was removed by washing 3 times with binding buffer. The amount of bound nanobody-photosensitizer was detected with Odyssey infrared scanner (Li-COR) at $700 \mathrm{~nm}$. Data was analyzed using GraphPad Prism version 7.0.

\section{In vitro PDT}

The US28 positive and negative U251 cells were washed with washing medium (DMEM medium without phenol red, 10\% FBS, 1\% Penicillin/Streptomycin). The cells were incubated with different concentrations of nanobody-photosensitizer for $1 \mathrm{~h}$ at $37^{\circ} \mathrm{C}$. Cells were washed 2 times with washing medium and bound and/or internalized nanobodyphotosensitizer was detected using the Odyssey infrared scanner at $700 \mathrm{~nm}$. Next, cells were illuminated $33 \mathrm{~min}$ with $5 \mathrm{~mW} / \mathrm{cm}^{2}$ fluence rate for a total light dose of $10 \mathrm{~J} / \mathrm{cm}^{2}$ using a $690 \mathrm{~nm}$ diode laser through a $600 \mu \mathrm{m}$ optic fiber (Modulight, Tampere, Finland). After overnight incubation of the cells at $37^{\circ} \mathrm{C}$, the viability of the cells was assessed by AlamarBlue $^{\circledR}$ reagent, as recommended by manufacturer (Bio-Rad). Cell viability was measured with a Fluostar Optima fluorescent plate reader (BMG Labtech GmbH, Ortenberg, Germany). Cells that were neither illuminated nor treated were used to determine $100 \%$ cell viability. The percentage of cell viability was calculated relative to the untreated cells and data was analyzed using GraphPad Prism version 7.0.

\section{Co-culture assay}

The US28 positive and negative U251 cells were co-seeded in a 96-well plate in various ratios. After $1 \mathrm{~h}$ of incubation with $50 \mathrm{nM}$ of nanobody-photosensitizer, the cells were illuminated with total light dose of $10 \mathrm{~J} / \mathrm{cm}^{2}$. After overnight incubation of the cells at $37{ }^{\circ} \mathrm{C}$, cells were incubated with propidium iodide $(1 \mu \mathrm{g} / \mathrm{ml}$, Invitrogen) and calcein AM $\left(0.5 \mu \mathrm{g} / \mathrm{ml}\right.$, Invitrogen) for $10 \mathrm{~min}$ at $37{ }^{\circ} \mathrm{C}$. The cells were imaged with an EVOS 
microscope and counted with ImageJ. The theoretical percentage of positive cells versus percentage of dead cells was plotted.

\section{In vitro PDT in 3D spheroids}

The US28 positive and negative U251 cells were seeded in ultra-low attachment U bottom 96 well plate (Corning). Two days after seeding, $50 \mu 1$ of the medium was removed from each well and spheroids were incubated with different concentrations of nanobodyphotosensitizer in washing medium for $1 \mathrm{~h}$ at $37^{\circ} \mathrm{C}$. After 3 times washing of the spheroids with the same medium, the plate was illuminated 33 min with $5 \mathrm{~mW} / \mathrm{cm}^{2}$ fluence rate for a total light dose of $10 \mathrm{~J} / \mathrm{cm}^{2}$ using a $690 \mathrm{~nm}$ diode laser through a $600 \mu \mathrm{m}$ optic fiber (Modulight). After overnight incubation at $37^{\circ} \mathrm{C}$, the viability was assessed by CellTiterGlo $^{\circledR}$ 3D reagent as recommended by manufacturer (Promega, Madison, Wisconsin, USA). The percentage of cell viability was calculated relative to the untreated cells. Data was analyzed using GraphPad Prism version 7.0.

\section{Statistical analysis}

Statistical significance was determined by unpaired Student's t test. Significant values were set as indicated in figure legends.

\section{Results}

\section{Selection and characterization of a new US28 nanobody}

In this study, we set out to develop a novel US28-targeting nanobodyphotosensitizer conjugate to eradicate US28-expressing tumor cells via targeted PDT. This approach requires a nanobody with high affinity and specificity for US28. Because of the relatively poor binding affinity of the monovalent US28 nanobody published earlier [27], we developed new nanobodies with higher affinity for US28. Phage libraries with nanobody genes were constructed after immunization of llamas with US28 DNA. Upon panning selections, 330 clones were screened for selective binding to US28 by means of a phage ELISA. Of these 330 screened clones, 85 were positive for specific binding to US28 and could be divided in 7 different groups based on their CDR3 regions. Interestingly, one of the US28-binding nanobody clones contained a similar CDR3 as the previously published US28 nanobody [27]. This new nanobody was named VUN100 and further characterized. Importantly, VUN100 bound US28 of membranes obtained from US28- 
overexpressing cells with a binding affinity of $2 \pm 1 \mathrm{nM}$, which is approximately 170-fold higher than the previously reported US28 nanobody (US28 NB) $(340 \pm 80 \mathrm{nM})$ (Figure 1A). To ensure selectivity, binding of VUN100 to US28 was compared to the binding of an irrelevant NB (binding to the azodye reactive red 6, RR6) (Figure S1A) and binding of VUN100 to the chemokine receptor CX3CR1, with which US28 shares the highest homology, were assessed (Figure S1B). No binding of the irrelevant nanobody to US28 was seen, and VUN100 did not show any binding to CX3CR1. VUN100 displaced ${ }^{125}$ I-CCL5 with a Ki value of $6 \pm 1 \mathrm{nM}$, compared to $142 \pm 49 \mathrm{nM}$ for the previous US28 nanobody (Figure 1B and C and Table 1). Similarly, VUN100 displaced ${ }^{125}$ I-CX3CL1 with a potency of $6 \pm 1 \mathrm{nM}$, compared to $100 \pm 58 \mathrm{nM}$ for the previous US28 nanobody. This improvement in potency of approximately a 20 -fold was in line with the increased binding affinity of VUN100 for US28. Despite this increase in affinity, VUN100 did not affect the US28 constitutive activity (Figure 1D). As the previous monovalent US28 NB, VUN100 did not show any inverse agonistic properties while the previously reported bivalent US28 NB was able to partially inhibit US28 signaling [27]. In conclusion, new immunizations and selections yielded a new US28 targeting nanobody with a superior binding affinity and potency in chemokine displacement. 
A

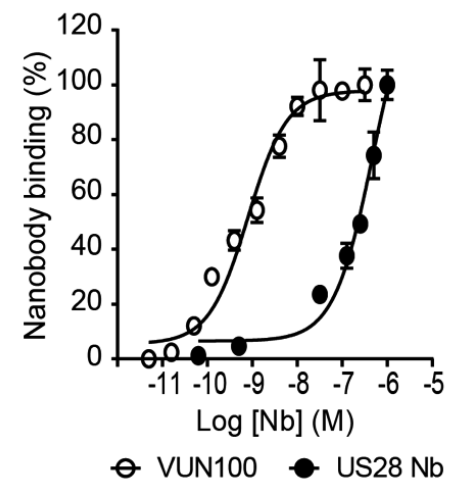

C

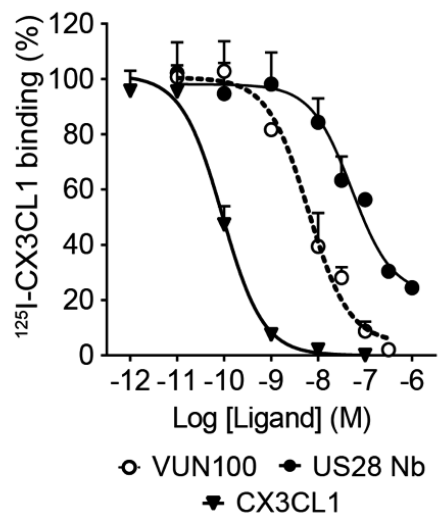

B

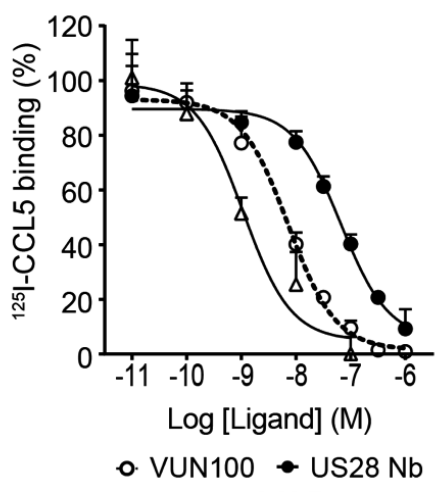

Figure 1. VUN100 binds the HCMV-encoded US28 with high affinity. A) Binding of the nanobodies VUN100 and US28 NB to US28-expressing membranes, as determined by ELISA. B-C) Displacement of ${ }^{125} \mathrm{I}-\mathrm{CCL} 5$ (B) and ${ }^{125} \mathrm{I}$-CX3CL1 (C) from US28-expressing membranes by unlabeled ligand or the nanobodies VUN100 and US28 NB. D) Effect of nanobodies on the US28mediated phospholipase C activation. No NB: No nanobody; Irr. NB.: Irrelevant nanobody; Biv. US28 NB: bivalent US28 nanobody.

Table 1. Pharmacological characteristics of nanobodies targeting US28.

\begin{tabular}{l|l|c|l} 
Nanobody & $\mathrm{k}_{\mathrm{D}}(\mathrm{nM}, \pm \mathrm{SD})$ & $\mathrm{K}_{\mathrm{i}} \operatorname{CCL} 5(\mathrm{nM}, \pm \mathrm{SD})$ & $\mathrm{K}_{\mathrm{i}} \operatorname{CX3CL1}(\mathrm{nM}, \pm \mathrm{SD})$ \\
\hline US28 Nb & $340 \pm 80$ & $142 \pm 49$ & $100 \pm 58$ \\
VUN100 & $2 \pm 1$ & $6 \pm 1$ & $6 \pm 1$
\end{tabular}




\section{VUN100 binds to the N-terminus and ECL3 of US28}

In order to determine which domains of US28 are essential for binding of VUN100, binding was assessed on US28 mutants in immunofluorescence microscopy. While clear binding of VUN100 to US28 wild type (WT) was observed, binding of VUN100 was lost when the first 22 amino acids of the N-terminus of US28 were removed (Figure 2A). This was also observed for the previously published US28 nanobody [27]. This observation, together with the similarity in CDR3 regions, suggest that both nanobodies bind to a similar epitope of US28. The US28 NB was able to displace binding of VUN100 to US28, further corroborating that they bind a similar region of US28 (Figure S2A). To study the binding epitope of VUN100 in more detail, binding of VUN100 was assessed on US28 mutants with point mutations in the N-terminus. Mutations of the amino acids to alanines at position 11 to 15 did not have any effect on the binding of VUN100 (Figure S2B). Interestingly, mutation of the tyrosine at position 16 to a phenylalanine (US28 Y16F) resulted in impaired binding of VUN100. This suggests that this tyrosine is important for binding of VUN100 to US28. Since nanobodies are known to bind discontinuous epitopes, US28 extracellular loop (ECL)-mutants were constructed. Due to the homology of CCR5 with US28 and CCL5 binding to both receptors, chimeric receptors were constructed in which the ECLs of US28 were replaced with the corresponding ECLs of CCR5. To ensure proper folding and expression of the chimeric receptors, the (surface) expression was confirmed by ELISA (Figure 2B). Next, binding of VUN100 to these chimeras was assessed (Figure 2C). The substitution of the ECL1 (US28 ECL1-CCR5) and ECL2 (US28 ECL2-CCR5) did not influence the binding of VUN100. However, the substitution of the ECL3 (US28 ECL3-CCR5) resulted in the loss of binding of VUN100. These results were further confirmed by determining the binding affinity on membrane extracts expressing the different US28 mutants (Figure 2D). These data indicate that VUN100 binds a discontinues epitope on the extracellular side of US28 that involves tyrosine 16 in the N-terminus and ECL3. 
A

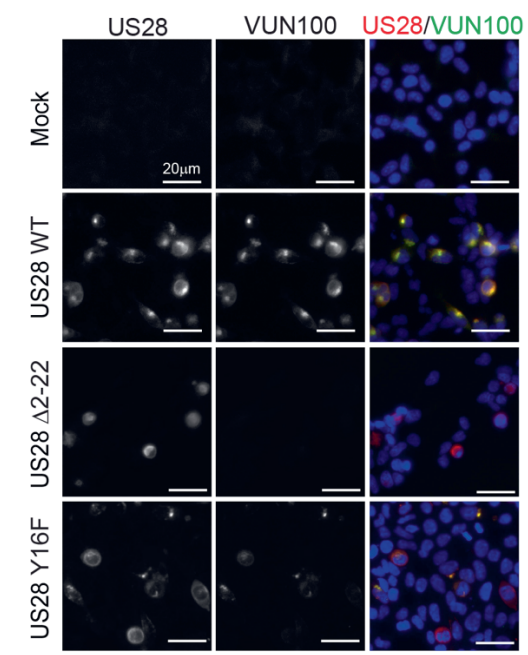

C

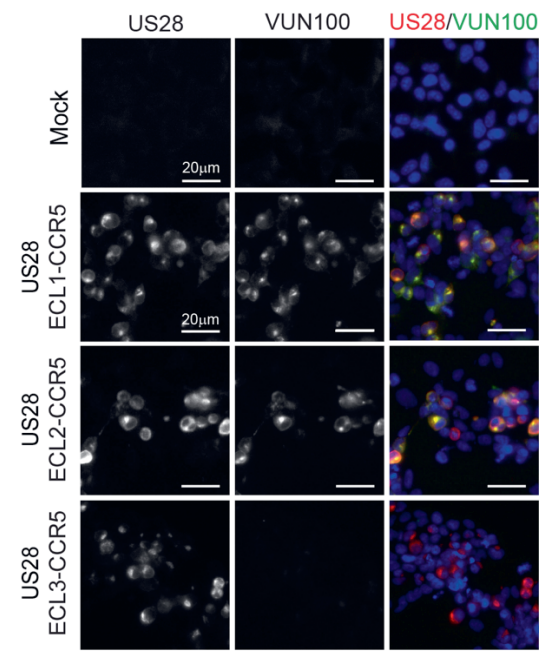

B
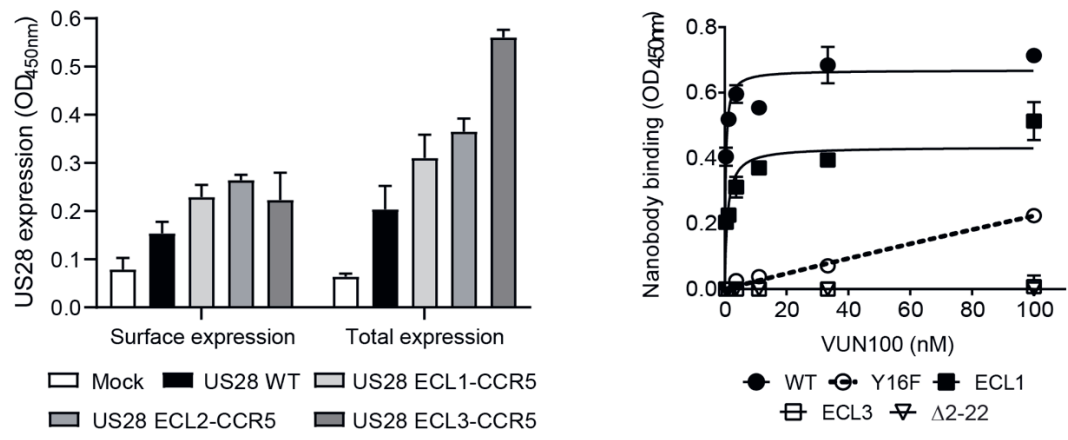

Figure 2. VUN100 binds to the N-terminus and ECL3 loop of US28. A) Immunofluorescence microscopy of the binding of VUN100 to Mock, US28 wild-type (US28 WT), N-terminus truncated US28 (US28 $\Delta 2-22$ ) and US28 with mutation of the tyrosine at position 16 to a phenylalanine (US28 Y16F). US28 was detected using an anti-US28 antibody (US28). VUN100 binding was detected using the Myc-tag and an anti-Myc antibody (VUN100). B) Detection of surface and total expression of HA-tagged US28 wildtype (US28 WT) and HA-tagged US28 chimeras with the CL1-3 loop being substituted by the corresponding loops of CCR5. Receptor expression was detected by the N-terminal HA-tag. C) Immunofluorescence microscopy of the binding of VUN100 to Mock transfected or US28 chimeras with the ECL1-3 loop being substituted by the corresponding loops of CCR5. US28 was detected using an anti-US28 antibody (US28). VUN100 binding was detected using the Myc-tag and an anti-Myc antibody (VUN100). D) Binding ELISA of different concentrations of VUN100 to membranes of HEK293T cells transfected with wild-type US28 (WT), US28 Y16F (Y16F), US28 ECL1-CCR5 chimera (ECL1), US28 ECL3-CCR5 chimera (ECL3) and US28 $\Delta 2-22(\Delta 2-22)$.

In US28-expressing glioblastoma cells, VUN100 was also able to bind US28 (Figure 3A). US28 has been detected in multiple cancers, of which US28 expression in glioblastoma is the most widely studied. Therefore, detection of US28 in glioblastoma 
sections of HCMV-infected glioblastoma patients by these nanobodies was assessed. A comparable US28 expression pattern in glioblastoma was detected by both the polyclonal anti-US28 antibody directed against the C-terminus of US28 and the two US28 targeting nanobodies (Figure 3B).

Taken together, the newly selected anti-US28 nanobody VUN100 shows high affinity for the extracellular domains of US28 and binds to US28 in HCMV-positive glioblastoma tissues. This makes VUN100 therefore a suitable targeting moiety for US28targeted therapies.

A

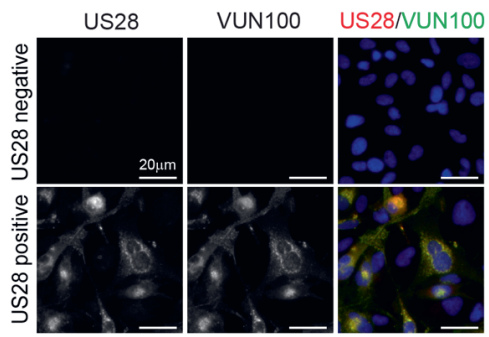

B

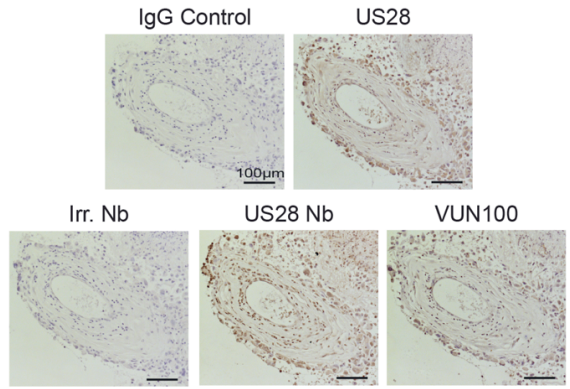

Figure 3. VUN100 binds US28 in glioblastoma cells and glioblastoma patient material. A) Immunofluorescence microscopy of the binding of VUN100 to glioblastoma cells (US28 negative) and glioblastoma cells expressing US28 (US28 positive). US28 was detected using an anti-US28 antibody (US28). VUN100 binding was detected using the Myc-tag and an anti-Myc antibody (VUN100). B) Detection of US28 in parallel sections of glioblastoma patient material. Nuclei were stained using Hoechst staining (blue).US28 was detected using an anti-US28 antibody (US28). Nanobodies were detected via their Myc-tag (brown). An IgG isotype control and irrelevant nanobody (Irr. NB) were used as controls.

\section{Site-directed conjugation of IRDye700DX to VUN100}

To facilitate the specific killing of US28-expressing tumor cells by PDT, the water-soluble photosensitizer IRDye700DX was conjugated to VUN100. Previously, this conjugation was done for other nanobodies through random labeling of lysine residues using NHS-coupling $[12,13]$. However, conjugation to lysines in VUN100 led to a loss of binding capacity to US28 (Figure S3A). This is likely due to presence of lysines in and near the CDR regions of VUN100. To resolve this, a VUN100 variant with an additional cysteine in a C-terminal tag (VUN100-Cys) was produced. The addition of this cysteine did not have any effect on the affinity of the nanobody to US28 (Figure S3B) but enabled sitedirectional conjugation of the photosensitizer to VUN100 by maleimide-coupling. Site- 
directed conjugation and purification resulted in a VUN100-PS conjugate of $\sim 15 \mathrm{kDa}$ with a degree of conjugation (DOC) of 0.7 molecules of photosensitizer per nanobody and less than $2 \%$ of free photosensitizer (Figure 4A). Besides the VUN100-PS conjugate, small amounts of other fluorescent products were detected on the SDS-PAGE, which are likely impurities conjugated to the photosensitizer. VUN100-PS was also analyzed by UHPLCMS, providing separation of conjugated and unconjugated nanobody and identification by their deconvoluted mass. The mass difference between unconjugated $(15.2 \mathrm{kDa})$ and conjugated nanobody $(17.1 \mathrm{kDa})$ corresponded well to the mass of the photosensitizer $(1.9 \mathrm{kDa})$, confirming the conjugation of one nanobody with a single photosensitizer molecule (Figure S4). The percentage area of conjugated nanobody in the chromatograms, with respect to total nanobody area, was $71.3 \%$ with UV and $71.8 \%$ with MS detection which corresponded well with the obtained DOC of 0.7. Directional conjugation of the photosensitizer to the nanobody did not affect its binding capacity, as binding of VUN100PS to US28 positive cells and not to negative cells was observed by immunofluorescence microscopy (Figure 4B). In addition, VUN100-PS bound US28 positive cells with a binding affinity of $3.1 \pm 0.1 \mathrm{nM}$, while no specific binding was seen on US28 negative cells (Figure 4C). These results indicate that the site-directed conjugation of the photosensitizer to VUN100 was successful and did not change the binding properties of VUN100 to US28.

A

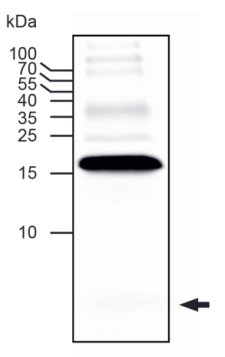

B

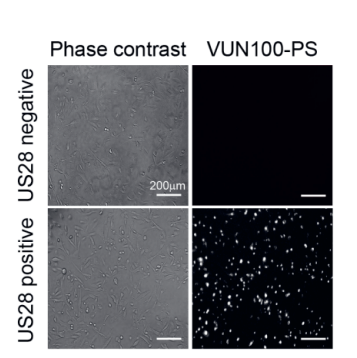

C

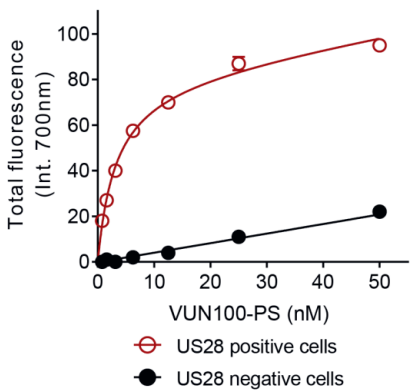

Figure 4. Binding of VUN100-PS conjugates to US28. A) SDS-PAGE of the VUN100-IRDye 700DX conjugate (VUN100-PS). A small quantity of free photosensitizer is observed at the gel front (arrow). B) Binding of VUN100-PS to US28 negative (US28 negative) and US28 positive U251 glioblastoma cells (US28 positive). U251-iUS28 were induced for $48 \mathrm{~h}$ with doxycycline resulting in US28 positive glioblastoma cells US28 negative glioblastoma cells (if not induced). VUN100-PS was visualized with a widefield fluorescent microscope. C) Binding of different concentrations of VUN100-PS to US28 negative and positive cells on ice. Fluorescence of VUN100-PS bound to cells was detected using an Odyssey infrared scanner at $700 \mathrm{~nm}$. 


\section{VUN100-targeted PDT selectively kills US28 positive cells}

Next, the ability of the VUN100-PS conjugate to kill US28 positive cells was assessed. First, the percentage of US28 positive cells upon induction with doxycycline was quantified. Immunofluorescence staining showed that $89 \pm 3 \%$ of the cell population was US28 positive after 48 hours of induction (Figure 5A). Next, the effect of VUN100-PS on US28 positive and negative cells was assessed. During a pulse of $1 \mathrm{~h}$ at $37{ }^{\circ} \mathrm{C}$ VUN100-PS associated specifically with the US28 positive cells (Figure 5B). One day after the activation of the photosensitizer by a light dose of $10 \mathrm{~J} / \mathrm{cm}^{2}$, cell viability was determined. US28-targeted PDT resulted in up to $90 \%$ reduction in cell viability of US28 positive cells with an $\mathrm{EC}_{50}$ value of $1.1 \pm 0.2 \mathrm{nM}$ (Figure 5C). These percentages of cytotoxicity correlated well with the percentage of US28-expressing cells upon induction by doxycycline as shown in Figure 5A. The selective cell killing by VUN100-targeted PDT was confirmed by staining with propidium iodide (dead cells) and calcein (living cells). Staining of propidium iodide in cells correlated well with the VUN100-PS binding, indicating that only those cells expressing US28 and able to bind VUN100-PS died (Figure 5D). The few viable cells that remained did not show association of VUN100-PS or propidium iodide staining. 
A

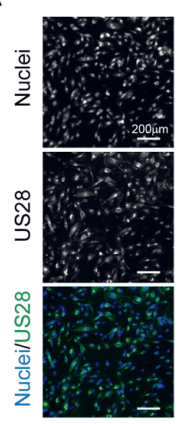

B

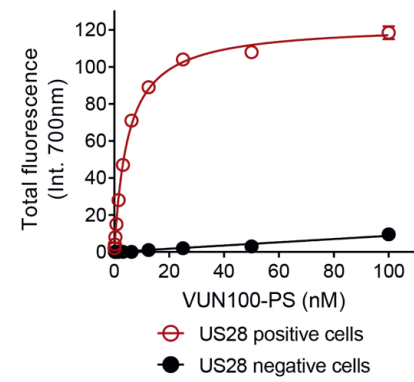

C

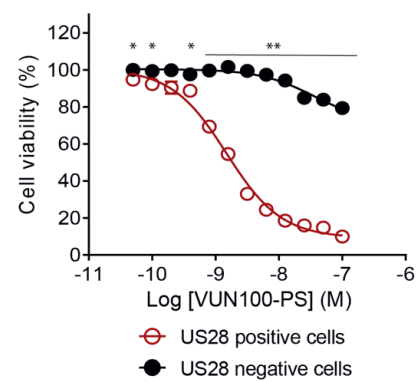

\section{$\mathrm{D}$}
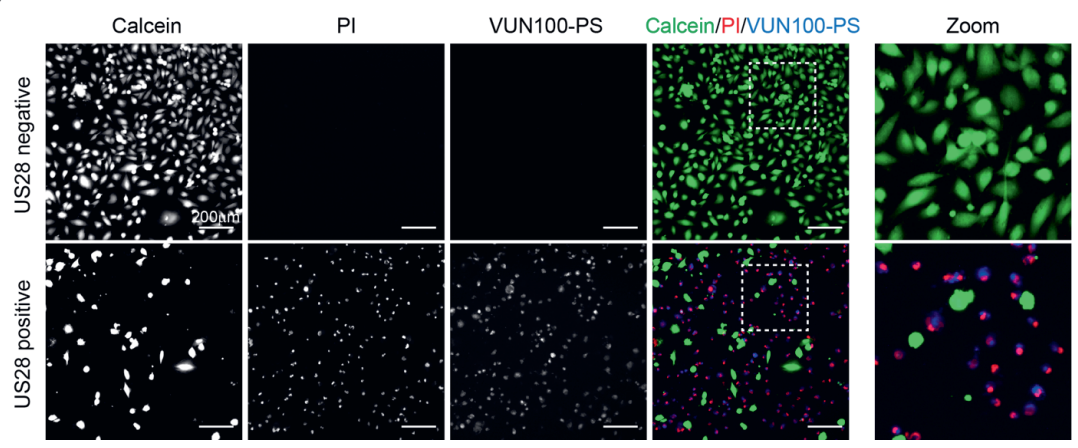

Figure 5. VUN100-PS selectively kills US28-expressing cells upon illumination. A) Staining of US28 after $48 \mathrm{~h}$ of doxycycline-induction of US28-expressing glioblastoma cells. US28 was visualized using anti-US28 antibody and the percentage of US28-positive cells was determined using ImageJ. B) Detection of different concentrations of bound and internalized VUN100 to US28 positive and negative cells. Binding was determined using Odyssey infrared scanner at $700 \mathrm{~nm}$. C) Determination of cell viability after incubation with different concentrations of VUN100-PS and illumination $10 \mathrm{~J} / \mathrm{cm}^{2}$ light dose. Cell viability was determined using AlamarBlue ${ }^{\circledR}$ reagent $(* \mathrm{p}<0.05 ; * * \mathrm{p}<0.01, \mathrm{t}$ test). D) Staining of dead cells with propidium iodide (PI) and living cells (calcein) $24 \mathrm{~h}$ after nanobody-targeted PDT using $50 \mathrm{nM}$ VUN100-PS, performed as described above.

To further determine the selectivity and local effect of nanobody-targeted PDT, co-culture experiments with different ratios of US28 positive and US28 negative cells were performed. Even in the case of decreasing number of US28 positive cells, a clear decrease in number of killed cells is observed, suggesting VUN100-PS targeted PDT killed the US28 positive cells and did not affect the US28 negative cells (Figure S5). This confirms that, in close proximity of US28 negative and positive cells, the short activity range of the activated photosensitizer allows the selective killing of targeted cells, while leaving the negative cells unaffected. 


\section{VUN100-targeted PDT efficiently induces cell toxicity in US28 expressing 3D spheroids}

To test the efficacy of VUN100-targeted PDT in a more relevant setting, its effect was tested on 3D spheroid cultures of US28 expressing and US28 negative glioblastoma cells. After 2 days of seeding, both types of spheroids were viable (Figure 6A). After $1 \mathrm{~h}$ of incubation with VUN100-PS, association of VUN100-PS to the US28 positive spheroids was observed, while no fluorescence of VUN100-PS was observed for the US28 negative spheroids (Figure 6B). Next, spheroids were illuminated with near-infrared light and cell viability was assessed. In line with the results from the 2D culture experiments, VUN100PS selectively induced cell death in up to $90 \%$ of the cells in the US28 positive spheroids with an $\mathrm{EC}_{50}$ value of $4.1 \pm 1.6 \mathrm{nM}$, while no cell death was observed in the US28 negative spheroids (Figure 6C).

A

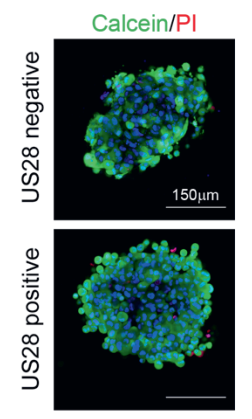

B
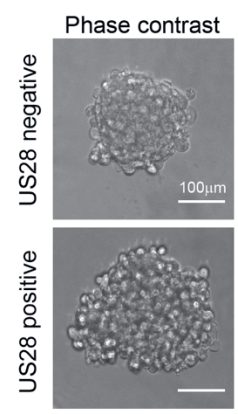

VUN100-PS

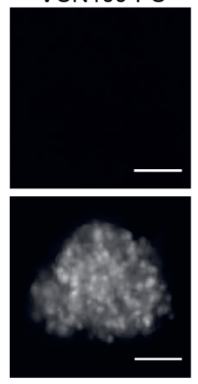

C

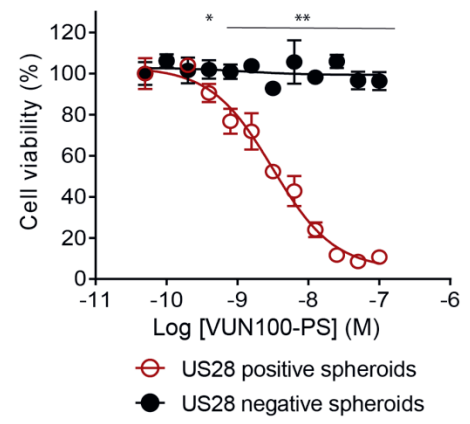

Figure 6. VUN100-PS selectively binds to US28-expressing spheroids and induces cell toxicity upon illumination. A) Staining of dead cells with propidium iodide (PI) and living cells (calcein) of US28 negative spheroids (US28 negative) and US28 positive spheroids (US28 positive). B) Incubation of VUN100-PS with US28 negative and positive spheroids. Spheroids and VUN100-PS were visualized with an EVOS microscope. C) Determination of cell viability after incubation with different concentrations of VUN100-PS and illumination with a $10 \mathrm{~J} / \mathrm{cm}^{2}$ light dose. Cell viability was determined using CellTiter-Glo ${ }^{\circledR} 3$ D reagent $(* \mathrm{p}<0.05 ; * * \mathrm{p}<0.01, \mathrm{t}$ test $)$.

\section{Discussion}

Multiple GPCRs, including chemokine receptors, are overexpressed in tumors including melanomas, breast, lung, colorectal, and head and neck cancer making them interesting targets for targeted therapies [26,46-50]. In this study, we set out to investigate the potential of GPCRs as targets for nanobody-targeted PDT by using the HCMV-encoded 
chemokine receptor US28 as a proof of concept. We developed a new US28 targeting nanobody (VUN100) with a superior binding affinity for US28, compared to our previously reported US28 nanobody [27]. Interestingly, different llamas, immunization procedures and distinct selection strategies resulted in the identification of a nanobody with high sequence similarity in the CDR3 region. Moreover, VUN100 bound the same epitope on US28, as the previously described US28 nanobody, although with a higher affinity. Because of the high CDR3 homology between these two different nanobodies, the increased affinity can be ascribed to differences in the frameworks and other CDR regions. This confirms the general notion that the CDR3 region plays a predominant role in determining the binding epitope of nanobodies, while affinity variations are more likely the consequence of variations in CDR and framework sequences [51-53]. The epitope of VUN100 involved both the N-terminus and ECL3 loop of US28. More specifically, although more residues in the N-terminus might be involved, the tyrosine at position 16 is important for binding of VUN100. In a previous study, this residue (and more specifically the sulfonyl group) was also found to be important for ligand binding, which correlates well with the observation that VUN100 displaces the known US28 ligands CCL5 and CX3CL1 [54]. Although VUN100 could displace multiple types of chemokine ligands from US28, we observed no nonspecific binding of VUN100 to CX3CR1, which is the chemokine receptor that shares the highest homology with US28, indicating the specificity of VUN100 for US28.

Our experiments show that nanobody-targeted PDT induced cell death of up to $90 \%$ of the US28 expressing cells. This percentage correlates with the percentage of cells with detectable US28 expression. However, it is currently unclear whether the cells that escaped PDT-mediated cell death are truly US28 negative or express the receptor at low levels. Using a stable cell line, it is likely that these presumably negative cells do express low levels of US28, though undetectable. The efficacy of the treatment could be enhanced further by increasing the amount of photosensitizer delivered to these cells. In contrast to a maximal degree of conjugation of 1 by directional conjugation, higher conjugation efficacies could potentially be achieved by random conjugation of photosensitizer to multiple lysines. However, conjugation to multiple lysines cannot be controlled easily and can also significantly harm the integrity of the nanobody (as was here observed for VUN100). Another way of increasing the delivery of photosensitizer is by intracellular delivery and residualization of the conjugate, such that the photosensitizer will accumulate 
in the cell. Depending on the chemical properties and size of fluorescent dyes, these molecules can residualize inside cells upon uptake. Using the endocytic machinery in cells, extended pulses with nanobody-photosensitizer conjugates could result in intracellular accumulation of photosensitizer which would favor PDT efficacy. Near-infrared dye IRDye800CW and the photosensitizer IRDye700DX are both known to residualize inside cells $[12,55]$. Previously, we have shown the additional PDT effect with an internalizing anti-EGFR nanobody-photosensitizer conjugate [12]. US28 is known to be constitutively internalized [56]. Potentially, this would allow repetitive uptake and accumulation of photosensitizer-conjugated nanobodies in US28-expressing cells, thereby enhancing PDT efficacy.

Antibodies have already shown to be good targeting moieties for targeted PDT [6,7]. However, their relatively large size, together with the binding site barrier hampers their tissue distribution [57]. Previous studies have already shown a faster and more homogenous distribution of nanobodies compared to antibodies $[13,58,59]$. Recently, the penetration of EGFR targeted nanobodies and the anti-EGFR monoclonal antibody cetuximab were assessed in $3 \mathrm{D}$ spheroids [60]. A clear delay in accumulation in the spheroid was seen for the monoclonal antibody compared to the nanobodies. In this study, selective killing of US28-positive glioblastoma cells was observed both in 2D and 3D cultures. Furthermore, no significant difference in efficacy was observed between PDT in $2 \mathrm{D}$ or $3 \mathrm{D}$ cultures. These results further substantiate the potential use of nanobodyphotosensitizer conjugates for GPCR-targeted PDT.

Although the blood-brain barrier is considered to be leaky in glioblastoma patients, different strategies have been described to enhance the crossing of nanobodies through the blood-brain barrier, including the modification of the isoelectric point of nanobodies [61-65]. Currently, conventional (untargeted) PDT is approved for intraoperative PDT of malignant brain tumors in Japan, making the application of nanobody-targeted PDT in the brain therefore conceivable $[66,67]$.

In this study, US28 was used as an example for GPCR-targeted PDT. Importantly, US28 expression is detected in various tumors, whereas it is only detected in a small percentage of latently infected myeloid cells in healthy individuals, making this receptor a very interesting (non-human) target for targeted therapies [32-38,68]. In addition, an US28targeting fusion toxin protein was able to kill latently infected myeloid cells, indicating the 
potential of US28-targeted therapies to eradicate HCMV-infected cells [69]. Although we are the first to describe GPCRs as targets for nanobody-targeted PDT, GPCR-targeted PDT has been reported previously for the type 2 Cannabinoid receptor $\left(\mathrm{CB}_{2} \mathrm{R}\right)[70]$. $\mathrm{A} \mathrm{CB}_{2} \mathrm{R}-$ targeting small molecule (mbc94) was conjugated to the photosensitizer IRDye700DX and killed around $80 \%$ of the $\mathrm{CB}_{2} \mathrm{R}$-overexpressing cells. However, this required micromolar concentrations of the conjugate, overnight incubation periods with conjugates and illumination with higher power density $\left(30 \mathrm{~mW} / \mathrm{cm}^{2}\right.$ and a total dose of $\left.36 \mathrm{~J} / \mathrm{cm}^{2}\right)$. With VUN100-PS, we were able to selectively induce cell toxicity in US28-positive glioblastoma cells after $1 \mathrm{~h}$ of incubation with conjugates, a power density of $5 \mathrm{~mW} / \mathrm{cm}^{2}$ and a total dose of $10 \mathrm{~J} / \mathrm{cm}^{2}$, resulting in nanomolar potency values. For these reasons, GPCR-targeting nanobodies have good potential for in vivo PDT.

To conclude, by using a novel US28-targeting nanobody-photosensitizer conjugate we selectively killed US28-expressing glioblastoma cells both in 2D and 3D cultures. This study shows the potential of GPCRs as targets for nanobody-directed PDT to treat proliferative diseases. In addition, US28-targeting nanobody-photosensitizer conjugates hold potential in treatment of HCMV associated malignancies.

\section{Acknowledgments}

This work was supported by the Netherlands Organization for Scientific Research (NWO: Vici grant 016.140.657) and the European Research Council (ERC) under the European Union's Horizon 2020 research and innovation program (grant agreement No 677582).

\section{References}

1. Agostinis, P.; Berg, K.; Cengel, K. A.; Foster, T. H.; Girotti, A. W.; Gollnick, S. O.; Hahn, S. M.; Hamblin, M. R.; Juzeniene, A.; Kessel, D.; Korbelik, M.; Moan, J.; Mroz, P.; Nowis, D.; Piette, J.; Wilson, B. C.; Golab, J. Photodynamic therapy of cancer: an update. CA Cancer J Clin 2011, 61, (4), 250-81.

2. Kwiatkowski, S.; Knap, B.; Przystupski, D.; Saczko, J.; Kedzierska, E.; Knap-Czop, K.; Kotlinska, J.; Michel, O.; Kotowski, K.; Kulbacka, J. Photodynamic therapy - mechanisms, photosensitizers and combinations. Biomed Pharmacother 2018, 106, 1098-1107.

3. Calixto, G. M.; Bernegossi, J.; de Freitas, L. M.; Fontana, C. R.; Chorilli, M. NanotechnologyBased Drug Delivery Systems for Photodynamic Therapy of Cancer: A Review. Molecules 2016, 21, (3), 342.

4. Martinez De Pinillos Bayona, A.; Mroz, P.; Thunshelle, C.; Hamblin, M. R. Design features for optimization of tetrapyrrole macrocycles as antimicrobial and anticancer photosensitizers. Chem Biol Drug Des 2017, 89, (2), 192-206.

5. Gomer, C. J. Preclinical examination of first and second generation photosensitizers used in 
photodynamic therapy. Photochem Photobiol 1991, 54, (6), 1093-107.

6. van Dongen, G. A.; Visser, G. W.; Vrouenraets, M. B. Photosensitizer-antibody conjugates for detection and therapy of cancer. Adv Drug Deliv Rev 2004, 56, (1), 31-52.

7. Mitsunaga, M.; Ogawa, M.; Kosaka, N.; Rosenblum, L. T.; Choyke, P. L.; Kobayashi, H. Cancer cell-selective in vivo near infrared photoimmunotherapy targeting specific membrane molecules. Nat Med 2011, 17, (12), 1685-91.

8. van Straten, D.; Mashayekhi, V.; de Bruijn, H. S.; Oliveira, S.; Robinson, D. J. Oncologic Photodynamic Therapy: Basic Principles, Current Clinical Status and Future Directions. Cancers (Basel) 2017, 9, (2).

9. Beckman, R. A.; Weiner, L. M.; Davis, H. M. Antibody constructs in cancer therapy: protein engineering strategies to improve exposure in solid tumors. Cancer 2007, 109, (2), 170-9.

10. Thurber, G. M.; Zajic, S. C.; Wittrup, K. D. Theoretic criteria for antibody penetration into solid tumors and micrometastases. J Nucl Med 2007, 48, (6), 995-9.

11. Watanabe, R.; Hanaoka, H.; Sato, K.; Nagaya, T.; Harada, T.; Mitsunaga, M.; Kim, I.; Paik, C. H.; Wu, A. M.; Choyke, P. L.; Kobayashi, H. Photoimmunotherapy targeting prostate-specific membrane antigen: are antibody fragments as effective as antibodies? J Nucl Med 2015, 56, (1), $140-4$.

12. Heukers, R.; van Bergen en Henegouwen, P. M.; Oliveira, S. Nanobody-photosensitizer conjugates for targeted photodynamic therapy. Nanomedicine 2014, 10, (7), 1441-51.

13. van Driel, P.; Boonstra, M. C.; Slooter, M. D.; Heukers, R.; Stammes, M. A.; Snoeks, T. J. A.; de Bruijn, H. S.; van Diest, P. J.; Vahrmeijer, A. L.; van Bergen En Henegouwen, P. M. P.; van de Velde, C. J. H.; Lowik, C.; Robinson, D. J.; Oliveira, S. EGFR targeted nanobodyphotosensitizer conjugates for photodynamic therapy in a pre-clinical model of head and neck cancer. J Control Release 2016, 229, 93-105.

14. Muyldermans, S.; Baral, T. N.; Retamozzo, V. C.; De Baetselier, P.; De Genst, E.; Kinne, J.; Leonhardt, H.; Magez, S.; Nguyen, V. K.; Revets, H.; Rothbauer, U.; Stijlemans, B.; Tillib, S.; Wernery, U.; Wyns, L.; Hassanzadeh-Ghassabeh, G.; Saerens, D. Camelid immunoglobulins and nanobody technology. Veterinary immunology and immunopathology 2009, 128, (1-3), 17883.

15. Harmsen, M. M.; De Haard, H. J. Properties, production, and applications of camelid singledomain antibody fragments. Appl Microbiol Biotechnol 2007, 77, (1), 13-22.

16. De Genst, E.; Silence, K.; Decanniere, K.; Conrath, K.; Loris, R.; Kinne, J.; Muyldermans, S.; Wyns, L. Molecular basis for the preferential cleft recognition by dromedary heavy-chain antibodies. Proc Natl Acad Sci U S A 2006, 103, (12), 4586-91.

17. De Groof, T. W. M.; Bobkov, V.; Heukers, R.; Smit, M. J. Nanobodies: New avenues for imaging, stabilizing and modulating GPCRs. Mol Cell Endocrinol 2019, 484, 15-24.

18. Rosenbaum, D. M.; Rasmussen, S. G.; Kobilka, B. K. The structure and function of G-proteincoupled receptors. Nature 2009, 459, (7245), 356-63.

19. Kobilka, B. The structural basis of G-protein-coupled receptor signaling (Nobel Lecture). Angew Chem Int Ed Engl 2013, 52, (25), 6380-8.

20. Bar-Shavit, R.; Maoz, M.; Kancharla, A.; Nag, J. K.; Agranovich, D.; Grisaru-Granovsky, S.; Uziely, B. G Protein-Coupled Receptors in Cancer. Int J Mol Sci 2016, 17, (8).

21. Dorsam, R. T.; Gutkind, J. S. G-protein-coupled receptors and cancer. Nat Rev Cancer 2007, 7, (2), 79-94.

22. O'Hayre, M.; Degese, M. S.; Gutkind, J. S. Novel insights into G protein and G protein-coupled receptor signaling in cancer. Curr Opin Cell Biol 2014, 27, 126-35.

23. O'Hayre, M.; Vazquez-Prado, J.; Kufareva, I.; Stawiski, E. W.; Handel, T. M.; Seshagiri, S.; Gutkind, J. S. The emerging mutational landscape of $\mathrm{G}$ proteins and G-protein-coupled receptors in cancer. Nat Rev Cancer 2013, 13, (6), 412-24.

24. Jahnichen, S.; Blanchetot, C.; Maussang, D.; Gonzalez-Pajuelo, M.; Chow, K. Y.; Bosch, L.; De Vrieze, S.; Serruys, B.; Ulrichts, H.; Vandevelde, W.; Saunders, M.; De Haard, H. J.; Schols, D.; Leurs, R.; Vanlandschoot, P.; Verrips, T.; Smit, M. J. CXCR4 nanobodies (VHH-based single variable domains) potently inhibit chemotaxis and HIV-1 replication and mobilize stem cells. Proc Natl Acad Sci U S A 2010, 107, (47), 20565-70. 
25. de Wit, R. H.; Heukers, R.; Brink, H. J.; Arsova, A.; Maussang, D.; Cutolo, P.; Strubbe, B.; Vischer, H. F.; Bachelerie, F.; Smit, M. J. CXCR4-Specific Nanobodies as Potential Therapeutics for WHIM syndrome. J Pharmacol Exp Ther 2017, 363, (1), 35-44.

26. Maussang, D.; Mujic-Delic, A.; Descamps, F. J.; Stortelers, C.; Vanlandschoot, P.; Stigter-van Walsum, M.; Vischer, H. F.; van Roy, M.; Vosjan, M.; Gonzalez-Pajuelo, M.; van Dongen, G. A.; Merchiers, P.; van Rompaey, P.; Smit, M. J. Llama-derived single variable domains (nanobodies) directed against chemokine receptor CXCR7 reduce head and neck cancer cell growth in vivo. J Biol Chem 2013, 288, (41), 29562-72.

27. Heukers, R.; Fan, T. S.; de Wit, R. H.; van Senten, J. R.; De Groof, T. W. M.; Bebelman, M. P.; Lagerweij, T.; Vieira, J.; de Munnik, S. M.; Smits-de Vries, L.; van Offenbeek, J.; Rahbar, A.; van Hoorick, D.; Soderberg-Naucler, C.; Wurdinger, T.; Leurs, R.; Siderius, M.; Vischer, H. F.; Smit, M. J. The constitutive activity of the virally encoded chemokine receptor US28 accelerates glioblastoma growth. Oncogene 2018, 37, (30), 4110-4121.

28. Peyrassol, X.; Laeremans, T.; Gouwy, M.; Lahura, V.; Debulpaep, M.; Van Damme, J.; Steyaert, J.; Parmentier, M.; Langer, I. Development by Genetic Immunization of Monovalent Antibodies (Nanobodies) Behaving as Antagonists of the Human ChemR23 Receptor. J Immunol 2016, 196 , (6), 2893-901.

29. Scholler, P.; Nevoltris, D.; de Bundel, D.; Bossi, S.; Moreno-Delgado, D.; Rovira, X.; Moller, T. C.; El Moustaine, D.; Mathieu, M.; Blanc, E.; McLean, H.; Dupuis, E.; Mathis, G.; Trinquet, E.; Daniel, H.; Valjent, E.; Baty, D.; Chames, P.; Rondard, P.; Pin, J. P. Allosteric nanobodies uncover a role of hippocampal mGlu2 receptor homodimers in contextual fear consolidation. Nat Commun 2017, 8, (1), 1967.

30. Gandhi, M. K.; Khanna, R. Human cytomegalovirus: clinical aspects, immune regulation, and emerging treatments. The Lancet Infectious Diseases 2004, 4, (12), 725-738.

31. Vischer, H. F.; Siderius, M.; Leurs, R.; Smit, M. J. Herpesvirus-encoded GPCRs: neglected players in inflammatory and proliferative diseases? Nat Rev Drug Discov 2014, 13, (2), 123-39.

32. Soroceanu, L.; Matlaf, L.; Bezrookove, V.; Harkins, L.; Martinez, R.; Greene, M.; Soteropoulos, P.; Cobbs, C. S. Human cytomegalovirus US28 found in glioblastoma promotes an invasive and angiogenic phenotype. Cancer Res 2011, 71, (21), 6643-53.

33. Dziurzynski, K.; Chang, S. M.; Heimberger, A. B.; Kalejta, R. F.; McGregor Dallas, S. R.; Smit, M.; Soroceanu, L.; Cobbs, C. S.; Hcmv; Gliomas, S. Consensus on the role of human cytomegalovirus in glioblastoma. Neuro Oncol 2012, 14, (3), 246-55.

34. Harkins, L.; Volk, A. L.; Samanta, M.; Mikolaenko, I.; Britt, W. J.; Bland, K. I.; Cobbs, C. S. Specific localisation of human cytomegalovirus nucleic acids and proteins in human colorectal cancer. Lancet 2002, 360, (9345), 1557-63.

35. Samanta, M.; Harkins, L.; Klemm, K.; Britt, W. J.; Cobbs, C. S. High prevalence of human cytomegalovirus in prostatic intraepithelial neoplasia and prostatic carcinoma. J Urol 2003, 170, (3), 998-1002.

36. Mitchell, D. A.; Xie, W.; Schmittling, R.; Learn, C.; Friedman, A.; McLendon, R. E.; Sampson, J. H. Sensitive detection of human cytomegalovirus in tumors and peripheral blood of patients diagnosed with glioblastoma. Neuro Oncol 2008, 10, (1), 10-8.

37. Cobbs, C. S.; Harkins, L.; Samanta, M.; Gillespie, G. Y.; Bharara, S.; King, P. H.; Nabors, L. B.; Cobbs, C. G.; Britt, W. J. Human cytomegalovirus infection and expression in human malignant glioma. Cancer Res 2002, 62, (12), 3347-50.

38. Scheurer, M. E.; Bondy, M. L.; Aldape, K. D.; Albrecht, T.; El-Zein, R. Detection of human cytomegalovirus in different histological types of gliomas. Acta Neuropathol 2008, 116, (1), 7986.

39. Maussang, D.; Langemeijer, E.; Fitzsimons, C. P.; Stigter-van Walsum, M.; Dijkman, R.; Borg, M. K.; Slinger, E.; Schreiber, A.; Michel, D.; Tensen, C. P.; van Dongen, G. A.; Leurs, R.; Smit, M. J. The human cytomegalovirus-encoded chemokine receptor US28 promotes angiogenesis and tumor formation via cyclooxygenase-2. Cancer Res 2009, 69, (7), 2861-9.

40. Maussang, D.; Verzijl, D.; van Walsum, M.; Leurs, R.; Holl, J.; Pleskoff, O.; Michel, D.; van Dongen, G. A.; Smit, M. J. Human cytomegalovirus-encoded chemokine receptor US28 promotes tumorigenesis. Proc Natl Acad Sci U S A 2006, 103, (35), 13068-73. 
41. Slinger, E.; Maussang, D.; Schreiber, A.; Siderius, M.; Rahbar, A.; Fraile-Ramos, A.; Lira, S A.; Soderberg-Naucler, C.; Smit, M. J. HCMV-encoded chemokine receptor US28 mediates proliferative signaling through the IL-6-STAT3 axis. Sci Signal 2010, 3, (133), ra58.

42. Kooijmans, S. A.; Aleza, C. G.; Roffler, S. R.; van Solinge, W. W.; Vader, P.; Schiffelers, R. M. Display of GPI-anchored anti-EGFR nanobodies on extracellular vesicles promotes tumour cell targeting. J Extracell Vesicles 2016, 5, 31053.

43. Goldman, L. A.; Cutrone, E. C.; Kotenko, S. V.; Krause, C. D.; Langer, J. A. Modifications of vectors pEF-BOS, pcDNA1 and pcDNA3 result in improved convenience and expression. Biotechniques 1996, 21, (6), 1013-5.

44. Casarosa, P.; Menge, W. M.; Minisini, R.; Otto, C.; van Heteren, J.; Jongejan, A.; Timmerman, H.; Moepps, B.; Kirchhoff, F.; Mertens, T.; Smit, M. J.; Leurs, R. Identification of the first nonpeptidergic inverse agonist for a constitutively active viral-encoded $\mathrm{G}$ protein-coupled receptor. J Biol Chem 2003, 278, (7), 5172-8.

45. de Wit, R. H.; Mujic-Delic, A.; van Senten, J. R.; Fraile-Ramos, A.; Siderius, M.; Smit, M. J. Human cytomegalovirus encoded chemokine receptor US28 activates the HIF-1alpha/PKM2 axis in glioblastoma cells. Oncotarget 2016, 7, (42), 67966-67985.

46. Kim, J.; Mori, T.; Chen, S. L.; Amersi, F. F.; Martinez, S. R.; Kuo, C.; Turner, R. R.; Ye, X.; Bilchik, A. J.; Morton, D. L.; Hoon, D. S. Chemokine receptor CXCR4 expression in patients with melanoma and colorectal cancer liver metastases and the association with disease outcome. Ann Surg 2006, 244, (1), 113-20.

47. Mukherjee, D.; Zhao, J. The Role of chemokine receptor CXCR4 in breast cancer metastasis. Am J Cancer Res 2013, 3, (1), 46-57.

48. Kim, J.; Takeuchi, H.; Lam, S. T.; Turner, R. R.; Wang, H. J.; Kuo, C.; Foshag, L.; Bilchik, A. J.; Hoon, D. S. Chemokine receptor CXCR4 expression in colorectal cancer patients increases the risk for recurrence and for poor survival. J Clin Oncol 2005, 23, (12), 2744-53.

49. Miao, Z.; Luker, K. E.; Summers, B. C.; Berahovich, R.; Bhojani, M. S.; Rehemtulla, A.; Kleer, C. G.; Essner, J. J.; Nasevicius, A.; Luker, G. D.; Howard, M. C.; Schall, T. J. CXCR7 (RDC1) promotes breast and lung tumor growth in vivo and is expressed on tumor-associated vasculature. Proc Natl Acad Sci U S A 2007, 104, (40), 15735-40.

50. Hattermann, K.; Held-Feindt, J.; Lucius, R.; Muerkoster, S. S.; Penfold, M. E.; Schall, T. J.; Mentlein, R. The chemokine receptor CXCR7 is highly expressed in human glioma cells and mediates antiapoptotic effects. Cancer Res 2010, 70, (8), 3299-308.

51. Bannas, P.; Hambach, J.; Koch-Nolte, F. Nanobodies and Nanobody-Based Human Heavy Chain Antibodies As Antitumor Therapeutics. Front Immunol 2017, 8, 1603.

52. Muyldermans, S. Nanobodies: natural single-domain antibodies. Annu Rev Biochem 2013, 82, 775-97.

53. Zavrtanik, U.; Lukan, J.; Loris, R.; Lah, J.; Hadzi, S. Structural Basis of Epitope Recognition by Heavy-Chain Camelid Antibodies. J Mol Biol 2018, 430, (21), 4369-4386.

54. Casarosa, P.; Waldhoer, M.; LiWang, P. J.; Vischer, H. F.; Kledal, T.; Timmerman, H.; Schwartz, T. W.; Smit, M. J.; Leurs, R. CC and CX3C chemokines differentially interact with the $\mathrm{N}$ terminus of the human cytomegalovirus-encoded US28 receptor. J Biol Chem 2005, 280, (5), 3275-85.

55. Cilliers, C.; Liao, J.; Atangcho, L.; Thurber, G. M. Residualization Rates of Near-Infrared Dyes for the Rational Design of Molecular Imaging Agents. Mol Imaging Biol 2015, 17, (6), 757-62.

56. Fraile-Ramos, A.; Kledal, T. N.; Pelchen-Matthews, A.; Bowers, K.; Schwartz, T. W.; Marsh, M. The human cytomegalovirus US28 protein is located in endocytic vesicles and undergoes constitutive endocytosis and recycling. Mol Biol Cell 2001, 12, (6), 1737-49.

57. Juweid, M.; Neumann, R.; Paik, C.; Perez-Bacete, M. J.; Sato, J.; van Osdol, W.; Weinstein, J. $\mathrm{N}$. Micropharmacology of monoclonal antibodies in solid tumors: direct experimental evidence for a binding site barrier. Cancer Res 1992, 52, (19), 5144-53.

58. Oliveira, S.; van Dongen, G. A.; Stigter-van Walsum, M.; Roovers, R. C.; Stam, J. C.; Mali, W.; van Diest, P. J.; van Bergen en Henegouwen, P. M. Rapid visualization of human tumor xenografts through optical imaging with a near-infrared fluorescent anti-epidermal growth factor receptor nanobody. Mol Imaging 2012, 11, (1), 33-46. 
59. Kijanka, M.; Warnders, F. J.; El Khattabi, M.; Lub-de Hooge, M.; van Dam, G. M.; Ntziachristos, V.; de Vries, L.; Oliveira, S.; van Bergen En Henegouwen, P. M. Rapid optical imaging of human breast tumour xenografts using anti-HER2 VHHs site-directly conjugated to IRDye 800CW for image-guided surgery. Eur J Nucl Med Mol Imaging 2013, 40, (11), 1718-29.

60. Beltran Hernandez, I.; Rompen, R.; Rossin, R.; Xenaki, K. T.; Katrukha, E. A.; Nicolay, K.; van Bergen En Henegouwen, P.; Grull, H.; Oliveira, S. Imaging of Tumor Spheroids, Dual-Isotope SPECT, and Autoradiographic Analysis to Assess the Tumor Uptake and Distribution of Different Nanobodies. Mol Imaging Biol 2019.

61. van Tellingen, O.; Yetkin-Arik, B.; de Gooijer, M. C.; Wesseling, P.; Wurdinger, T.; de Vries, H. E. Overcoming the blood-brain tumor barrier for effective glioblastoma treatment. Drug Resist Updat 2015, 19, 1-12.

62. Rissiek, B.; Koch-Nolte, F.; Magnus, T. Nanobodies as modulators of inflammation: potential applications for acute brain injury. Front Cell Neurosci 2014, 8, 344

63. Li, T.; Bourgeois, J. P.; Celli, S.; Glacial, F.; Le Sourd, A. M.; Mecheri, S.; Weksler, B.; Romero, I.; Couraud, P. O.; Rougeon, F.; Lafaye, P. Cell-penetrating anti-GFAP VHH and corresponding fluorescent fusion protein VHH-GFP spontaneously cross the blood-brain barrier and specifically recognize astrocytes: application to brain imaging. FASEB $J \mathbf{2 0 1 2}, 26$, (10), 3969-79.

64. Li, T.; Vandesquille, M.; Koukouli, F.; Dudeffant, C.; Youssef, I.; Lenormand, P.; Ganneau, C.; Maskos, U.; Czech, C.; Grueninger, F.; Duyckaerts, C.; Dhenain, M.; Bay, S.; Delatour, B.; Lafaye, P. Camelid single-domain antibodies: A versatile tool for in vivo imaging of extracellular and intracellular brain targets. $J$ Control Release 2016, 243, 1-10.

65. Bruce, V. J.; Lopez-Islas, M.; McNaughton, B. R. Resurfaced cell-penetrating nanobodies: A potentially general scaffold for intracellularly targeted protein discovery. Protein Sci 2016, 25, (6), 1129-37.

66. Quirk, B. J.; Brandal, G.; Donlon, S.; Vera, J. C.; Mang, T. S.; Foy, A. B.; Lew, S. M.; Girotti, A. W.; Jogal, S.; LaViolette, P. S.; Connelly, J. M.; Whelan, H. T. Photodynamic therapy (PDT) for malignant brain tumors--where do we stand? Photodiagnosis Photodyn Ther 2015, 12, (3), $530-44$.

67. Akimoto, J. Photodynamic Therapy for Malignant Brain Tumors. Neurol Med Chir (Tokyo) 2016, 56, (4), 151-7.

68. Slobedman, B.; Mocarski, E. S. Quantitative analysis of latent human cytomegalovirus. J Virol 1999, 73, (6), 4806-12.

69. Krishna, B. A.; Spiess, K.; Poole, E. L.; Lau, B.; Voigt, S.; Kledal, T. N.; Rosenkilde, M. M.; Sinclair, J. H. Targeting the latent cytomegalovirus reservoir with an antiviral fusion toxin protein. Nat Commun 2017, 8, 14321.

70. Zhang, S.; Jia, N.; Shao, P.; Tong, Q.; Xie, X. Q.; Bai, M. Target-selective phototherapy using a ligand-based photosensitizer for type 2 cannabinoid receptor. Chem Biol 2014, 21, (3), 338-44. 


\section{Supplementary Data}

Figure S1

A

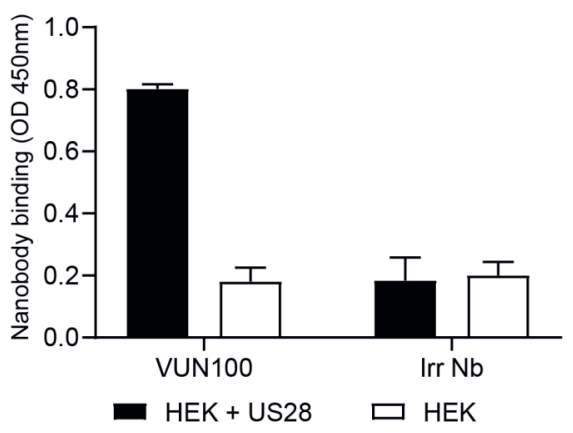

B

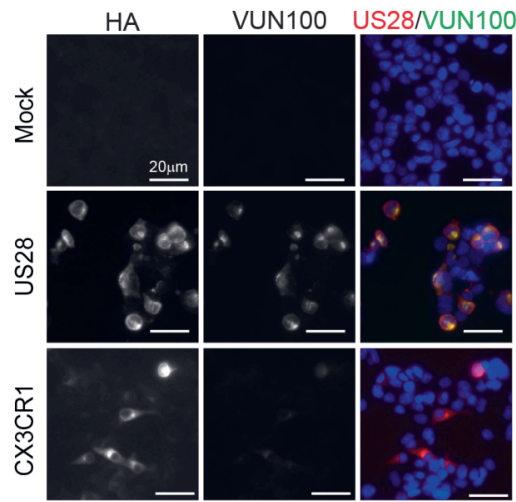

Figure S1. VUN100 binds selectively to US28. A) Binding of VUN100 and irrelevant nanobody (binding to the azodye RR6; Irr NB) to US28-expressing HEK293T membranes (HEK+US28) and mock transfected HEK293T membranes (HEK), as determined by ELISA. B) Immunofluorescence microscopy of the binding of VUN100 to US28 and CX3CR1. Receptor expression was detected using an N-terminal HA-tag and anti-HA antibody (HA). VUN100 binding was detected using the Myc-tag and an anti-Myc antibody (VUN100). 
Figure S2

A

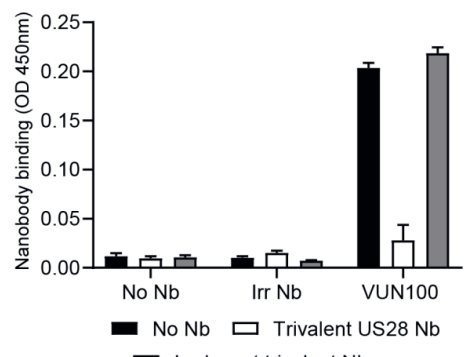

$\square$ Irrelevant trivalent $\mathrm{Nb}$
$\mathrm{B}$

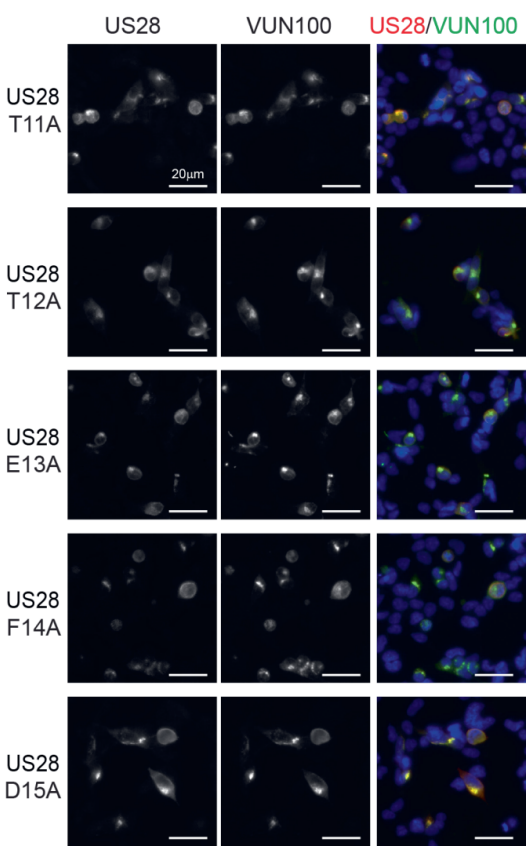

Figure S2. VUN100 binds the same epitope as the US28 NB. A) Competition binding ELISA of VUN100 and irrelevant nanobody (binding to the azodye RR6; Irr NB) to US28-expressing HEK293T membranes (HEK+US28). Binding of the nanobodies was detected using the Myc-tag and an anti-Myc antibody. Nanobodies were displaced by untagged trivalent US28 nanobody (US28 NB) or untagged trivalent irrelevant nanobody. B) Immunofluorescence microscopy of the binding of VUN100 to N-terminus US28 mutants with the amino acids at positions 11-15 being substituted by alanines. 


\section{Figure S3}

A

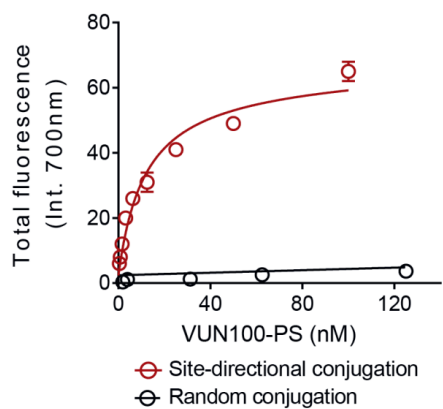

B

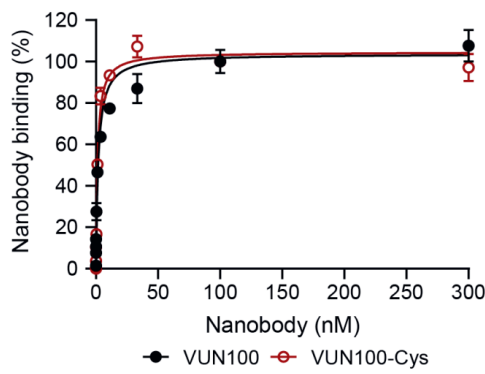

Figure S3. Binding of randomly conjugated VUN100-PS and VUN100-Cys to US28. A) Binding of different concentrations of randomly or site-directionally conjugated VUN100-PS to US28 positive cells on ice. Fluorescence of VUN100-PS bound to cells was detected using an Odyssey infrared scanner at $700 \mathrm{~nm}$. B) Binding of different concentrations of VUN100 and VUN100-Cys to US28expressing membranes. Specific binding is shown after subtraction of aspecific binding to US28negative membranes.

\section{Figure S4}

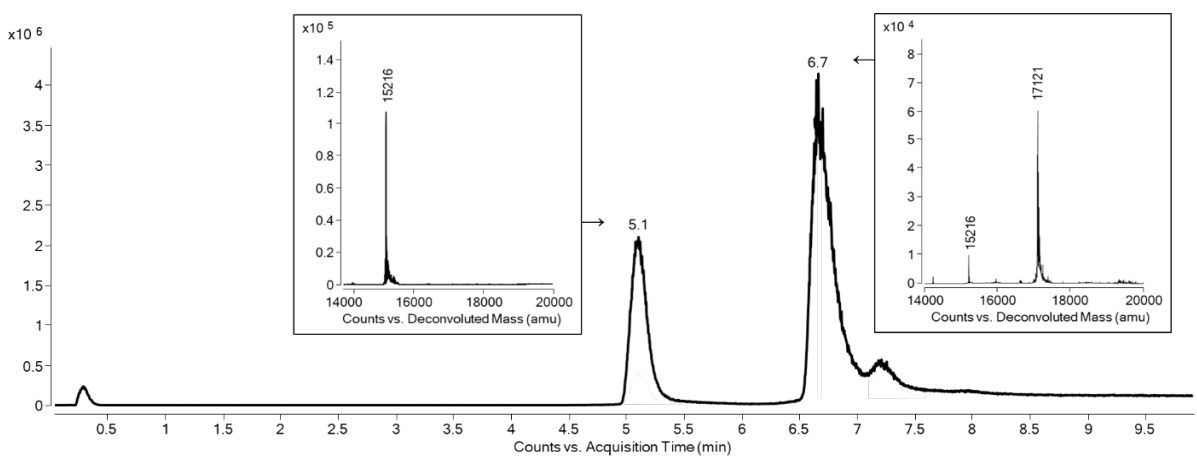

Figure S4. Extracted biomolecule LC-MS chromatogram of VUN100-PS. Photosensitizer conjugated $(6.7 \mathrm{~min})$ and unconjugated nanobodies $(5.1 \mathrm{~min})$ are separated and identified according to their deconvoluted mass spectra (inserts). 


\section{Figure S5}

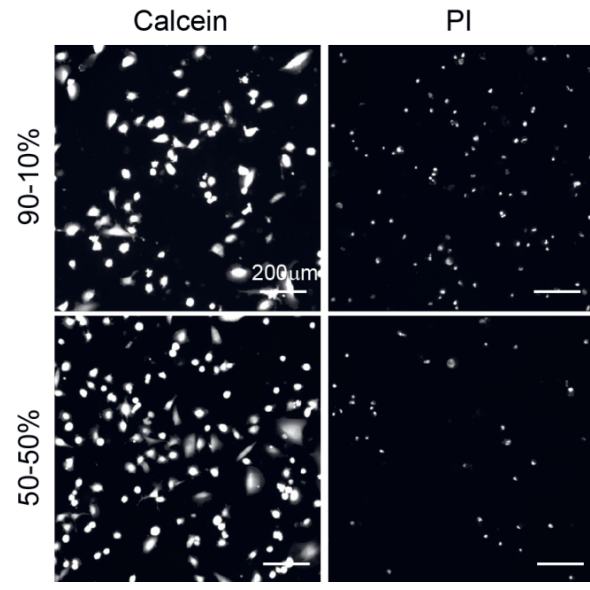

\section{VUN100-PS Calcein/PINUN100-PS}

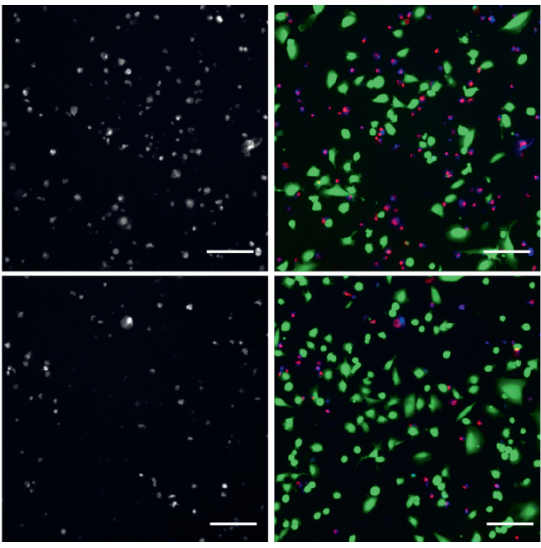

Figure S5. Nanobody-targeted PDT treatment on different ratios of co cultures of US28 positive and US28 negative cells. Different ratios of US28 positive and US28 negative cells were co-cultured (90$10 \%$ and $50-50 \%$ of US28 positive and US28 negative cells). Cells were stained with propidium iodide (PI) and calcein) $24 \mathrm{~h}$ after nanobody-targeted PDT to determine cell viability and the selectivity of the nanobody-targeted PDT. 


\section{Chapter 3}

$\mathrm{VHH}$-photosensitizer conjugates for targeted photodynamic therapy of Met-overexpressing tumor cells

Raimond Heukers, Vida Mashayekhi, Mercedes Ramirez-Escudero, Hans de Haard, Theo C. Verrips, Paul. M.P. van Bergen en Henegouwen, and Sabrina Oliveira

Antibodies, 2019; 8(2): 26

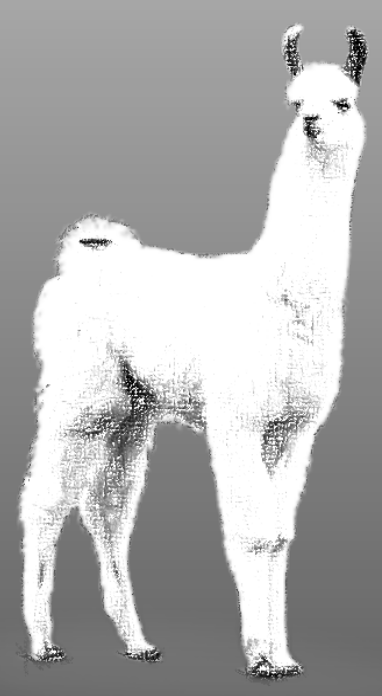




\section{ABSTRACT}

Photodynamic therapy (PDT) is an approach that kills (cancer) cells by the local production of toxic reactive oxygen species, upon the local illumination of a photosensitizer (PS). The specificity of PDT has been further enhanced by the development of new water-soluble PS and by the specific delivery of PS via conjugation to tumor-targeting antibodies. To improve tissue penetration and shorten photosensitivity, we have recently introduced nanobodies, also known as VHH (variable domains from the heavy chain of llama heavy chain antibodies), for targeted PDT of cancer cells overexpressing the epidermal growth factor receptor (EGFR). Overexpression and activation of another cancer-related receptor, the hepatocyte growth factor receptor (HGFR, c-Met or Met) is also involved in the progression and metastasis of a large variety of malignanices. Here, we have evaluated whether anti-Met VHHs conjugated to PS can also serve as a biopharmaceutical for targeted PDT. VHHs targeting the SEMA subdomain of Met were provided with a Cterminal tag that allowed both straightforward purification from yeast supernatant and directional conjugation to the PS IRDye700DX using maleimide chemistry. The generated anti-Met VHH-PS showed nanomolar binding affinity and, upon illumination, specifically killed MKN45 cells with nanomolar potency. This study shows that Met can also serve as a membrane target for targeted PDT. 


\section{Introduction}

Photodynamic therapy (PDT) is a type of cancer treatment in which tumor cells are killed by reactive oxygen species, such as singlet oxygen, formed by the local and lightinduced activation of a photosensitizer (PS) [1]. By locally reacting with proteins, lipids and nucleic acids, the reactive oxygen species generated can hereby induce cell death, vascular damage, and an inflammatory response [2]. It is for this mode-of-action that PDT is used in the clinic to treat malignancies. Unfortunately, the PS generally used in the clinic are relatively hydrophobic, are systemically applied, and are non-targeted. These factors combined can result in off-target toxicity and long lasting photosensitivity [2,3]. Improved strategies involve the development of more water soluble PS, delivery of PS with nanocarriers and photo-immunotherapy (PIT), among others [4-6]. PIT is one of the strategies of targeted PDT, in which the PS is delivered selectively to tumors via conjugation to tumor-targeting antibodies.

In order to increase the efficacy of targeted PDT and reduce the period of photosensitivity, we have recently introduced smaller PS-conjugates with enhanced tumor penetration in combination with reduced blood circulation time $[7,8]$. These smaller conjugates were generated by conjugating a water soluble PS to small antibody fragments, i.e. nanobodies or VHHs, of which the latter stands for the variable domain of the heavy chain from heavy chain-only antibodies found in animals of the Camelidae family [9]. Compared to commonly used conventional antibodies of the IgG class, VHHs are ten times smaller and consist of only a single domain with in general only one, rarely two disulfide bridges $[10,11]$. These features favour the selection and production process and make them very stable [11]. Also, because the C-terminus of a VHH is located opposite to its epitopebinding loops (i.e. complementary determining regions), C-terminal conjugation to effector molecules generally does not affect the binding properties [12-16].

Previously, we have described the nanobody-PS or VHH-PS conjugates specifically targeting the epidermal growth factor receptor (EGFR), a receptor tyrosine kinase which is found overexpressed on a large variety of cancers, such as head and neck, lung or colon cancer [17,18]. In vitro, upon illumination, the VHH-PS conjugates selectively killed EGFR overexpressing tumor cells with nanomolar $\mathrm{EC}_{50}$ values. In vivo, VHH-targeted PDT induced 80-90\% tumor necrosis, as measured $24 \mathrm{~h}$ after illumination $[7,8]$. Another receptor tyrosine kinase, which is frequently overexpressed or deregulated in 
a large number of carcinomas, sarcomas, haematopoietic malignancies and other neoplasms is the hepatocyte growth factor receptor (HGFR, c-Met or Met) [19,20]. Met is a $195 \mathrm{kDa}$ hetero-dimeric single membrane spanning receptor tyrosine kinase that is activated by hepatocyte growth factor (HGF, also known as scatter factor) [21]. The extracellular part of Met consists of three subdomains: an N-terminal 7-bladed $\beta$-propeller-like SEMA (semaphorin) subdomain, a PSI (plexin, semaphorin, integrin-like) subdomain and four IPT (immuno-globulin-like) subdomains [22,23]. Blades 2-3 of the SEMA subdomain and IPT 3-4 interact with its natural ligand HGF [24]. In a previous study, multiple VHHs targeting the extracellular part of Met were developed (i.e. E9, F5 and G2) [25]. Of these VHHs, G2 was used to target crosslinked albumin nanoparticles to Met-expressing cells [25]. By using the anti-Met VHH G2, the targeted nanoparticles showed specific binding and uptake into Met-expressing cells.

In this study, we have characterized these Met targeting VHHs further by assessing to which subdomain they bind. Moreover, we have used the best of these VHHs for the specific delivery of water soluble PS to Met-expressing cells for targeted PDT. That VHH was extended with a C-terminal C-Direct tag that allows for affinity purification from yeast supernatant and for directional conjugation of the PS to an unpaired cysteine, using maleimide chemistry. Subsequently, binding of this conjugate to cells and their ability to kill Met-expressing cells by PDT were evaluated.

\section{Experimental section}

\section{Molecular modeling}

The molecular structure of G2c with the C-Direct tag was modelled with I-TASSER [26] and vizualized using the PyMOL Molecular Graphics System, Version 2.0 Schrödinger, LLC. NB10 from PDB ID 4DKA:A showed 85\% homology to G2 and was taken as a reference [27].

\section{VHH production and purification}

Selection and characterization of VHH clones targeting Met have been described previously [25]. VHH protein for epitope mapping was obtained from production in E. coli TG1. For that the VHH genes were clones in E. coli production vector pMEK222 containing a C-terminal FLAG-His tag. VHHs were produced and purified from E. coli TG1 by immobilized metal affinity chromatography (IMAC, Thermo Fisher Scientific, 
Waltham, Massachusetts, USA) as previously described [7,28]. For production in yeast, VHH genes were recloned in the pYQVQ11 yeast production plasmid harboring a C-terminal C-Direct tag containing a free thiol (cysteine) and an EPEA purification tag (C-tag, Thermo Fisher Scientific). To improve production yields and facilitate purification from supernatant, C-Direct-tagged VHH were produced in S. cerevisiae strain VWK18 as described previously [28-31]. VHHs were purified from the yeast supernatant using an Äkta Start (GE Healthcare, Chicago, Illinois, USA), a Capture-Select affinity chromatography column (Thermo Fisher Scientific) and size exclusion chromatography (Thermo Fisher Scientific) according to the manufacturers protocols. Purified VHH was filter sterilized and stored in PBS.

\section{Cell culture}

Met-overexpressing human gastric cancer cell line MKN45 (ACC-409) was obtained from the Deutsche Sammlung von Mikroorganismen und Zellkulturen (DSMZ, Braunschweig, Germany). The human ovarian carcinoma cell line TOV112D (CRL-11731) was obtained from American Tissue Culture Collection (ATCC, LGC Standards GmbH, Wesel, Germany). TOV112D cells that stably express Met (TOV+Met) were previously described [25]. These cell lines were cultured as previously described in either DMEM, (Thermo Fisher Scientific) for TOV112D cells or RPMI 1640 (Thermo Fisher Scientific) for MKN45 cells [25]. HepG2 cells were obtained from ATCC (LGC Standards GmbH) and were cultured in DMEM low glucose (Thermo Fisher Scientific). All media was supplemented with streptomycin, penicillin, l-glutamine and FCS as described previously [25].

\section{VHH binding ELISA}

Binding affinity and epitopes were determined by ELISA on Met ectodomain. Human Met, llama Met, the llama/human chimeric Met ectodomains and the control antibody c224G11 were described previously [32]. Maxisorp 96-wells plates (Thermo Fisher Scientific) were coated overnight with $100 \mu \mathrm{l}$ of $2 \mu \mathrm{g} / \mathrm{ml}$ of ectodomain and subsequently blocked with 4\% milk in PBS. Bound c224G11 was detected with donkeyanti-mouse-HRP (Bio-Rad, Hercules, California, USA) and bound VHH was detected using mouse-anti-FLAG (clone M2, Sigma-Aldrich, St. Louis, Missouri, USA) followed by donkey-anti-mouse-HRP (Bio-Rad) and TMB Ultra as substrate (Thermo Fisher Scientific). Antibody incubations were performed in $1 \%$ milk in PBS and for $1 \mathrm{~h}$ at room 
temperature. Absorbance was measured using a Multiskan Go plate reader (Thermo Fisher Scientific). Binding of PS-conjugated VHH was carried out in cells in binding medium (DMEM without phenol red, 50 mM HEPES pH 7.5, supplemented with $1 \%$ bovine serum albumin) for $2 \mathrm{~h}$ at $4{ }^{\circ} \mathrm{C}$ to prevent internalization. Bound VHH-PS was detected using the Odyssey near-infrared scanner (LI-COR Biosciences, Lincoln, Nebraska, USA). Data was plotted and fitted for one-site-specific binding using Prism 7 software (GraphPad, La Jolla, California, USA).

\section{Surface plasmon resonance (SPR)}

Met ectodomain variants (human, llama, chimera LS1 and LS5) were aminecoupled on a pre-activated G-COOH sensor chip (SensEye) with a Continuous Flow Microspotter (CFM, Wasatch Microfluidics) at $\mathrm{pH} 4.5$ (50 mM Sodium acetate buffer, $0.005 \%$ Tween 20). Beforehand, the sensor chip was equilibrated in $50 \mathrm{mM}$ MES buffer $\begin{array}{llllll}\mathrm{pH} & 5.5 \text { and then activated with 400/100 } \mathrm{mM} \text { EDC/NHS }\end{array}$ [1-ethyl-3-(3-dimethylaminopropyl) carbodiimide hydrochloride/ $N$-Hydroxysuccinimide]. After ectodomain coupling, excess reactive esters were deactivated with $100 \mathrm{mM}$ ethanolamine $\mathrm{pH}$ 8. The amount of immobilized protein ranged from 700 to 3000 response unit (RU). $1 \mathrm{RU}$ corresponds to approximately $1 \mathrm{pg}$ of protein per $\mathrm{mm}^{2}$. Surface plasmon resonance (SPR) experiments were performed at a constant temperature of $25{ }^{\circ} \mathrm{C}$ on a MX96 (IBIS Technologies). G2 was flowed over the sensor chip as analyte at concentrations ranging from $20 \mathrm{pM}$ to $200 \mathrm{nM}$ for $60 \mathrm{~min}$ in buffer containing $25 \mathrm{mM}$ phosphate-buffered saline $\mathrm{pH} 8.0$ and $0.005 \%$ Tween 20. After each injection, an 8 min dissociation time was set, followed by a regeneration step of $30 \mathrm{sec}$ with $50 \mathrm{mM}$ acetic acid buffer, $\mathrm{pH}$ 4.5. Calibration of sensor signals and reference subtraction was evaluated with SprintX (IBIS Technologies), and further analyses was performed in Scrubber 2 (BioLogic Software). Association and dissociation rate constants $\left(\mathrm{k}_{\mathrm{a}} / \mathrm{k}_{\mathrm{d}}\right)$ were determined by globally fitting the SPR data to a 1:1 Langmuir binding model. The dissociation constant $\left(\mathrm{K}_{\mathrm{D}}\right)$ was calculated from the $k_{a}$ and $k_{d}$ parameters. In addition, the $K_{D}$ was determined from the steady state analyte binding levels (averaged between 40 to $60 \mathrm{~min}$ association time) plotted against concentration and by fitting a one-site saturation model.

\section{Conjugation of the VHH to PS IRDye700DX}

VHH were site-directionally conjugated to the photosensitizer IRDye700DXmaleimide (LI-COR Biosciences). First, the VHHs were incubated with an 2.75-fold molar 
excess of Tris(2-carboxyethyl)phosphine hydrochloride (TCEP, VWR International, Radnor, Pennsylvania, USA) to reduce the C-terminal cysteine upon which the VHH were incubated with a 4-fold molar excess of IRDye700DX-maleimide for $16 \mathrm{~h}$ at $4{ }^{\circ} \mathrm{C}$. Free label was removed by size exclusion chromatography using 3 consequent Zeba desalting columns (Thermo Fisher Scientific) according to the manufacturers protocols. Degree of conjugation determined on the Multiskan Go spectrophotometer (Thermo Fisher Scientific) and the amount of free dye was determined after size separation by SDS-PAGE (Bio-Rad) on the Odyssey scanner (Li-COR Biosciences). Afterwards, the SDS-PAGE gel was stained with Page Blue (Thermo Fisher Scientific) to show total protein. For the internalization assay, G2c was conjugated to HiLyte Fluor 647 (HL647)-maleimide (Eurogentec, Liege, Belgium) according to the protocol described above.

\section{Internalization assay}

MKN45 cells were seeded in 8 well chamber slide (Lab-Tek, Nunc) 2 days before the experiment. Cells were incubated with $1 \mu \mathrm{M}$ of G2-Alexa647 conjugate for $2 \mathrm{~h}$ at $37^{\circ} \mathrm{C}$. Unbound conjugate was removed by 3 times washing with PBS. Then, cells were fixed in 4\% PFA for $10 \mathrm{~min}$ at RT. PFA-induced autofluorescence was quenched using $100 \mathrm{mM}$ Glycine in PBS (10 min, RT). Cells were washed with PBS and then incubated with DAPI $(0.25 \mu \mathrm{g} / \mathrm{ml}$, Thermo Scientific) for $10 \mathrm{~min}$ at RT. The slides were mounted using SlowFade (Invitrogen) and pictures were taken with LSM700 confocal microscope using 63x oil immersion objective (Carl Zeiss Microscopy, Jena, Germany). The images were analyzed with ImageJ.

\section{Photodynamic therapy}

Photodynamic therapy was performed similarly to as previously described $[7,8]$. Cells (12000/well) were seeded in 96-wells cell culture plates (Greiner) and allowed to adhere overnight. Cells were then pulsed with VHH-PS in medium without phenol red for 2 $\mathrm{h}$ at $37{ }^{\circ} \mathrm{C}$ after which unbound VHH-PS was removed by washing the cells twice with $100 \mu \mathrm{l}$ medium. Bound VHH-PS was then detected using the Odyssey scanner and an EVOS fluorescence microscope (Thermo Fischer Scientific). Cells were illuminated for $1 \mathrm{~h}$ using a 690 diode laser (Modulight, Tampere, Finland) with a $5 \mathrm{~mW} / \mathrm{cm}^{2}$ fluence rate for a total light dose of $18 \mathrm{~J} / \mathrm{cm}^{2}$ and were incubated overnight at $37{ }^{\circ} \mathrm{C}$ in the cell culture incubator. The next day, the cells were screened for viability. For this, a) cells were incubated with calcein (Thermo Fisher Scientific) and propidium iodide (Thermo Fisher 
Scientific) to stain live and dead cells and were subsequently imaged on an EVOS fluorescence microscope. Also, b) cell viability was quantified using the AlamarBlue ${ }^{\circledR}$ reagent (Thermo Fisher Scientific), which was quantified using an FluoStar Optima plate reader (BMG Labtech, Ortenberg, Germany). Data was plotted and fitted using Prism 7 software (GraphPad, La Jolla, California, USA).

\section{Results}

Three previously selected VHHs recognizing the Met ectodomain were taken for further characterization, i.e. E9, G2 and F5 [25]. These VHHs were recloned in an E. coli production vector and binding to the human Met ectodomain was assessed by ELISA (Figure 1A-C). The two VHH clones E9 and G2 bound human Met ectodomain with apparent binding affinities $\left(\mathrm{K}_{\mathrm{D}}\right)$ of respectively $7 \pm 3 \mathrm{nM}$ and $2.2 \pm 1 \mathrm{nM}$. The $\mathrm{K}_{\mathrm{D}}$ value of F5 could not be determined properly but was estimated to be at least higher than $50 \mathrm{nM}$. To investigate to which subdomain these VHHs bind, ELISAs on chimeric human/llama Met ectodomains were performed. In these chimeric constructs, either the first 122 (LS1) or 473 (LS5) amino acids of human Met were replaced by those of llama Met (Figure 1B) [32]. Integrity of these constructs was confirmed by the binding of the anti-Met antibody c224G11 (ABT-700 or Telisotuzumab), which is directed against the first IPT region (Figure 1C, left) [32-34]. c224G11 showed a similar binding affinity to either human Met or the two chimeric mutants, whereas no binding of c224G11 to llama Met was observed. Because the VHHs were raised in llama, no cross-reactivity of these VHHs to llama Met or parts of llama Met was expected. The binding affinity and $\mathrm{B}_{\max }$ of all three VHHs was completely lost for LS5 and strongly reduced on LS1, as compared to human Met, suggesting that the binding epitopes for all three VHHs involve propeller blades 2-6 of the SEMA subdomain. Due to its high affinity for Met and the additional characterization performed previously [25], G2 was selected as the lead clone for Mettargeted PDT. 


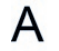

G2:

Anti-Met VHH G2-FLAG-His (AAADYKDDDDKGAAHHHHHH) $17.1 \mathrm{kDa}$

Vector: pMEK222 Host: E.Coli TG1

\begin{tabular}{|l|l|l|l|}
\hline LS & anti-Met VHH G2 & FLAG & 6xHis \\
\hline
\end{tabular}

\section{G2c:}

Anti-Met VHH G2-C-Direct (ACAxxxxEPEA)

$15.8 \mathrm{kDa}$

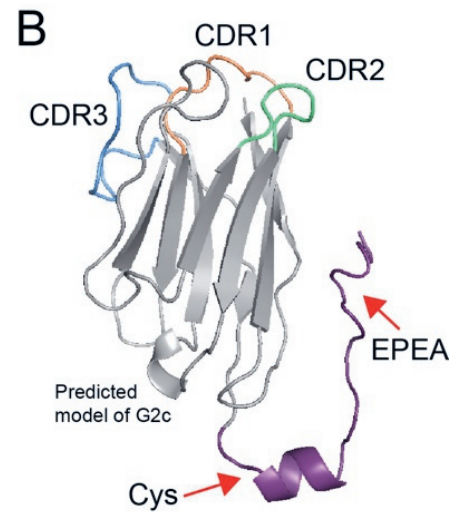

Figure 2. Production of G2 with a C-terminal tag containing a free thiol $(\mathrm{G} 2 \mathrm{c})$ and conjugation to PS. (A) Schematic representation of the anti-Met VHH G2, expressed as a FLAG-His tagged protein (top) and G2c carrying an additional cysteine in its C-terminal C-Direct tag. The molecular weight of the proteins are indicated, as well as the expression vector and production organism used. LS, pelB leader sequence. SS, Suc2 secretion sequence. (B) Predicted model of G2c in which the C-terminal tag (purple) containing the unpaired cysteine and the EPEA affinity tag is indicated with red arrows. CDRs are located on the opposing end of the VHH. The model was based on the structure of NB10 (85\% amino acid sequence homology with QME-G2) as published by Park et al. (PDB ID 4DKA:A, C-score 0.13) [27].

We examined the propensity of G2c to interact with the four variants of Met ectodomain (human, llama, LS1 and LS5) using Surface Plasmon resonance (SPR). In agreement with the ELISA data (Figure 1), the SPR experiments confirm that G2c interacts with both human Met and the LS1 variant but not with llama Met or the LS5 variant (Figure 3A), suggesting the involvement of propeller blades 2-6 of the Met SEMA subdomain to be involved in the binding of G2c. While G2c showed similar dissociation rates for human Met and LS1, the association constant was four times lower towards LS1 compared to human Met (Figure 3B and $\mathbf{C}$ ). Kinetic parameters calculated at equilibrium conditions (Figure 3D) showed that the binding affinity of G2c for LS1 is lower as compared to its affinity to human Met $\left(\mathrm{K}_{\mathrm{D}}\right.$ of $2.3 \pm 0.1 \mathrm{nM}$ for human Met and $4.7 \pm 0.5 \mathrm{nM}$ for $\mathrm{LS} 1, \mathrm{n}=3$ ). In conclusion, addition of a cysteine residue to the $\mathrm{C}$-terminal region of $\mathrm{G} 2$ did not affect binding affinity to human Met nor did it affect domain specificity. 


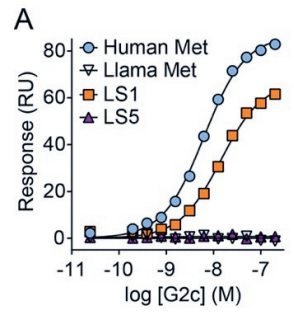

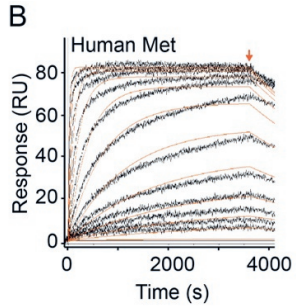

$$
\begin{array}{ll}
\mathrm{K}_{\mathrm{a}}: & 2.0 \pm 0.2 \cdot 10^{-5} \mathrm{M}^{-1} \mathrm{~s}^{-1} \\
\mathrm{~K}_{\mathrm{d}}: & 3.5 \pm 0.4 \cdot 10^{-4} \mathrm{~s}^{-1} \\
\mathrm{~K}_{\mathrm{D}}: & 1.8 \pm 0.1 \mathrm{nM}
\end{array}
$$

C

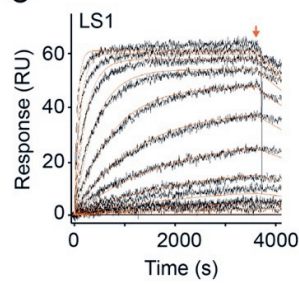

$\mathrm{K}_{\mathrm{a}}: \quad 8.4 \pm 0.1 \cdot 10^{-5} \mathrm{M}^{-1} \mathrm{~s}^{-1}$

$\mathrm{K}_{\mathrm{d}}: 2.6 \pm 0.5 \cdot 10^{-4} \mathrm{~s}^{-1}$
D

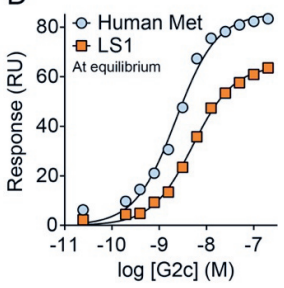

$\mathrm{K}_{\mathrm{D}} \mathrm{hMet}: 2.3 \pm 0.1 \mathrm{nM}$ $\mathrm{K}_{\mathrm{D}} \mathrm{LS} 1: 4.7 \pm 0.5 \mathrm{nM}$

Figure 3. Binding analysis of G2c on Met using Surface Plasmon Resonance (SPR). (A) Binding of G2c to wildtype human Met ectodomain and the LS1 llama/human chimera, but not the llama or the LS5 chimeric Met ectodomain proteins. (B and C) Representative SPR sensorgrams of the association phase and dissociation phase (starting at arrow) of G2c binding to human Met (B) and LS1 chimera (C). Kinetic fitting is shown in orange. (D) Equilibrium binding plot of G2c to human Met and LS1 chimeric variant. Kinetic parameters and equilibrium dissociation constants are the average \pm SD of three independent measurements.

Subsequently, the free thiol in G2c was conjugated to the water-soluble PS IRDye700DX using maleimide chemistry, resulting in a degree of conjugation of $\sim 1$, as determined spectrophotometrically. However, because $\sim 40 \%$ of the signal in the solution was still free PS (Figure 4A), a degree of conjugation of $\sim 0.6$ would be a more realistic estimation. Because of the hydrophylic nature of IRDye700DX and the lack of toxicity observed for free PS in our previous study [7], we decided to continue with the conjugated G2c-PS. Conjugation of PS to G2c only mildly affected its apparent binding affinity for Met, as determined by ELISA (apparent affinity values of $2.2 \pm 0.2 \mathrm{nM}$ for G2, $1.8 \pm 0.1$ $\mathrm{nM}$ for $\mathrm{G} 2 \mathrm{c}$ and $5.9 \pm 0.3 \mathrm{nM}$ for G2c-PS, Figure 4B).
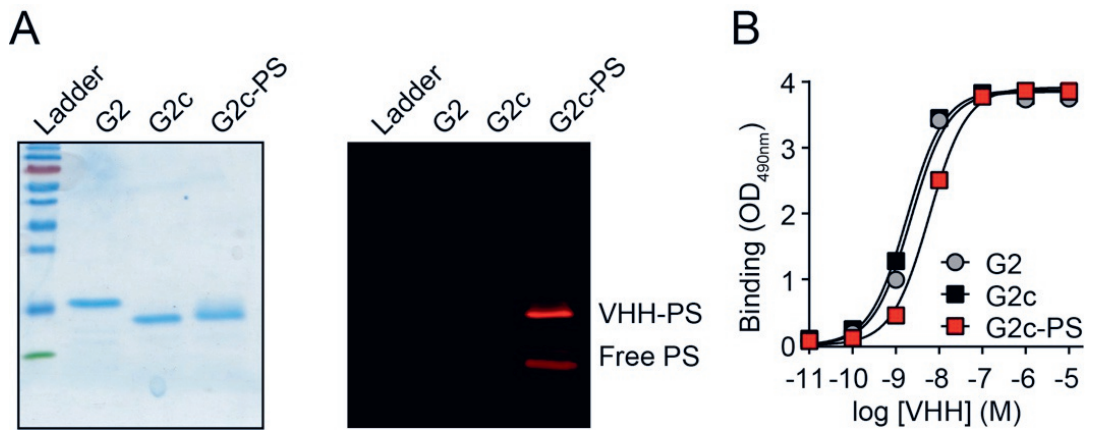

Figure 4. Conjugation of G2c to photosensitizer IRDye700DX. (A, left) Reducing SDS-PAGE gel showing purified G2 from E. coli, G2c from S. cerevisiae and G2c-PS. Size-separated proteins were stained with PageBlue stain. (A, right) G2c-PS and free PS in the gel shown on the left, as detected on an Odyssey scanner before the PageBlue stain. (B) Binding of G2, G2c and G2c-PS to Met ectodomain in ELISA (representative figure, mean \pm SD). 
In addition, G2c-PS was also still able to bind to the Met-overexpressing MKN45 cells (Figure 5A). No binding of G2c was detected on Met-negative TOV112D cells. For the in vitro PDT experiments, cells were pulsed with a concentration range of G2c-PS for $1 \mathrm{~h}$ at $37^{\circ} \mathrm{C}$, a pulse duration that reflects the circulation of VHHs in blood and the time required for tumor uptake of fluorescently labeled VHHs [35]. Besides acting as a PS via the production of reactive oxygen species, IRDye700DX is also a fluorophore and can therefore be used for detecting binding of the conjugate to cells. During the pulse, G2c-PS was able to associate with Met-expressing MKN45 cells with half-max signals being obtained at concentrations comparable to the binding affinity of the $\mathrm{VHH}$ for Met ectodomain (Figure 5B). Little association of G2c-PS was observed for the Met-negative TOV112D cells. Uptake of G2c-PS by MKN45 cells was initially suggested in wide field microscopy images (Figure 5C), which was confirmed by confocal microscopy imaging (Figure 5D).
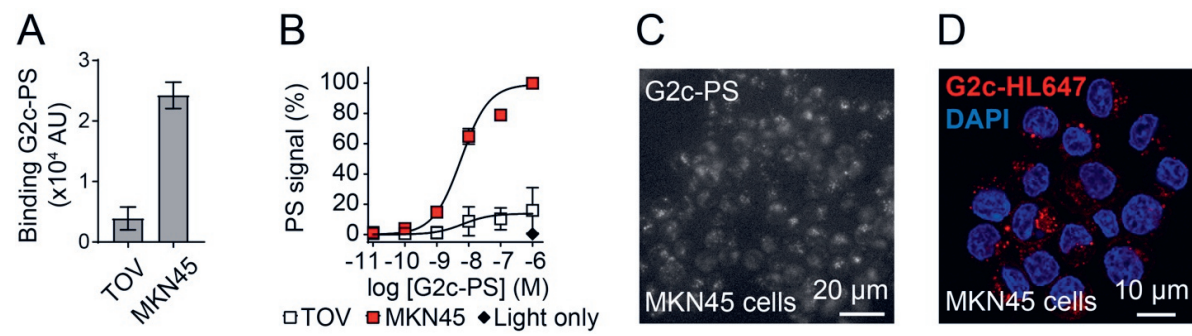

Figure 5. Met-targeting G2c-PS conjugates associate with Met-overexpressing MKN45 cells. (A) Binding of G2c-PS to MKN45 cells and not to TOV112D cells. Cells were incubated with $50 \mathrm{nM}$ G2c-PS for $2 \mathrm{~h}$ on ice and PS fluorescence was detected with an Odyssey infra-red scanner. Mean \pm $\mathrm{SD}$ of $\mathrm{n}=2$ is shown. (B) Increased cell association of G2c-PS with MKN45 cells with increasing concentrations. MKN45 or TOV112D cells were pulsed with G2c-PS for $2 \mathrm{~h}$ at $37{ }^{\circ} \mathrm{C}$. PS fluorescence was detected with an Odyssey infra-red scanner. Mean \pm SD of $n=3$ is shown. (C) Association of G2c-PS in MKN45 cells. Cells were pulsed for $1 \mathrm{~h}$ with $1 \mu \mathrm{M}$ of G2c-PS and imaged by widefield fluorescent microscopy. (D) Uptake of G2c-HL647 in MKN45 cells. Cells were pulsed for $2 \mathrm{~h}$ with $1 \mu \mathrm{M}$ of G2c-HL647 (red) and imaged by confocal laser scanning microscopy.

Exposure of MKN45 cells to different concentrations of G2c-PS, combined with illumination, resulted in $\sim 0 \%$ cell viability at nanomolar concentrations (Figure 6A). This was also confirmed by fluorescence microscopy, in which almost all cells pulsed with G2cPS and illuminated showed uptake of propidium iodide and absence of calcein staining (Figure 6B). We subsequently assessed the ability of G2c-PS to induce cell death of two other Met-expressing cell lines. Although showing lower EC $_{50}$ values compared MKN45 
cells, G2c-PS could reduce the viability of the previously described TOV+Met cell line and the liver hepatocellular carcinoma cell line HepG2 in a dose-dependent manner (Figure 6C).
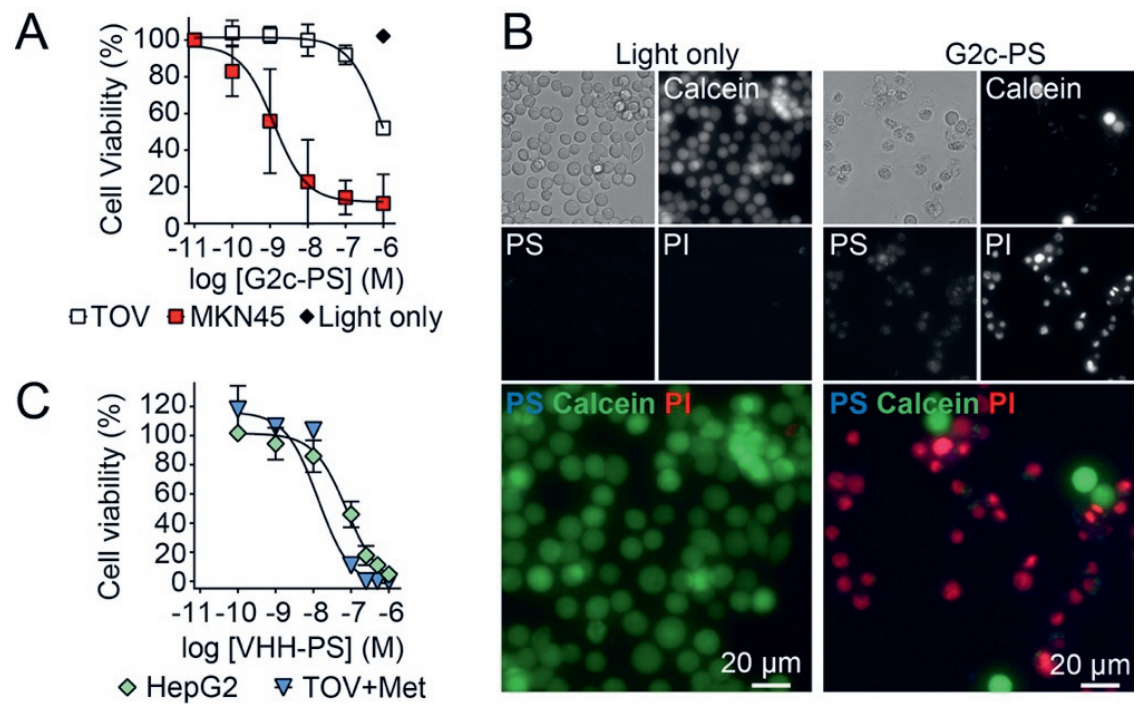

Figure 6. Met-targeting G2c-PS induce cell death of Met-expressing cells upon activation by illumination (A) Selective killing of Met-overexpressing MKN45 tumor cells and not of Met-negative TOV112D cells by G2c-targeted PDT. Cells were pulsed for $2 \mathrm{~h}$ at $37{ }^{\circ} \mathrm{C}$ and subsequently illuminated to activate the PS. Cell viability was assessed 1 day later. Mean \pm SD of $n=3$ is shown. (B) Phase contrast and fluorescence microscopy (EVOS) of PDT-treated MKN45 cells showing G2cPS (blue in merge, visible as magenta (red+blue), live cells (calcein, green) and dead cells (propidium iodide, red). (C) Induction of cell death of Met expressing TOV+Met and HepG2 cells by G2c-PS upon a $2 \mathrm{~h}$ pulse followed by PS activation by illumination. Representative plot of $n=2$.

\section{Discussion}

PDT is valuable method for inducing local cell death of malignant cells by the local activation of PS. Unfortunately, clinically employed PS are administered systemically, are relatively hydrophobic, and passively accumulate in the tumor as a result of their hydrophobicity in combination with the enhanced permeability and retention (EPR) effect [36,37]. This results in sub-optimal tumor uptake of PS, off-target toxicity and long photosensitivity [38]. To enhance the specific tumor uptake of photosensitizers, PSdelivering nanocarriers have been developed and water soluble PS have been conjugated to tumor-specific peptides or antibodies (the latter is also known as photo-immunotherapy, or 
PIT) [39-41]. Because the extent of damage caused by PDT is correlated to the amount of PS delivered, ideal targets for these approaches are highly expressed on tumor cells and preferably in a homogeneous fashion throughout the solid tumor mass. Examples of tumor targets are the typical tumor-related receptors EGFR [42,43] and HER2 [44], but also interleukin-2 receptor [43] and carcinoembryonic antigen have been used as valuable tumor biomarkers [45-47]. In some types of cancers (e.g. non-small cell lung cancer, or colorectal cancer), treatment with anti-EGFR therapy resulted in the intratumoral, clonal selection of therapy resistant cells [48]. This can be the result of activating mutations of signaling proteins from the EGFR signaling pathways, such as KRAS. Alternatively, other receptor tyrosine kinases (RTK) can become activated and, in most of these cases, upregulation of the Met receptor tyrosine kinase is regarded as one of the main mechanisms for this type of resistance [49-52]. The Met RTK is also considered as a good tumor target and therapeutic anti-Met antibodies have been developed [32,34,53]. In order to expand the possible therapeutic strategies for many of those cancers, we here evaluated whether Met could also serve as a target for targeted PDT.

Targeted PDT using antibodies as targeting agents, i.e. PIT, is currently being evaluated in the clinic for head and neck cancer (NCT02422979). These results are eagerly awaited, as these are the first tests in patients and thus can significantly advance this field of targeted PDT. Nevertheless, we consider there is necessity for further improvements, and in the current study, we have employed nanobodies or VHHs as targeting agents. Conventional antibodies of the IgG type are large, dimeric molecules, typically designed for extended blood circulation. These characteristics may lead to slow tumor penetration/accumulation, poor tumor to normal tissue ratios, and long photosensitivity [54]. Reduction of the duration of photosensitivity might be achieved by the rapid tumor accumulation and subsequent rapid clearance of the PS from the body. Antibody fragments smaller than IgG like Fabs, scFv or $\mathrm{VHH} /$ nanobodies have these properties without compromising their affinity for their tumor biomarkers [55]. As we have described before, particularly the VHHs are indeed characterized by rapid tumor accumulation, rapid blood clearance, combined with the ability to bind targets with high affinity $[16,35]$. Another argument for using VHHs over conventional antibody fragments, might be the short distance between the conjugated PS and the paratope on the target $(\sim 4 \mathrm{~nm})$. Because the reactive oxygen species generated by PS are short lived ( $<40 \mathrm{~ns})$ and may travel only a 
short distance $(<20 \mathrm{~nm})$, the delivery of the PS close to the cell membrane or to vital cell organelles might favor its potency [56,57]. For that same reason, it would be of interest to assess the potency of Met-targeting PS conjugates recognizing epitopes closer to the membrane than the SEMA subdomain, such as the PSI or IPT subdomains [23]. This would require new PSI or IPT specific VHHs.

By using chimeric constructs, consisting of different human and Llama Met fragments, we were able to show that all three Met targeting VHHs used in this study bind the SEMA subdomain of the Met-ectodomain. The best SEMA binder, i.e. G2, was provided with a C-terminal tag containing an unpaired cystein to allow conjugation to the PS using maleimide chemistry. The addition of this thiol-containing C-Direct tag did not affect the binding characteristics of the $\mathrm{VHH}$, which is in line with previous studies of Cterminally labeled VHH via an unpaired cysteine [14-16]. Multiple methods have been described to site directional functionalize antibody fragments $[15,58]$. In our experience, incorporation of an unpaired cysteine in the C-terminal tag, as we described in this study, allowed the straightforward production, purification and conjugation to commercially available tracers with the use of a single tag $[15,16,59]$.

The binding affinity of G2c for Met was determined on purified ectodomains using two different technologies: ELISA and SPR. In these experiments, the apparent binding affinity of G2c in ELISA was comparable (low nanomolar range) to the $\mathrm{K}_{\mathrm{D}}$ values determined under equilibrium by SPR. The SPR analysis does however allow the determination of the association and dissociation rate constants, which provided additional information on the binding kinetics of G2c on wild type and chimeric Met proteins. Moreover, similar affinities for G2 were found for binding to Met-expressing cells [25], indicating that cellular components do not influence VHH binding.

Application of the anti-Met conjugate G2c-PS in in vitro PDT resulted in the efficient and specific killing of the Met overexpressing MKN45 cells and the Metexpressing TOV+Met and HepG2 cells, while the Met negative cell line TOV112D remained undisturbed. The observation that the treated cells could be stained with propidium iodide suggest cells could have died through necrosis. Further studies would be needed to determine the exact mechanism of cell death. The potency of G2c-PS in killing MKN45 cells was in the nanomolar range. In the two other cell lines, the Met expressing HepG2 tumor cells and the TOV112D cells stably expressing Met (TOV+Met), the 
observed potencies were lower. These differences in potencies might be related to the relative Met expression levels of these cells. MKN45 is an highly Met-overexpressing cell line due to genomic amplification of the Met gene [60]. It would be interesting to assess the potencies and efficacies of Met-targeted PDT on a wider range of Met-expressing tumor cells types. As a consequence of the high expression in MKN45 cells, the receptor is constitutively auto-active and internalized, allowing uptake and intracellular accumulation of the Met-targeted PS [61]. This is in line with what we have observed for G2c-PS and G2c-HL647. As the subcellular localization of PS can influence the mechanism of cell death and the overall potency of the PDT, as suggested in our previous study with different formats of EGFR-targeted VHHs [62], it would be interesting to determine the contribution of PS-uptake in the observed effects. The potency and efficacy of the VHH-PS conjugates could be further enhanced by employing a mixture of these Met-targeting conjugates with the previously developed EGFR targeting ones. This could also affect a larger population of tumor cells, including cells that upregulated Met expression as resistance mechanism against anti-EGFR therapy and could be an advantage in tumors with heterogeneously expressed markers, such as EGFR and Met [24,51].

In conclusion, this study has demonstrated that targeting Met using sitedirectionally conjugated VHH-PS has the ability to specifically kill Met overexpressing tumor cells. Follow up studies should evaluate the potency of this approach in more relevant models (in vivo). Taken together, Met-targeted PDT might serve as alternative or complementary approach for combating cancer.

\section{Acknowledgments}

This project has received funding from the European Research Council (ERC) under the European Union's Horizon 2020 research and innovation program (grant agreement No 677582).

\section{References}

1. van Straten, D.; Mashayekhi, V.; de Bruijn, H.S.; Oliveira, S.; Robinson, D.J. Oncologic photodynamic therapy: Basic principles, current clinical status and future directions. Cancers (Basel). 2017, 9(2), 19.

2. Agostinis, P.; Berg, K.; Cengel, K.A.; Foster, T.H.; Girotti, A.W.; Gollnick, S.O.; Hahn, S.M.; Hamblin, M.R.; Juzeniene, A.; Kessel, D.; et al. Photodynamic therapy of cancer: an update. $C A$ Cancer J Clin 2011, 61, 250-281. 
3. Kwiatkowski, S.; Knap, B.; Przystupski, D.; Saczko, J.; Kedzierska, E.; Knap-Czop, K.; Kotlinska, J.; Michel, O.; Kotowski, K.; Kulbacka, J. Photodynamic therapy - mechanisms, photosensitizers and combinations. Biomed Pharmacother 2018, 106, 1098-1107.

4. Gomer, C.J. Preclinical examination of first and second generation photosensitizers used in photodynamic therapy. Photochem Photobiol 1991, 54, 1093-1107.

5. Githaka, N.; Konnai, S.; Skilton, R.; Kariuki, E.; Kanduma, E.; Murata, S.; Ohashi, K. Genotypic variations in field isolates of Theileria species infecting giraffes (Giraffa camelopardalis tippelskirchi and Giraffa camelopardalis reticulata) in Kenya. Parasitol. Int. 2013, 62, 448-453.

6. Bechet, D.; Couleaud, P.; Frochot, C.; Viriot, M.L.; Guillemin, F.; Barberi-Heyob, M. Nanoparticles as vehicles for delivery of photodynamic therapy agents. Trends Biotechnol. 2008, 26, 612-621.

7. Heukers, R.; van Bergen en Henegouwen, P.M.P.; Oliveira, S. Nanobody-targeted photodynamic therapy for oncology. Photodiagnosis Photodyn. Ther. 2015, 12, 339.

8. Van Driel, P.B.A.A.; Boonstra, M.C.; Slooter, M.D.; Heukers, R.; Stammes, M.A.; Snoeks, T.J.A.; De Bruijn, H.S.; Van Diest, P.J.; Vahrmeijer, A.L.; Van Bergen En Henegouwen, P.M.P.; et al. EGFR targeted nanobody-photosensitizer conjugates for photodynamic therapy in a pre-clinical model of head and neck cancer. J. Control. Release 2016, 229, 93-105.

9. Hamers-Casterman, C.; Atarhouch, T.; Muyldermans, S.; Robinson, G.; Hammers, C.; Songa, E.B.; Bendahman, N.; Hammers, R. Naturally occurring antibodies devoid of light chains. Nature 1993, 363, 446-448.

10. Muyldermans, S. Nanobodies: Natural Single-Domain Antibodies. Annu. Rev. Biochem. 2013, $82,775-797$.

11. Harmsen, M.M.; De Haard, H.J. Properties, production, and applications of camelid singledomain antibody fragments. Appl. Microbiol. Biotechnol. 2007, 77, 13-22.

12. De Groeve, K.; Deschacht, N.; De Koninck, C.; Caveliers, V.; Lahoutte, T.; Devoogdt, N.; Muyldermans, S.; De Baetselier, P.; Raes, G. Nanobodies as tools for in vivo imaging of specific immune cell types. J Nucl Med 2010, 51, 782-789.

13. Massa, S.; Vikani, N.; Betti, C.; Ballet, S.; Vanderhaegen, S.; Steyaert, J.; Descamps, B.; Vanhove, C.; Bunschoten, A.; van Leeuwen, F.W.B.; et al. Sortase A-mediated site-specific labeling of camelid single-domain antibody-fragments: a versatile strategy for multiple molecular imaging modalities. Contrast Media Mol. Imaging 2016, 11, 328-339.

14. Massa, S.; Xavier, C.; De Vos, J.; Caveliers, V.; Lahoutte, T.; Muyldermans, S.; Devoogdt, N. Site-specific labeling of cysteine-tagged camelid single-domain antibody-fragments for use in molecular imaging. Bioconjug. Chem. 2014, 25, 979-988.

15. Van Lith, S.A.M.; Van Duijnhoven, S.M.J.; Navis, A.C.; Leenders, W.P.J.; Dolk, E.; Wennink, J.W.H.; Van Nostrum, C.F.; Van Hest, J.C.M. Legomedicine - A Versatile Chemo-Enzymatic Approach for the Preparation of Targeted Dual-Labeled Llama Antibody-Nanoparticle Conjugates. Bioconjug. Chem. 2017, 28, 539-548.

16. Kijanka, M.; Warnders, F.J.; El Khattabi, M.; Lub-De Hooge, M.; Van Dam, G.M.; Ntziachristos, V.; De Vries, L.; Oliveira, S.; Van Bergen En Henegouwen, P.M.P. Rapid optical imaging of human breast tumour xenografts using anti-HER2 VHHs site-directly conjugated to IRDye $800 \mathrm{CW}$ for image-guided surgery. Eur. J. Nucl. Med. Mol. Imaging 2013, 40, 1718 1729 .

17. Köstler, W.J.; Yarden, Y. The epidermal growth factor receptor family. In Handbook of Cell Signaling, 2/e; 2010; Vol. 2, pp. 435-441 ISBN 9780123741455.

18. Bublil, E.M.; Yarden, Y. The EGF receptor family: spearheading a merger of signaling and therapeutics. Curr. Opin. Cell Biol. 2007, 19, 124-134.

19. Peruzzi, B.; Bottaro, D.P. Targeting the c-Met signaling pathway in cancer. Clin. Cancer Res. 2006, 12, 3657-3660.

20. Birchmeier, C.; Birchmeier, W.; Gherardi, E.; Vande Woude, G.F. Met, metastasis, motility and more. Nat. Rev. Mol. Cell Biol. 2003, 4, 915-925.

21. Cooper, C.S.; Park, M.; Blair, D.G.; Tainsky, M.A.; Huebner, K.; Croce, C.M.; Vande Woude, G.F. Molecular cloning of a new transforming gene from a chemically transformed human cell 
line. Nature 1984, 311, 29-33.

22. Stamos, J.; Lazarus, R.A.; Yao, X.; Kirchhofer, D.; Wiesmann, C. Crystal structure of the HGF $\beta$-chain in complex with the Sema domain of the Met receptor. EMBO J. 2004, 23, 2325-2335.

23. Gherardi, E.; Youles, M.E.; Miguel, R.N.; Blundell, T.L.; Iamele, L.; Gough, J.; Bandyopadhyay, A.; Hartmann, G.; Butler, P.J.G. Functional map and domain structure of MET, the product of the c-met protooncogene and receptor for hepatocyte growth factor/scatter factor. Proc. Natl. Acad. Sci. 2003, 100, 12039-12044.

24. Bradley, C.A.; Salto-Tellez, M.; Laurent-Puig, P.; Bardelli, A.; Rolfo, C.; Tabernero, J.; Khawaja, H.A.; Lawler, M.; Johnston, P.G.; Van Schaeybroeck, S. Targeting c-MET in gastrointestinal tumours: Rationale, opportunities and challenges. Nat. Rev. Clin. Oncol. 2017, $14,562-576$.

25. Heukers, R.; Altintas, I.; Raghoenath, S.; De Zan, E.; Pepermans, R.; Roovers, R.C.; Haselberg, R.; Hennink, W.E.; Schiffelers, R.M.; Kok, R.J.; et al. Targeting hepatocyte growth factor receptor (Met) positive tumor cells using internalizing nanobody-decorated albumin nanoparticles. Biomaterials 2014, 35, 601-10.

26. Roy, A.; Kucukural, A.; Zhang, Y. I-TASSER: A unified platform for automated protein structure and function prediction. Nat. Protoc. 2010, 5, 725-738.

27. Park, Y.J.; Budiarto, T.; Wu, M.; Pardon, E.; Steyaert, J.; Hol, W.G.J. The structure of the Cterminal domain of the largest editosome interaction protein and its role in promoting RNA binding by RNA-editing ligase L2. Nucleic Acids Res. 2012, 40, 6966-6977.

28. Gorlani, A.; Lutje Hulsik, D.; Adams, H.; Vriend, G.; Hermans, P.; Verrips, T. Antibody engineering reveals the important role of $\mathrm{J}$ segments in the production efficiency of llama singledomain antibodies in Saccharomyces cerevisiae. Protein Eng. Des. Sel. 2012, 25, 39-46.

29. Thomassen, Y.E.; Meijer, W.; Sierkstra, L.; Verrips, C.T. Large-scale production of VHH antibody fragments by Saccharomyces cerevisiae. In Enzyme and Microbial Technology; 2002; Vol. 30, pp. 273-278.

30. Van De Laar, T.; Visser, C.; Holster, M.; López, C.G.; Kreuning, D.; Sierkstra, L.; Lindner, N.; Verrips, T. Increased heterologous protein production by Saccharomyces cerevisiae growing on ethanol as sole carbon source. Biotechnol. Bioeng. 2007, 96, 483-494.

31. Gorlani, A.; De Haard, H.; Verrips, T. Expression of VHHs in saccharomyces cerevisiae. Methods Mol. Biol. 2012, 911, 277-286.

32. Basilico, C.; Hultberg, A.; Blanchetot, C.; De Jonge, N.; Festjens, E.; Hanssens, V.; Osepa, S.I.; De Boeck, G.; Mira, A.; Cazzanti, M.; et al. Four individually druggable MET hotspots mediate HGF-driven tumor progression. J. Clin. Invest. 2014, 124, 3172-3186.

33. Gonzalez, A.; Broussas, M.; Beau-Larvor, C.; Haeuw, J.F.; Boute, N.; Robert, A.; Champion, T.; Beck, A.; Bailly, C.; Corvaïa, N.; et al. A novel antagonist anti-cMet antibody with antitumor activities targeting both ligand-dependent and ligand-independent c-Met receptors. Int. J. Cancer 2016, 139, 1851-1863.

34. Prat, M.; Oltolina, F.; Basilico, C. Monoclonal Antibodies against the MET/HGF Receptor and Its Ligand: Multitask Tools with Applications from Basic Research to Therapy. Biomedicines 2014, 2, 359-383.

35. Oliveira, S.; van Dongen, G.A.M.S.; Stigter-van Walsum, M.; Roovers, R.C.; Stam, J.C.; Mali, W.; van Diest, P.J.; van Bergen en Henegouwen, P.M.P. Rapid visualization of human tumor xenografts through optical imaging with a near-infrared fluorescent anti-epidermal growth factor receptor nanobody. Mol. Imaging 2012, 11, 33-46.

36. Fang, J.; Nakamura, H.; Maeda, H. The EPR effect: Unique features of tumor blood vessels for drug delivery, factors involved, and limitations and augmentation of the effect. Adv. Drug Deliv. Rev. 2011, 63, 136-151.

37. Iyer, A.K.; Khaled, G.; Fang, J.; Maeda, H. Exploiting the enhanced permeability and retention effect for tumor targeting. Drug Discov. Today 2006, 11, 812-818.

38. Allison, R.R.; Sibata, C.H. Oncologic photodynamic therapy photosensitizers: A clinical review. Photodiagnosis Photodyn. Ther. 2010, 7, 61-75.

39. Shirasu, N.; Nam, S.O.; Kuroki, M. Tumor-targeted photodynamic therapy. In Anticancer Research; 2013; Vol. 33, pp. 2823-2832. 
40. Bugaj, A.M. Targeted photodynamic therapy - A promising strategy of tumor treatment. Photochem. Photobiol. Sci. 2011, 10, 1097-1109.

41. Lucky, S.S.; Soo, K.C.; Zhang, Y. Nanoparticles in photodynamic therapy. Chem. Rev. 2015, 115, 1990-2042.

42. Savellano, M.D.; Hasan, T. Photochemical targeting of epidermal growth factor receptor: A mechanistic study. Clin. Cancer Res. 2005, 11, 1658-1668.

43. Nakajima, T.; Sano, K.; Choyke, P.L.; Kobayashi, H. Improving the efficacy of photoimmunotherapy (PIT) using a cocktail of antibody conjugates in a multiple antigen tumor model. Theranostics 2013, 3, 357-365.

44. Savellano, M.D.; Pogue, B.W.; Hoopes, P.J.; Vitetta, E.S.; Paulsen, K.D. Multiepitope HER2 targeting enhances photoimmunotherapy of HER2-overexpressing cancer cells with pyropheophorbide-a immunoconjugates. Cancer Res. 2005, 65, 6371-6379.

45. Shirasu, N.; Yamada, H.; Shibaguchi, H.; Kuroki, M.; Kuroki, M. Potent and specific antitumor effect of CEA-targeted photoimmunotherapy. Int. J. Cancer 2014, 135, 2697-2710.

46. Maawy, A.A.; Hiroshima, Y.; Zhang, Y.; Heim, R.; Makings, L.; Garcia-Guzman, M.; Luiken, G.A.; Kobayashi, H.; Hoffman, R.M.; Bouvet, M. Near infra-red photoimmunotherapy with anti-CEA-IR700 results in extensive tumor lysis and a significant decrease in tumor burden in orthotopic mouse models of pancreatic cancer. PLoS One 2015, 10, 1-10.

47. Carcenac, M.; Larroque, C.; Langlois, R.; Van Lier, J.E.; Artus, J.C.; Pèlegrin, A. Preparation, phototoxicity and biodistribution studies of anti-carcinoembryonic antigen monoclonal antibodyphthalocyanine conjugates. Photochem. Photobiol. 1999, 70, 930-936.

48. Brand, T.M.; Iida, M.; Wheeler, D.L. Molecular mechanisms of resistance to the EGFR monoclonal antibody cetuximab. Cancer Biol. Ther. 2011, 11, 777-792.

49. Misale, S.; Di Nicolantonio, F.; Sartore-Bianchi, A.; Siena, S.; Bardelli, A. Resistance to AntiEGFR therapy in colorectal cancer: From heterogeneity to convergent evolution. Cancer Discov. 2014, 4, 1269-1280.

50. Bardelli, A.; Corso, S.; Bertotti, A.; Hobor, S.; Valtorta, E.; Siravegna, G.; Sartore-Bianchi, A.; Scala, E.; Cassingena, A.; Zecchin, D.; et al. Amplification of the MET receptor drives resistance to anti-EGFR therapies in colorectal cancer. Cancer Discov. 2013, 3, 658-673.

51. Wang, S.; Song, Y.; Yan, F.; Liu, D. Mechanisms of resistance to third-generation EGFR tyrosine kinase inhibitors. Front. Med. 2016, 10, 383-388.

52. Matsubara, D.; Ishikawa, S.; Sachiko, O.; Aburatani, H.; Fukayama, M.; Niki, T. Co-activation of epidermal growth factor receptor and c-MET defines a distinct subset of lung adenocarcinomas. Am. J. Pathol. 2010, 177, 2191-2204.

53. Hultberg, A.; Morello, V.; Huyghe, L.; De Jonge, N.; Blanchetot, C.; Hanssens, V.; De Boeck, G.; Silence, K.; Festjens, E.; Heukers, R.; et al. Depleting MET-expressing tumor cells by ADCC provides a therapeutic advantage over inhibiting HGF/MET signaling. Cancer Res. 2015, 75, 3373-3383.

54. Van Dongen, G.A.M.S.; Visser, G.W.M.; Vrouenraets, M.B. Photosensitizer-antibody conjugates for detection and therapy of cancer. Adv. Drug Deliv. Rev. 2004, 56, 31-52.

55. Wu, A.M.; Senter, P.D. Arming antibodies: Prospects and challenges for immunoconjugates. Nat. Biotechnol. 2005, 23, 1137-1146.

56. Redmond, R.W.; Kochevar, I.E. Spatially Resolved Cellular Responses to Singlet Oxygen. Photochem. Photobiol. 2006, 82, 1178.

57. Louryan, S. La formation de la tête des vertébrés: Faits et hypothèses. Rev. Med. Brux. 2005, 26 , 98-102.

58. Tsuchikama, K.; An, Z. Antibody-drug conjugates: recent advances in conjugation and linker chemistries. Protein Cell 2018, 9, 33-46.

59. van Brussel, A.S.A.; Adams, A.; Oliveira, S.; Dorresteijn, B.; El Khattabi, M.; Vermeulen, J.F.; van der Wall, E.; Mali, W.P.T.M.; Derksen, P.W.B.; van Diest, P.J.; et al. Hypoxia-Targeting Fluorescent Nanobodies for Optical Molecular Imaging of Pre-Invasive Breast Cancer. Mol. Imaging Biol. 2016, 18, 535-544.

60. Rege-Cambrin, G.; Scaravaglio, P.; Carozzi, F.; Giordano, S.; Ponzetto, C.; Comoglio, P.M.; Saglio, G. Karyotypic analysis of gastric carcinoma cell lines carrying an amplified c-met 
oncogene. Cancer Genet. Cytogenet. 1992, 64, 170-173.

61. Smolen, G.A.; Sordella, R.; Muir, B.; Mohapatra, G.; Barmettler, A.; Archibald, H.; Kim, W.J.; Okimoto, R.A.; Bell, D.W.; Sgroi, D.C.; et al. Amplification of MET may identify a subset of cancers with extreme sensitivity to the selective tyrosine kinase inhibitor PHA-665752. Proc. Natl. Acad. Sci. U.S. A. 2006, 103, 2316-21.

62. Heukers, R.; van Bergen en Henegouwen, P.M.P.; Oliveira, S. Nanobody-photosensitizer conjugates for targeted photodynamic therapy. Nanomedicine Nanotechnology, Biol. Med. 2014, $10,1441-1451$. 


\section{Chapter 4}

Acute cellular and vascular responses to photodynamic therapy using EGFR-targeted nanobody-photosensitizer conjugates studied with intravital optical imaging and magnetic resonance imaging

Henriette S. de Bruijn, Vida Mashayekhi, Tom J.L. Schreurs, Pieter B.A.A. van Driel, Gustav J. Strijkers, Paul J. van Diest, Clemens W.G.M. Lowik, Ann L.B. Seynhaeve, Timo L.M. ten Hagen, Jeanine J. Prompers, Paul M.P. van Bergen en Henegouwen, Dominic J. Robinson, Sabrina Oliveira

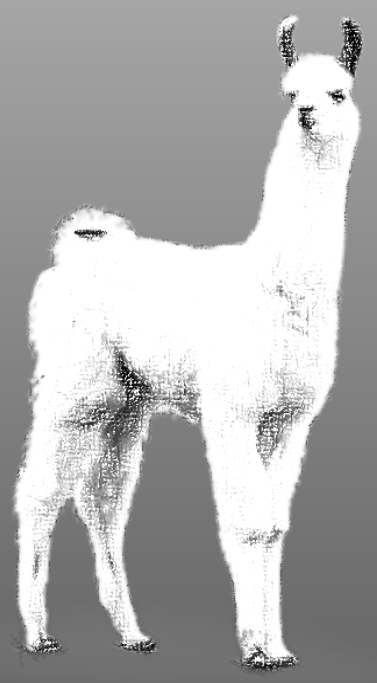




\section{ABSTRACT}

Targeted photodynamic therapy (PDT) has the potential to selectively damage tumor tissue and to increase tumor vessel permeability. Here we characterize the tissue biodistribution of two EGFR-targeted nanobody-photosensitizer conjugates (NB-PS), the monovalent 7D12-PS and the biparatopic 7D12-9G8-PS. In addition, we report on the local and acute phototoxic effects triggered by illumination of these NB-PS which have previously shown to lead to extensive tumor damage. Intravital microscopy and the skin-fold chamber model, containing OSC-19-luc2-cGFP tumors, were used to investigate a) the fluorescence kinetics and distribution, b) the vascular response and c) the induction of necrosis after illumination at 1 or $24 \mathrm{~h}$ post administration of 7D12-PS and 7D12-9G8-PS. In addition, dynamic contrast enhanced magnetic resonance imaging (DCE-MRI) of a solid tumor model was used to investigate the microvascular status $2 \mathrm{~h}$ after 7D12-PS mediated PDT. Image analysis showed significant tumor colocalization for both NB-PS which was higher for 7D12-9G8-PS. Intravital imaging showed clear tumor cell membrane localization 1 and $2 \mathrm{~h}$ after administration of 7D12-9G8-PS, and fluorescence in or close to endothelial cells in normal tissue for both NB-PS. PDT lead to vasoconstriction and leakage of tumor and normal tissue vessels in the skin-fold chamber model. DCE-MRI confirmed the reduction of tumor perfusion after 7D12-PS mediated PDT. PDT induced extensive tumor necrosis and moderate normal tissue damage, which was similar for both NB-PS conjugates. This was significantly reduced when illumination was performed at $24 \mathrm{~h}$ compared to $1 \mathrm{~h}$ after administration. Although differences were observed in distribution of the two NB-PS conjugates, both led to similar necrosis. Clearly, the response to PDT using NB-PS conjugates is the result of a complex mixture of tumor cell responses and vascular effects, which is likely to be necessary for a maximally effective treatment. 


\section{Introduction}

Targeted strategies to deliver a photosensitizer to tumor cells have the potential to improve the therapeutic effect of photodynamic therapy (PDT) [1-5]. PDT involves the administration of a photosensitizer and the application of light with the appropriate wavelength $[1,2]$. After absorption of light by the photosensitizer, a range of photochemical reactions occur that, in the presence of oxygen, leads to the formation of predominantly singlet oxygen $[1,2]$. The highly reactive singlet oxygen causes damage to nearby proteins and lipids resulting in cellular, vascular and immunological responses that ultimately encompass the PDT response [1]. In general, clinically used photosensitizers are hydrophobic, which promotes cell binding but provides no tumor specificity. As a result, a 2 to 4 days interval between photosensitizer administration and light delivery is common to obtain an optimum tumor to normal tissue ratio. In addition, patients become light sensitive, for several weeks, due to skin-photosensitization. Increased tumor specific uptake of the photosensitizer has the potential to lead to significantly better tumor responses, much less normal tissue damage and a decreased skin photosensitization [1-3]. Increased expression levels of specific receptors on tumor cells can be used to target these cells. In head and neck cancer, $83 \%$ of tumors show overexpression of the epidermal growth factor receptor (EGFR), which is commonly used as a target for various targeted therapies [6]. For targeted PDT a range of approaches have been explored, such as the direct conjugation of photosensitizer to antibodies or peptides, or targeted drug delivery systems where multiple photosensitizer are loaded into a liposome or other nanoparticles [4,5,7-14].

We have been developing an alternative approach using nanobodies [15-18]. Nanobodies are the smallest naturally derived antigen-binding fragments that consist of the variable domain of a heavy-chain antibody [19]. The characteristics of nanobodies make them favorable for targeted drug delivery as they bind specifically and with high affinity to their antigens, are relatively small, stable, water soluble and have low immunogenic potential [19-22]. The monovalent 7D12 and the biparatopic 7D12-9G8 nanobodies specifically target EGFR and compete with EGF for binding to EGFR [23]. In contrast to the monovalent 7D12 nanobody, the biparatopic 7D12-9G8 leads to a more rapid EGFR internalization by inducing receptor clustering [15,24]. After conjugation to the water soluble photosensitizer IRDye700DX, a silicon-phthalocyanine derivative that absorbs and 
emits NIR light, these nanobodies act as a carrier of photosensitizer and can be used for targeted PDT or tumor targeted fluorescence guided imaging $[4,25,26]$.

Recently we have shown promising results in vitro and in vivo using nanobodyphotosensitizer (NB-PS), as an alternative approach for targeted PDT [15,16]. In vitro we have shown a clear relationship between level of EGFR expression, fluorescence intensity and PDT efficacy for both 7D12-PS and 7D12-9G8-PS [15]. Subsequently, in an in vivo study employing an orthotopic tongue model transplanted with an oral squamous cell carcinoma expressing green fluorescent protein (OSC-19-luc2-cGFP), we used quantitative fluorescence spectroscopy to determine the NB-PS distribution in time after administration [16]. The fluorescence intensity in tumor and normal skin tissue was significantly higher for 7D12-9G8-PS compared to 7D12-PS. 7D12-PS showed a peak fluorescence intensity in the tongue tumor already at $30 \mathrm{~min}$ after administration after which it slowly decreased. 7D12-9G8-PS showed a high fluorescence intensity in the tumor up to $4 \mathrm{~h}$ after administration after which it started to decrease. The tumor to normal ratio at $1 \mathrm{~h}$ after administration was $1.8 \pm 0.3$ and $3.8 \pm 0.5$, respectively. Although the tumor to normal ratio increased to $16.1 \pm 4.5$ and $30.8 \pm 0.9$, respectively at $24 \mathrm{~h}$ after administration, the tumor fluorescence intensity was significantly lower. Therefore, in that study, light was applied $1 \mathrm{~h}$ after administration for both NB-PS. Histological examination $24 \mathrm{~h}$ after PDT showed extensive tumor necrosis and damage to the vasculature in and close to the tumor [16].

These promising results encouraged us to further investigate, in the present study, the distribution of the NB-PS conjugates and PDT-induced response in vivo within tumor and normal tissue. The (sub-) cellular localization of photosensitizer is considered to be important as it determines the initial site of photodamage due to the short diffusion distance of singlet oxygen [27]. Antitumor effects induced by PDT are known to be mediated not only by cellular damage but also by vascular responses [1]. Interestingly, PDT, and more recently targeted PDT, have been shown to be capable of increasing the enhanced permeability and retention (EPR) effect by improving tumor vessel permeability [28-31]. Since this could potentiate the delivery of other nanomedicines to the tumor site in future combined therapies, in this study we also carefully investigate the vascular effects of NB targeted PDT. 
Intravital imaging in a skin-fold chamber model can be used to provide longitudinal information on the kinetics and localization of fluorophores in detail in a living animal, and be used to investigate direct effects on the vasculature [8,32-39]. Therefore, in the present study, we used intravital imaging in the mouse skin-fold chamber model transplanted with the OSC-19-luc2-cGFP tumor, a tumor model we have previously investigated in the mouse tongue [16]. We investigated the biodistribution of the conjugates and the vascular responses induced by NB-PS mediated PDT; constriction and leakage, as well as the induction of necrosis after illumination at 1 or $24 \mathrm{~h}$ post administration [16,4043]. To complement intravital microscopy, contrast-enhanced MRI and dynamic contrast enhanced (DCE) MRI were also used, as these have shown to be effective tools to determine the vascular effects and measure the microvascular status of tumors early after PDT [43-50]. We employed the same tumor cell line but now grown subcutaneously in mice for DCE-MRI, to interrogate the microvascular status of the tumor and underlying muscle [50]. Here, the degree of blood perfusion and vessel integrity can be quantified by measuring the tissue influx and wash out of a small gadolinium-based contrast agent. Histological examination of tissues collected directly after or up to $48 \mathrm{~h}$ after PDT was also used to determine the tumor necrotic fraction, vascular perfusion and damage to endothelial and normal tissue in both models.

\section{Experimental section}

\section{Study design}

Intravital microscopy and DCE-MRI were used to investigate the distribution and the direct effects of two EGFR-targeted NB-PS conjugates.

Intravital microscopy combined with the skin-fold chamber tumor model was used to study fluorescence distribution and PDT response longitudinally. Mice wearing the skinfold chamber were divided over 6 groups (Table 1). Fluorescence distribution was investigated intravitally in groups 1-3 by imaging at 1,2 and $24 \mathrm{~h}$ after administration of the NB-PS and before PDT. After the last time-point (24 h) PDT was performed in an attempt to use the high tumor to normal ratio (TNR) observed previously [16]. In groups 4-6 PDT was performed $1 \mathrm{~h}$ after administration. Groups 3 and 6 were light only controls. PDT responses were investigated intravitally in all groups by imaging directly, 2, 24 and $48 \mathrm{~h}$ after PDT. At $2 \mathrm{~h}$ post illumination tetramethylrhodamine dextran was administered 
and imaged in all groups to investigate vascular leakage and flow. At $24 \mathrm{~h}$ post illumination the necrosis marker HQ4 $[41,42]$ was administered and imaged $24 \mathrm{~h}$ later. Tissue was harvested for ex vivo necrosis marker imaging and histological analysis.

Table 1. Overview of nanobody-photosensitizer treatment schemes studied intravitally for distribution and tumor and normal tissue damage.

\begin{tabular}{cccccc} 
Group & $\mathrm{n}$ & $\begin{array}{c}\text { Nanobody-PS } \\
\text { conjugate }\end{array}$ & $\begin{array}{c}\text { Illumination } \\
\text { at }\end{array}$ & HQ4 & $\begin{array}{c}\text { Harvest } \\
\text { after ill. }\end{array}$ \\
\hline 1 & $8^{*}, * *$ & 7D12-PS & $24 \mathrm{~h}$ & Yes & $48 \mathrm{~h}$ \\
\hline 2 & $8^{*}, * *$ & 7D12-9G8-PS & $24 \mathrm{~h}$ & Yes & $48 \mathrm{~h}$ \\
\hline 3 & 3 & Saline & $24 \mathrm{~h}$ & Yes & $48 \mathrm{~h}$ \\
\hline 4 & $8 *, * *$ & 7D12-PS & $1 \mathrm{~h}$ & Yes & $48 \mathrm{~h}$ \\
\hline 5 & $8 *, * *$ & 7D12-9G8-PS & $1 \mathrm{~h}$ & Yes & $48 \mathrm{~h}$ \\
\hline 6 & 3 & Saline & $1 \mathrm{~h}$ & No & $48 \mathrm{~h}$
\end{tabular}

* 1 animal without tumor, serving as normal tissue control

** 1 animal sacrificed before PDT for fluorescence distribution

DCE-MR imaging of the solid tumor model was used to investigate the PDT response in 2 groups. One group of animals $(n=5)$ was injected with 7D12-PS and the control group $(n=4)$ received saline. For practical reasons only one of the two NB conjugates could be investigated, and 7D12-PS was chosen. To compensate for the prolonged gas-anesthesia necessary for the procedure, illumination was performed at $2 \mathrm{~h}$ instead of $1 \mathrm{~h}$ after administration. MR imaging was performed on 3 consecutive days, 1 day before illumination, approximately $1 \mathrm{~h}$ after illumination inside the bore of the scanner on the second day and 1 day after illumination, after which animals were sacrificed. Histological analysis was performed on a selection of two control animals and three 7D12-PS mediated PDT-treated animals, which received a perfusion marker (Hoechst 33342) 5 min prior to sacrifice.

\section{Nanobody conjugates}

The nanobody-photosensitizer conjugates (NB-PS) 7D12-PS and 7D12-9G8-PS were prepared as described previously $[15,16]$. His-tagged nanobodies were produced in E. coli BL21and purified from the periplasmic fraction using Nickel-NTA agarose [23,51]. The monovalent nanobody 7D12 binds to domain III of the EGFR, preventing EGF- 
binding to the receptor [52]. The biparatopic nanobody 7D12-9G8 was composed of two nanobodies that bind to different epitopes on domain III and due to the short linker sequence they cannot bind simultaneously to the same receptor, therefore being able to create clusters of receptors [52]. Conjugation of photosensitizer to the nanobodies was performed as described in Heukers et al. 2014 [15], except that the molar ratios for conjugation was 1 to 4 for 7D12 and 1 to 3 for 7D12-9G8. In short, the nanobodies were incubated with the photosensitizer IRDye700DX (LI-COR Biosciences, Lincoln, Nebraska) for $2 \mathrm{~h}$ at room temperature. Afterwards, the NB-PS conjugates were separated from the free photosensitizer using three consequent Zeba spin desalting columns (ThermoFisher Scientific, Perbio Science Nederland). The degree of the conjugation was determined using a Nanodrop spectrophotometer as recommended by the provider by measuring the absorbance at $280 \mathrm{~nm}$ and $689 \mathrm{~nm}$. The purity of the NB-PS conjugates was determined on 15\% SDS-PAGE and imaged on an Odyssey infrared scanner (LI-COR Biosciences) using $700 \mathrm{~nm}$. Afterwards, PageBlue staining was performed to show the total protein content. The concentration of NB-PS administered was corrected for the degree of conjugation, so that every mouse received the same amount of photosensitizer, i.e. $6 \mathrm{nmol}$ IRDye700DX).

\section{Cell line}

The oral squamous cell carcinoma line, OSC-19-luc2-cGFP, was cultured in Dulbecco's Modified Eagle's Medium (containing 4.5 g D-Glucose/L, 110 mg Sodium Pyruvate/L) plus GlutaMAX ${ }^{\mathrm{TM}}$, supplemented with 10\% Fetal Bovine Serum, 1x Minimal Essential Medium non-essential amino acids solution and 1x Minimal Essential Medium vitamin solution as before [53].

\section{Intravital microscopy}

\section{Skin-fold chamber model}

The mouse skin-fold chamber was prepared on the back of female Balb/c nu or $\mathrm{BalB} / \mathrm{cAnNRj}$ mice and transplanted with the oral squamous cell carcinoma (OSC-19-luc2cGFP) using a procedure adapted from previous studies [54-56]. In brief, mice received analgesia $1 \mathrm{~h}(1 \mathrm{mg} / \mathrm{kg}$ rimadyl cattle s.c; Pfizer, Capelle a/d IJssel, NL) and anesthesia (75 mg/kg ketamine i.p.; Alfasan, Woerden, NL and $1 \mathrm{mg} / \mathrm{kg}$ medetomidine i.p.; Eurovet, Bladel, NL) 20 min before the procedure. The dorsal skin was folded and fixed between two frames after removal of one side of the skin in $1 \mathrm{~cm}$ diameter up to the fascia of the opposed skin. A tumor cell suspension of $5 \times 10^{4}$ cells in $10 \mu 1$ was injected superficially 
in the fascia/subcutaneous musculature and the window was closed with a sterile microscope cover glass of $12 \mathrm{~mm}$ diameter secured with a retaining ring. Glass spacers (thick cover glasses of $9 \mathrm{~mm}$ diameter) were placed on the epidermal side of the skin and another $12 \mathrm{~mm}$ circular microscopic cover glass was used to close the window on that side. Mice were housed individually in climate-controlled cabinets with an ambient temperature of $30^{\circ} \mathrm{C}$ and a humidity of $70 \%$.

Experiments started 6-9 days after preparation of the chambers. Mice were awake during all $i . v$. administrations. From two weeks prior to the experiments, all mice were fed a chlorophyll free diet (catalogue number 4208.00, Hope Farms b.v., Woerden, NL or \#100208, Altromin, Germany) to minimize the contribution of pheophorbides to the autofluorescence emission spectrum. The animal ethics committee of the Erasmus MC approved the experimental protocols of the study.

\section{Fluorescence distribution}

The microscopic distribution of 7D12-PS and 7D12-9G8-PS was intravitally imaged under gas anesthesia at 1,2 and $24 \mathrm{~h}$ after i.v. administration. Mice injected with physiological saline served as a control. A Zeiss Laser Scanner Confocal Microscope 510 equipped with 2x,10x and 20x air Plan-Neofluar objectives, a heated stage and a gas anesthesia supply unit was used. Fluorescence images were recorded with the $2.5 \mathrm{x}$ (whole chamber), 10x ( 1 or 2 tumor areas of maximal $7.6 \mathrm{~mm}^{2}$ and 1 normal tissue area) and 20x objectives ( 3 z-stacks of 7 slices, 2 in tumor and 1 in normal tissue) using $633 \mathrm{~nm}$ excitation and long pass $650 \mathrm{~nm}$ detection for the nanobody-conjugates and $488 \mathrm{~nm}$ excitation and 505-530 $\mathrm{nm}$ band pass detection for the GFP signal of the tumor. Corresponding transmission images were recorded for orientation purposes using $488 \mathrm{~nm}$ light. Fluorescence and transmission images were recorded before, 1, 2 and $24 \mathrm{~h}$ after i.v. administration of the nanobody-conjugates under $2-3 \%$ isoflurane in oxygen anesthesia. Reference standards were recorded every day to correct for differences between experiments. Images were analyzed using ImageJ-Fiji. Regions of interest (ROI) were drawn around tumor and normal tissue areas excluding the large venules and arteries based on the transmission and GFP images. The ROI's for normal tissue were drawn in regions without any GFP signal and distant from larger blood vessels. Integrated density was determined and normalized to an area of $5000 \mu \mathrm{m}^{2}$ rather than the mean fluorescence intensity of the pixels since the fluorescence was expected to be heterogeneous due to the 
receptor specific binding of the nanobody conjugates and the spatial resolution of the images. The integrated density was corrected for the dark current, variation in the reference standard and the individual autofluorescence. For each animal, time point and tissue type, 2-6 regions of interest's were drawn and averaged. Colocalization analysis between the GFP and red fluorescence signals was performed after correction for the dark current, using the Coloc 2 plugin.

In parallel, for ex vivo microscopy, skin-fold chamber tissue of 4 mice was dissected at 1 or $24 \mathrm{~h}$ after administration of 7D12-PS or 7D12-9G8-PS (group 1, 2, 4 and 5). Tissue was snap-frozen and stored at $-80{ }^{\circ} \mathrm{C}$. Cryosections were made and stained with anti-CD31 Brilliant Violet (AntibodyChain BV, The Netherlands) for fluorescence microscopy. Images were collected using a Leica SP5 Microscope equipped with 40x oil Plan-Neofluar objective, $633 \mathrm{~nm}$ excitation and 650-800 $\mathrm{nm}$ HyD detection for the NBconjugates and $405 \mathrm{~nm}$ excitation and 420-450 nm detection for the Brilliant Violet signal. Reference standards were recorded to correct for differences between experiments.

\section{$\underline{\text { PDT }}$}

Illumination was performed under gas anesthesia on the microscope stage 1 or $24 \mathrm{~h}$ after i.v. administration of 7D12-PS or 7D12-9G8-PS. A $690 \mathrm{~nm}$ laser (ML7700, Modulight, Inc. Tampere, Finland) and a frontal light distributor (Medlight SA, Ecublens, Switzerland) was used to deliver a dose of $100{\mathrm{~J} . \mathrm{cm}^{-2}}^{2}$ at an irradiance of $50 \mathrm{~mW} . \mathrm{cm}^{-2}$. Mice injected with physiological saline served as a light only control.

\section{Cellular response to PDT}

The GFP signal of the tumor cells was used to observe changes in the morphology in time after PDT. Necrosis was detected intravitally using the necrosis avid agent HQ4 $(20 \mathrm{nMol} /$ mouse $[41,42])$ i.v. administered $24 \mathrm{~h}$ post PDT and imaged $24 \mathrm{~h}$ later using $633 \mathrm{~nm}$ excitation and $650 \mathrm{~nm}$ long pass detection. Tissue was dissected for ex vivo necrosis avid agent imaging and histological analysis. The skin-fold chamber tissue was cut in two halves through the tumor, snap-frozen in liquid nitrogen and stored in $-80{ }^{\circ} \mathrm{C}$ until sectioning. Sections were collected at three depths with alternating thickness of 50 and $8 \mu \mathrm{m}$ for HQ4 fluorescence imaging and histology respectively and stored in $-80{ }^{\circ} \mathrm{C}$ until imaging. The $50 \mu \mathrm{m}$ sections were defrosted, dried and imaged unfixed and uncovered using a Zeiss laser scanner confocal microscope. Fluorescence images were recorded with the 10x objective using $633 \mathrm{~nm}$ excitation and long pass $650 \mathrm{~nm}$ detection for HQ4 
fluorescence and $488 \mathrm{~nm}$ excitation and $505-530 \mathrm{~nm}$ band pass detection for the GFP signal of the tumor. Corresponding transmission images were recorded for orientation purposes using $488 \mathrm{~nm}$. Images were analyzed using ImageJ-Fiji. Regions of interest were drawn around tumor and normal tissue areas. The ROI's selection of tumor were created by thresholding the GFP signal and creating a mask of it to ensure that only tumor tissue was measured. The fluorescence intensity was corrected for the dark current and variation in the reference standard for the different recording days.

Thereafter tissues were formalin fixed and paraffin embedded (FFPE) for histological examination. The FFPE tissues were sectioned and stained with hematoxylin and eosin (H\&E) and analyzed as described by van Driel et al. [16].

\section{Vascular response to PDT}

The vascular responses to PDT were investigated using both transmission imaging and tetramethylrhodamine dextran imaging. Tetramethylrhodamine dextran (2000 kDa, $1 \mathrm{mg} / \mathrm{ml}, 0.1 \mathrm{mg}$ mouse, D7139, ThermoFisher Scientific, Molecular Probes, Eugene, Oregon, USA) was administered $2 \mathrm{~h}$ post PDT and imaged intravitally within 20 min using $543 \mathrm{~nm}$ excitation and 560-615 $\mathrm{nm}$ bandpass detection and the 20x objective. The number of animals showing leakage in tumor or normal tissue, i.e., tetramethylrhodamine fluorescence outside the vessels, was counted. Tumor vascular flow was scored based on the appearance of the tetramethylrhodamine fluorescence according to three criteria, i.e., lack of flow (no fluorescence in the vessels), reduced flow (disrupted fluorescence in the vessels) or normal flow (fluorescence confined to the vessels). The proportional area of tumor tissue that was affected by each criterion was estimated. Transmission images using $488 \mathrm{~nm}$ were recorded immediately, 2, 24 and $48 \mathrm{~h}$ after PDT using the $2.5 \mathrm{x}$ objective to investigate changes in vascular architecture. The severity of the changes in vascular architecture in normal tissue was scored as follows: No change in vascular lumen and flow visible (-), minimal changes in vascular lumen of the larger vessels and/or capillary shutdown (+), severe changes in vascular lumen and flow visible in areas (++) and severe changes in vascular lumen and no flow visible $(+++)$.

\section{DCE-MRI}

\section{Solid tumor model}

Female BALB/c nude mice (Charles River) aged 8-11 weeks were subcutaneously injected with $1 \times 10^{6}$ cells in the right hind limb. Tumors became palpable after 3 to 6 days 
and were measured with a caliper every 2 or 3 days. MRI experiments were started when the tumors reached a volume of approximately $300 \mathrm{~mm}^{3}$. The Animal Care and Use Committee of Maastricht University approved the protocol. All applicable institutional and/or national guidelines for the care and use of animals were followed.

PDT

Mice were anesthetized during injection of 7D12-PS or physiological saline until PDT and MRI imaging using 2-3\% isoflurane in oxygen anesthesia. Body temperature was monitored with a rectal probe and maintained at $37{ }^{\circ} \mathrm{C}$ using a warm water circuit. Respiration rate was monitored using a pressure balloon. PDT was performed $2 \mathrm{~h}$ after administration and not $1 \mathrm{~h}$ to compensate for the lower metabolism caused by the prolonged gas anesthesia needed for the procedures. Light was delivered using a $690 \mathrm{~nm}$ laser (ML7700, Modulight, Inc. Tampere, Finland) via a fiber and collimating lens into the MR magnet to a dose of $100 \mathrm{~J} . \mathrm{cm}^{-2}$ at an irradiance of $50 \mathrm{~mW} . \mathrm{cm}^{-2}$. A black paper mask was placed surrounding the tumor to protect the rest of the animal from stray light.

$\underline{\mathrm{MRI}}$

Experiments were performed using a 7T MRI scanner (BioSpec 30/70 USR, Bruker, Billerica, Massachusetts, USA) with a $72 \mathrm{~mm}$ diameter quadrature transmit and receive birdcage coil. Briefly, $T_{2}$-weighted anatomical reference scans were acquired followed by a 3D pre-contrast $T_{1}$ map. Then, a 15 min 3D DCE-MRI scan was performed with a temporal resolution of $3.5 \mathrm{~s}$, to capture the dynamic influx of contrast agent (Dotarem) in the tissue. Based on the DCE-MRI data, all pixels were classified as enhanced or non-enhanced, and the following variables were calculated for each pixel: the area under the curve (AUC) of the Dotarem concentration, and $K^{\text {trans }}$ (transfer constant describing contrast exchange between blood plasma and the extravascular extracellular space). For anatomical reference a $T_{2}$ weighted multi-slice spin echo scan was acquired with 12 to 14 axial slices, covering the entire tumor. Scan parameters were: $\mathrm{TR}=1000 \mathrm{~ms}, \mathrm{TE}=30 \mathrm{~ms}$, matrix $=128 \times 128$, slice thickness $=1.0 \mathrm{~mm}$, slice gap $=0.1 \mathrm{~mm}, \mathrm{FOV}=4 \times 4 \mathrm{~cm}^{2}$. For $T_{1^{-}}$ mapping, a 3D FLASH sequence with variable flip angle was used [57]. Sequence parameters were: TR-20 ms, TE $=3.2 \mathrm{~ms}, 7$ flip angles $\left(2^{\circ}, 3^{\circ}, 5^{\circ}, 7^{\circ}, 10^{\circ}, 13^{\circ}\right.$ and $\left.20^{\circ}\right)$, matrix $=128 \times 128 \times 39, \mathrm{FOV}=40 \times 40 \times 22 \mathrm{~mm}^{3}$. DCE-MRI was performed using the same 3D FLASH sequence with shorter TE and TR and a fixed flip angle $\left(1 \mathrm{~ms}, 3 \mathrm{~ms}\right.$ and $7^{\circ}$ respectively) and an acquisition matrix of $128 \times 128 \times 17$ (zero-filled to $128 \times 128 \times 39$ ). Two 
min after the start of the scan, a dose of $0.3 \mathrm{mmol} / \mathrm{kg}$ b.w. Dotarem (Guerbet, Villepinte, France) was injected in $5 \mathrm{~s}$ using a syringe pump (Fusion 100, Chemyx Inc., Stafford, 153 TX, USA), followed by a saline flush.

Manual segmentation of tumors was performed in ITK-SNAP [58], based on $T_{2^{-}}$ weighted spin echo images. Tumor was hyper intense compared to muscle tissue. All further analysis was performed using home-made scripts in Matlab 2016a (Mathworks, Natick, MA). Muscle ROI's of 5x5 pixels were selected in four central tumor slices, with 1 pixel spacing to the tumor border and centered on the optical axis of the light beam. $T_{I}$ maps were reconstructed pixel by pixel by fitting the data signal intensities at different FA to the spoil gradient echo sequence equation:

$$
s=\propto \sin (F A)\left(1-\exp \left(-\frac{T R}{T 1}\right)\right) /\left(1-\cos (F A) \exp \left(-\frac{T R}{T 1}\right)\right) .
$$

Dynamic changes in $R_{l}(t)=1 / T_{l}(t)$ were calculated from the DCE-MRI scan, based on the pre-contrast relaxation rate $R_{1, p r e}=1 / T_{1, p r e}$ and the signal during contrast agent influx, using the same equation. Next, Dotarem concentration curves $C(t)$ were calculated using:

$R_{l}(t)=R_{l, p r e}+r_{l} C(t)$, where $r_{l}=3.53 \mathrm{~s}^{-1} \mathrm{mM}^{-1}$ is the longitudinal relaxivity of Dotarem [59]. Pixels were classified as non-enhanced when the median of the concentration curve after time of contrast injection was smaller than twice the SD before injection. Tracer kinetic modelling was performed using the standard Tofts-Kermode model [60], to estimate $K^{\text {trans }}$ and $v_{e}$ (the volume fraction of the extravascular extracellular space). The arterial input function was defined as a bi-exponential function with amplitudes $A_{1}=5.36 \mathrm{mM}$ and $A_{2}=1.27 \mathrm{mM}$, and time constants $\tau_{1}=5.36 \mathrm{~s}$ and $\tau_{2}=915 \mathrm{~s}$, as previously described [49].

\section{Histological analysis}

The subcutaneous solid tumor tissue samples were collected $5 \mathrm{~min}$ after administration of Hoechst 33342 (i.v., 32 mg/kg b.w. B2261, Sigma Aldrich, St. Louis, MO, USA), snap-frozen in liquid nitrogen and stored in $-80{ }^{\circ} \mathrm{C}$ until sectioning and analysis. A series of sections were collected from a central and peripheral transverse plane and subjected to either hematoxylin and eosin staining (H\&E) or CD31 immunofluorescence staining to detect endothelial cells. For CD31 detection, sections were fixed in acetone ( 5 min RT), air dried, incubated with biotin-conjugated rat anti-mouse 
CD31 antibodies (1 h 1:250 RT, \#102503, Biolegend, San Diego, CA, USA) and DyLight 649-conjugated streptavidin (1 h 1:100 RT, \#405224, Biolegend). Bright field and fluorescence imaging were performed by mosaic acquisition at 40x and 20x respectively. Viable and necrotic regions in H\&E were manually segmented in ImageJ 1.51. All other data analysis, including segmentation of tumors in CD31 images were performed using home-made scripts in Matlab 2016a. In the CD31 images, tissue was classified as perfused or non-perfused by applying a manually selected threshold on the fluorescence signal intensity of Hoechst 33342.

\section{Statistics}

Results of the fluorescence intensity of NB-PS or necrosis marker are presented as weighted mean $\pm \mathrm{SD}$, weighted by the SD of the mean fluorescence intensity of 2-6 ROI's at a certain time point for a certain tissue type. For each tissue type and time point the individual fluorescence intensity at a certain time point was weighted by its SD to result in a weighted mean calculated by averaging the different ROIs and weighted by the SD of that mean. The significance of differences was determined using the student t-test/ANOVA/SNK and $\mathrm{p}<0.05$ was considered significant. The Spearman's rank correlation coefficient of the colocalization of the photosensitizer with the GFP fluorescence is presented as mean \pm SEM and statistically analyzed using the student t-test and $\mathrm{p}<0.05$ was considered significant.

\section{Results}

\section{Fluorescence distribution of NB-PS conjugates}

Intravital microscopy imaging was performed to determine potential differences in the distribution between the two EGFR-targeted NB-PS in tumor and in normal tissue immediately surrounding the tumor (Figure 1A). With respect to the intensity, both conjugates showed more fluorescence in tumor than in distant normal tissue, at either 1 or $2 \mathrm{~h}$ after administration with $\mathrm{p}=0.04$ and 0.03 for 7D12-PS, and $\mathrm{p}=0.04$ and 0.06 for 7D129G8-PS, respectively (Figure 1B). The tumor to normal tissue ratio for the two conjugates was significantly higher for 7D12-9G8-PS $(\mathrm{p}<0.01)$ but only at $2 \mathrm{~h}$ after administration. The tumor to normal tissue ratio at 1 and $2 \mathrm{~h}$ after administration was $3.0 \pm 0.7$ and $2.6 \pm$ 0.4 for 7D12-PS, and $2.9 \pm 0.6$ and $4.0 \pm 0.2$ for 7D12-9G8-PS, respectively. Comparing 
the two conjugates, more fluorescence was observed in tumor and normal tissue after 7D12-9G8-PS administration at all time points.

A

A PS/GFP
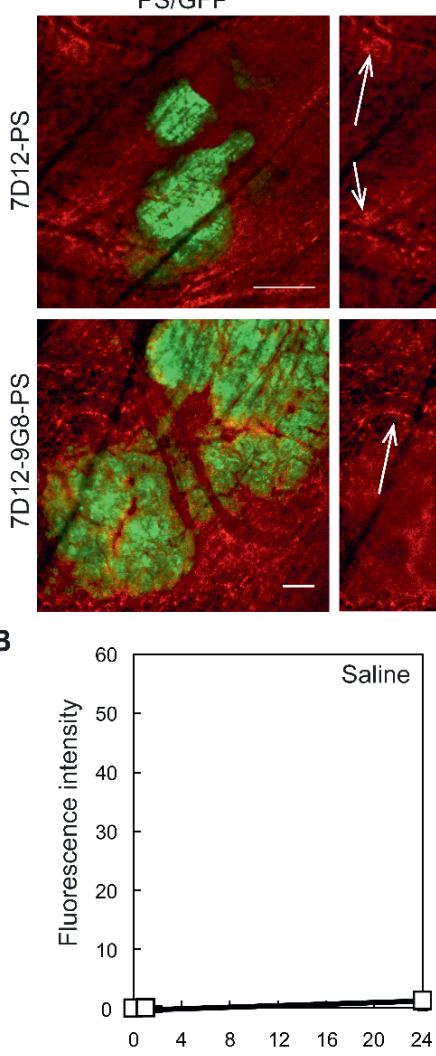

PS
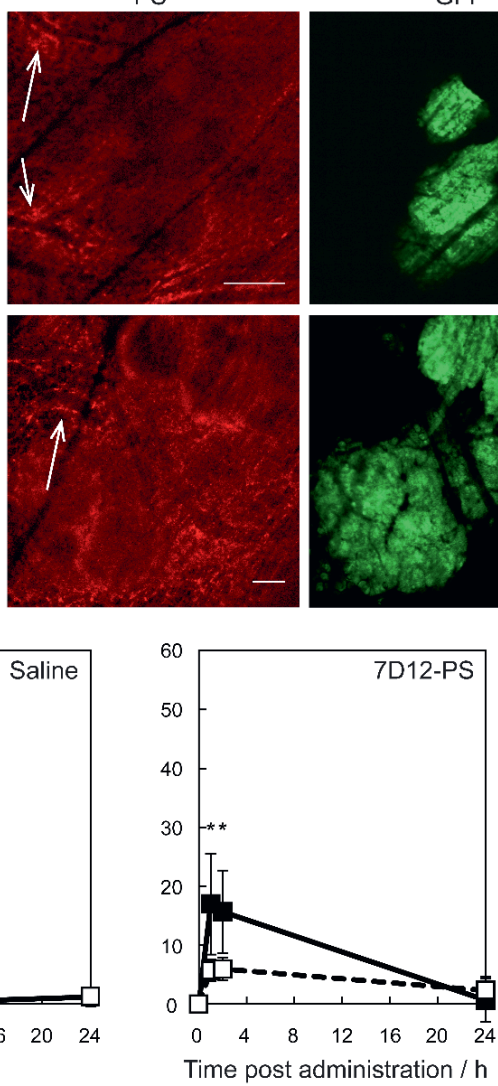

GFP
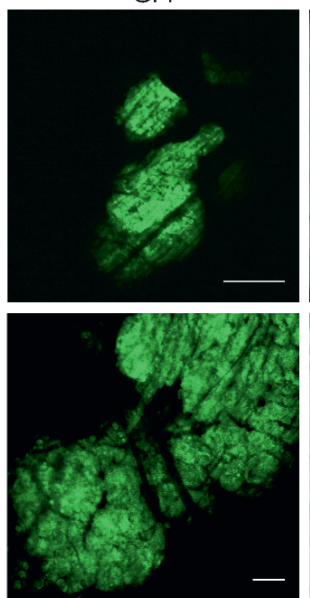

60

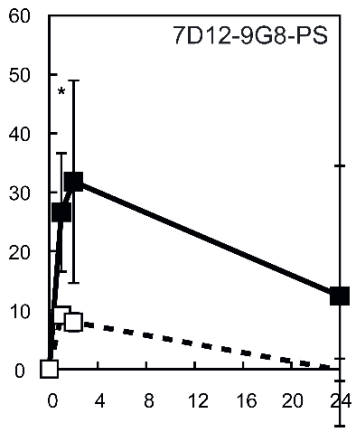
Transmission
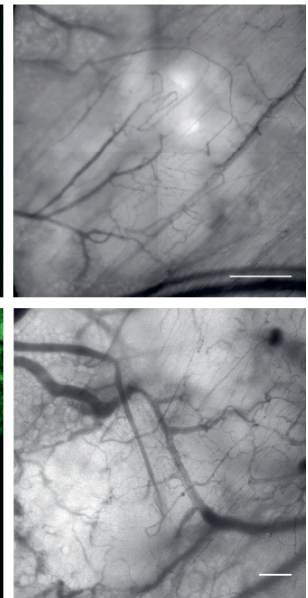

Figure 1. (A) Example of intravital fluorescence images recorded of the tumor in the skin-fold chamber $1 \mathrm{~h}$ after administration of 7D12-PS or 7D12-9G8-PS. Bar is $200 \mu \mathrm{m}$. White arrows highlight fluorescence close to vessels that surround tumor tissue. (B) Fluorescence intensity in tumor (solid squares and lines) and normal tissue far from tumor and not showing GFP signal (open squares and dashed lines) in the skin-fold chamber after administration of physiological saline, 7D12-PS or 7D12-9G8-PS. Weighted mean $\pm \mathrm{SD}, \mathrm{n}=3,6,8$ respectively. Significant differences between tumor and normal tissue with $\mathrm{p}<0.05(*)$. 
Distinct differences could be observed in the sub-cellular distribution of the two NB-PS conjugates using z-stack imaging with a higher magnification (Figure 2A). 7D129G8-PS showed an intense, membrane localized, fluorescence pattern in tumor cells. This was different after 7D12-PS administration, for which the fluorescence in the tumor tissue was more diffuse. In one animal we were able to image the internalization of 7D12-9G8-PS (Figure 2B). At $1 \mathrm{~h}$ after administration the fluorescence was visible at the membrane, and at $2 \mathrm{~h}$ fluorescence was clearly visible in the interior of the cell.

Colocalization analysis between the NB-PS fluorescence and the GFP signal in the tumor showed significant higher Spearman's rank correlation coefficients for 7D12-PS at 1 and $2 \mathrm{~h}$ after administration, compared to before administration, suggesting tumor specific binding with $\mathrm{p}<0.01$ for both time points (Figure 2C). For 7D12-9G8-PS, all time points after administration showed significant higher Spearman's rank coefficients compared to before administration with $\mathrm{p}<2 \times 10^{-6}$ for 1 and $2 \mathrm{~h}$, and $\mathrm{p}<2 \times 10^{-4}$ for $24 \mathrm{~h}$ after administration. Overall, 7D12-9G8-PS showed a significantly higher correlation between NB-PS fluorescence and GFP signal compared to 7D12-PS at all time points with $\mathrm{p}<0.003$. 
A
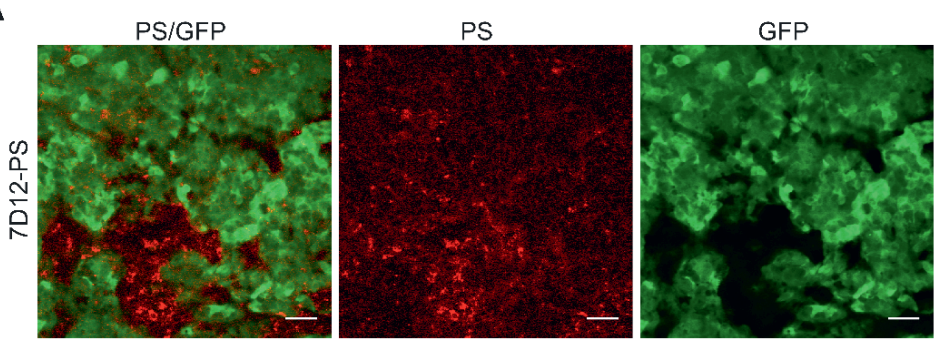

Transmission
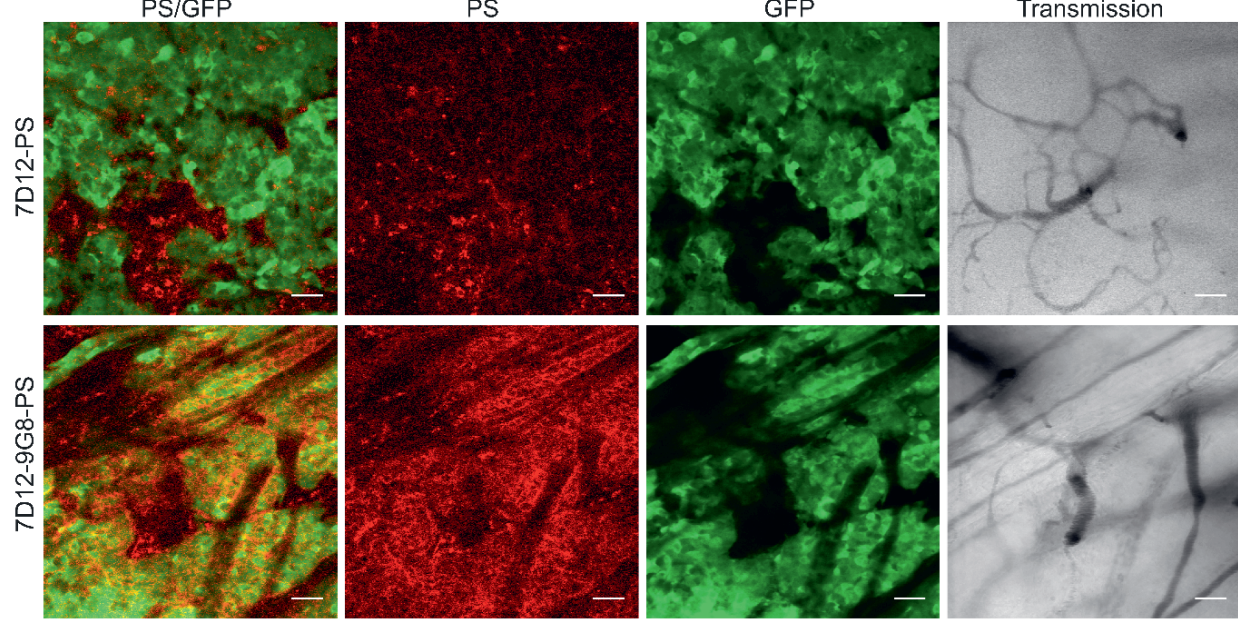

$\mathbf{B}$

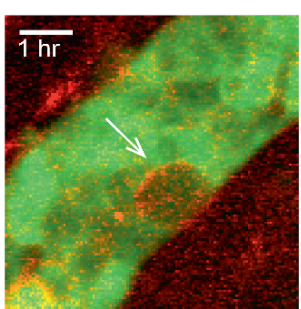

C
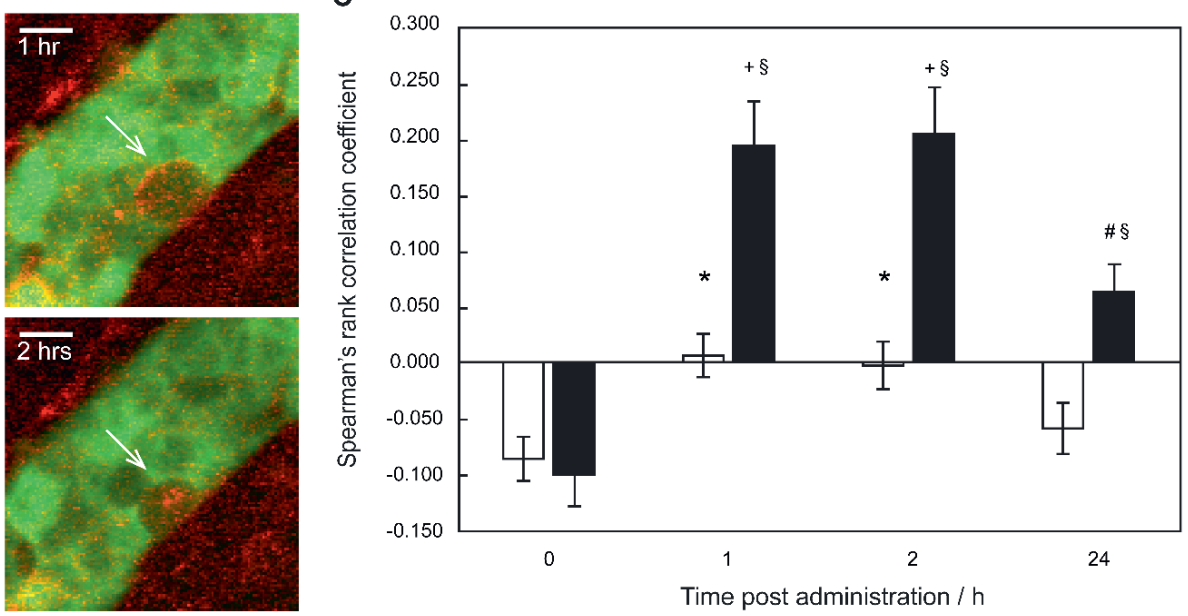

Figure 2. (A) Example of high magnification fluorescence images recorded $1 \mathrm{~h}$ after administration of 7D12-PS and 7D12-9G8-PS. Bar is $50 \mu \mathrm{m}$. (B) Consecutive images recorded in one animal at 1 and $2 \mathrm{~h}$ after administration of 7D12-9G8-PS fluorescence (red) in tumor (green) cells. Bar is 20 $\mu \mathrm{m}$. (C) The Spearman's rank correlation coefficient for GFP fluorescence of tumor (green) and NIR fluorescence (red) of 7D12-PS (white bars) and 7D12-9G8-PS (black bars). Mean \pm SEM and n=6 and 7 respectively. Significantly difference between time before and after administration with $\mathrm{p}<0.01$ $(*), \mathrm{p}<2 \times 10^{-4}(\#)$ or $\mathrm{p}<2 \times 10^{-6}(+)$. Significant difference between 7D12-PS and 7D12-9G8-PS with $\mathrm{p}<0.003(\S)$. 
Normal tissue showed a heterogeneous fluorescence pattern. For both conjugates, fluorescence was present in close proximity of vasculature in chambers, with and without tumor (white arrows in Figure 1A). Line profiles obtained perpendicularly over larger vessels showed up to a $4 \mathrm{x}$ higher fluorescence intensity close to the vessels compared to further from the vessel (data not shown). In order to determine the localization of the fluorescence more carefully, tissue was harvested at 1 or $24 \mathrm{~h}$ after administration of the NB-PS, sectioned and stained for endothelial cells (CD31 fluorescent detection). Ex vivo fluorescence microscopy showed high fluorescence intensities for both NB-PS in normal tissue associated with the endothelial cells of (larger) blood vessels (Figure 3). 

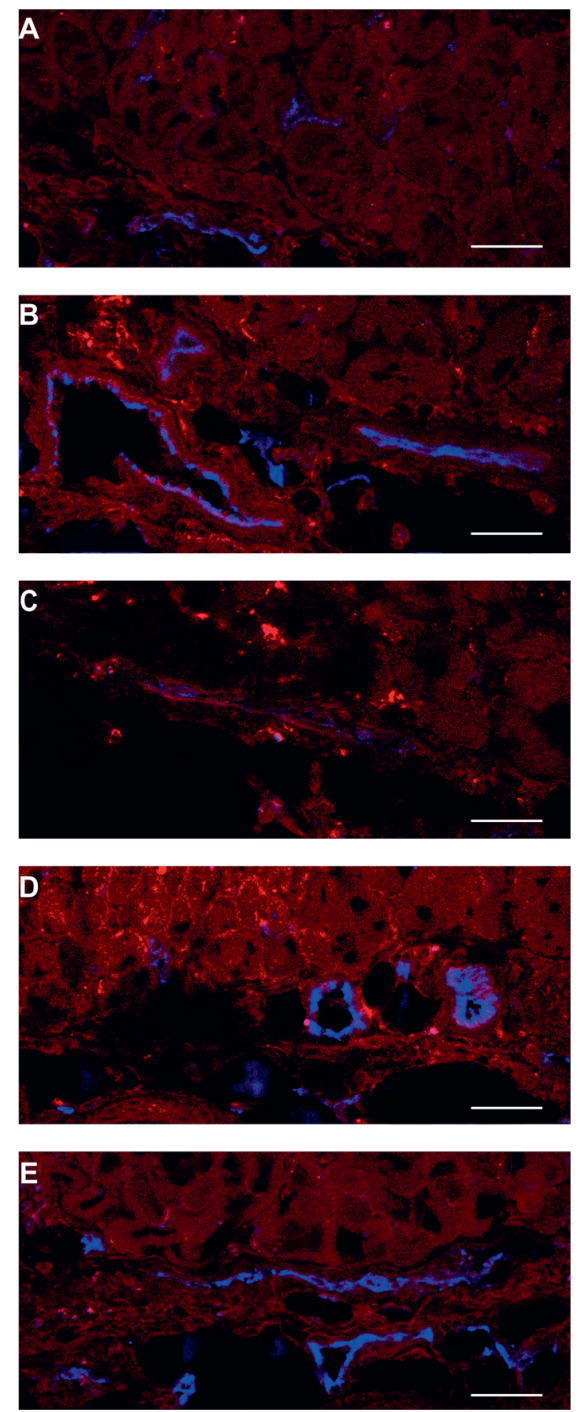

\section{Responses to nanobody targeted PDT}

Since the acute response to PDT is a complex mixture of cellular and vascular responses, we investigated both using different techniques. Initial tumor cell responses were assessed by observing changes in the GFP signal using intravital imaging. Vascular responses were investigated with intravital fluorescence imaging for vascular flow and leakage (tetramethylrhodamine dextran), intravital transmission imaging for vascular architecture; and DCE-MRI for assessment of vascular perfusion. These were combined with ex vivo histology for evaluation of necrosis marker (HQ4) and of (permanent) tissue necrosis. 


\section{Tumor cellular responses}

Distinct changes to the morphology of tumor cells were observed in the GFP fluorescence images recorded in time, after illumination at $1 \mathrm{~h}$ (Figure 4). Before illumination, the GFP signal was confined to the tumor cells, with high fluorescence intensity in the cytoplasm compared to the nucleus. In light only controls, the number of GFP containing cells increased in the $24 \mathrm{~h}$ after illumination, thus reflecting tumor growth. In NB-PS treated animals that were illuminated $1 \mathrm{~h}$ after administration, the normal cellular GFP fluorescence pattern, in some cases, changed significantly. In those cases the GFP fluorescence appeared more diffuse and/or confined to circular spots of different diameters with high fluorescence intensities, suggesting tumor cell damage. This was seen in more animals treated with 7D12-9G8-PS than with 7D12-PS (10/13 fields vs 3/12 fields, respectively) and was not observed for PDT treatments $24 \mathrm{~h}$ after administration (data not shown). Fluorescence imaging of cryosections of the tissues collected $48 \mathrm{~h}$ after PDT showed similar results (Figure 4). 

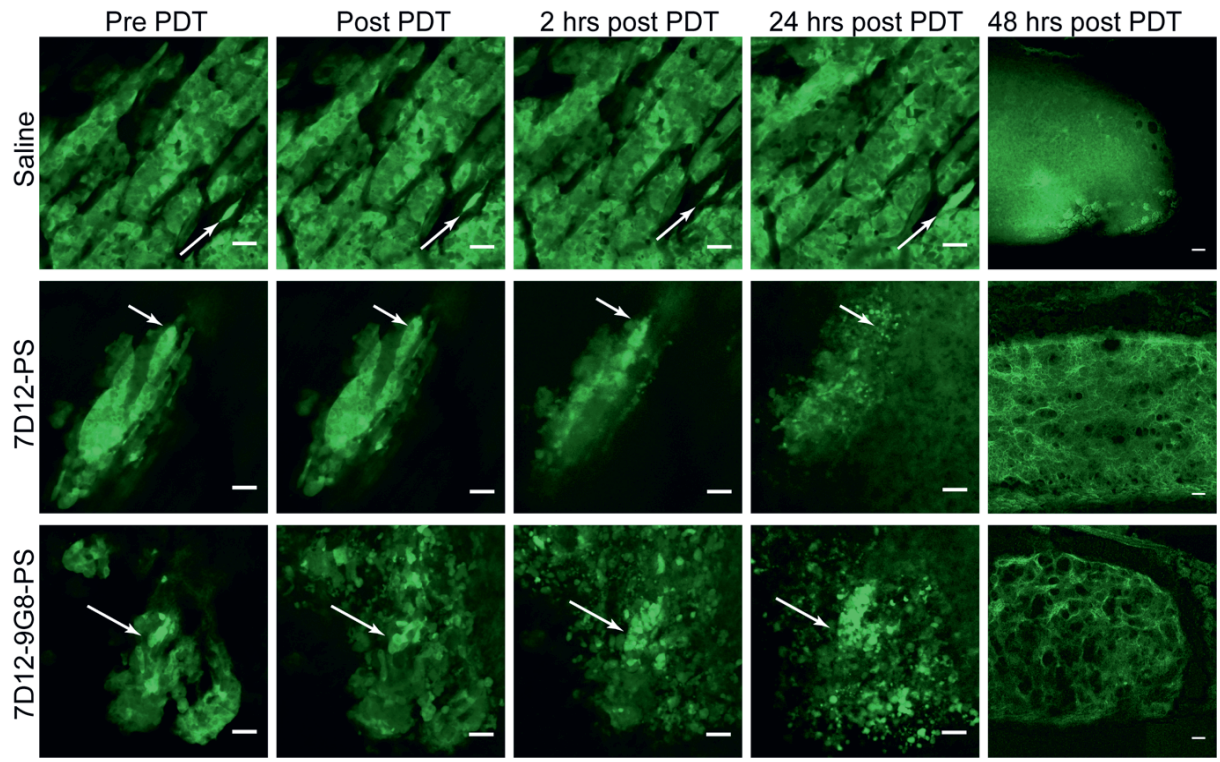

Figure 4. Examples of the effect of PDT on the morphology of tumor cells intravitally imaged in the skin-fold chamber, at $1 \mathrm{~h}$ post administration of saline (top), 7D12-PS (middle) and 7D12-9G8-PS (bottom) up to $24 \mathrm{~h}$ after PDT. Arrows point to the same cell cluster in every image for each group. The last column displays the GFP fluorescence images of cryosections obtained $48 \mathrm{~h}$ after PDT. Bar $50 \mu \mathrm{m}$.

\section{Vascular responses}

Vascular leakage of tumor and normal tissue vessels was observed for both NBPS treated groups. But there was no clear relationship between the number of tumors that showed leakage and the conjugate used or the time at which the light was delivered (Table 2). The proportional area of tumor that showed either lack of flow or reduced flow $2 \mathrm{~h}$ post PDT was similar for both conjugates when light was applied $1 \mathrm{~h}$ after administration (Figure 5). The severity of the vascular response decreased significantly when the light was delivered at $24 \mathrm{~h}$ after administration, as the size of the area with lack of flow decreased from $51 \%$ to $8 \%$ with $\mathrm{p}=0.029$ for $7 \mathrm{D} 12$-PS and from $55 \%$ to $10 \%$ with $\mathrm{p}=0.033$ for 7D12-9G8-PS. Comparing the two conjugates, a larger area of tumor showed reduced flow $2 \mathrm{~h}$ post illumination at $24 \mathrm{~h}$ after administration of 7D12-PS compared to 7D12-9G8PS (Figure 5). 
Table 2. Number of animals showing vascular leakage in tumor and normal tissue $2 \mathrm{~h}$ post PDT using either 7D12-PS or 7D12-9G8-PS and illumination at either 1 or $24 \mathrm{~h}$ after administration.

\begin{tabular}{ccccc} 
& \multicolumn{2}{c}{ Tumor } & \multicolumn{2}{c}{ Normal tissue close to tumor } \\
\cline { 2 - 5 } Conjugate & $1 \mathrm{~h}$ & $24 \mathrm{~h}$ & $1 \mathrm{~h}$ & $24 \mathrm{~h}$ \\
\hline 7D12-PS & $4 / 6$ & $2 / 6$ & $1 / 3 * 2$ & $3 / 4 * 1$ \\
\hline 7D12-9G8-PS & $3 / 8$ & $4 / 6$ & $5 / 6$ & $3 / 5$
\end{tabular}

* number of animals not scored due to lack of flow
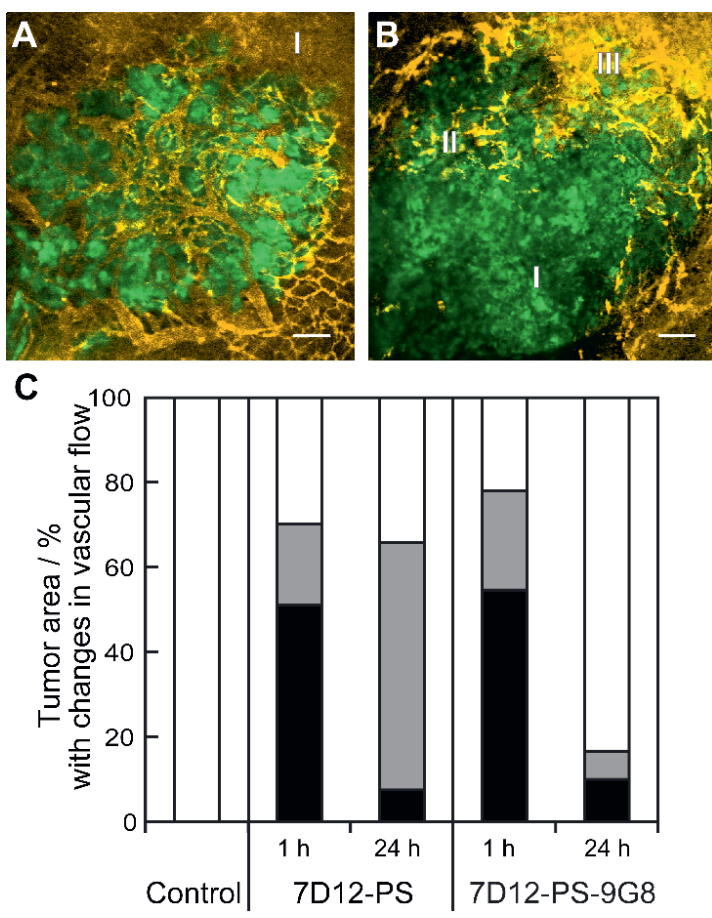

Figure 5. Representative images showing the different tumor vascular responses observed $2 \mathrm{~h}$ after PDT recorded using GFP fluorescence (green) and tertramethylrhodamine dextran fluorescence (yellow). Bar is $100 \mu \mathrm{m}$. (A) Normal blood flow in the tumor and leakage (L) just outside the tumor. (B) Tumor with an area of no flow (I), reduced flow (II) and normal flow with leakage (III). (C) Qualitative analysis of vascular flow in tumor determined $2 \mathrm{~h}$ after PDT using 7D12PS or 7D12-9G8-PS and illuminated either at 1 or $24 \mathrm{~h}$ post administration. The relative area in tumor that showed either no flow (black), reduced flow (gray) or normal flow (white) was determined and averaged in 6 animals.

DCE-MRI imaging of the solid tumors after 7D12-PS mediated PDT showed tumor non-enhancement values that were in agreement with the proportion of tumor showing lack of flow in the skin-fold chambers (Figure 6A-B). Directly after and $24 \mathrm{~h}$ after 7D12-PS mediated PDT, $42.8 \pm 22.6 \%$ and $61.9 \pm 21.4 \%$ of the tumor showed nonenhancement, i.e., loss of contrast agent uptake, which was significantly increased compared to baseline ( $\mathrm{p}=0.01$ and $4.6810-4$, respectively). In agreement with the loss of 
contrast agent, uptake in tumors showed negligible $\mathrm{K}^{\text {trans }}$ values throughout the whole tumor at both time points (Figure 6C-D). The remaining pixels with residual contrast enhancement mostly showed lower $\mathrm{K}^{\text {trans }}$ values compared to untreated tumor tissue. Most parts of the underlying muscle showed a decrease in $\mathrm{K}^{\text {trans }}$ immediately and $24 \mathrm{~h}$ after PDT, suggesting impaired microvascular perfusion, although this was not statistically significant ( $\mathrm{p}=0.13$ and 0.24 respectively).

A
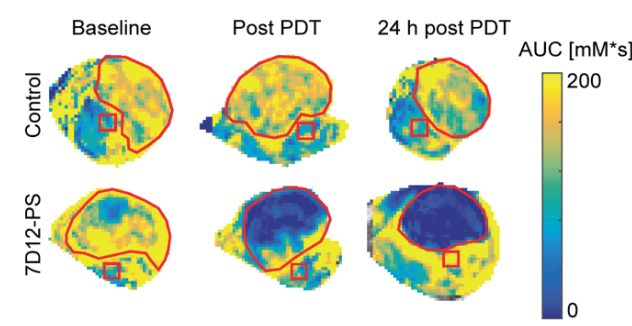

C
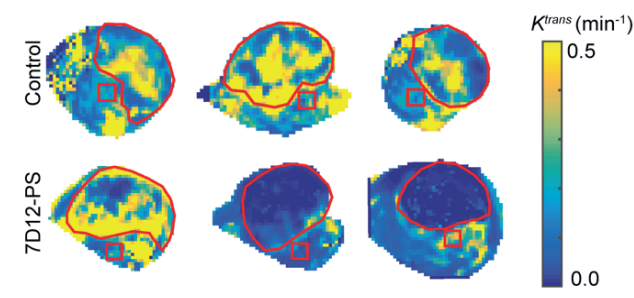

B
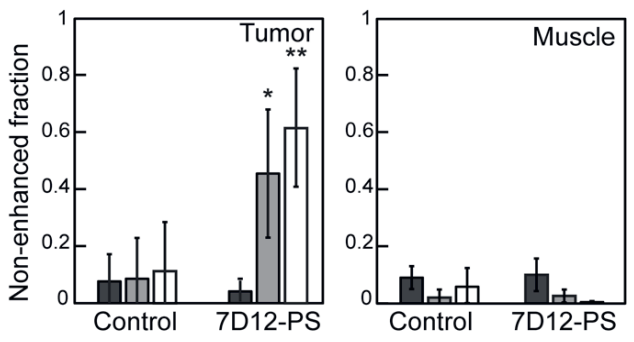

D

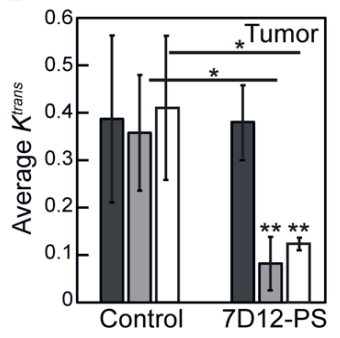

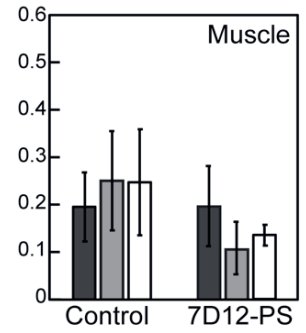

Figure 6. (A) Representative AUC maps of a control and 7D12-PS mediated PDT treated animals recorded at baseline, post PDT and $24 \mathrm{~h}$ post PDT. The red contours outline the tumor, the red squares represent muscle ROI. (B) Group averages of the non-enhanced fraction pre PDT (black), post PDT (grey) and $24 \mathrm{~h}$ post PDT (white) in enhanced tumor and muscle ROI's. Significant

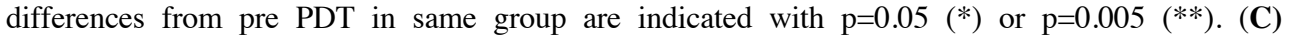
Representative $K^{\text {trans }}$ maps of a control and 7D12-PS mediated PDT treated animal recorded at baseline, post PDT and $24 \mathrm{~h}$ post PDT. The red contours outline the tumor, the red squares represent muscle ROI. (D) Group averages of the mean $K^{\text {trans }}$ pre PDT (black), post PDT (gray) and 24 h post PDT (white) in enhanced tumor pixels and muscle ROI's. Significant differences from pre PDT from same group or between groups are indicated with $\mathrm{p}=0.05(*)$ or $\mathrm{p}=0.005(* *)$.

The normal skin vasculature showed constriction of the larger arterioles and venules, and also changed vascular architecture post NB-PS mediated PDT. Comparing the two conjugates, slight differences were observed, 1 animal showed more severe constriction $2 \mathrm{~h}$ post PDT using 7D12-PS compared to 7D12-9G8-PS but this was reversed $48 \mathrm{~h}$ after PDT. Illumination at $24 \mathrm{~h}$ after administration resulted in slightly more vascular 
constriction at 24 and $48 \mathrm{~h}$ post PDT for 7D12-PS compared to 7D12-9G8-PS. Overall, these effects were not significantly different for the two conjugates, only more severe for illumination at $1 \mathrm{~h}$ compared to $24 \mathrm{~h}$ after administration (Figure 7).
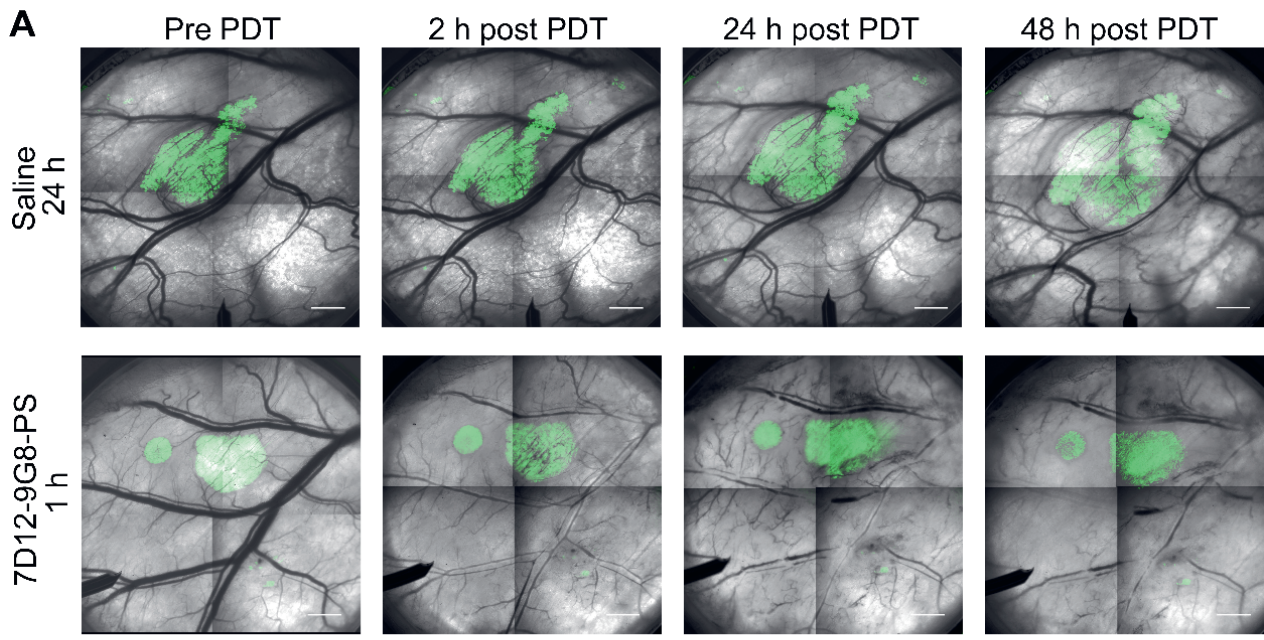

B
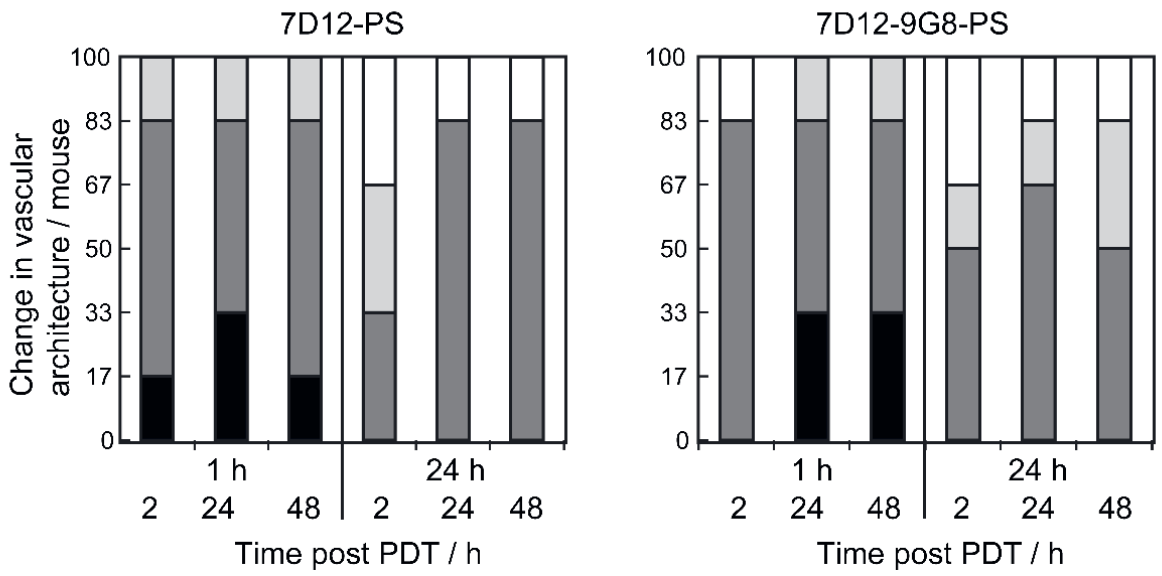

Figure 7. (A) Representative images of the changes in vascular architecture in the skin-fold chamber tissue collected longitudinally before and in time after NB-PS mediated PDT delivered $1 \mathrm{~h}$ post administration. Bar $=1000 \mu \mathrm{m}$. (B) Severity of the change in vascular architecture in normal tissue per mouse scored at different time points after PDT at 1 or $24 \mathrm{~h}$ using 7D12-PS or 7D12-9G8-PS. No change in vascular lumen and normal blood flow was scored white (-), capillary shutdown and or small changes in vascular lumen (light grey, + ), severe changes is vascular lumen but blood flow observed (dark grey, ++ ) and severe changes in vascular lumen and no flow observed (black, +++ ). 


\section{Tumor and normal tissue viability and necrosis}

The PDT induced vascular responses and edema significantly changed the tissue optical properties of tissues in the window chamber, rendering intravital imaging of the necrosis avid agent HQ4 $48 \mathrm{~h}$ after PDT impossible. Therefore, imaging of HQ4 was performed on cryosections of excised tissues. More fluorescence of HQ4 was observed in tumors treated with NB targeted PDT compared to the light only controls (Figure 8). While it is unknown if there is a linear relationship between HQ4 fluorescence intensity and necrosis, the data suggests more necrosis for illumination at $1 \mathrm{~h}$ after administration compared to $24 \mathrm{~h}$ after administration.

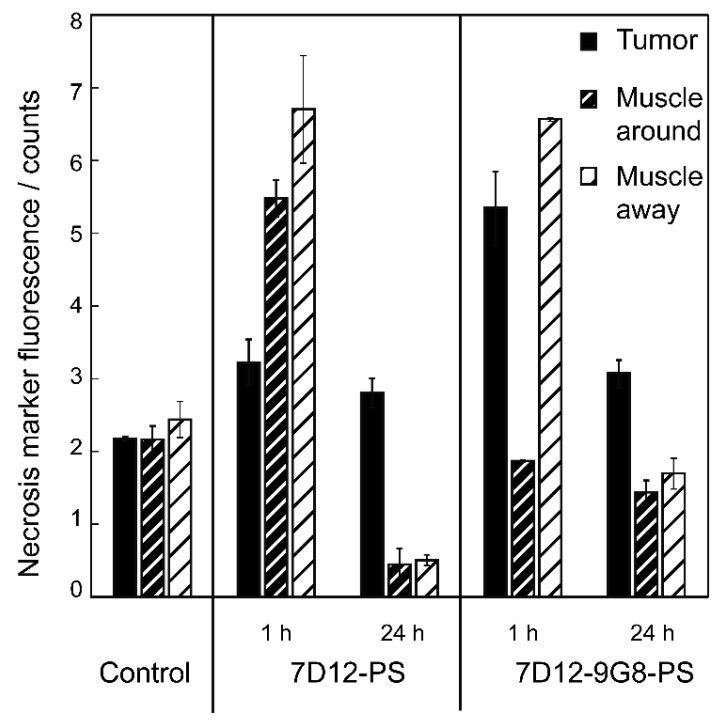

Figure 8. Fluorescence intensity of the necrosis marker HQ4 in cryosections of the skin-fold chamber collected $48 \mathrm{~h}$ after PDT at $1 \mathrm{~h}$ post administration of physiological saline, 7D12-PS, 7D129G8-PS, and after PDT at $24 \mathrm{~h}$ post administration of 7D12-PS and 7D129G8-PS ROI's were drawn around tumor (black bar) and sub cutaneous musculature around and away from tumor (fat or thin dashed bar) respectively. Weighted mean $\pm \mathrm{SD}$, $\mathrm{n}=1-6$ animals per group and $2-3$ sections per animal.

H\&E staining of the skin-fold chamber tissues also showed more tumor necrosis for illumination at $1 \mathrm{~h}$ compared to $24 \mathrm{~h}$ post administration (Figure 9A-D). PDT at $1 \mathrm{~h}$ after administration induced 88 or $80 \%$ necrosis in tumors compared to 30 or $0 \%$ at $24 \mathrm{~h}$ for 7D12-PS and 7D12-9G8-PS, respectively. Complementary to this, a similar average necrotic tumor fraction was determined in H\&E sections of the solid tumor model for treatment with 7D12-PS mediated PDT at $2 \mathrm{~h}(89 \pm 9 \%$, compared to $23 \pm 20 \%$ in light only controls). Fluorescence imaging of Hoechst 33342 in adjacent sections showed colocalization of those areas with the non-perfused areas $(74 \pm 17 \%$ and $23 \pm 33 \%$, respectively). Tumors treated with 7D12-PS mediated PDT hardly showed any uptake of 
Hoechst 33342 and fewer blood vessels were detected. Blood vessels had weak staining and indistinct morphology, possibly due to disruption and closed lumina (Figure 9E-H).
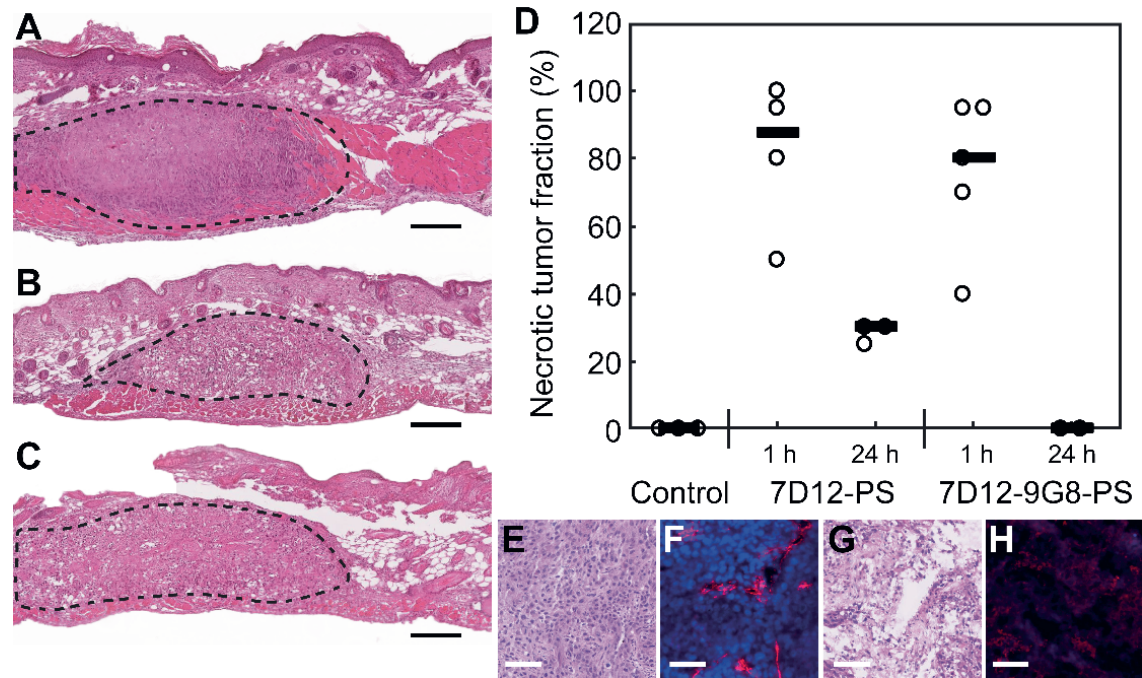

Figure 9. Example of H\&E stained sections of the skin-fold chamber $48 \mathrm{~h}$ after PDT at $1 \mathrm{~h}$ post administration of (A) physiological saline, (B) 7D12-PS or (C) 7D12-9G8-PS. Tumor is outlined with dashed lines. Black bar is $200 \mu \mathrm{m}$. (D) Individual percentages of tumor necrosis and the median per treatment. Representative examples of (E,G) H\&E bright-field images and (F,H) CD31 \& Hoechst 33342 fluorescence images of (E,F) control and (G,H) 7D12-PS mediated PDT treated solid tumors. CD31 is displayed in red and Hoechst in blue. White bar is $100 \mu \mathrm{m}$.

Concerning normal tissues, damage to these in the skin-fold chamber model was similar for both NB-PS conjugates used (Table 3). PDT delivered $1 \mathrm{~h}$ after administration resulted in severe damage to the normal tissues close to tumor. Away from the tumor, the damage was mild. No damage to normal tissues was observed when PDT was performed $24 \mathrm{~h}$ after administration. Also, the skin-fold chambers without tumor showed no damage to normal tissues $48 \mathrm{~h}$ after PDT at either 1 or $24 \mathrm{~h}$ post administration ( $\mathrm{n}=1$ per group). 
Table 3. Degree of tissue damage scored in H\&E sections of the skin-fold chamber collected $48 \mathrm{~h}$ after PDT with 2 anti-EGFR nanobody-photosensitizer conjugates. Mean of 3 to 5 animals per group.

\begin{tabular}{|c|c|c|c|c|c|c|}
\hline & \multirow[t]{2}{*}{ Control } & \multicolumn{2}{|c|}{ 7D12-PS } & \multicolumn{2}{|c|}{ 7D12-9G8-PS } & \multirow{2}{*}{$\begin{array}{c}\text { No tumor } \\
\text { All NB- } \\
\text { PS } \\
1 \text { or } 24 \mathrm{~h}\end{array}$} \\
\hline & & $1 \mathrm{~h}$ & $24 \mathrm{~h}$ & $1 \mathrm{~h}$ & $24 \mathrm{~h}$ & \\
\hline $\begin{array}{l}\text { Damage to } \\
\text { epithelium }\end{array}$ & - & +++ & + & +++ & - & - \\
\hline $\begin{array}{l}\text { Damage to muscle } \\
\text { cells } \\
\text { around tumor }\end{array}$ & - & +++ & - & $+++/++$ & - & - \\
\hline $\begin{array}{l}\text { Damage to muscle } \\
\text { cells } \\
\text { away from tumor }\end{array}$ & - & ++ & - & $++/+$ & - & - \\
\hline $\begin{array}{l}\text { Damage to blood } \\
\text { vessels } \\
\text { around tumor }\end{array}$ & - & +++ & - & +++ & - & - \\
\hline $\begin{array}{l}\text { Damage to blood } \\
\text { vessels } \\
\text { away from tumor }\end{array}$ & - & ++ & - & $++/+$ & - & - \\
\hline Neutrophils & Few & Some & Some & Few & Few & - \\
\hline
\end{tabular}

\section{Discussion}

The present study was designed to investigate the biodistribution of two NB-PS, the cellular responses to and the vascular effects of EGFR-targeted PDT in vivo using intravital microscopy and DCE-MRI, complemented with ex vivo analysis of necrosis.

The overall fluorescence kinetics determined with intravital microscopy were similar to those we have previously reported for the orthotopic mouse tongue model using quantitative fluorescence fiber optic spectroscopy [16]. In both cases, we have shown more NB-PS fluorescence in tumor than in normal tissue, and that the mean intensity was higher after administration of biparatopic 7D12-9G8-PS, compared to monovalent 7D12-PS. Intravital microscopy imaging however, revealed a heterogeneous fluorescence pattern in tumor that was different for the two conjugates. 7D12-9G8-PS showed a membrane bound fluorescence pattern in tumor, which was not observed for 7D12-PS. The reduced dissociation kinetics of 7D12-9G8-PS compared to 7D12-PS and/or the induced 
internalization of 7D12-9G8-PS might play a role in the observed higher fluorescence intensity and the more membrane bound fluorescence pattern for 7D12-9G8-PS [15] Previously, the highest fluorescence intensity of 7D12-PS was detected in tumor $30 \mathrm{~min}$ after administration with a clear decrease thereafter, whereas for 7D12-9G8-PS the fluorescence intensity remained high for at least $4 \mathrm{~h}$ after administration [16]. 7D12-PS may be already dissociating from the receptor at early time points after administration and therefore showing less membrane localization at 1 and $2 \mathrm{~h}$ after administration. Internalization of 7D12-9G8-PS has been shown in vitro and was observed only once intravitally, possibly because of the many factors that influence the effective resolution of intravital imaging [24].

Consistent with the visual observation, colocalization analysis between GFP signal and photosensitizer fluorescence showed significantly higher Spearman's rank correlation coefficients, compared to the background, for both conjugates, suggesting tumor specific binding in time after administration which was significantly higher for 7D12-9G8-PS compared to 7D12-PS.While this study was focused on targeted PDT, targeted NB-PS conjugates have the potential to be used in fluorescence image guided surgery $[51,61-63]$. Complete tumor resection relies on the surgeon's ability to differentiate between malignant and benign tissue but the infiltrative nature of cancerous tissue may hinder this. Fluorescence image guided surgery may assist a surgeon in delineating the cancerous tissue in the surgical field. NB-PS conjugates with their small size, high penetration and targeting characteristics, combined with fast clearance when unbound, could be excellent candidates for fluorescence image guided surgery. Clearance should be rapid enough to not have side effects of being photosensitive and long enough to last through the surgical procedure. Comparing the two conjugates in this study, 7D12-9G8-PS seems to be a good candidate, because of the high correlation coefficient between 7D129G8-PS fluorescence and tumor cells and the prolonged increased tumor fluorescence intensity in time after administration.

In normal tissue, a similar heterogeneous fluorescence distribution was found for both NB-PS, each showing fluorescence in the fascia and endothelial tissue up to $24 \mathrm{~h}$ after administration. We have previously detected NB-PS fluorescence in normal mouse tissues, such as tongue and skin, using fiber optic spectroscopy [16]. But fiber optics spectroscopy reveals only information in intensity and not on cellular distribution. In the present 
intravital microscopy study, we observe that there are inhomogeneously distributed high intensities in the fascia and (close to) endothelial cells. Based on our current understanding of EGFR-targeted NB-PS, it is unclear why conjugates are present in normal tissue of the skin-fold chamber.

PDT induced damage is, in general, the result of a complex mixture of direct tumor cell damage, the influence of the (tumor) vascular responses and the immunological responses to these effects [1]. In the previous study, we showed necrosis of the tumor combined with loss of CD31 and an increased presence of neutrophils after NB-PS mediated PDT [16]. Our results here show membrane-localized fluorescence in tumor cells and also endothelial cell-associated fluorescence in the vasculature, thus considering the short diffusion distance of singlet oxygen, these are likely the primary sites of photodamage [27].

To assess the effects of NB-targeted PDT, first the morphology of the tumor cells was visualized using GFP fluorescence imaging. In some cases, the cellular GFP fluorescence pattern showed diffuse fluorescence and/or high fluorescence intensities confined to circular spots of different diameters, as early as $2 \mathrm{~h}$ after PDT, suggesting the degradation of tumor cells. In agreement with the difference in fluorescence intensity, and probably also with the differences in localization, more animals treated with 7D12-9G8-PS mediated PDT at $1 \mathrm{~h}$ post administration showed degradation of tumor cells compared to 7D12-PS. Based on these results, a difference in PDT-induced necrosis between the two conjugates might be expected but was not observed to be statistically significant. Ex vivo fluorescence imaging of HQ4, a necrosis avid cyanine probe [41,42], showed more tumor necrosis after 7D12-9G8-PS compared to 7D12-PS mediated PDT when light was applied $1 \mathrm{~h}$ after administration. However, while it is unknown if there is a linear relationship between HQ4 fluorescence intensity and necrosis, little can be said about the significance of this result. Histological evaluation of tumor necrosis showed slightly, but not significantly more, necrosis post 7D12-PS compared to 7D12-9G8-PS mediated PDT.

Vascular responses are a known contributor to the overall PDT response and those were broadly similar for both conjugates. Tumor vascular leakage was observed in more than $50 \%$ of the treated tumors within $2 \mathrm{~h}$ after PDT (Table 2). In our previous study we showed damage to the vasculature by histological evaluation $24 \mathrm{~h}$ post PDT [16]. In the present study we did not find large differences in the magnitude of vascular responses for 
each conjugate. We did not investigate the effects of different NB-PS doses on the vasculature and overall response to PDT. It is likely that higher or lower doses of NB-PS will lead to different vascular responses in tumor and normal tissue depending also on the oxygen availability and the light applied.

The vascular effect of PDT could be utilized to enhance the EPR effect, in combined therapies with nanosized drug delivery systems [29-31]. Employing a NB specifically designed to target the tumor vasculature could possibly increase this response even further. However, this should be investigated with caution, as tumor vascular stasis was also observed in 51-54\% of the tumor areas $2 \mathrm{~h}$ after PDT, detected with rhodamine dextran fluorescence. This decreased perfusion was also confirmed through DCE-MRI of the subcutaneous tumor model, $1 \mathrm{~h}$ after 7D12-PS mediated PDT. This illustrates that any combination with PDT needs to be carefully investigated so that there is an optimal enhancement of the EPR effect. Tumor cell targeted, endothelial cell targeted, and conventional PDT might affect the vasculature differently. Therefore, intravital microscopic detailed studies, such as the one we present here, are critical to investigate a possible time window in which leakage could be exploited, before perfusion is compromised.

In line with the fluorescence localization of the conjugates, normal tissue showed significant changes in the vascular architecture of skin-fold chambers after PDT that were more severe for PDT at $1 \mathrm{~h}$ compared to $24 \mathrm{~h}$. Histological examination revealed abundant damage to the subcutaneous musculature and fascia after PDT at $1 \mathrm{~h}$ but not at $24 \mathrm{~h}$ (Table 3 ). It would be interesting to investigate the vascular effects with a slightly longer time between administration and illumination, but significantly shorter than $24 \mathrm{~h}$, or with lower doses of the NB-PS conjugates. Perhaps then less vascular damage in normal surrounding tissues would be observed, though with the risk of decreased tumor damage, which after all is the main goal. Skin-fold chambers not transplanted with tumors showed similar changes in the vascular architecture (detected with intravital microscopy) but no damage to the musculature or to blood vessels (detected in tissue sections). This suggests that the vascular effects in normal tissue are transient when no tumor is present, and that, when tumor is present, the responding tumor contributes to the damage of surrounding normal tissue.

PDT at $24 \mathrm{~h}$ post administration was investigated in an attempt to utilize the higher tumor to normal ratio. Increasing the time between administration and illumination 
from $1 \mathrm{~h}$ to $24 \mathrm{~h}$ resulted in less vascular responses in tumor and normal tissue (much smaller tumor area showed lack of flow and less changes in normal vascular architecture) but also significantly less tumor response. At $24 \mathrm{~h}$ after administration, 7D12-9G8-PS still showed significant, although lower, colocalization of photosensitizer fluorescence with GFP suggesting that tumor cells would respond to PDT. However, the GFP fluorescence pattern of tumor cells after PDT had not changed, suggesting lack of tumor cell degradation. Indeed, histological evaluation showed a much lower necrotic tumor fraction after PDT at $24 \mathrm{~h}$. In addition, HQ4 fluorescence also showed decreased necrosis in tumor and normal tissue after PDT at $24 \mathrm{~h}$ compared to $1 \mathrm{~h}$. Although the TNR is much higher at $24 \mathrm{~h}$ after administration, the fluorescence intensity in the tumor was significantly lower. The current data clearly show that a high TNR is not the only factor in an effective treatment scheme: the concentration of NB-PS in the tumor is critically important. This result is in agreement with results from our previous study in the oral cavity [16].

The data presented here confirm that the overall acute response to EGFR-targeted NB-PS mediated PDT is not just a tumor cell specific response but a complex mixture of tumor cell responses and vascular effects. From a clinical perspective the combination of direct tumor cell death and tumor vascular damage is likely to be necessary for a maximally effective treatment.

In summary, we have obtained insights with unprecedented detail for targeted PDT, which are significant at present, since EGFR-targeted PDT using antibodies as carriers is currently being tested in phase I/II clinical trials [64]. NB-PS conjugates 7D12PS and 7D12-9G8-PS targeting EGFR showed significant tumor localization in vivo, which was higher for 7D12-9G8-PS. The significant higher tumor colocalization combined with the prolonged fluorescence intensity in time after administration makes 7D12-9G8-PS a good candidate for fluorescence image guided surgery. Illumination at $1 \mathrm{~h}$ after administration of either conjugates leads to significant tumor necrosis. Despite the difference in fluorescence intensity, no significant difference was observed in the overall acute tissue response; both EGFR-targeted NB-PS mediated PDT treatments lead to similar amount of tumor necrosis 2 days post treatment and no large differences in vascular responses. Growth delay studies are now needed to show the long-term efficacy of nanobody-targeted PDT. 


\section{Acknowledgements}

The authors thank Gert-Jan Kremers, Alex Niggs and Gert van Cappellen of the Optical Imaging Centre of the Erasmus MC for their support on confocal microscopy imaging. We also thank Katerina Xenaki for her assistance preparing the conjugates. This project has received funding from the European Research Council (ERC) under the European Union's Horizon 2020 research and innovation program (grant agreement No 677582).

\section{References}

1. van Straten D, Mashayekhi V, de Bruijn HS, Oliveira S, Robinson DJ. Oncologic photodynamic therapy: basic principles, current clinical status and future directions. Cancers. 2017; 9: 19.

2. Agostinis P, Berg K, Cengel KA, Foster TH, Girotti AW, Gollnick SO, et al. Photodynamic therapy of cancer: An update CA Cancer J Clin. 2011; 61: 250-81.

3. Civantos FJ, Karakullukcu B, Biel M, Silver CE, Rinaldo A, Saba NF, et al. A Review of Photodynamic Therapy for Neoplasms of the Head and Neck. Adv Ther. 2018; 35: 324-40.

4. Lutje S, Heskamp S, Franssen GM, Frielink C, Kip A, Hekman M, et al. Development and characterization of a theranostic multimodal anti-PSMA targeting agent for imaging, surgical guidance, and targeted photodynamic therapy of PSMA-expressing tumors. Theranostics. 2019; 9: 2924-38.

5. Sato K, Nagaya T, Choyke PL, Kobayashi H. Near infrared photoimmunotherapy in the treatment of pleural disseminated NSCLC: preclinical experience. Theranostics. 2015; 5: 698-709.

6. Grandis JR, Tweardy DJ. Elevated levels of transforming growth factor alpha and epidermal growth factor receptor messenger RNA are early markers of carcinogenesis in head and neck cancer. Cancer Res. 1993; 53: 3579-84.

7. Lim CK, Heo J, Shin S, Jeong K, Seo YH, Jang WD, et al. Nanophotosensitizers toward advanced photodynamic therapy of cancer. Cancer Lett. 2013; 334: 176-87.

8. Kaščáková S HL, De Bruijn HS, Ye Y, Achilefu S, van der Wansem K, van der Ploeg-van den Heuvel A, van Koetsveld PM, Brugts MP, van der Lelij AJ, Sterenborg HL, Ten Hagen TL, Robinson DJ, van Hagen MP. Somatostatin analogues for receptor targeted photodynamic therapy. PLos One. 2014; 9: e104448.

9. Abrahamse H, Hamblin MR. New photosensitizers for phototdynamic therapy. Biochem J. 2016; 473: 347-64.

10. Derycke ASL, de Witte PAM. Liposomes for photodynamic therapy. Adv Drug Deliv Rev. 2004; 56: $17-20$.

11. van Dongen GA, Visser GW, Vrouenraets MB. Photosensitizer-antibody conjugates for detection and therapy of cancer. Adv Drug Deliv Rev 2004; 56: 31-52.

12. Sato K, Watanabe R, Hanaoka H, Harada T, Nakajima T, Kim I, et al. Photoimmunotherapy: Comparative effectiveness of two monoclonal antibodies targeting the epidermal growth factor receptor. Mol Oncol. 2014; 8: 620-32.

13. Mitsunaga M, Ogawa M, Kosaka N, Rosenblum LT, Choyke PL, Kobayashi H. Cancer cellselective in vivo near infrared photoimmunotherapy targeting specific membrane molecules. Nat Med. 2011; 17: 1685-91.

14. Mou J, Lin T, Huang F, Shi J, Chen H. A New Green Titania with Enhanced NIR Absorption for Mitochondria-Targeted Cancer Therapy. Theranostics. 2017; 7: 1531-42.

15. Heukers R, van Bergen en Henegouwen PM, Oliveira S. Nanobody-photosensitizer conjugates for targeted photodynamic therapy. Nanomedicine. 2014; 10: 1441-51.

16. van Driel PBAA, Boonstra MC, Slooter M, Heukers R, Stammes MA, Snoeks TJA, et al. EGFR targeted nanobody-photosensitizer conjugates for photodynamic therapy in a pre-clinical model of head and neck cancer. J Control Release. 2016; 229: 93-105. 
17. Heukers R, Mashayekhi V, Ramirez-Escudero M, de Haard H, Verrips TC, van Bergen en Henegouwen PMP, et al. VHH-Photosensitizer Conjugates for Targeted Photodynamic Therapy of Met-Overexpressing Tumor Cells. Antibodies. 2019; 8: 26.

18. De Groof TWM, Mashayekhi V, Fan TS, Bergkamp ND, Sastre Toraño J, van Senten JR, et al. Nanobody-Targeted Photodynamic Therapy Selectively Kills Viral GPCR-Expressing Glioblastoma Cells. Mol Pharm. 2019; 16: 3145-56.

19. Hamers-Casterman C, Atarhouch T, Muyldermans S, Robinson G, Hamers C, Songa EB, et al. Naturally occurring antibodies devoid of light chains. Nature. 1993; 363: 446-8.

20. Muyldermans S, Atarhouch T, Saldanha J, Barbosa JA, Hamers R. Sequence and structure of VH domain from naturally occurring camel heavy chain immunoglobulins lacking light chains. Protein Eng 1994; 7: 1129-35.

21. Muyldermans S. Nanobodies: natural single-domain antibodies. Annu Rev Biochem. 2013; 82: 775-97.

22. Oliveira S, Heukers R, Sornkom J, Kok RJ, van Bergen En Henegouwen PM. Targeting tumors with nanobodies for cancer imaging and therapy. J Control Release. 2013; 172: 607-17.

23. Roovers RC, Vosjan MJ, Laeremans T, el Khoulati R, de Bruin RC, Ferguson KM, et al. A biparatopic anti-EGFR nanobody efficiently inhibits solid tumour growth. Int J Cancer. 2011; 129: 2013-24.

24. Heukers R, Vermeulen JF, Fereidouni F, Bader AN, Voortman J, Roovers RC, et al. Endocytosis of EGFR requires its kinase activity and N-terminal transmembrane dimerization motif. J Cell Sci. 2013; 126: 4900-12.

25. Peng X, Draney DR, Volcheck WM, Bashford GR, Lamb DT, Grone DL, et al. Phthalocyanine dye as an extremely photostable and highly fluorescent near-infrared labeling reagent In: Achilefu S, Bornhop D, Raghavachari R, editors. Optical Molecular Probes for Biomedical Applications. San Jose: Elsevier B.V.; 2006. p. 60970E.

26. Olson MT, Ly QP, Mohs AM. Fluorescence Guidance in Surgical Oncology: Challenges, Opportunities, and Translation. Mol Imaging Biol. 2019; 21: 200-18.

27. Hatz S, Poulsen L, Ogilby PR. Time-resolved singlet oxygen phosphorescence measurements from photosensitized experiments in single cells: Effects of oxygen diffusion and oxygen concentration. Photochem Photobiol. 2008; 84: 1284-90.

28. Zhen Z, Tang W, Chuang YJ, Todd T, Zhang W, Lin X, et al. Tumor vasculature targeted photodynamic therapy for enhanced delivery of nanoparticles. ACS Nano. 2014; 8: 6004-13.

29. Snyder JW, Greco WR, Bellnier DA, Vaughan L, Henderson BW. Photodynamic therapy: a means to enhanced drug delivery to tumors. Cancer Res. 2003; 63: 8126-31.

30. Gao W, Wang Z, Lv L, Yin D, Chen D, Han Z, et al. Photodynamic Therapy Induced Enhancement of Tumor Vasculature Permeability Using an Upconversion Nanoconstruct for Improved Intratumoral Nanoparticle Delivery in Deep Tissues. Theranostics. 2016; 6: 1131-44.

31. Sano K, Nakajima T, Choyke PL, Kobayashi H. Markedly enhanced permeability and retention effects induced by photo-immunotherapy of tumors. ACS Nano. 2013; 7: 717-24.

32. van Leengoed HL, Cuomo V, Versteeg AA, van der Veen N, Jori G, Star WM. In vivo fluorescence and photodynamic activity of zinc phthalocyanine administered in liposomes. Br J Cancer. 1994; 69: 840-5.

33. de Bruijn HS, Brooks S, van der Ploeg-van den Heuvel A, Ten Hagen TLM, de Haas ERM, J. RD. Light Fractionation Significantly Increases the Efficacy of Photodynamic Therapy Using BF200 ALA in Normal Mouse Skin. Plos One. 2016; 11: e0148850.

34. Dicheva BM, Seynhaeve AL, Soulie T, Eggermont AM, Ten Hagen TL, Koning GA. Pharmacokinetics, Tissue Distribution and Therapeutic Effect of Cationic Thermosensitive Liposomal Doxorubicin Upon Mild Hyperthermia. Pharm Res. 2016; 33: 627-38.

35. van Leengoed HL, van der Veen N, Versteeg AA, Ouellet R, van Lier JE, Star WM. In vivo photodynamic effects of phthalocyanines in a skin-fold observation chamber model: role of central metal ion and degree of sulfonation. Photochem Photobiol. 1993; 58: 575-80.

36. van Leengoed HL, van der Veen N, Versteeg AA, Ouellet R, van Lier JE, Star WM. In vivo fluorescence kinetics of phthalocyanines in a skin-fold observation chamber model: role of central metal ion and degree of sulfonation. Photochem Photobiol. 1993; 58: 233-7. 
37. Middelburg TA, de Bruijn HS, Tettero L, van der Ploeg van den Heuvel A, Neumann HA, de Haas ER, et al. Topical hexylaminolevulinate and aminolevulinic acid photodynamic therapy: complete arteriole vasoconstriction occurs frequently and depends on protoporphyrin IX concentration in vessel wall. J Photochem Photobiol B. 2013; 126: 26-32.

38. van der Veen N, van Leengoed HL, Star WM. In vivo fluorescence kinetics and photodynamic therapy using 5-aminolaevulinic acid-induced porphyrin: increased damage after multiple irradiations. Br J Cancer. 1994; 70: 867-72.

39. Debefve E, Cheng C, Schaefer SC, Yan H, Ballini JP, van den Bergh H, et al. Photodynamic therapy induces selective extravasation of macromolecules: Insights using intravital microscopy. J Photochem Photobiol B. 2010; 98: 69-76.

40. Fingar VH, Wieman TJ, Wiehle SA, Cerrito PB. The role of microvascular damage in photodynamic therapy: the effect of treatment on vessel constriction, permeability, and leukocyte adhesion. Cancer Res. 1992; 52: 4914-21.

41. Stammes MA, Knol-Blankevoort VT, Cruz LJ, Feitsma HRIJ, Mezzanotte L, Cordfunke RA, et al. Pre-clinical evaluation of a cyanine-based SPECT probe for multimodal tumor necrosis imaging. Mol Imaging Biol 2016; 18: 905-15.

42. Stammes MA, Maeda A, Bu J, Scollard DA, Kulbatski I, Medeiros PJ, et al. The necrosis-avid small molecule HQ4-DTPa as a multimodal imaging agent for monitoring radiation therapyinduced tumor cell death. Front Oncol. 2016; 6: 221.

43. Dodd NJ, Moore JV, Poppitt DG, Wood B. In vivo magnetic resonance imaging of the effects of photodynamic therapy. Br J Cancer. 1989; 60: 164-7.

44. Mathews MS, Chighvinadze D, Gach HM, Uzal FA, Madsen SJ, Hirschberg H. Cerebral edema following photodynamic therapy using endogenous and exogenous photosensitizers in normal brain. Lasers Surg Med. 2011; 43: 892-900.

45. Huang Z, Haider MA, Kraft S, Chen Q, Blanc D, Wilson BC, et al. Magnetic resonance imaging correlated with the histopathological effect of Pd-bacteriopheophorbide (Tookad) photodynamic therapy on the normal canine prostate gland. Lasers Surg Med. 2006; 38: 672-81.

46. Haider MA, Davidson SR, Kale AV, Weersink RA, Evans AJ, Toi A, et al. Prostate gland: MR imaging appearance after vascular targeted photodynamic therapy with palladiumbacteriopheophorbide. Radiology. 2007; 244: 196-204.

47. Winsborrow BG, Grondey H, Savoie H, Fyfe CA, Dolphin D. Magnetic resonance imaging evaluation of photodynamic therapy-induced hemorrhagic necrosis in the murine M1 tumor model. Photochem Photobiol. 1997; 66: 847-52.

48. Kennedy SD, Szczepaniak LS, Gibson SL, Hilf R, Foster TH, Bryant RG. Quantitative MRI of Gd-DTPA uptake in tumors: response to photodynamic therapy. Magn Reson Med. 1994; 31: 292-301.

49. Schreurs TJL, Hectors SJ, Jacobs I, Grull H, Nicolay K, Strijkers GJ. Quantitative MultiParametric Magnetic Resonance Imaging of Tumor Response to Photodynamic Therapy. PLoS One. 2016; 11: e0165759.

50. Schreurs TJL, Jacobs I, Nicolay K, Prompers JJ, Strijkers GJ. Detection of treatment success after photodynamic therapy using dynamic contrast-enhanced magnetic resonance imaging. Theranostics. 2017; 7: 4643-57.

51. Oliveira S, van Dongen GA, Stigter-van Walsum M, Roovers RC, Stam JC, Mali W, et al. Rapid visualization of human tumor xenografts through optical imaging with a near-infrared fluorescent anti-epidermal growth factor receptor nanobody. Mol Imaging. 2012; 11: 33-46.

52. Schmitz KR, Bagchi A, Roovers RC, van Bergen en Henegouwen PM, Ferguson KM. Structural evaluation of EGFR inhibition mechanisms for nanobodies/VHH domains. Structure. 2013; 21: 1214-24.

53. van Driel PB, van der Vorst JR, Verbeek FP, Oliveira S, Snoeks TJ, Keereweer S, et al Intraoperative fluorescence delineation of head and neck cancer with a fluorescent anti-epidermal growth factor receptor nanobody. Int J Cancer. 2014; 134 2663-73.

54. Seynhaeve ALB, Hoving S, Schipper D, Vermeulen CE, aan de Wiel-Ambagtsheer G, van Tiel ST, et al. Tumor necrosis factor $\alpha$ mediates homogeneous distribution of liposomes in murine melanoma that contributes to a better tumor response. Cancer Res. 2007; 67: 9455-62. 
55. van Leeuwen-van Zaane F, van Driel PB, Gamm UA, Snoeks TJ, de Bruijn HS, van der Ploegvan den Heuvel A, et al. The Effect of the Drug-Light Interval on Chlorin e 6 Photodynamic Therapy on Oral Squamous Cell Carcinomal in Mice. Fiber Optic Spectroscopy for the Optimisation of Photodynamic Therapy. Rotterdam, The Netherlands; 2013. p. 135-58.

56. Seynhaeve AL, Ten Hagen TL. High-Resolution Intravital Microscopy of Tumor Angiogenesis. In: Ribatti D, editor. Tumor Angiogenesis Assays Methods and Protocols. 1464 ed. New York: Humana; 2016. p. 115-27.

57. Deichmann R, Haase A. Quantification of T1 values by SNAPSHOT-FLASH NMR imaging. J Magn Reson. 1992; 612: 608-12.

58. Yushkevich PA, Piven J, Hazlett HC, Smith RG, Ho S, Gee JC, et al. User-guided 3D active contour segmentation of anatomical structures: Significantly improved efficiency and reliability. Neuroimage. 2006; 31: 116-1128.

59. [Internet] Comparative relaxivities and efficacies of gadolinium-based commercial contrast agents. 2013. https://cds.ismrm.org/protected/13MProceedings/files/0746.PDF.

60. Tofts PS, Kermode AG. Measurement of the blood-brain barrier permeability and leakage space using dynamic MR imaging. Magn Reson Med. 1991; 17: 357-67.

61. Kijanka M, Warnders FJ, El Khattabi M, Lub-de Hooge M, van Dam GM, Ntziachristos V, et al. Rapid optical imaging of human breast tumour xenografts using anti-HER2 VHHs site-directly conjugated to IRDye $800 \mathrm{CW}$ for image-guided surgery. Eur J Nucl Med Mol Imaging. 2013; 40: 1718-29.

62. Kijanka MM, van Brussel AS, van der Wall E, Mali WP, van Diest PJ, van Bergen En Henegouwen PM, et al. Optical imaging of pre-invasive breast cancer with a combination of VHHs targeting CAIX and HER2 increases contrast and facilitates tumour characterization. EJNMMI Res. 2016; 6: 14.

63. van Brussel AS, Adams A, Oliveira S, Dorresteijn B, El Khattabi M, Vermeulen JF, et al. Hypoxia-Targeting Fluorescent Nanobodies for Optical Molecular Imaging of Pre-Invasive Breast Cancer. Mol Imaging Biol. 2016; 18: 535-44.

64. [Internet] Photoimmunotherapy (PIT) Study in Recurrent Head/Neck Cancer for Patients Who Failed at Least Two Lines of Therapy. 2019. https://clinicaltrials.gov/ct2/show/study/

NCT03769506. 


\section{Chapter 5}

Vascular targeted photodynamic therapy: a review of the efforts towards molecular targeting of tumor vasculature

Vida Mashayekhi, Charlotte Op 't Hoog and Sabrina Oliveira

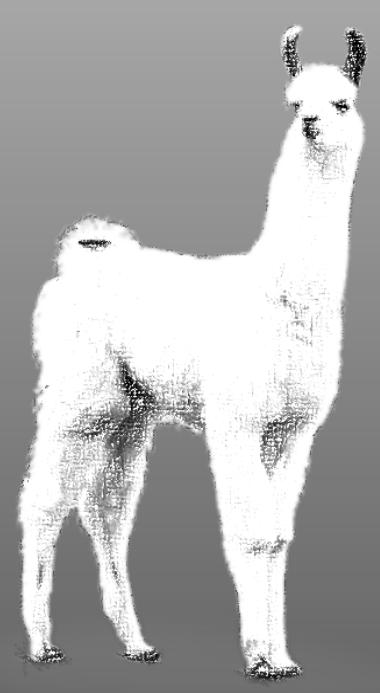




\section{ABSTRACT}

The therapeutic value of vascular targeted photodynamic therapy (VTP) for cancer has already been recognized in the clinic: $\mathrm{TOOKAD}^{\circledR}$ has been clinically approved in Europe and Israel for treatment of men with low-risk prostate cancer. When the light is applied shortly after intravenous administration of the photosensitizer, the damage is primarily done to the vasculature. This results in vessel constriction, blood flow stasis, and thrombus formation. Subsequently, the tumor is killed due to oxygen and nutrient deprivation. To further increase treatment specificity and to reduce undesired side effects, such as damaging to the surrounding healthy tissues, efforts have been made to selectively target the PS to the tumor vasculature, an approach named molecular targeted VTP (molVTP). Several receptors have already been explored for this approach, namely CD13, CD276, Extra domains of fibronectin (A, B), Integrin $\alpha v \beta 3$, Neuropilin-1, Nucleolin, PDGFR $\beta$, TF, and VEGFR-2, which are overexpressed on tumor vasculature. Preclinical studies have shown promising results, further encouraging the investigation and future application of molVTP, to improve selectivity and efficacy of cancer treatment. This strategy will hopefully lead to even more selective treatments for many cancer patients. 


\section{Introduction}

Photodynamic therapy (PDT) involves three components to create local cytotoxicity: a light-activatable drug, named photosensitizer (PS), light with a specific wavelength that can activate the PS, and oxygen. Separately, the three components are harmless. Together, the light activated PS will transfer energy to oxygen, forming cytotoxic reactive oxygen species (ROS), which can cause cytotoxicity due to the damage to biomolecules such as lipids, proteins, and nucleic acids. Consequently, the produced ROS can kill cancer cells, damage tumor vasculature, and can also induce inflammatory and immune responses.

The PDT procedure is carried out in two steps: first, the PS is administered, and this is followed by site-specific light exposure [1]. The degree in which cancer cells or tumor vasculature are preferentially killed can be influenced by varying the time between PS administration and illumination, named the drug-light interval (DLI). When a PS is administered systemically in the body, it is first present in the vasculature. Most commonly in the clinic, a 2 to 4 days DLI is employed to favour the accumulation of the PS in the tumor. This has been described to be driven by the enhanced permeability and retention (EPR) effect. This phenomenon is caused by the unorganised structure of tumor vasculature, enabling macromolecules to extravasate into the extravascular space [2]. In addition, lymphatic drainage is impaired, causing the macromolecules to be retained longer in tumor tissue. Thus, a long DLI provides a strategy to preferentially damage tumor cells using PDT, without any molecular targeted approach, which we here refer to as conventional PDT. Alternatively, using a short DLI preferentially damages tumorassociated vessels, as the PS is still present in the vasculature, which has been named vascular targeted PDT (VTP) [3]. VTP mainly damages tumor vasculature, causing vessel constriction, blood flow stasis, and thrombus formation [4]. This vascular shutdown blocks the oxygen and nutrient supply to the tumor and therefore causes necrosis of the tumor.

VTP is a promising therapeutic approach and is expected to have several advantages compared to conventional PDT. First, very hydrophobic PSs are used in conventional PDT, which are retained in the body for longer periods of time in order to accumulate in the tumor. The longer a PS is present in the body, the longer patients can experience photosensitivity after treatment [5,6]. Second, although light is applied locally in conventional PDT, surrounding healthy tissues are in many cases damaged due to poor 
tumor selectivity of the PS. Third, lack of oxygen in some areas of the tumors due to the acute hypoxia, impairs production of the cytotoxic ROS [7]. Alternatively, in the tumor vasculature, oxygen is more readily available, and the vasculature is directly accessible to the PS.

The therapeutic value of VTP for cancer has already been proven in the clinic. In November 2017, the first PS Palladium-metalated bacteriopheophorbide, known as TOOKAD $^{\circledR}$ (Negma Lerads/Steba Biotech), has been approved in Europe and Israel for the treatment of men with low-risk prostate cancer [8]. This treatment can cause fast vascular occlusion and subsequently cancer cell death, within a $5 \mathrm{~mm}$ range of the optic fibres. Currently, a phase II clinical trial is ongoing in the US, in which TOOKAD ${ }^{\circledR}$ is applied for the treatment of intermediate risk prostate cancer (NCT03315754). The water-soluble derivative of TOOKAD ${ }^{\circledR}($ WST11), is now under evaluation in phase I clinical trials for the treatment of esophagogastric cancer (NCT03133650) and upper tract urothelial carcinoma (NCT03617003).

Although VTP is already used in the clinic, efforts have been made beyond this approach to increase selectivity and efficacy of the therapy. For a more general overview of different approaches employed to increase PDT selectivity, the reader is referred to [9]. More selective accumulation of the PS at the vasculature of the tumor would improve the efficacy of the treatment and reduce the risk of side effects. Severe side effects can occur due to PS accumulation in non-targeted tissues, such as surrounding nerves and muscles, or the bladder and the rectum in particular cases of prostate cancer. In the past two decades, efforts have been made to render the PS specific to the tumor vasculature, namely by targeting particular tumor endothelial markers, a strategy here described as molecular targeted VTP or molVTP [10]. In this review, we describe research studies that have been performed in the last 15 years, focused on the molecular targeting of PSs to the tumor vasculature for more cancer specific PDT.

\section{Molecular targeting of tumor vasculature}

Certain proteins are only present or more abundant on tumor vasculature, which allows for the usage of different types of targeting moieties, such as peptides, antibodies or antibody fragments, and nanocarrier systems, to deliver the PS specifically to the tumor vasculature for molVTP [11] (Figure 1). 


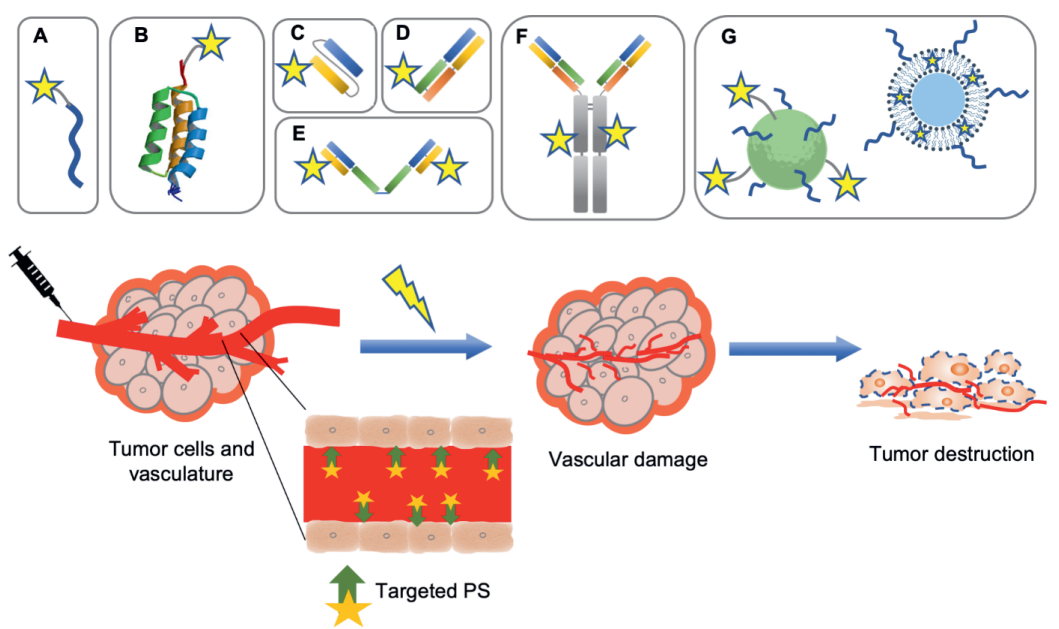

Figure 1. Schematic representation of molVTP. Different targeting moieties that have been investigated to selectively target PS to the tumor vasculature, for improving selectivity and efficacy of cancer treatment: A. small peptide, B. affibody, C. single chain variable fragment (scFv), D. antigen-binding fragment (Fab), E. small immunoprotein (SIP), F. antibody (IgG), and G. nanocarrier systems.

Importantly, some of these targets are described to be present not only on tumorassociated vasculature but also on cancer cells, thereby also leading to tumor-cell targeted PDT, or molecular targeted PDT (molPDT) [12-14]. Even though molecular targeting of tumor cells has also developed considerably over the last decade, such studies are outside the scope of this review. It is nevertheless important to highlight that when both tumor-cells and tumor-vasculature are targeted, the corresponding effects may be difficult to separate in preclinical studies. In the following sections, the proteins or markers that have been explored for molVTP are described in alphabetical order, and the overview of the chemical structures of the PSs employed is given in Figure 2. 


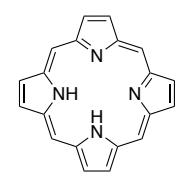

Porphyrin $[28,32,52]$

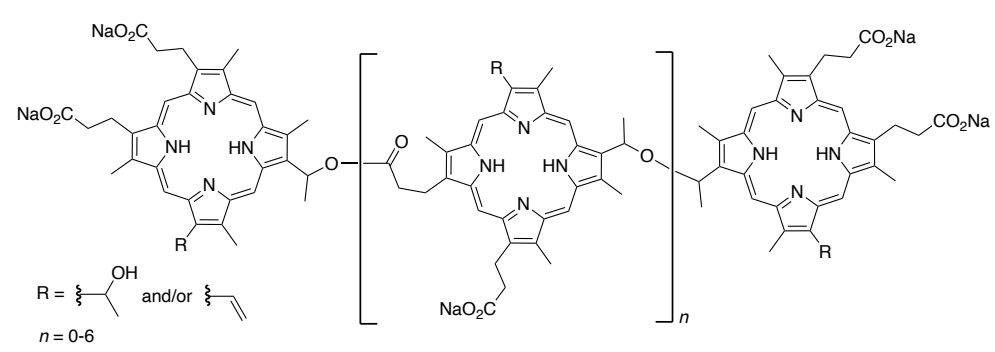

Porfimer sodium (Photofrin ${ }^{(\mathbb{B}}$ ) [57]

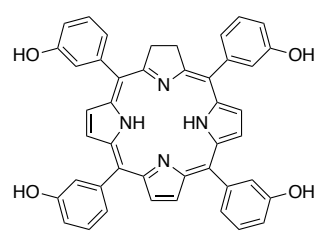

$m$-Tetrahydroxylphenyl-chlorin (mTHPC or Temoporfin) [39]

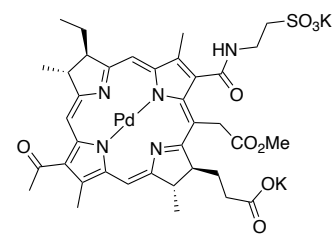

Palladium bacteriopheophorbide $\left(\right.$ TOOKAD $\left.^{\circledR}\right)[3,8]$

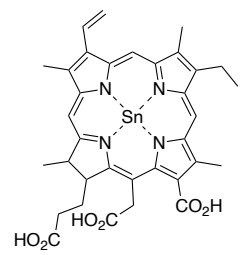

Sn(IV) chlorin e6 [31,69-71]

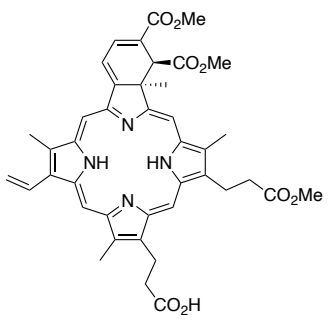

Verteporfin (Visudyne ${ }^{\circledR}$ ) [67]

Tetraphenylchlorin (TPC) [37,47-51]

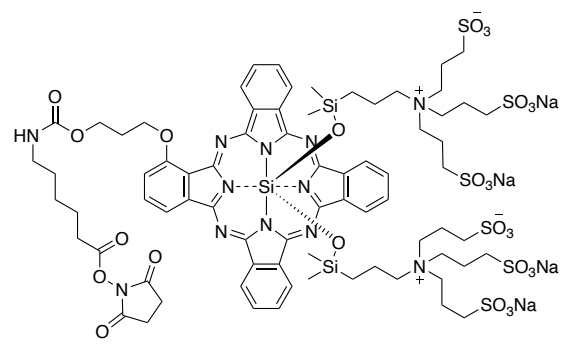

IRDye700DX [22,23,40,41,60,79]

Figure 2. Overview of the chemical structures of the PSs used in the studies discussed in this review. 


\section{CD13/aminopeptidase $\mathrm{N}$}

CD13/aminopeptidase is an ubiquitous enzyme which exists in a variety of human cell types (epithelial, endothelial, fibroblast, leukocyte) [15]. This receptor has a role in several biological processes, such as cell proliferation, secretion, invasion and angiogenesis [16]. The overexpression of this receptor in tumor cells and angiogenic vessels, makes it a potential target for molecular targeted therapies.

The peptides containing asparagine-glycine-arginine (NGR) were found to bind selectively to CD13 on tumor vasculature in vivo [17]. However, only one study described the application of the NGR peptide as targeting moiety in the context of PDT [18]. The PS diadduct malonic acid C60 (DMA-C60) was conjugated to NGR peptide and applied in vitro in human MCF-7 breast cancer cells. The cells were irradiated for $1 \mathrm{~h}$ using a light intensity of $60 \mathrm{~mW} / \mathrm{cm}^{2}$. Cell viability was significantly decreased and the highest concentration $(10 \mu \mathrm{g} / \mathrm{ml})$ caused a decrease in viability to $20 \%$. Although NGR is potentially a suitable moiety to target tumor vasculature, no studies have been conducted on the effects of DMA-C60-NGR on endothelial cells or in vivo models.

\section{CD276}

The transmembrane glycoprotein CD276 is an immunoregulatory molecule overexpressed on endothelial cells of tumor vasculature and in different types of tumors, while limited expression is seen in normal cells $[19,20]$. The physiological functions of CD276 is still unclear [21]. Due to the high level of expression in cancer and endothelial cells, CD276 could be a potential target for combined molVTP and molPDT. In a recent study conducted by Bao et al., the Fab fragment of an anti-CD276 antibody ( $\alpha \mathrm{CD} 276 / \mathrm{Fab}$ ) was conjugated to the PS IRDye700-N-hydroxysuccinimide (IR700) and the efficacy after PDT was determined in 4T1 tumor-bearing mice [22]. First, it was shown that 4T1 cells and tumor vasculature overexpress CD276, using immunofluorescence staining with an $\alpha \mathrm{CD} 276$ antibody, conjugated to Fluorescein (FITC). The FITC-staining colocalized with a marker of $41 \mathrm{~T}$ cells (integrin $\beta 6$ ) and a marker of vasculature (CD31). Thereafter, the tumor uptake of the $\alpha \mathrm{CD} 276 / \mathrm{Fab}-\mathrm{IR} 700$ was determined in vivo in mice subcutaneously xenografted with $4 \mathrm{~T} 1$ cells. A similar conjugate with irrelevant affinity was used as a control. The uptake was expressed as the percentage of fluorescence intensity, by normalizing uptake values to the total dose. The uptake of the $\alpha \mathrm{CD} 276 / \mathrm{Fab}-\mathrm{IR} 700$ and the control conjugate was $10 \%$ and $4 \%$, respectively. Thus, targeting of CD276 enhanced the 
uptake of the PS conjugate. Subsequently, the PDT efficacy was determined in 4T1 tumorbearing mice by measuring tumor growth for 16 days after the treatment. PDT was performed using a light dose of $70 \mathrm{~J} / \mathrm{cm}^{2}$ and a DLI of $2 \mathrm{~h}$. The targeted conjugate significantly inhibited tumor growth between days 8 to 16 , in contrast to the control conjugate. It was already known that $4 \mathrm{~T} 1$ cells metastasize to lungs when subcutaneously xenografted [23], and in control mice, lung metastases were evident on day 16. However, when the primary xenograft tumor was treated with the $\alpha \mathrm{CD} 276 /$ Fab-IR700, the lungs showed substantially less metastatic foci and weighed significantly less than control lungs. The $\alpha \mathrm{CD} 276 / \mathrm{Fab}-\mathrm{IR} 700$ conjugate was able to inhibit the formation of lung metastasis by recruiting the tumor infiltration of $\mathrm{CD}^{+} \mathrm{T}$ cells in treated mice. The data suggested that PDT targeted to CD276 can potentially be employed to selectively kill tumor vasculature and directly or indirectly tumor cells. The vascular effects of the conjugate were not investigated further, and more studies are needed to determine the utility of $\alpha \mathrm{CD} 276 / \mathrm{Fab}-$ IR700 in molVTP.

\section{Extra domains of fibronectin}

Among several splice variants of fibronectin, extra domain A and B (ED-A and ED-B) have been reported to be potential targets for molVTP [10]. Many angiogenic processes, such as invasion, migration, and proliferation of vascular cells are regulated by a number of cell-surface and extracellular adhesion molecules. Fibronectin as an extracellular matrix (ECM) component, expressed in a variety of normal tissues. However, ED-A and ED-B expression is undetectable or negligible in normal adult tissues [24-26].

\section{Extra domain A (ED-A)}

Using an ED-A specific antibody named F8, Rybak and co-workers found that ED-A is strongly expressed in the neovasculature of human lung and liver metastases, as well as multiple other human tumors, whereas in normal tissues the ED-A expression was negligible [26]. Furthermore, a comprehensive study of human lung tumors showed that ED-A is abundantly expressed in all important subtypes of lung cancer. In order to target this neovascular marker, a small immunoprotein (SIP) of the monoclonal F8 antibody was developed [27]. The SIP(F8) was conjugated to the PS 5-[4-(succinimide- $N$ oxycarbonyl)phenyl]-10,15,20-tris-(4- $N$-methylpyridimiumyl)porphyrin trichloride (Tri$\mathrm{PyPhSUCCMeCl}{ }^{-1}$ ) and microcirculatory effects of the conjugate mediated PDT were assessed by intravital microscopy in mice xenografted subcutaneously with human SF126 
glioma cells in a dorsal skinfold chamber [28]. molVTP was performed using a light dose of $150 \mathrm{~J} / \mathrm{cm}^{2}$ and a DLI of $12 \mathrm{~h}$. The results demonstrated that ED-A-targeted molVTP led to hypoperfused areas in the center of the tumor, as a result of stasis and thrombosis. In contrast, the hypoperfused areas were surrounded by hyperperfused areas in the tumor periphery starting $48 \mathrm{~h}$ after PDT. This could be the result of molVTP-induced hypoxia, that initiates angiogenesis [29]. When molVTP was applied only once, the hyperperfused areas led to recovery of the tumor vascular circulation, and therefore the anti-tumor effects were only temporary. Importantly, repetitive illumination $(12,24,36$, and $48 \mathrm{~h}$ after injection of the conjugate), resulted in sustained reduction of glioma growth after 5 days of observation. The positive effects after repetitive illumination might be attributed to long lasting vascular dysfunction and lower recovery capacity. Even though the authors suggested Tri-PyPhSUCCMeCl ${ }^{-1}-\mathrm{SIP}(\mathrm{F} 8)$ conjugate as a promising candidate in molVTP, in clinical context, repetitive PDT might be inconvenient for the patients.

\section{Extra domain B (ED-B)}

Interestingly, the ED-B domain is highly conserved in different species, with $100 \%$ homology between human, mouse and rat. Therefore, the same targeting moieties can be used in the clinic and in preclinical studies, which can expedite the translational step to the clinic [30].

A human IgG antibody called L19 has been developed, that binds the ED-B domain with high affinity. In this study conducted by Borsi et al., biodistribution of the different formats of L19 antibody, i.e. single-chain variable fragment (scFv) and SIP, was compared to determine which format is the most suitable for targeting applications. The results showed that the SIP might be preferable for a number of tumor targeting applications, compared to $\mathrm{scFv}$ and complete antibody ( $\mathrm{IgG})$, due to the most suitable clearance rate and in vivo stability in tumor-bearing [30].

Afterwards, the same group conjugated $\operatorname{scFv}(\mathrm{L} 19)$ and $\operatorname{SIP}(\mathrm{L} 19)$ to the PS bis(triethanolamine) $\mathrm{Sn}(\mathrm{IV})$ chlorin $\mathrm{e}_{6}\left(\mathrm{SnChe}_{6}\right)$ [31]. The molVTP efficacy of the conjugates was compared in 3 different subcutaneous tumor models: FE8 sarcoma, F9 teratocarcinoma, and C51 colon adenocarcinoma. PDT was performed using a light dose of $150 \mathrm{~J} / \mathrm{cm}^{2}$ and a DLI of $5 \mathrm{~h}$ for $\operatorname{scFv}(\mathrm{L} 19)$ and $24 \mathrm{~h}$ for SIP(L19). Seven days after treatment, the tumors were collected and weighed. In FE8 bearing mice, untreated control tumors weighed on average $1.03 \mathrm{~g}$, while the $\mathrm{SnChe}_{6}-\mathrm{ScFv}(\mathrm{L} 19)$ and $\mathrm{SnChe}_{6}-\mathrm{SIP}(\mathrm{L} 19)$ 
treated tumors weighed about $0.14 \mathrm{~g}$ and $0.04 \mathrm{~g}$, respectively. As SIP(L19)-SnChe 6 was suggested to be the most potent, further analysis was continued with this format only. SIP(L19)-SnChe ${ }_{6}$ conjugate caused an arrest in tumor growth, in all three models. In addition, tissue sections of the FE8 tumors were analysed under the microscope, which showed completely occluded vessels in the SIP(L19)-SnChe ${ }_{6}$ treated tumor. This suggested that the growth arrest happened due to the rapid blood coagulation.

In another study, SIP(L19) was conjugated to a porphyrin-based PS (5-[4(succinimide- $N$-oxycarbonyl)phenyl]-10,15,20-tris-(4-methylpyridimiumyl)-porphyrin trichlorideand) and anti-cancer effects of molVTP were assessed in 2 models grafted subcutaneously in mice, either F9 murine teratocarcinoma cells or A431 human epidermoid carcinoma cells [32]. molVTP was performed using a light dose of $60 \mathrm{~J} / \mathrm{cm}^{2}$ after $24 \mathrm{~h}$ and

$48 \mathrm{~h}$ post intravenous injection. Selective accumulation of the conjugate around the tumor vessels caused a selective disruption of the tumor neovasculature after illumination and, subsequently, inhibited long-lasting tumor growth (100 days post treatment). Furthermore, natural killer cells were shown to be essential elements for induction of long-term antitumor responses. The findings of this study supported molVTP as a promising strategy to inhibit tumor growth.

\section{Integrin av $\beta 3$}

The effects of the ECM on cell survival, proliferation and differentiation are primarily mediated by integrins, which are heterodimeric transmembrane glycoprotein receptors [33]. Multiple integrins are involved in angiogenesis, but especially $\alpha v \beta 3$ integrin has a key role in endothelial survival and migration. Furthermore, $\alpha v \beta 3$ integrin is widely expressed on tumor neovasculature, but not on the vasculature of healthy tissues, which makes it a very suitable receptor for molVTP [34].

In 1987, RGD (Arginine, Glycine and Aspartate) peptide sequence was discovered as the cell attachment site in fibronectin [35]. This sequence was found to be present in many natural ligands of $\alpha v \beta 3$ integrin receptor [36]. Without surprise, this RGD peptide was conjugated to different PSs in several studies in order to explore its potential as a tumor vasculature targeting moiety for molVTP. Importantly, $\alpha v \beta 3$ integrin receptor expression is also found in multiple types of cancer cells. Therefore, the RGD peptide is also widely studied as a potential targeting moiety for tumor-cell targeted PDT, or 
molPDT. However, in this review, only the vascular-targeting potential of RGD is discussed.

In the first paper which explored the vascular-targeting potential, linear RGD and cyclic RGDfK peptides were synthesized via solid-phase approach and then conjugated to 5-(4-Carboxyphenyl)-10,15,20-triphenylchlorin (TPC) [37]. The targeting potential of the conjugates was tested in vitro by comparing the uptake in HUVEC cells overexpressing $\alpha v \beta 3$ integrin, with the murine EMT-6 mammary carcinoma cells lacking $\alpha v \beta 3$ integrin. Results showed that both conjugates accumulated on average 5 times more in HUVEC cells than in EMT-6 cells. Subsequently, authors showed that the PDT efficacy was higher in HUVEC when using the conjugated PS, compared to the free PS, with comparable results obtained for the two forms of peptide. No phototoxicity was in fact observed in HUVEC nor in EMT-6 cells treated with the free PS.

It has been known that small cyclic peptides are more resistant to proteolysis and have the ability to bind with higher affinity to integrin receptors [38]. Another approach that has been explored to increase the avidity for $\alpha v \beta 3$ integrin binding and slow down the rapid washout of RGDfK from tumor vasculature, is by increasing the number of RGDfK peptides per PS/particle. A study by Haedicke and co-workers described the conjugation of the RGDfK peptide to silica-modified calcium phosphate nanoparticles (NPs), decorated with the PS temoporfin (mTHPC) and fluorescent molecule DY682-NHS for near-infrared fluorescence (NIRF) optical imaging [39]. The PDT potency using the NP-DY682-mTHPC was determined in mice subcutaneously xenografted with human CAL-27 tonguesquamous epithelium carcinoma cells. PDT was performed using a light dose of $100 \mathrm{~J} / \mathrm{cm}^{2}$ and a DLI of $24 \mathrm{~h}$. The reduction in tumor vascularization was assessed (up to 4 weeks after treatment) by measuring a reduction of fluorescence intensity of the contrast agent IRDye800CW RGD. Two days after PDT, apoptosis was detected in the tumor and the strongest reduction in tumor vascularization occurred 1 week after the treatment. Tumor volume was significantly reduced in three out of four mice. However, in one mouse, the outer tumor area significantly started to grow 2 weeks after the treatment. According to the authors, a possible explanation for this is the heterogenous distribution of the PS and the incomplete coverage of the tumor during illumination.

This strategy of increasing the number of RGDfK peptide per PS was also studied by Dou et al. in the context of PDT [40]. They conjugated the PS IRDye700DX and cyclic 
RGDfK (cRGD) to a polymer with a polyethylene glycol-poly L-glutamic acid (PEG-PGlu) backbone. The resulting conjugates contained 5 cRGD peptides (IR700-PEG-PGlucRGD5) or 15 cRGD peptides (IR700-PEG-PGlu-cRGD15). In addition, a control with 1 cRGD peptide (IR700-PEG-cRGD) and a control lacking affinity for $\alpha v \beta 3$ (IR700-PEGPGlu-RAD15) were used. The distribution of the conjugates was compared in vivo in mice subcutaneously xenografted with U87 cells. The IR700-PEG-PGlu-RAD15 conjugate and the free PS did not show tumor-specific accumulation. The monomeric IR700-PEG-cRGD conjugate slightly improved the accumulation. In comparison, the accumulation of IR700PEG-PGlu-cRGD5 and IR700-PEG-PGlu-cRGD15 were substantially higher, indicating that multiple peptides enhanced the avidity for binding to $\alpha v \beta 3$ integrin. Furthermore, the IR700-PEG-PGlu-cRGD5 and IR700-PEG-PGlu-cRGD15 accumulated preferentially within tumor cells and tumor neovasculature, respectively. These results showed that the distribution of the conjugates can be controlled by varying the number of cRGD peptides. Using the U87 tumor-bearing mice, the potency of the conjugates was determined in vivo. PDT was performed using a light dose of $100 \mathrm{~J} / \mathrm{cm}^{2}$ and a DLI of $3 \mathrm{~h}$. No statistical difference in tumor growth inhibition was observed between the free PS, IR700-PEGPGlu-RAD15 and the monomeric 700DX-PEG-cRGD. On the other hand, IR700-PEGPGlu-cRGD5 and IR700-PEG-PGlu-cRGD15 significantly reduced tumor growth, of which the effect of IR700-PEG-PGlu-cRGD15 was stronger, although the tumor accumulation was similar at the time of the illumination. This suggests that the preferential accumulation in tumor neovasculature improves the PDT efficacy. In conclusion, the study showed that by fine-tuning the number of cRGD peptides on this particular system, the avidity for binding $\alpha v \beta 3$ integrin can be improved and the PS distribution can be modified.

In another study conducted by Li et al., multiple cRGD peptides and PS IR700 were conjugated to human serum albumin (HSA) molecule (cRGD-PEG-HSA-IR700) [41]. Albumin is the most abundant circulating protein in blood and has already been successfully used as a drug carrier, due to the biodegradability and safety profile [42]. Furthermore, albumin has high affinity for secreted protein acidic and rich in cysteine (SPARC), which is often overexpressed in cancers' extracellular matrix [42-44]. Therefore, this characteristic can potentially improve the affinity of the nanoconjugates. The in vitro cellular uptake study showed 121-fold increase in uptake of the targeted cRGD-PEG-HSA-IR700 nanoconjugates into human TOV21G ovarian cancer cells $(\alpha v \beta 3$ 
overexpressing cells) compared to non-targeted control nanoconjugates. Moreover, cRGDPEG-HSA-IR700 selectively induced cell death in TOV21G cells with $\mathrm{EC}_{50}$ value of $\sim 10 \mathrm{nM}$, while NIH3T3 cells (no $\alpha \mathrm{v} \beta 3$ expression) were not affected. Furthermore, the results of live/dead staining confirmed induction of a strong phototoxicity in SKOV-3 spheroids treated with targeted nanoconjugates, indicating penetration of the conjugate into the 3D spheroids model.

\section{Neuropilin-1}

Neuropilin-1 (NRP-1) is known to play a role in neural development, cell survival, migration, angiogenesis, and invasion and it has been implicated in the vascularisation and progression of tumors [45].

A comprehensive analysis of NRP-1 expression in human cancer (consisting of 65 primary breast carcinomas, 95 primary colorectal adenocarcinomas, 90 primary lung carcinomas, and 59 metastases) in $98-100 \%$ of all the tumor sections, overexpression of NRP-1 was observed in the tumor-associated vessels [46]. NRP-1 overexpression was also detected in some tumor cells such as breast, prostate, and melanoma cells [12], which indicates using a NRP-1-specific moiety could lead to both tumor cell and tumor vasculature targeting. Consequently, several studies have been conducted exploring the potential of NRP-1 for cancer-cell targeted PDT (or molPDT) and molVTP.

Tirand et al., conjugated a heptapeptide (ATWLPPR) to the PS tetraphenylchlorin (TPC) via 6-aminohexanoic acid (Ahx) linker. The PDT efficacy was determined in HUVEC cells $24 \mathrm{~h}$ after incubation with free TPC or TPC-Ahx-ATWLPPR. Treatment with free TPC showed little photodynamic activity, whereas HUVECs treated with TPCATWLPPR had 10.4-fold lower viability. In nude mice, subcutaneously xenografted with human U87 glioma cells, $2.3 \%$ of the injected TPC-Ahx-ATWLPPR per gram of tumor tissue accumulated in the tumor at $1 \mathrm{~h}$, and $2.2 \%$ at $6 \mathrm{~h}$ after intravenous administration. Furthermore, immunohistochemical analysis of the tumor sections revealed that this accumulation at the tumor site is predominantly in the neovasculature. Due to the expression of NRP-1 in U87 tumor cells, this targeting strategy may potentiate tumor cell and molVTP in vivo [47]. The in vivo molVTP efficacy of TPC-Ahx-ATWLPPR was investigated later by Bechet et al., in the same U87 glioblastoma mouse model. PDT was performed using a light dose of $120 \mathrm{~J} / \mathrm{cm}^{2}$ and a DLI of $4 \mathrm{~h}$ [48]. TPC-Ahx-ATWLPPR treatment led to a significant decrease in tumor tissue blood flow compared to treatment 
with free TPC. Following the expression of tissue factor immediately post treatment, thrombi formation was observed, which resulted in blood vessels' congestion. Moreover, the induced vascular shutdown caused a significant tumor growth delay, compared to the control group. In order to improve the in vivo stability of the peptide moiety, Thomas et al. created a peptidase-resistant pseudopeptide of ATWLPPR [49]. This was performed by replacing the amide bond between amino acid $\mathrm{A}$ and $\mathrm{T}$ by $-\mathrm{CH}_{2} \mathrm{NH}-$ bond. The resulting peptide (A $\left.\psi\left[\mathrm{CH}_{2} \mathrm{NH}\right] \mathrm{TWLPPR}\right)$ was coupled to the PS TPC via Ahx linker. The results of MALDI-TOF mass spectrometry, confirmed no degradation of the conjugate, TPC-Ahx$\mathrm{A} \psi\left[\mathrm{CH}_{2} \mathrm{NH}\right] \mathrm{TWLPPR}$, up to $4 \mathrm{~h}$ after intravenous injection in mice.

Benachour et al., developed silica-based gadolinium oxide NPs, encapsulating the PS TPC and the surface-localized conventional ATWLPPR peptide. The gadolinium oxide core of the NPs was used as a MRI contrast agent. Human MDA-MB-231 breast cancer cells over-expressing the vascular neuropilin-1 (NRP-1) receptor were used to analyse in vitro PDT efficacy. The light dose that is able to kill $50 \%$ of the cells $\left(\mathrm{LD}_{50}\right)$ for NP-TPCATWLPPR-treated cells were determined for two different PS concentrations: $9.16 \mathrm{~J} / \mathrm{cm}^{2}$ $\left(0.1 \mu \mathrm{M}\right.$ PS) and $2.80 \mathrm{~J} / \mathrm{cm}^{2}(1.0 \mu \mathrm{M}$ PS). In contrast, no significant cytotoxicity was observed in the cells treated with non-targeted NPs. Therefore, the results showed that NPTPC-ATWLPPR is a selective and potent agent for therapeutic and imaging purposes [50]. Importantly, the results of the in vivo study in rats bearing an orthotopic U87 glioblastoma, demonstrated selective accumulation of targeted NPs in the endothelial cells of tumor vessels after intravenous injection.

Later, the same group developed other peptides targeting NRP-1, of which two were selected for further investigation, named DKPPR and TKPRR [51]. The advantage of these peptides is that they show higher displacement of the ligands of NRP-1, compared to ATWLPPR. The two peptides were conjugated to TPC via Ahx linker, PEG9 or PEG18 (the number refers to the number of PEG molecules) as a spacer, to ensure some distance between TPC and the peptide. The conjugation was done by solid-phase approach, to gain specific conjugation of the PS to the amino-terminal of the peptides. The stability and distribution of the conjugates were then investigated in healthy mice [51]. DKPPR conjugates showed better tissue distribution and plasma stability, therefore this peptide was chosen for the production of NRP-1-targeted AGuIX NPs (polysiloxane-based), containing the PS TPP and gadolinium as contrast agent [52]. In order to graft the targeting peptide on 
the surface of the NPs via solid-phase approach, an additional lysine (K) was added, resulting in KDKPPR as targeting moiety. To assess NPs distribution in the tumor, U87 cancer cells were implanted in a skinfold chamber in nude mice. The intravital microscopy results showed selective localisation of the targeted AGuIX-TPP-KDKPPR in tumor vasculature $1 \mathrm{~h}$ post injection and remained visible for $24 \mathrm{~h}$, in contrast to the untargeted AGuIX-TPP which was found free in the blood vessels and removed after $6 \mathrm{~h}$. In order to evaluate PDT in vitro, HUVEC cells were treated with AGuIX-TPP-KDKPPR or AGuIX-TPP for $4 \mathrm{~h}$ and then cell survival was measured after illumination (5 or $10 \mathrm{~J} / \mathrm{cm}^{2}$ ). Cells treated with targeted-NPs and illuminated with light dose of 5 and $10 \mathrm{~J} / \mathrm{cm}^{2}$, caused $50 \%$ and $98 \%$ cell death, respectively, while untargeted NPs induced low toxicity.

\section{Nucleolin/C23}

Nucleolin/C23 is mainly located in nucleus and plays a role in modulation of cellular progression. However, the expression of this protein is significantly increased in many types of cancers and also mainly localized on the cell surface of both tumor and tumor-associated endothelial cells [53]. High expression of cell surface nucleolin participates in cell adhesion, migration, and invasive behaviour [54]. A fragment of the human high-mobility group protein 2 (HMGN2), called vascular homing peptide 3 (F3), has been shown to bind to cell surface nucleolin and holds cell-penetrating properties $[55,56]$.

Reddy et al. reported the application of iron oxide NPs with the F3 peptide in rats with 9L rat glioma cells orthotopically implanted in the brain [57]. The NPs contained the PS Photofrin and iron oxide as contrast agent for imaging. PDT was performed using a DLI of $24 \mathrm{~h}$. When compared to the non-targeted NPs or free Photofrin, rats treated with the targeted NPs exhibited a significantly enhanced overall survival. The median survival of untreated mice, mice treated with the non-targeted NPs, or the targeted NPs was 7.0, 13.0 and 33 days, respectively. Out of the 5 mice treated with targeted-NPs, 2 mice were still disease free 6 months after treatment. These survival rates are promising, thus further studies are awaited to explore the efficacy of these NPs in molVTP.

The F3 peptide can potentially be explored for tumor-targeted PDT strategies as well, as NPs coated with F3 peptide have shown to give specific targeting to selected tumor cells in vitro, including 9L, MDA-MB-435, and F98 [58]. 


\section{Platelet-derived growth factor receptor $\beta$}

Thus far, only markers on endothelial cells have been discussed as potential targets for molVTP. However, it is known that the walls of tumor vasculature predominantly consist of irregularly lined endothelial cells and pericytes [59]. Consequently, pericyte-targeted PDT has also been explored, namely through targeting of the platelet-derived growth factor receptor $\beta$ (PDGFR $\beta)$ [60]. PDGFR $\beta$ is a tyrosine kinase receptor, crucial for the development of kidney, lung and cardiovascular system in the embryo. This receptor is overexpressed on the pericytes of many types of tumors, including lymphomas, colon, ovarian, prostate, lung, and breast cancers. The stimulation of PDGFR $\beta$ has been shown to increase the coverage of the tumor vessels and subsequently improve vessel function. Moreover, in some cases the activation of this receptor increased tumor growth rate [61].

To target PDGFR $\beta$, a dimeric $\mathrm{Z}_{\mathrm{PDGFR} \beta}$ affibody was developed [60]. Affibody molecules are non-immunoglobulin-derived affinity proteins based on a three-helical bundle protein domain [62]. The dimeric $\mathrm{Z}_{\mathrm{PDGFR} \beta}$ affibody was conjugated to the PS IR700 $\left(\mathrm{Z}_{\mathrm{IR} 700}\right)$ and tested in vivo in mice subcutaneously xenografted with human LS174T colorectal cancer cells. molVTP was performed using a light dose $120 \mathrm{~J} / \mathrm{cm}^{2}$ and a DLI of $4 \mathrm{~h}$. Tumor grafts were removed and weighed at different time points and HIF1 $\alpha$ expressing cells were visualized using confocal laser scanning microscopy with an antiHIF $1 \alpha$ antibody. The expression of HIF $1 \alpha$ was significantly increased in tumor tissue after treatment with $\mathrm{Z}_{\mathrm{IR} 700}$, which reflects tumor hypoxia. In addition, histological staining revealed that blood clots were present in tumor tissue $4 \mathrm{~h}$ after treatment with $\mathrm{Z}_{\mathrm{IR} 700}$ and light, in contrast to the control in the absence of light. Thus, the results showed that targeting pericytes can cause hypoxia in the tumor due to thrombosis. The average mass of mice tumor grafts after treatment with $\mathrm{Z}_{\mathrm{IR} 700}$ was approximately $20-30 \%$ of that of the mice treated with PBS or $\mathrm{Z}_{\mathrm{IR} 700}$ in the absence of light. This indicates the vascular damage can inhibit tumor growth and pericyte-targeted PDT can be an efficient approach in cancer treatment. Importantly, some cancer cells such as several types of ovarian and breast cancers also express PDGFR $\beta[63,64]$ and therefore using the $Z_{\mathrm{PDGFR} \beta}$ affibody as targeting moiety can potentially be used in the combination of molPDT and molVTP. 


\section{Tissue factor}

Yet another potential target for molVTP is Tissue Factor (TF). TF is a transmembrane glycoprotein, which plays an important role in hemostasis and thrombosis. In normal conditions, active TF is not expressed but when damage of vascular wall happens, subendothelial TF is expressed/exposed to blood circulation and binds to plasma factor VIIa [65]. In cancer, TF is expressed by active macrophages, stromal cells, and tumor-associated endothelial cells, which has been described to contribute to metastasis, tumor growth, and angiogenesis [66].

Hu et al. were the first group to describe targeting of a PS to TF using factor VII (fVII, a natural ligand of TF). In this study, murine fVII (mfVII) was conjugated to the PS verteporfin via N'-3-dimethylaminopropyl-N-ethylcarbodiimide hydrochloride (EDC) linker and the potency was determined in vitro in murine EMT6 and human MDA-MB-31 breast cancer cells. Results of in vitro PDT, using a light dose of $60 \mathrm{~J} / \mathrm{cm}^{2}$ with TF-targeted verteporfin showed three to four times more potency than the free verteporfin. In addition, the specificity for angiogenic endothelial cells was assessed using in vitro PDT, with a light dose of $36 \mathrm{~J} / \mathrm{cm}^{2}$, in HUVEC cells in presence and absence of VEGF. No statistical difference was observed in the viability of angiogenic and quiescent HUVEC treated with free verteporfin. In contrast, mfVII-verteporfin caused a significant decrease in viability in angiogenic HUVEC cells, but not in quiescent HUVEC [67]. In addition to angiogenic endothelial cells, many types of tumor cells, such as breast cancer cells, also overexpress TF [68]. Therefore, fVII-targeted PDT could be employed for the combined molPDT and molVTP, possibly having a broad therapeutic potential for cancer treatment. Later, the PDT efficacy was determined in mice subcutaneously grafted with murine breast cancer EMT6 cells. PDT treatment was performed 4-6 times with the interval of 2 or 3 days, using a light dose of $105 \mathrm{~J} / \mathrm{cm}^{2}$ and a DLI of 90 minutes. Free verteporfin did not have any effect on tumor growth, while mfVII-targeted PS was effective in inhibiting tumor growth over 18 days of observation.

Due to the high cost of extraction of verteporfin from Visudyne ${ }^{\circledR}$, the same group conjugated mfVII to $\mathrm{Sn}(\mathrm{IV})$ chlorin $\mathrm{e}_{6}\left(\mathrm{SnChe}_{6}\right)$ [69]. In vitro PDT was performed in MDA-MB-231 cells, using a light dose of $36 \mathrm{~J} / \mathrm{cm}^{2}$. Results showed that TF-targeting enhanced the ability of SnChe $_{6}$-mediated PDT to kill MDA-MB-231 cells by 12 -fold, with $\mathrm{LD}_{50}$ values (dose killing $50 \%$ of cells) of PS concentration of $0.58 \mu \mathrm{M}$ and $7.00 \mu \mathrm{M}$ for 
targeted and non-targeted $\mathrm{SnChe}_{6}$, respectively. In addition, the effect of $\mathrm{TF}$ expression level on the efficacy of targeted PDT was determined by treating human MDA-MB-231 (high TF expression), MCF-7 breast cancer cells (low expression of TF) and 293 cells (no TF expression). It was found that the phototoxicity was directly correlated to the expression level, and that 293 cells were not affected. These results suggest that TF-targeted PDT can selectively kill TF-expressing cells, without damaging surrounding cells. The mfVIISnChe $_{6}$ conjugate was also tested in vivo in mice subcutaneously grafted with murine EMT6 cells or human MDA-MB-231 cells. PDT was performed using a light dose of $72 \mathrm{~J} / \mathrm{cm}^{2}$ and a DLI of 90 minutes. EMT6 tumors treated with mfVII-SnChe 6 , weighed significantly less than that those from control mice $(\mathrm{P}<0.05)$. In MDA-MB-231 model, even though targeted PDT significantly inhibited tumor growth, the differences in tumor weight were not statistically significant $(\mathrm{P}>0.05)$.

The same group investigated the efficacy of SnChe $_{6}$-mfVII-targeted PDT in vitro and in vivo in lung cancer models [70]. Besides SnChe $_{6}$-mfVII, they also developed a mfVII-SnChe $_{6}$ conjugate, containing two repeats of the nuclear localisation sequence

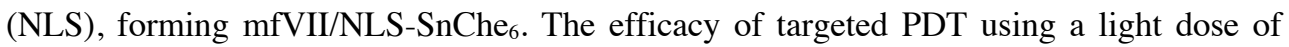
$36 \mathrm{~J} / \mathrm{cm}^{2}$ was determined in vitro, which showed that TF-targeting could enhance the killing of A549 and H460 cells up to 25-fold, in comparison to the free PS. Furthermore, mfVII/NLS-SnChe ${ }_{6}$ was slightly more effective than mfVII-SnChe 6 (no NLS). Subsequently, mfVII/NLS-SnChe ${ }_{6}$ conjugate was used for further investigation in vivo in mice subcutaneously xenografted with A549 cells. Mice were treated with PDT twice a week for six weeks, using a light dose of $120 \mathrm{~J} / \mathrm{cm}^{2}$ and a DLI of 90 minutes. Results showed that the TF-targeted $\mathrm{SnChe}_{6}$ can significantly inhibit tumors, in contrast to the free PS. Together, these studies suggest that TF-targeted $\mathrm{SnChe}_{6}$ could be a suitable treatment modality for targeting tumor neovasculature and cancer cells, namely lung and breast cancer cells

The specificity of TF for angiogenic vessels has been verified by Hu et al. [71]. Using ELISA, they tested the expression of TF on primary human vascular endothelial cells derived from three major types of human vessels: microvascular, umbilical venous, and aortic endothelial cells. TF expression was determined in presence of VEGF, as an in vitro model for angiogenic vessels and in absence of VEGF, as an in vitro model for quiescent vessels. Results showed that TF is specifically expressed on all three types of 
angiogenic vessels and not on quiescent vessels. Furthermore, fVII selectively bound angiogenic endothelial cells, but not quiescent endothelial cells, using a cell ELISA experiment. In addition, it was shown that TF-targeted PDT, using fVII-SnChe ${ }_{6}$ with a light dose of $36 \mathrm{~J} / \mathrm{cm}^{2}$, only induced apoptosis and necrosis in angiogenic endothelial cells. The non-targeted PDT using free $\mathrm{SnChe}_{6}$ had no detectable effect on both angiogenic and quiescent endothelial cells. Thus, results suggested that fVII could be used to selectively target a PS to the angiogenic vessels, such as the tumor neovasculature.

In a different study, PEG-PLGA NPs were loaded with the PS Hemoporfin (hematoporphyrin monomethyl ether) and coupled to EGFP-EGF1 [72]. EGFP-EGF1 is a fusion protein derived from fVII, which contains the specific TF binding capacity [73]. First, the NPs uptake was determined in rat brain capillary endothelial cells (BCEC), which were stimulated by tumor necrosis factor- $\alpha(\mathrm{TNF}-\alpha)$ to induce TF expression. The targeted NPs accumulated significantly more in the BCECs, than non-targeted NPs. Moreover, the TF expression after PDT was determined with western blotting and real-time PCR. Results showed that targeted NPs led to increased expression of TF in BCECs after PDT. In addition, the cellular ROS level was determined using fluorescence microscopy, which showed higher levels of intracellular ROS in BCECs treated with targeted-PDT, suggesting induction of TF expression by ROS. The EGFP-EGF1-targeted NPs were further studied in a mouse model xenografted subcutaneously with human CA46 Burkitt lymphoma cells. The tumors were harvested $24 \mathrm{~h}$ post PDT and stained for TF expression and NPs distribution. Observation of tumor sections using confocal laser scanning microscopy showed that the targeted NPs accumulated more in the tumor vasculature than in other parts of the tumor tissue and that PDT increased TF expression. Altogether, the results suggested that PDT using EGFP-EGF1-NPs containing Hemoporfin is suitable strategy for molVTP. Importantly, the strategy employed a positive feedback loop, which enhanced the targeting of the NPs: the existing TF on neovasculature was used to target the NPs to the tumor, and as a result of the ROS production after PDT, it is suggested that more TF expression is induced, enabling more accumulation of targeted NPs in the tumor vessels.

\section{Vascular endothelial growth factor receptor 2}

The vascular endothelial growth factor receptor 2 (VEGFR-2) is a $200 \mathrm{kDa}$ glycoprotein which belongs to tyrosine kinase family. This receptor plays essential role not only in physiological angiogenesis from early embryonic to adult stages, but also in 
pathological angiogenesis such as cancer [74]. This receptor is activated by its ligand VEGF, which is a mitogen for endothelial cells [75]. VEGF is upregulated in response to hypoxia which in turn upregulates the expression of VEGFR-2 in vascular endothelial cells $[76,77]$. This receptor is less abundant in normal blood vessels and is highly expressed in tumor vasculature. The significant difference in expression level makes this receptor an ideal target for selective delivery of therapeutics to tumor neovasculature [78]. However, few research groups have explored its application in molVTP in the last 15 years.

Recently, Nishimura et al. compared vascular and tumor targeted photoimmunotherapy (PIT) in a mouse model. In this study, the PS IR700 was conjugated to DC101, a monoclonal antibody targeting murine VEGFR-2, and to trastuzumab, a monoclonal antibody against human HER2. Mice xenografted subcutaneously with HER2 overexpressing human NCI-N87 gastric cancer cells and treated with either conjugates showed anti-tumor effects, although the therapeutic effect of DC101-IR700 was suggested to be the strongest and to be mediated by a decrease in tumor microvessel density [79]. Due to the upregulation of VEGFR-2 in different cancers, the authors suggested the application of this strategy for treatment of various types of cancer. Further studies are awaited exploring VEGFR2 targeting for molVTP.

\section{Conclusion}

This review provides an overview of the targeting moieties which have been explored to provide PS selectively to tumor vasculature, in order to reduce side effects and increase treatment efficacy. As described, different conjugates have been developed for molVTP, ranging from small peptides, affibodies $(\sim 7 \mathrm{kDa})$, to intermediate molecular size, as scFvs $(\sim 30 \mathrm{kDa})$, Fabs $(\sim 50 \mathrm{kDa})$, SIPs $(\sim 80 \mathrm{kDa})$, to larger IgGs $(\sim 150 \mathrm{kDa})$, and nanocarrier systems (Figure 1). In general, a larger size of the moiety causes a decrease in tumor penetration and in clearance rate, while it can increase immunogenicity. The right balance is of importance to achieve optimal PDT efficacy. However, the properties of PS affect this balance substantially, and differences in distribution and accumulation can therefore not be solely attributed to the type of targeting moiety that is used.

Different strategies have been described to increase the size or delay the clearance from the tumor vasculature. For instance, the size can be increased by adding polymers such as polyethylene glycol. Furthermore, targeting moieties can be conjugated or grafted 
on nanocarrier systems, e.g. liposomes, or on endogenous drug carriers such as albumin. Another example is the study in which 1,5 or 15 RGDfK peptides were conjugated to IR700. The conjugate containing 5 peptides accumulated preferentially in the tumor, while the conjugate containing 15 peptides accumulated preferentially in the vasculature. Thus, by modifying the conjugates, the properties can potentially be optimized.

Among the described conjugates, the vascular effects after molVTP have explicitly been shown in vivo for NRP-1-targeting verteporfin-ATWLPPR and TPCATWLPPR conjugates, ED-B-targeting SnChe $_{6}-\mathrm{SIP}(\mathrm{L} 19)$ and porphyrin-SIP(L19), ED-Atargeting TriPyPhSUCCMeCl-1-SIP(F8), $\alpha v \beta 3$ integrin-targeting calcium phosphate NPs with mTHPC and RGDfK peptides, and pericyte-targeting PDGFR $\beta$-IR700. Therefore, these conjugates can be considered in a more developed stage of preclinical molVTP research.

Concerning clinical translation, the DLI is of particular relevance. The conjugates described in this review required DLIs from 90 minutes to $24 \mathrm{~h}$. When a long DLI is required for optimal molVTP efficacy, and when this is due to a very hydrophobic PS, the risk of side effects and phototoxicity could be higher. Only two conjugates caused regression of the tumor, in contrast to tumor inhibition observed in the rest of the in vivo studies. This concerns the calcium phosphate NPs with mTHPC and RGDfK peptides and the iron oxide NPs with photofrin and the F3 peptide. Despite the positive results, both formulations required a DLI of $24 \mathrm{~h}$, which could discourage its clinical application. On the other hand, the tumor growth inhibition and vascular effects were explicitly shown with TPC-ATWLPPR and the PDGFR $\beta$-IR700 conjugates, which required a relatively short DLI of $4 \mathrm{~h}$.

In addition to the parameters described above, the expression level of the target certainly contributes to PDT efficacy. The more it is expressed at the tumor vasculature, and the less the target is expressed elsewhere in the body, the higher the molVTP efficacy will be and the fewer the chances of damaging surrounding tissues. Furthermore, some targets ( $\alpha v \beta 3$ integrin, nucleolin, NRP-1, ED-A and TF) also overexpress on certain types of tumor cells. Therefore, depending on the tumor type, one conjugate can be preferred over another due to the expression on both tumor neovasculature and tumor cells. This would enable a dual-targeting PDT strategy, which overall is expected to enhance treatment efficacy. 
Even though some targets have been more elaborately investigated compared to others, it is still too early to predict which one will first be evaluated in the clinical setting. Due to the variable experimental parameters (type of PS and targeting moiety or formulation, light dose, size of tumor, DLI, etc) in the different studies, it is hard to draw a solid conclusion regarding the most promising target and targeting moiety for molVTP. Likely, this will vary per tumor type or even perhaps per patient.

Further preclinical studies are needed to investigate the vascular effects of the conjugates and the feasibility for clinical application. Although molVTP conjugates have not been used in any clinical trials yet, the results of preclinical studies are promising, indicating potential application of molVTP to improve selectivity and efficacy of cancer treatment.

\section{Acknowledgements}

This project has received funding from the European Research Council (ERC) under the European Union's Horizon 2020 research and innovation program (grant agreement No 677582).

\section{References}

1. Fong W-P, Yeung H-Y, Lo P-C and Ng DKP. In Ho AH-P, Kim D, Somekh MG, eds. Handbook of Photonics for Biomedical Engineering. Dordrecht: Springer Netherlands, 2017; 657-681.

2. Maeda H, Nakamura H and Fang J. Adv. Drug Deliv. Rev. 2013; 65: 71-79.

3. Mazor O, Brandis A, Plaks V, Neumark E, Rosenbach-Belkin V, Salomon Y and Scherz A. Photochem. Photobiol. 2005; 81: 342-351.

4. Olivo M, Bhuvaneswari R, Lucky SS, Dendukuri N and Soo-Ping Thong P. Pharmaceuticals (Basel). 2010; 3: 1507-1529.

5. Bellnier DA, Greco WR, Nava H, Loewen GM, Oseroff AR and Dougherty TJ. Cancer Chemother. Pharmacol. 2006; 57: 40-45.

6. Nomoto $\mathrm{T}$ and Nishiyama N. In Photochemistry for Biomedical Applications. Singapore: Springer Singapore, 2018; 301-313.

7. Challapalli A, Carroll L and Aboagye EO. Clin. Transl. imaging 2017; 5: 225-253.

8. S. GI, Abdel-Rahmene A, Mark E, A. CJ, Emmanuel C, Avigdor S, T. SP and null. J. Urol. 2018; 200: 786-793.

9. Moret F and Reddi E. J. Porphyr. Phthalocyanines 2017; 21: 239-256.

10. Chen, Bin; Pongue, Brian; Hoopes, P jack; Hasan T. Crit. Rev. Eukaryot. Gene Expr. 2006; 16: 279-306.

11. Richards DA. Drug Discov. Today Technol. 2018; 30: 35-46.

12. Barr MP, Byrne AM, Duffy AM, Condron CM, Devocelle M, Harriott P, Bouchier-Hayes DJ and Harmey JH. Br. J. Cancer 2005; 92: 328-333.

13. Liu Y, Bajjuri KM, Liu C and Sinha SC. Mol. Pharm. 2012; 9: 168-175.

14. Holzer TR, Fulford AD, Nedderman DM, Umberger TS, Hozak RR, Joshi A, Melemed SA, 
Benjamin LE, Plowman GD, Schade AE, Ackermann BL, Konrad RJ and Nasir A. PLoS One 2013; 8: e80292.

15. Pasqualini R, Koivunen E, Kain R, Lahdenranta J, Sakamoto M, Stryhn A, Ashmun RA, Shapiro LH, Arap W and Ruoslahti. 2000; 60: 722-727.

16. Luan Y and Xu W. Curr. Med. Chem. 2007; 14: 639-647.

17. Pastorino F, Brignole C, Marimpietri D, Cilli M, Gambini C, Ribatti D, Longhi R, Allen TM, Corti A and Ponzoni M. Cancer Res. 2003; 63: 7400-7409.

18. Shi J, Wang Z, Wang L, Wang H, Li L, Yu X, Zhang J, Ma R and Zhang Z. Int. J. Nanomedicine 2013; 8: 1551-1562.

19. Kraan J, van den Broek P, Verhoef C, Grunhagen DJ, Taal W, Gratama JW and Sleijfer S. Br. J. Cancer 2014; 111: 149-156.

20. Picarda E, Ohaegbulam KC and Zang X. Clin. Cancer Res. 2016; 22: 3425-3431.

21. Seaman S, Zhu Z, Saha S, Zhang XM, Yang MY, Hilton MB, Morris K, Szot C, Morris H, Swing DA, Tessarollo L, Smith SW, Degrado S, Borkin D, Jain N, Scheiermann J, Feng Y, Wang Y, Li J, Welsch D, DeCrescenzo G, Chaudhary A, Zudaire E, Klarmann KD, Keller JR, Dimitrov DS and St Croix B. Cancer Cell 2017; 31: 501-515.

22. Bao R, Wang Y, Lai J, Zhu H, Zhao Y, Li S, Li N, Huang J, Yang Z, Wang F and Liu Z. Mol. Pharm. 2019; 16: 339-348.

23. Gao L, Zhang C, Gao D, Liu H, Yu X, Lai J, Wang F, Lin J and Liu Z. Theranostics 2016; 6: 627-637.

24. Risau W and Lemmon V. Dev. Biol. 1988; $125: 441-450$.

25. Zollinger AJ and Smith ML. Matrix Biol. 2017; 60-61: 27-37.

26. Rybak J-N, Roesli C, Kaspar M, Villa A and Neri D. Cancer Res. 2007; 67: 10948-10957.

27. Villa A, Trachsel E, Kaspar M, Schliemann C, Sommavilla R, Rybak JN, Rösli C, Borsi L and Neri D. Int. J. Cancer 2008; 122: 2405-2413.

28. Acker G, Palumbo A, Neri D, Vajkoczy P and Czabanka M. J. Neurooncol. 2016; 129: 33-38.

29. Gallagher-Colombo SM, Maas AL, Yuan M and Busch TM. Isr. J. Chem. 2012; 52: 1-10.

30. Borsi L, Balza E, Bestagno M, Castellani P, Carnemolla B, Biro A, Leprini A, Sepulveda J, Burrone O, Neri D and Zardi L. Int. J. Cancer 2002; 102: 75-85.

31. Fabbrini M, Trachsel E, Soldani P, Bindi S, Alessi P, Bracci L, Kosmehl H, Zardi L, Neri D and Neri P. Int. J. Cancer 2006; 118: 1805-1813.

32. Palumbo A, Hauler F, Dziunycz P, Schwager K, Soltermann A, Pretto F, Alonso C, Hofbauer GF, Boyle RW and Neri D. Br. J. Cancer 2011; 104: 1106-1115.

33. Avraamides CJ, Garmy-Susini B and Varner JA. Nat. Rev. Cancer 2008; 8: 604-617.

34. Desgrosellier JS and Cheresh DA. Nat. Rev. Cancer 2010; 10: 9-22.

35. Ruoslahti E and Pierschbacher M. Science. 1987; 238: 491-497.

36. Sheldrake H and Patterson L. Curr. Cancer Drug Targets 2009; 9: 519-540.

37. Frochot C, Stasio B Di, Vanderesse R, Belgy MJ, Dodeller M, Guillemin F, Viriot ML and Barberi-Heyob M. Bioorg. Chem. 2007; 35: 205-220.

38. Verrier S, Pallu S, Bareille R, Jonczyk A, Meyer J, Dard M and Amédée J. Biomaterials 2002; 23: 585-596.

39. Haedicke K, Kozlova D, Gräfe S, Teichgräber U, Epple M and Hilger I. Acta Biomater. 2015; 14: 197-207.

40. Dou X, Nomoto T, Takemoto H, Matsui M, Tomoda K and Nishiyama N. Sci. Rep. 2018; 8: 1 12.

41. Li F, Zhao Y, Mao C, Kong Y and Ming X. Mol. Pharm. 2017; 14: 2793-2804.

42. Liu $Z$ and Chen X. Chem. Soc. Rev 2016; 45: 1432-1456.

43. Desai N, Trieu V, Damascelli B and Soon-Shiong P. Transl. Oncol. 2009; 2: 59-64.

44. Shi Q, Bao S, Song L, Wu Q, Bigner DD, Hjelmeland AB and Rich JN. Oncogene 2007; 26: 4084-4094.

45. Guo H-F and Vander Kooi CW. J. Biol. Chem. 2015; 290: 29120-29126.

46. Jubb AM, Strickland LA, Liu SD, Mak J, Schmidt M and Koeppen H. J. Pathol. 2012; 226: 5060.

47. Tirand L, Frochot C, Vanderesse R, Thomas N, Trinquet E, Pinel S, Viriot ML, Guillemin F and 
Barberi-Heyob M. J. Control. Release 2006; 111: 153-164.

48. Bechet D, Tirand L, Faivre B, Plénat F, Bonnet C, Bastogne T, Frochot C, Guillemin F and Barberi-Heyob M. Pharm. Res. 2010; 27: 468-479.

49. Thomas N, Pernot M, Vanderesse R, Becuwe P, Kamarulzaman E, Da Silva D, François A, Frochot C, Guillemin F and Barberi-Heyob M. Biochem. Pharmacol. 2010; 80: 226-235.

50. Benachour H, Sève A, Bastogne T, Frochot C, Vanderesse R, Jasniewski JD, Miladi I, Billotey C, Tillement O, Lux F and Barberi-Heyob M. Theranostics 2012; 2: 889-904.

51. Kamarulzaman E, Gazzali A, Acherar S, Frochot C, Barberi-Heyob M, Boura C, Chaimbault P, Sibille E, Wahab H and Vanderesse R. Int. J. Mol. Sci. 2015; 16: 24059-24080.

52. Thomas E, Colombeau L, Gries M, Peterlini T, Mathieu C, Thomas N, Boura C, Frochot C, Vanderesse R, Lux F, Barberi-Heyob M and Tillement O. Int. J. Nanomedicine 2017; 12: 70757088 .

53. Jia W, Yao Z, Zhao J, Guan Q and Gao L. Life Sci. 2017; 186: 1-10.

54. Qiu W, Wang G, Sun X, Ye J, Wei F, Shi X and Lv G. Med. Oncol. 2015; 32: 1-8.

55. Porkka K, Laakkonen P, Hoffman JA, Bernasconi M and Ruoslahti E. Proc. Natl. Acad. Sci. 2002; 99: 7444-7449.

56. Ruoslahti E, Duza T and Zhang L. Curr. Pharm. Des. 2005; 11: 3655-3660.

57. Reddy GR, Bhojani MS, Mcconville P, Moody J, Moffat BA, Hall DE, Kim G, Koo Y-EL, Woolliscroft MJ, Sugai J V, Johnson TD, Philbert MA, Kopelman R, Rehemtulla A and Ross BD. Clin. Cancer Res. 2006; 12: 6677-6686.

58. Hah HJ, Kim G, Lee Y-EK, Orringer DA, Sagher O, Philbert MA and Kopelman R. Macromol. Biosci. 2011; 11: 90-99.

59. Chang WG, Andrejecsk JW, Kluger MS, Saltzman WM and Pober JS. Cardiovasc. Res. 2013; 100: 492-500.

60. Shi Q, Tao Z, Yang H, Fan Q, Wei D, Wan L and Lu X. Drug Deliv. 2017; 24: 1818-1830.

61. Paulsson J, Sjöblom T, Micke P, Pontén F, Landberg G, Heldin C-H, Bergh J, Brennan DJ, Jirström K and Östman A. Am. J. Pathol. 2009; 175: 334-341.

62. Feldwisch J, Tolmachev V, Lendel C, Herne N, Sjöberg A, Larsson B, Rosik D, Lindqvist E, Fant G, Höidén-Guthenberg I, Galli J, Jonasson P and Abrahmsén L. J. Mol. Biol. 2010; 398: 232-247.

63. Wilczynski SP, Chen YY, Chen W, Howell SB, Shively JE and Alberts DS. Hum. Pathol. 2005; 36: 242-249.

64. Cristofanilli M, Morandi P, Krishnamurthy S, Reuben JM, Lee BN, Francis D, Booser DJ, Green MC, Arun BK, Pusztai L, Lopez A, Islam R, Valero V and Hortobagyi GN. Ann. Oncol. 2008; 19: 1713-1719.

65. Butenas S. Scientifica (Cairo). 2012; 2012: 1-15.

66. Han X, Guo B, Li Y and Zhu B. J. Hematol. Oncol. 2014; 7: 1-8.

67. Hu Z, Rao B, Chen S and Duanmu J. BMC Cancer 2010; 10: 1-13.

68. Contrino J, Hair G, Kreutzer DL and Rickles FR. Nat. Med. 1996; 2: 209-215.

69. Hu Z, Rao B, Chen S and Duanmu J. Breast Cancer Res. Treat. 2011; 126: 589-600.

70. Cheng J, Xu J, Duanmu J, Zhou H, J. Booth C and Hu Z. Curr. Cancer Drug Targets 2011; 11: 1069-1081.

71. Hu Z, Cheng J, Xu J, Ruf W and Lockwood CJ. Angiogenesis 2017; 20: 85-96.

72. Shi W, Yin Y, Wang Y, Zhang B, Tan P, Jiang T, Mei H, Deng J, Wang H, Guo T, Pang Z and Hu Y. Oncotarget 2017; 8: 32212-32227.

73. Mei H, Hu Y, Wang H, Shi W, Deng J and Guo T. J Huazhong Univ Sci Technol [Med Sci] 2010; 30: 42-47.

74. Shibuya M. J. Biochem. 2013; 153: 13-19.

75. Ferrara N. Nat. Rev. Cancer 2002; $2: 795-803$.

76. Ferrara N. Endocr. Rev. 2004; 25: 581-611.

77. Liu Z, Qi L, Li Y, Zhao X and Sun B. BMC Cancer 2017; 17: 1-11.

78. Behdani M, Zeinali S, Karimipour M, et al., and Muyldermans S. N. Biotechnol. 2013; 30: 205209.

79. Nishimura T, Mitsunaga M, Ito K, Kobayashi H and Saruta M. Gastric Cancer 2019: 1-13. 


\section{Chapter 6}

Dual targeting of endothelial and cancer cells potentiates in vitro nanobody-targeted photodynamic therapy

Vida Mashayekhi, Katerina T. Xenaki, Paul van Bergen en Henegouwen, Sabrina Oliveira

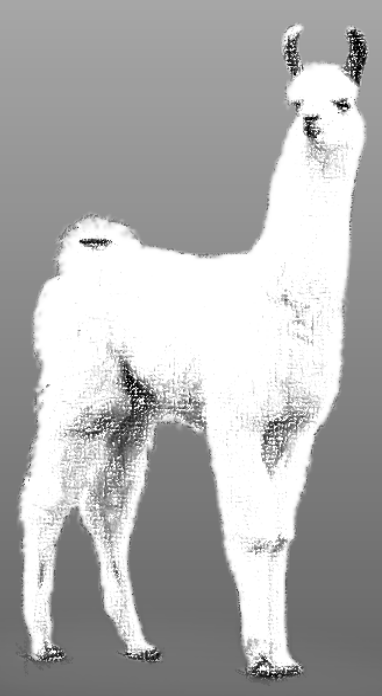




\section{ABSTRACT}

Photodynamic therapy (PDT) induces cell death through local light activation of a photosensitizer, though sub-optimal tumor specificity and side effects have hindered its clinical application. We have introduced a new strategy named nanobody-targeted PDT in which photosensitizers are delivered to tumor cells by means of nanobodies. As efficacy of targeted PDT can be hampered by heterogeneity of target expression and/or moderate/low target expression levels, we explored the possibility of combined targeting of endothelial and cancer cells in vitro. We developed nanobodies binding to the mouse VEGFR2, which is overexpressed on tumor vasculature, and combined these with nanobodies specific for the cancer cell target EGFR. The nanobodies were conjugated to the photosensitizer IRDye700DX and specificity of the newly developed nanobodies was verified using several endothelial cell lines. The cytotoxicity of these conjugates was assessed in monocultures and in co-cultures with cancer cells, after illumination with an appropriate laser. Results showed that the anti-VEGFR2 conjugates are specific and potent PDT agents. Nanobody-targeted PDT on co-culture of endothelial and cancer cells showed improved efficacy, when VEGFR2 and EGFR targeting nanobodies were applied simultaneously. Altogether, dual targeting of endothelial and cancer cells is a promising novel therapeutic strategy for more effective nanobody-targeted PDT. 


\section{Introduction}

Photodynamic therapy (PDT) is a minimally invasive approach for cancer treatment. In this approach three essential elements are needed to induce local cytotoxicity: a light-activatable photosensitizer (PS), light of a specific wavelength, and oxygen molecules. The activated PS can transfer energy to oxygen and subsequently form cytotoxic reactive oxygen species (ROS), among other reactive molecular species [1]. The produced reactive species destroy tumor cells, damage tumor vasculature, and also induce immune response [2]. Currently, PDT is used in the clinic for the treatment of cancers such as bladder, skin, head and neck and prostate [3-5]. Due to the hydrophobicity of most of the clinically approved PSs, site-specific light should be applied 2-4 days after the systemic administration of the PS in order to favor the retention of the PS in the tumor. This is mainly driven by the enhanced permeability and retention (EPR) effect [6] due to the unorganised structure of tumor vasculature, enabling therapeutics (mainly (lipo)protein associated PS) to extravasate into the extravascular space [7]. In addition, impairedlymphatic drainage causes the macromolecules to be retained longer in the tumor [6]. Therefore, longer drug to light intervals are employed in order to preferably damage tumor tissue and, more importantly, reduce damage to normal tissues. Alternatively, applying light shortly after the administration of the PS (0-30 $\mathrm{min})$ preferentially damages tumorassociated vessels. This therapeutic strategy has been named vascular targeted PDT (VTP) [8]. In this approach, which is driven passively, illumination of the PS mainly confined to the blood vessels causes vessel constriction, blood flow stasis, and thrombus formation. The vascular shutdown after VTP, can block nutrients and oxygen supply to the tumor, resulting in necrosis and tumor regression [9]. The therapeutic benefit of VTP has been recently recognized in the clinic. The PS known as TOOKAD $^{\circledR}($ Negma Lerads/Steba Biotech), has been approved in 2017 in Europe and Israel for the treatment of men with low-risk prostate cancer [10].

Although VTP and conventional PDT are already used in the clinic, in the last decades efforts have been made to increase specificity and efficacy of the therapy. Next to the local and temporal control of light application, accumulation of the PS specifically and selectivity at the tumor tissue and tumor associated vasculature, can improve the efficacy of the treatment and further decrease side effects, such as photosensitivity and damage to the surrounding nerves and muscles. To this end, certain proteins which only express or are 
more abundant on tumor cells/vasculature have been targeted using different targeting moieties, such as peptides, antibodies or antibody fragments, and nanocarrier systems, to deliver the PS specifically and selectively to the tumor tissue/vasculature [11]. Nanobodytargeted PDT is one such approach, which was developed in our group. In this approach, PS molecules are specifically associated with tumor cells by means of nanobodies. Nanobodies (NBs) are the variable domain of heavy chain only antibodies that are naturally found in camelids and considered as the smallest antigen binding fragments [12]. Nanobodies are ten-times smaller than conventional antibodies $(15 \mathrm{kDa}$, compared to $150 \mathrm{kDa}$ ), which allows them to penetrate the tumor effectively and clear faster from the body when not associated with their target $[13,14]$. Moreover, low immunogenicity potential and high solubility make them an ideal targeting moiety for targeted therapies [15]. In our previous studies, EGFR [16], c-Met [17] and US28 [18] targeted nanobodies conjugated to the photosensitizer IRDye700DX showed specific and potent cytotoxic effects on cells overexpressing these targets. As a proof of principle study, nanobodytargeted PDT was applied on an oral squamous cell carcinoma orthotopic mouse tumor model overexpressing EGFR. Light was applied $1 \mathrm{~h}$ post injection of the EGFR targeted nanobody-PS conjugates, leading to approximately $90 \%$ of tumor necrosis and importantly minimal damage to the surrounding normal tissues [19]. In a more recent study, HER2 targeted nanobody-PS conjugates were injected intravenously in HER2-positive breast cancer orthotopic mouse tumor model. Illumination $2 \mathrm{~h}$ later induced significant tumor regression after a single nanobody-targeted PDT treatment [20].

Following up on the promising results we obtained in both in vitro and in vivo studies, we explored the possibility of combined targeting of endothelial and cancer cells in vitro, in order to improve the efficacy of targeted PDT. We hypothesized that dual targeting of receptors on endothelial and cancer cells is likely beneficial in tumors with high heterogeneity of target expression and/or intermediate/low target expression levels [21,22]. In line with this, our previous study showed that dual targeting of two cancer cell targets, namely HER 2 and CAIX, improved tumor imaging in an orthotopic model of breast cancer [23]. For VTP, one of the target proteins which is mainly overexpressed on tumorassociated vessels, is vascular endothelial growth factor receptor 2 (VEGFR2). This receptor belongs to the tyrosine kinase family and has been shown to play an essential role in tumor angiogenesis and progression [24]. VEGFR2 is activated upon binding of its 
natural ligand (VEGF). This initiates a phosphorylation cascade that ultimately results in the proliferation and migration of endothelial cells [25]. Under hypoxic conditions, VEGF is upregulated leading to the subsequent upregulation of VEGFR2 expression in vascular endothelial cells, ultimately leading to neovascularization [26,27]. Higher expression of VEGFR2 on tumor-associated vessels and lower expression in normal blood vessels, makes this receptor an ideal target for selective delivery of therapeutics to the tumor neovasculature [28]

To enable targeting of endothelial cells with nanobody-targeted PDT, we have developed and characterized novel nanobodies targeting mouse VEGFR2. Nanobodies were conjugated to IRDye700DX and the phototoxicity induced by the conjugates was assessed in vitro on murine endothelial cell lines with different levels of VEGFR2 expression in monocultures. For simultaneous targeting of endothelial and cancer cells, a previously described EGFR targeted nanobody-PS conjugate was employed [16,19] and in co-cultures an oral squamous cell carcinoma cell line was combined with a murine endothelial cell line. Our results confirm the hypothesis that dual targeting of endothelial and cancer cells leads to more potent PDT.

\section{Experimental section}

\section{Cell lines}

The following murine cell lines were used in this study: brain endothelial cells (bEnd.3), pancreatic islet endothelial cells (MS1), heart endothelial cells (H5V), and fibroblast cells (NIH 3T3). bEnd.3 and H5V cell lines were generous gifts from prof. Enrico Mastrobattista (Department of Pharmaceutical Sciences, Utrecht) and prof. Ingrid Molema (Department of Pharmacology, Groningen) respectively. MS1 (ATCC ${ }^{\circledR}$ CRL2279TM) and NIH 3 T3 were (ATCC ${ }^{\circledR}$ CRL-1658 ${ }^{\mathrm{TM}}$ ) purchased from ATCC. All cell lines except MS1 were cultured in Dulbecco's modified eagle's medium (Lonza) containing $4.5 \mathrm{~g} / \mathrm{l}$ glucose and glutamine supplemented with $1 \%$ penicillin/streptomycin (SigmaAldrich) and $10 \%$ fetal bovine serum (FBS, Sigma-Aldrich). MS1 cells were grown in DMEM from ATCC (30-2002 $\left.2^{\mathrm{TM}}\right)$. Oral squamous cell carcinoma cell line OSC-19-luc2cGFP (OSC, Radiology and Molecular Imaging department, Leiden), were cultured as described previously [19]. 


\section{Llama immunization and Library construction}

Immunizations of two llamas were performed at Kaneka Eurogentec S.A (Liege, Belgium). Animals received four injections of murine VEGFR2/Fc purified protein ( $25 \mu \mathrm{g}$ per injection; R\&D systems) with interval of 2 weeks. As a boost injection, membrane preparations derived from $10^{8}$ bEnd. 3 cells were injected 2 weeks after the final injection [29]. Specific immune response was confirmed by testing pre-immune serum and serum after the second and the final boost. The total mRNA isolated from PBLs was transcribed to cDNA and specific primers were used to amplify the $\mathrm{VhH}$ regions which were eventually ligated into the phagemid vector pUR8100-Myc-His as described previously [30]. Transformation of electrocompetent TG1 cells resulted in the generation of two libraries of approximately $0.7 \times 10^{7}$ transformants each, which were further used for phage display.

\section{Phase display selections and phage ELISA}

Two rounds of phage selections on purified protein were performed in order to identify the specific VEGFR2 binding nanobodies. Phage selection was performed as described previously [30] with the only difference being the amount of coated protein which was $1 \mu \mathrm{g}$ of VEGFR2/Fc protein or equimolar of an irrelevant protein with Fc tail. For phage ELISA, the protocol described previously was followed [30] except that the amount of coated protein was $300 \mathrm{ng} /$ well VEGFR2/Fc or equimolar of an irrelevant protein with Fe tail.

\section{Nanobody production and purification}

The nanobody sequences were re-cloned in a modified pET21 to introduce a $\mathrm{N}$-terminal pelB signal sequence and a C-terminal EPEA tag. The resulting plasmids were transformed in heat-shock competent E. coli BL21. Single colonies were grown overnight in YT-2x medium supplemented with $100 \mu \mathrm{g} / \mathrm{ml}$ ampicillin and $2 \%$ glucose. Nanobodies were produced in a 51 bioreactor (Eppendorf BioFlo 115 benchtop fermentor) in Terrific Broth (TB) medium containing $0.1 \%$ glucose and $100 \mu \mathrm{g} / \mathrm{ml}$ ampicillin and overnight preculture $(1 \mathrm{ml} / 100 \mathrm{ml} \mathrm{TB})$. Once bacterial growth was in the log phase, isopropyl $\beta$-D-1-thiogalactopyranoside (IPTG) with final concentration of $1 \mathrm{mM}$ was added to induce nanobody expression overnight at $25^{\circ} \mathrm{C}$. The cells were then centrifuged at $4800 \mathrm{~g}$ for $20 \mathrm{~min}$ at $4{ }^{\circ} \mathrm{C}$ and the resulting pellet was resuspended in $300 \mathrm{ml}$ PBS. After 2 freeze-thaw cycles, periplasmic fraction was separated by centrifugation at $10000 \mathrm{~g}$ for $20 \mathrm{~min}$ at $4{ }^{\circ} \mathrm{C}$. 
The nanobodies were purified by Äkta Xpress chromatography system (GE healthcare) using $5 \mathrm{ml} \mathrm{C-tag} \mathrm{column} \mathrm{(ThermoFisher} \mathrm{Scientific)} \mathrm{and} 5 \mathrm{ml}$ HiTrap Desalting column (GE healthcare). The purity of the nanobodies was verified by SDS-PAGE under reducing condition.

\section{Conjugation of the nanobodies to photosensitizer IRDye700DX NHS and Alexa Fluor ${ }^{\mathrm{TM}} 647$ NHS Ester}

The nanobodies were conjugated to PS/fluorophore via random NHS-mediated coupling to lysine amino acids, adapted from [16]. Briefly, nanobodies (2 mg/ml) were incubated with 4 molar equivalents of the PS (LI-COR) for $3 \mathrm{~h}$ at room temperature (RT). The NB-PS conjugates were purified using four PD-10 desalting columns pre-equilibrated with PBS, as recommended by the manufacturer (GE healthcare). To conjugate nanobodies to Alexa647 NHS Ester (ThermoFisher Scientific), $2 \mathrm{mg} / \mathrm{ml}$ nanobodies were incubated with 3 molar equivalents of fluorophore for $2 \mathrm{~h}$ at RT. The conjugates were purified from free fluorophore using three Zeba spin desalting columns ( $2 \mathrm{ml}$, ThermoFisher Scientific) which were pre-equilibrated with PBS. The purity of the conjugates was determined by SDS-PAGE. Immediately after running the gel, the fluorescence of PS/Alexa647 was detected with an Odyssey infrared scanner at $700 \mathrm{~nm}$. Total protein was subsequently visualized with PageBlue ${ }^{\mathrm{TM}}$ staining. The degree of conjugation (DOC) was determined following the manufacturer's protocol by measuring the absorbance at 280 and $689 \mathrm{~nm}$ for PS or 280 and $650 \mathrm{~nm}$ for Alexa647 using a Nanodrop spectrophotometer (Nanodrop Technologies, Wilmington, Delaware, USA). For the co-culture experiment, 7D12-PS conjugate with DOC of 0.5 was prepared as described previously [19].

\section{Determination of binding affinity}

Mouse VEGFR2 (His tag, Sino Biological, 300 ng/well), equimolar of human VEGFR2 (His tag, Sino Biological) and mouse VEGFR1 (R\&D systems) proteins were coated on MaxiSorp ${ }^{\mathrm{TM}}$ plate (ThermoFisher Scientific) overnight at $4{ }^{\circ} \mathrm{C}$. Next day, wells were washed twice with PBS and blocked with $4 \%$ milk in PBS (mPBS) for 30 min at RT. A dilution range of the nanobodies, NBs-PS and NB-Alexa647 conjugates $(0.1 \mathrm{nM}-$ $100 \mathrm{nM}$ ) in $2 \%$ mPBS was added to the wells in triplicate and incubated for $2 \mathrm{~h}$ at RT. For the labeled nanobodies, after 3 times washing with PBS, fluorescence was detected with an Odyssey infrared scanner at $700 \mathrm{~nm}$. For the unlabeled nanobodies, wells were washed 3 times with $2 \% \mathrm{mPBS}$, followed by the addition of rabbit anti-VHH antibody (QVQ 
B.V.). After $1 \mathrm{~h}$ incubation at RT, wells were washed with $2 \% \mathrm{mPBS}$ and then incubated with IRDye800CW goat anti rabbit secondary antibody (LI-COR). The plates were scanned the same way as mentioned above. Data were analyzed with GraphPad Prism 8.0 software. The experiments were performed at least three times and data shown as mean \pm SD.

\section{Flow cytometry analysis}

First, four murine cell lines were seeded in 48 well plates (25000 cells/well, Nunc, Roskilde, Denmark). Next day, cells were incubated with monoclonal anti-VEGFR2-PE

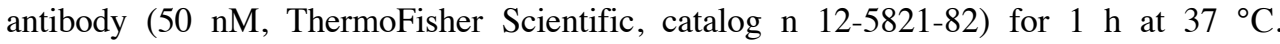
Afterwards, cells washed three times with PBS and then trypsinized. The cells were washed once with PBS and then resuspended in 1\% BSA-PBS. The same protocol was used for the NB-Alexa647 conjugates $(50 \mathrm{nM})$. The unstained controls for each cell line were taken along. Measurement was carried out on the BD FACSCanto II (BD Biosciences) with standard filter sets. Analysis was performed using FlowLogic ${ }^{\mathrm{TM}}$ software version 7.3 (Inivai Technologies).

\section{In vitro association without and with pre-blocking}

In order to determine association of the NB-PS conjugates with cells, four murine cell lines were seeded (8000 cells/well) in 96 well plates one day before the experiment. Cells were incubated with VEGFR2 targeted NB-PS conjugates $(0.78 \mathrm{nM}-200 \mathrm{nM})$ in DMEM medium without phenol red supplemented with $10 \%$ FBS, $1 \%$ penicillin/streptomycin for $1 \mathrm{~h}$ at $37{ }^{\circ} \mathrm{C}$. After 2 times washing with DMEM medium, the total fluorescence of the associated conjugates was measured using an Odyssey infrared scanner at $700 \mathrm{~nm}$.

To explore association of the NB-PS conjugates with cells in the presence of antiVEGFR2 antibody or unconjugated nanobodies, bEnd.3 cells were pre-incubated with $250 \mathrm{nM}$ anti-VEGFR2 antibody (ThermoFisher Scientific) or unconjugated nanobodies for $10 \mathrm{~min}$ at RT, followed by the addition of the NB-PS conjugates $(25 \mathrm{nM})$ and incubation for $1 \mathrm{~h}$ at $37^{\circ} \mathrm{C}$. NB-PS conjugates were added alone to the cells in order to obtain $100 \%$ fluorescent intensity. Cells were washed twice with PBS and total fluorescence was detected at $700 \mathrm{~nm}$. Data were analyzed with GraphPad Prism 8.0 software and shown as mean \pm SD. Analysis of significance was performed for each nanobody through unpaired $\mathrm{t}$ test. $\mathrm{p}<0.05$ was considered significant. 


\section{Immunofluorescence microscopy}

Microscopic evaluation of the NB-Alexa647 accumulation in murine cell lines under static conditions

Ten thousand cells were seeded a day before the experiment in 16 well Lab-Tek chamber slides (ThermoFisher Scientific). Fifty nanomolar concentration of the NBAlexa647 conjugates was incubated with the cells for $1 \mathrm{~h}$ at $37^{\circ} \mathrm{C}$. Then, cells were washed three times with PBS and fixed with $4 \%$ solution of formaldehyde in PBS for 30 min at RT. Formalin-induced autofluorescence was quenched after incubation with $100 \mathrm{mM}$ glycine in PBS for $10 \mathrm{~min}$ at RT. Cells were permeabilized with $0.1 \%$ Triton X-100 in PBS for $5 \mathrm{~min}$ at RT followed by washing with PBS. As control, anti-VEGFR2 monoclonal antibody (55B11, Cell Signaling) was used, following the protocol recommended by the manufacturer. After $1 \mathrm{~h}$ incubation with the antibody, wells were washed three times with 0.1\% BSA-PBS, and subsequently, Alexa Fluor ${ }^{\mathrm{TM}} 488$ Phalloidin (final concentration 33 $\mathrm{nM}$, ThermoFisher Scientific) in $0.1 \%$ BSA-PBS was added to the wells and incubated for 30 min. After two times washing with PBS, cells were incubated with 4',6-diamidino-2phenylindole (DAPI, $0.25 \mu \mathrm{g} / \mathrm{ml}$, ThermoFisher Scientific) for $5 \mathrm{~min}$. The slides were mounted with SlowFade (Invitrogen) and imaged with LSM700 confocal laser scanning microscope using 40x oil immersion objective (Carl Zeiss Microscopy, Jena, Germany). The images were analyzed with ImageJ.

Microscopic evaluation of the NB-Alexa647 accumulation in bEnd. 3 cells under flow conditions

The experiments under flow were performed using ibidi pump system ${ }^{\circledR}$. bEnd.3 cells were seeded in a $0.6 \mathrm{~mm} \mu$-Slide I Luer in DMEM medium containing $10 \%$ FBS and $1 \%$ penicillin/streptomycin at a density of $1.6 \times 10^{6}$ cells per slide. The slides were incubated for $2 \mathrm{~h}$ at $37^{\circ} \mathrm{C}$ and then connected to a pump-controlled perfusion set under unidirectional and continuous flow (shear rate $300 / \mathrm{sec}$, shear stress $0.3 \mathrm{~N} / \mathrm{m}^{2}$, viscosity $1 \mathrm{mPa} . \mathrm{s})$ at $37^{\circ} \mathrm{C}, 5 \% \mathrm{CO}_{2}$ for 5 days before addition of the conjugates. The medium was changed every 2 days. The conjugates diluted in complete medium were added to the system (final concentration $50 \mathrm{nM}$ ) while flow was shortly stopped. Cells were incubated with the conjugates for $1 \mathrm{~h}$ at $37^{\circ} \mathrm{C}$ under flow (shear rate $300 / \mathrm{sec}$, shear stress $0.3 \mathrm{~N} / \mathrm{m}^{2}$, viscosity $1 \mathrm{mPa} . \mathrm{s})$. Afterwards, the flow stopped, the slide was disconnected from the system and cells were carefully washed three times with PBS. The rest of the cell 
preparation for confocal microscopy was performed as mentioned above expect for the last step, in which ibidi mounting medium was used before imaging.

\section{Western blot analysis of VEGFR2 Phosphorylation}

MS1 cells (250000 cells/well) were seeded in 6 well plates in DMEM medium containing $10 \%$ FBS and $1 \%$ penicillin/streptomycin and allowed to adhere overnight. Next day, the cells were rinsed with DMEM containing 0.1\% FBS and serum-starved overnight in the same medium. The day of the assay, $50 \mathrm{nM}$ VEGF-A (PEPROTECH ${ }^{\circledR}$ ) or $1 \mu \mathrm{M}$ nanobodies were added to the cells in the starvation medium and incubated for $15 \mathrm{~min}$ at $37^{\circ} \mathrm{C}$. Afterwards, cells were immediately washed twice with ice-cold PBS and total cell lysates were prepared with scraping cells in $100 \mu \mathrm{l}$ of 1x Laemmli sample buffer and boiled for $10 \mathrm{~min}$ at $100{ }^{\circ} \mathrm{C}$. Proteins were separated on $8 \%$ SDS-PAGE and blotted onto PVDF membrane (Roche, Mannheim, Germany). The blots were then blocked with 2\% BSA in TBS-T (0.05\% Tween-20 in $20 \mathrm{mM}$ Tris-buffered saline $\mathrm{pH} 7.2$, TBS-T) buffer for $1 \mathrm{~h}$ at RT, followed by staining overnight at $4{ }^{\circ} \mathrm{C}$ for phosphorylated receptor using a rabbit monoclonal anti-VEGFR2 phospho-tyrosine 1175 antibody (19A10, Cell Signaling) diluted in $2 \%$ BSA in TBS-T. As a loading control, the lower part of the blot was stained for actin overnight with a mouse monoclonal anti-actin antibody (ICN Biomedicals, Irvine, CA, USA). Afterwards, the blots were incubated with IRDye800CW goat anti rabbit secondary antibody (LI-COR) and IRDye800CW donkey anti mouse secondary antibody (LI-COR) for $1 \mathrm{~h}$ at RT. Bound antibody was visualized with an Odyssey infrared scanner at $800 \mathrm{~nm}$. Afterwards, blots were stripped using 1\% SDS and $25 \mathrm{mM}$ glycine pH 2, blocked with 2\% BSA and then incubated with rabbit anti-VEGFR2 monoclonal antibody (55B11, Cell Signaling) overnight at $4{ }^{\circ} \mathrm{C}$. After incubation with IRDye800CW goat anti rabbit secondary antibody, the total VEGFR2 was visualized at $800 \mathrm{~nm}$.

\section{Proliferation assay}

For the proliferation assay, MS1 cells were used as they showed better compatibility with the assay conditions among the different endothelial cell lines. Two thousand MS1 cells were seeded in 96 well plates in complete medium containing $10 \% \mathrm{FBS}$ and $1 \%$ penicillin/streptomycin. Next day, $10 \mathrm{nM}$ VEGF-A or different concentrations of the nanobodies $(1 \mathrm{nM}, 10 \mathrm{nM}, 100 \mathrm{nM})$ or the mixture of both in DMEM containing $0.2 \%$ FBS were added to the cells and incubated for $72 \mathrm{~h}$ at $37^{\circ} \mathrm{C}$. Cell viability was determined using AlamarBlue ${ }^{\circledR}$ reagent following the manufacturer's protocol (Bio- 
Rad). The percentage of changes in proliferation was calculated relative to the non-treated cells. The experiment was performed at least three times. The assay results have been shown as mean $\pm \mathrm{SD}$.

\section{In vitro PDT}

\section{Monoculture}

Total fluorescence of the associated NB-PS conjugates with the murine cell lines was determined $1 \mathrm{~h}$ after incubation at $37{ }^{\circ} \mathrm{C}$ as described earlier in section 4.8. Immediately after, cells were illuminated with $7 \mathrm{~mW} / \mathrm{cm}^{2}$ fluence rate for a total light dose of $20 \mathrm{~J} / \mathrm{cm}^{2}$ using a $690 \mathrm{~nm}$ diode laser through a $600 \mu \mathrm{m}$ optic fiber (Modulight, Tampere, Finland). After overnight incubation at $37^{\circ} \mathrm{C}$, AlamarBlue ${ }^{\circledast}$ reagent was added to the wells following the manufacturer's protocol (Bio-Rad) to assess cell viability. Cells with no light/no treatment were used as $100 \%$ viability and the percentage of viability calculated relative to the $100 \%$ viable cells. Data were analyzed with GraphPad Prism 8.0 software and presented as mean $\pm \mathrm{SD}$.

\section{$\underline{\text { Co-culture of endothelial and cancer cells }}$}

MS1 cells were co-seeded with OSC cells in 96 well plates (1 to 3 ratio and total number of 10000 cells/well) one day before the experiment. Next day, cells were incubated with different concentrations of 7D12-PS (targeting EGFR on OSC cells) or VEGFR2 targeting conjugates, or the mixture of both keeping the total concentrations of PS the same (e.g. $100 \mathrm{nM} 7 \mathrm{D} 12,100 \mathrm{nM}$ VM, or $50 \mathrm{nM}$ 7D12 + $50 \mathrm{nM} \mathrm{VM}$ ). After $1 \mathrm{~h}$ of incubation at $37{ }^{\circ} \mathrm{C}$, cells were illuminated, and the percentage of cell viability was determined the next day, as described above.

\section{Specificity of the nanobody-targeted PDT in co-culture setup}

MS1/OSC cells were treated with the NB-PS conjugates (100 nM 7D12-PS or VM2-PS, or $50 \mathrm{nM}+50 \mathrm{nM}$ PS of mixture) and $24 \mathrm{~h}$ after illumination, the medium was slowly removed, and PI ( $1 \mu \mathrm{g} / \mathrm{ml}$, Invitrogen) in PBS was added to the cells and incubated for $10 \mathrm{~min}$ at $37^{\circ} \mathrm{C}$. Triton $(1 \%)$ was used as a positive control to induce $100 \%$ cell death. The cells were imaged with an EVOS microscope and analyzed with ImageJ. 


\section{Results}

\section{Generation of high affinity anti-VEGFR2 nanobodies}

To obtain anti-VEGFR2 nanobodies, two llamas were injected with mouse VEGFR2 (mVEGFR2) protein and bEnd.3 cell-derived vesicles, followed by construction of the phage libraries as described in Materials and methods. Upon panning, 96 clones were screened for specific binding to VEGFR2 by means of a phage ELISA. Over 50 clones showed specific binding to VEGFR2 protein and after sequencing, ten nanobodies with unique sequences were selected for further characterization. After preliminary tests, seven nanobodies were excluded due to the low binding affinity to the target protein (data not shown) and the study was continued with the three most promising nanobodies, named VM1, VM2 and VM3. The nanobodies were produced in bacteria using a bioreactor and affinity purified from the periplasmic fractions with Äkta Xpress chromatography. The yield of production for each nanobody was approximately $60 \mathrm{mg}$ nanobody from $5 \mathrm{l}$ culture, and all nanobodies were obtained with excellent purity (Figure 1a,1). To determine binding affinity of the nanobodies to the target protein, binding assays were performed on the mVEGFR2 protein and the results indicated that all three nanobodies bound with high affinity to the target protein (Figure 1b). The apparent binding affinity of the nanobodies was $0.8 \pm 0.1 \mathrm{nM}$ for $\mathrm{VM} 1,1.2 \pm 0.2 \mathrm{nM}$ for $\mathrm{VM} 2$, and $1.5 \pm 0.1 \mathrm{nM}$ for VM3.

\section{Nanobodies remain high affinity binders after random conjugation}

As these nanobodies were meant to be used in PDT studies, we first tested the effect of the conjugation strategy on binding properties. For either microscopic or flow cytometry-based characterizations, all nanobodies were conjugated to Alexa647, while for PDT they were conjugated to the PS IRDye700DX. The conjugation of the nanobodies to PS/fluorophore occurred via random NHS-mediated coupling to primary amines in the nanobodies sequence. After purification, less than $2 \%$ free fluorophore was detected on SDS-PAGE gel for all nanobodies (Figure 1a,2). As for PS, in all conjugates less than $10 \%$ free PS was detected upon conjugate preparation (Figure 1a,3). Determination of DOC revealed that all nanobodies were on average conjugated to 1 molecule of fluorophore and 1.2 molecules of PS. To verify binding of the conjugates to the target protein, binding assays were performed with all the conjugates on mVEGFR2 protein. The apparent binding affinity of the NB-PS conjugates were: $9 \pm 0.8 \mathrm{nM}$ for VM1-PS, $7.4 \pm 0.2 \mathrm{nM}$ for VM2-PS, 
and $11 \pm 0.4 \mathrm{nM}$ for VM3-PS (Figure 1c), thus binding affinities remained in the low $\mathrm{nM}$ range after conjugation. Similar values were obtained with NB-Alexa647 conjugates (data not shown). To confirm the specificity of the nanobodies, binding of the NB-PS conjugates was performed on mouse VEGFR1 (mVEGFR1) and human VEGFR2 (hVEGFR2) proteins. The nanobodies did not bind to either mVEGFR1 or hVEGFR2, confirming their specificity towards mVEGFR2 (Figure 1c).

\section{Nanobodies associate with murine cell lines according to their VEGFR2 expression level}

Next to testing conjugates on purified protein, the specificity of the nanobodies was also verified on cells, using different techniques. For these, a panel of murine cell lines was employed, for which the expression of VEGFR2 was assessed using a commercial anti-VEGFR2-PE antibody with flow cytometry. Different levels of VEGFR2 expression were observed in the murine cell lines, i.e. bEnd.3> MS1> H5V, while NIH 3T3 are negative for VEGFR2 expression (Figure S1). We then investigated the association of the NB-PS conjugates with the cells in 96 well plates. Cells were incubated with different concentrations of the NB-PS conjugates for $1 \mathrm{~h}$ at $37{ }^{\circ} \mathrm{C}$ and the total fluorescence was plotted against concentration (Figure 1d). A clear correlation was observed between the total fluorescence intensity and the expression level of VEGFR2 on the cells, (i.e. bEnd.3 with the highest and NIH $3 \mathrm{~T} 3$ with the lowest association) indicating the nanobodies specifically bind VEGFR2. The same trend of association per cell was observed with the NB-Alexa647 conjugates through flow cytometry (Figure 1e,f), which is in agreement with the data obtained with the commercial antibody (Figure S1).

To further verify the specificity of the VEGFR2 nanobodies, bEnd.3 cells were pre-incubated with anti-VEGFR2-PE antibody or unconjugated nanobodies, followed by addition of the NB-PS conjugates. Addition of ten-times molar excess of the antibody or unlabeled nanobodies, resulted in a significant reduction in the cell-associated NB-PS conjugates, indicating competition of the nanobodies with anti-VEGFR2-PE antibody and with the unconjugated nanobodies $(* \mathrm{p}<0.05 ; * * \mathrm{p}<0.01 ; * * * \mathrm{p}<0.001 ; \mathrm{t}$ test, Figure 1g). 
(a)
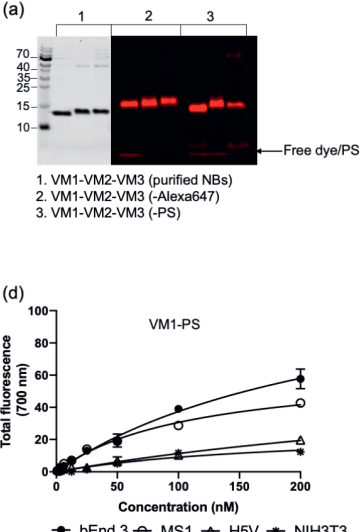

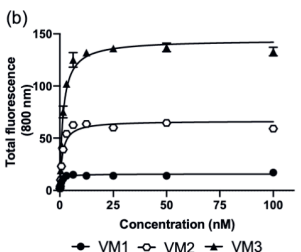

$\rightarrow$ VM1 - VM2 $\rightarrow$ VM3

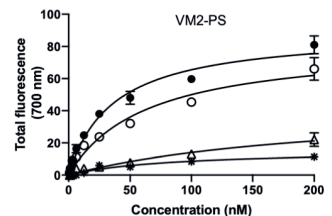

$\rightarrow$ bEnd. $3 \rightarrow \mathrm{MS} 1$ \& H5V * NIH3T3

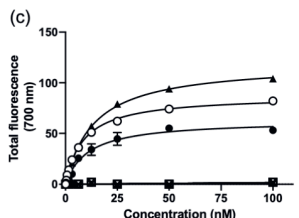

MVEGFR2: $\rightarrow$ VM1-PS $-0-$ VM2-PS $\div$ VM3-PS hVEGFR2: $\forall$ VM1-PS $*$ VM2-PS $\theta$ VM3-PS IVEGFR1: $\because$ VM1-PS VM2-PS $\#$ VM3-PS

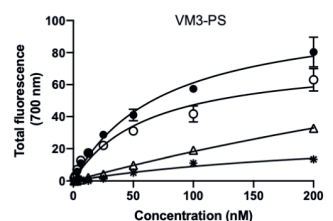

$\rightarrow$ bEnd. $3 \oplus \mathrm{MS} 1+\mathrm{H} 5 \mathrm{~V} * \mathrm{~N} \mathrm{NH} 3 \mathrm{~T}$

(e)
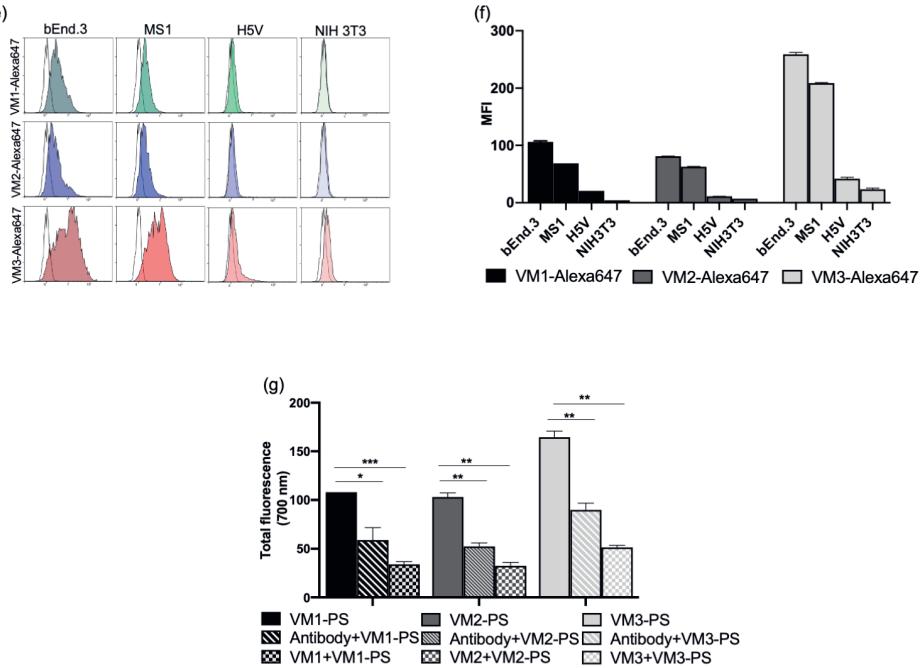

Figure 1. Purity and specificity of the NBs and NB-conjugates. (a) Purified NBs, NB-Alexa647 and NB-PS conjugates separated by SDS-PAGE. Free dye/PS is observed at the gel front (arrow) 1. Purified NBs after PageBlue staining (depicted in black). 2, 3. The fluorescence of Alexa647 and PS is detected at $700 \mathrm{~nm}$, respectively (depicted in red). (b) Binding of the unlabeled NBs to the mVEGFR2 protein detected by anti-VHH antibody. (c) Binding of the NB-PS conjugates to the mVEGFR2, hVEGFR2 and mVEGFR1 proteins. Total fluorescence of NB-PS bound to the protein was detected using an Odyssey infrared scanner at $700 \mathrm{~nm}$. (d) Total fluorescence intensity of cell bound/internalized NB-PS conjugates on the murine cell lines after $1 \mathrm{~h}$ incubation at $37{ }^{\circ} \mathrm{C}$. (e-f) Fluorescence of NB-Alexa647 conjugates detected by flow cytometry. The murine cell lines were incubated with the conjugates for $1 \mathrm{~h}$ at $37^{\circ} \mathrm{C}$ followed by trypsinization and FACS analysis. Mean fluorescent intensity (MFI) obtained from flow cytometry. Data shown as mean $\pm \mathrm{SD}$. (g) In vitro competition experiments of the NB-PS conjugates tested on bEnd.3 cells in the presence or absence of ten-times excess of anti-VEGFR2 antibody or unconjugated NBs. Total fluorescence intensity of the associated NB-PS conjugates was detected by an Odyssey infrared scanner at $700 \mathrm{~nm}(* \mathrm{p}<0.05$; $* * \mathrm{p}<0.01 ; * * * \mathrm{p}<0.001 ; \mathrm{t}$ test $)$. 


\section{Nanobodies are internalized after $1 \mathrm{~h}$ incubation under static and flow conditions}

In order to investigate the association of the nanobodies with VEGFR2 on cells (Figure 1d) in more detail, confocal imaging was performed after $1 \mathrm{~h}$ incubation at $37{ }^{\circ} \mathrm{C}$ under static and flow conditions. As control, the expression/localization of VEGFR2 in the murine cell lines was verified using a commercial antibody (Figure S2). Cells incubated with the antibody showed perinuclear VEGFR2 staining, with varying intensities correlating with the level of VEGFR2 expression (Figure S1), and no signal was observed in the NIH 3 T3 cells, which lack VEGFR2 expression. A similar pattern was observed with the NB-Alexa647 conjugates (Figure 2a). All the conjugates showed association with the VEGFR2 expressing cells, while none of them associated with the negative cells (NIH 3T3, Figure 2a).

The interaction of the nanobodies with bEnd.3 cells was investigated after incubation under flow conditions using ibidi pump system ${ }^{\circledR}$ (Figure 2b) to assess whether the nanobodies had sufficient affinity to associate with endothelial cells under flow conditions. Incubation under flow led to similar images of NB-Alexa647 conjugates associated with bEnd.3 cells (Figure 2c). As could be expected from association experiments conducted under flow, the association of nanobodies with cells was less pronounced than in static conditions. Nevertheless, both VM2 and VM3 were clearly taken up by bEnd. 3 cells. 
(a)

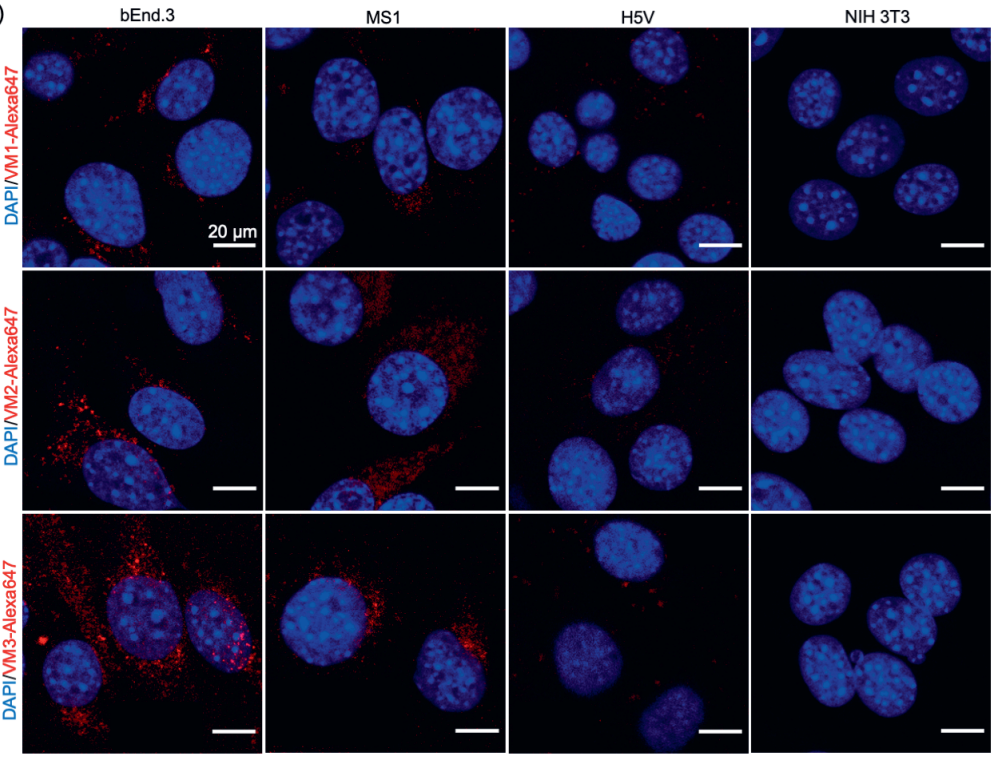

(b)

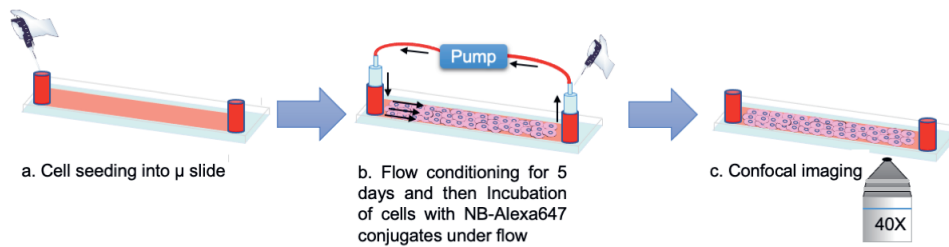

(c)

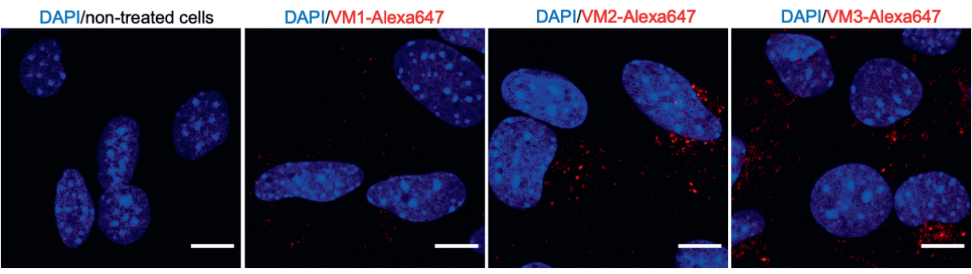

Figure 2. Association of the NB-Alexa647 conjugates with the murine cells after $1 \mathrm{~h}$ incubation under static and flow conditions. (a) Confocal images of the cells incubated with the NB-Alexa647 conjugates for $1 \mathrm{~h}$ at $37^{\circ} \mathrm{C}$. (b) Schematic representation of the experimental setup for the flow study using ibidi pump system ${ }^{\circledR}$. bEnd.3 cells were seeded into the $\mu$ slides on day 1 and kept under a unidirectional flow for 5 days. Cells were incubated with the NB-Alexa647 conjugates for $1 \mathrm{~h}$ under flow, and slides were subsequently prepared for confocal imaging. (c) Representative images obtained with a confocal microscope after incubation of the conjugates with bEnd.3 cells under flow (shear rate $300 / \mathrm{sec}$ ). Nuclei stained in blue and the conjugates shown in red. 


\section{Nanobodies are non-agonists and inhibit VEGF-A induced proliferation of MS1 cells}

Incubation of serum-starved MS1 cells with VEGF-A for 15 min at $37{ }^{\circ} \mathrm{C}$ showed increased phosphorylation of tyrosine 1175 of VEGFR2 (Figure 3a). Anti-VEGFR2 nanobodies did not stimulate VEGFR2 phosphorylation, indicating that the nanobodies do not act as receptor agonists (Figure 3b). Interestingly, anti-VEGFR2 nanobodies showed antiproliferative effects in the presence or absence of VEGF-A. Treatment of MS1 cells in low serum medium containing $10 \mathrm{nM}$ VEGF-A over a 3-day period resulted in about $40 \%$ increase in cell proliferation relative to the non-treated cells. In general, nanobodies alone did not stimulate cell proliferation, though a slight proliferation is suggested at the lowest concentration of the nanobodies $(1 \mathrm{nM})$. As this is observed after a 3-day incubation, it is likely not relevant in the context of PDT. In addition, these nanobodies inhibited VEGFstimulated proliferation in a dose dependent manner, which suggests competition of the nanobodies with VEGF-A (Figure 3c). This is in line with the result of the competition assay in which the addition of 10x or 100x excess of VEGF resulted in a significant reduction in the protein-bound NB-PS conjugates (Figure S3). 
(a)

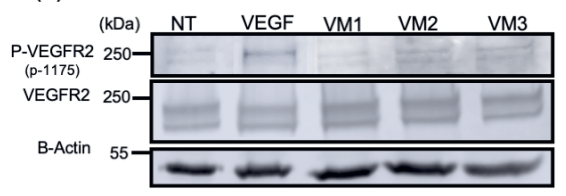

(c)

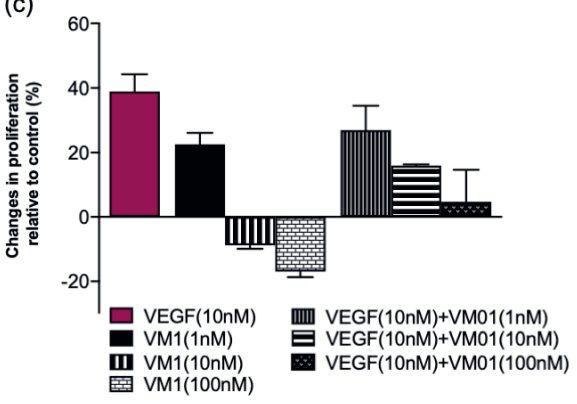

(b)
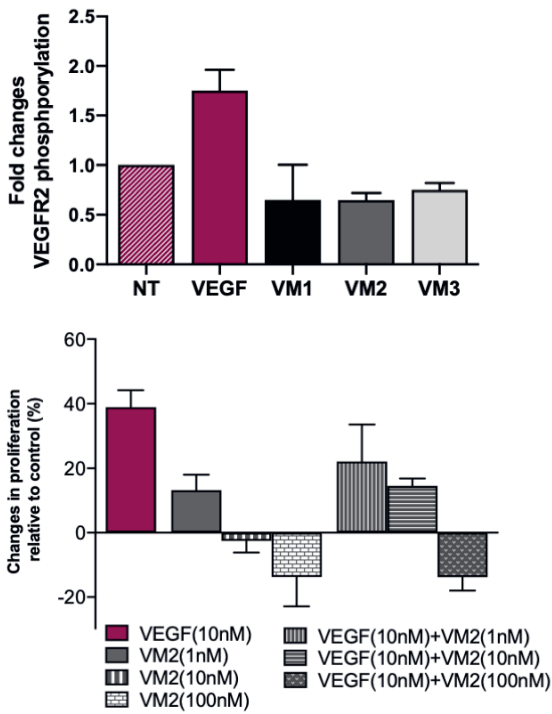

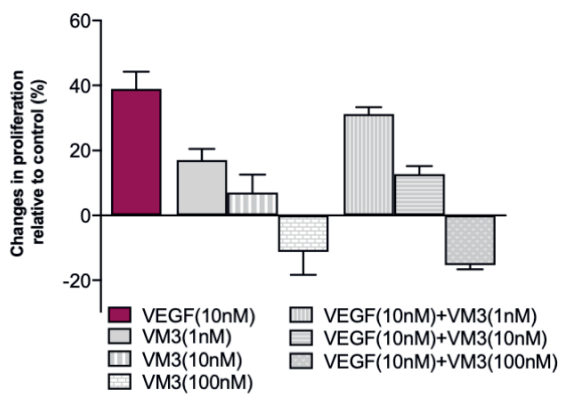

Figure 3. Anti-VEGFR2 NBs blocked VEGF-induced proliferation and did not act as receptor agonists. (a) VEGF-A $(50 \mathrm{nM})$ or NBs $(1 \mu \mathrm{M})$ were added to the serum-starved MS1 cells and incubated for $15 \mathrm{~min}$. VEGFR2 phosphorylation was measured in the total cell lysates by western blotting. The upper panel shows staining of phosphorylated tyrosine 1175 of VEGFR2. The middle panel shows staining of total VEGFR2. The lower panel shows staining of actin. (b) Fold changes of P-VEGFR2 in MS1 cells treated with VEGF or nanobodies relative to the non-treated cells (NT). (c) MS1 cells treated with VEGF-A/NBs or both for $72 \mathrm{~h}$ followed by viability assay using AlamarBlue ${ }^{\circledR}$ reagent. Data are presented as \% changes. in cell proliferation relative to the non-treated cells (mean $\pm \mathrm{SD})$. 


\section{VEGFR2 targeted NBs-PS conjugates are potent and specific PDT agents}

The ability of the NB-PS conjugates to kill VEGFR2 expressing cells was assessed by measuring cell viability one day after illumination of NB-PS associated cells (the association is referred as pulse and described in Figure 1d). The cytotoxicity induced correlated well with the level of VEGFR2 expression: i.e. bEnd.3 and MS1 were effectively killed, and, importantly, cells with no VEGFR2 expression were not affected (Figure 4, corresponding $\mathrm{EC}_{50}$ values are listed in Table 1). Among the NB-PS conjugates, the strongest cytotoxicity was induced by VM2-PS and VM3-PS.
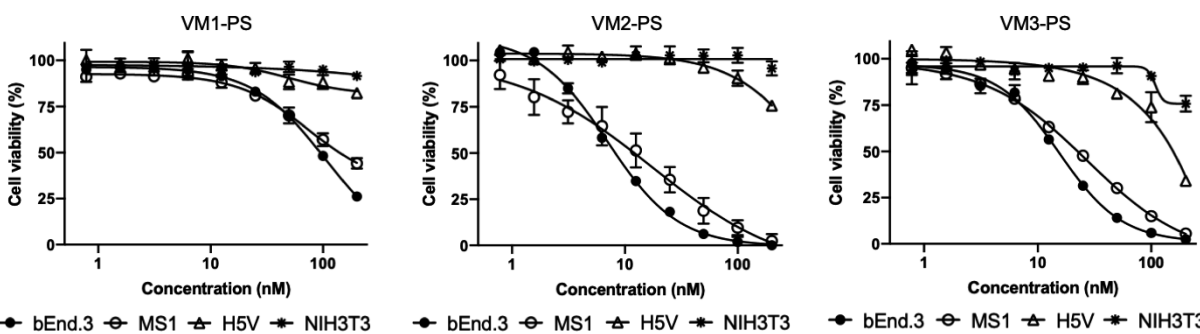

Figure 4. In vitro nanobody-targeted PDT in monoculture. The percentage of cell viability $24 \mathrm{~h}$ after illumination relative to the non-treated cells. Cells were incubated with different concentrations of the NB-PS conjugates for $1 \mathrm{~h}$ at $37{ }^{\circ} \mathrm{C}$, and illuminated with $7 \mathrm{~mW} / \mathrm{cm}^{2}$ fluence rate and a total light dose of $20 \mathrm{~J} / \mathrm{cm}^{2}$ using a $690 \mathrm{~nm}$ diode laser through a $600 \mu \mathrm{m}$ optic fiber.

Table 1. $\mathrm{EC}_{50}$ values obtained from nanobody-targeted PDT of two murine endothelial cell lines.

\begin{tabular}{ccc}
\hline NB-PS & $\begin{array}{c}\text { bEnd.3 } \\
\mathrm{EC}_{50} \pm \mathrm{SD}(\mathrm{nM})\end{array}$ & $\begin{array}{c}\mathrm{MS} 1 \\
\mathrm{EC}_{50} \pm \mathrm{SD}(\mathrm{nM})\end{array}$ \\
\hline $\mathrm{VM} 1$ & $92 \pm 1$ & $155 \pm 1.1$ \\
\hline $\mathrm{VM} 2$ & $8.6 \pm 1$ & $11 \pm 1.1$ \\
\hline $\mathrm{VM} 3$ & $14 \pm 1$ & $21 \pm 1$ \\
\hline
\end{tabular}

\section{Cytotoxicity is enhanced by combining the EGFR and VEGFR2 targeted NB- PS}

To improve PDT efficacy, the combination of endothelial and cancer cell targeting is here explored. For this, co-culture experiments were conducted with the human oral squamous cell carcinoma (OSC) and murine endothelial cells (MS1). MS1/OSC co-cultures were incubated with different concentrations of the VEGFR2 targeted nanobodies, the EGFR targeted nanobody 7D12-PS (described in $[16,19]$ ), or their combination. In order to 
perform a head-to-head comparison of cytotoxicity induced after PDT, the total concentration of PS conjugate was the same for the combination and the single conjugates (the DOC was used for this correction). When the individual NB-PS conjugates were used to target VEGFR2 or EGFR, we couldn't obtain low $\mathrm{nM} \mathrm{EC}_{50}$ values, as we did with the monocultures (Table 1). In the contrary, $\mathrm{EC}_{50}$ were above the highest tested concentration. However, when the combination of 7D12-PS and anti-VEGFR2 nanobodies were incubated in the co-culture setup, cellular cytotoxicity significantly increased compared to the individual treatments (Figure 5a, for concentration $\geq 50 \mathrm{nM}$, *p $<0.05$ for VM1, and **p $<0.01$ for VM2 and VM3, t test), with $\mathrm{EC}_{50}$ values of $103 \pm 1 \mathrm{nM}$ for $\mathrm{VM} 1,72 \mathrm{nM} \pm 1.1$ for VM2 and $60 \mathrm{nM} \pm 1.2$ for VM3. These results indicate the combination of both conjugates increased effectiveness of nanobody-targeted PDT.

Furthermore, the combination seems to be more potent than the sum of the separate effects: as an example, $100 \mathrm{nM}$ of combined VM2-PS and 7D12-PS (50 nM of each), resulted in $56 \pm 0.8 \%$ cell death, while $50 \mathrm{nM}$ of 7D12-PS caused $20 \pm 2.4 \%$ and

VM2-PS $14 \pm 1.5 \%$ cell death (sum would be approximately $35 \%$ cell death, $* \mathrm{p}<0.05$ ).

Overall, the results suggest a synergistic cytotoxicity resulting from the combination of the nanobodies, as higher cytotoxicity was obtained with the combined nanobodies compared with the sum of the two separate treatments.

\section{MS1 or OSC cells are specifically killed in co-cultures}

The specific cell killing by nanobody-targeted PDT in the co-culture setup was confirmed by staining dead cells with PI, after nanobody-targeted PDT with each single conjugate. In the co-culture setup, MS1 and OSC cells were distinguished by different morphology: MS1 appear as elongated cells (red arrows, Figure 5b), while OSC appear as round cells grown in patches (black arrows, Figure 5b). When MS1/OSC cells were incubated with VM2-PS or 7D12-PS separately, only MS1 or OSC cells were killed, respectively. Treatment of MS1/OSC cells with the combination of VM2-PS and 7D12-PS resulted in more extensive cell cytotoxicity, killing almost every cell (Figure 5b). 
(a)
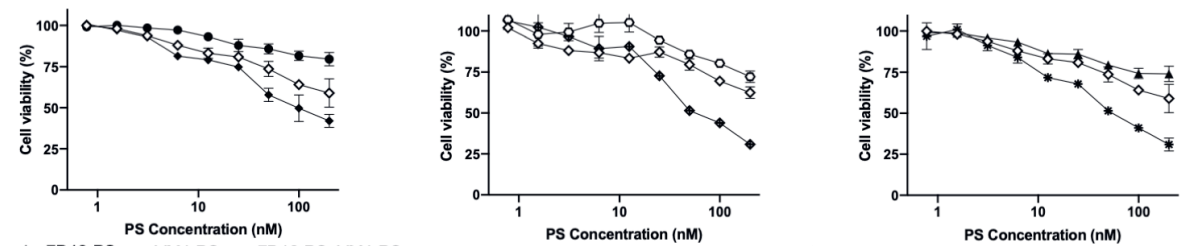

$\diamond$ 7D12-PS $\bullet$ VM1-PS $\rightarrow$ 7D12-PS+VM1-PS

$\diamond 7 D 12-P S-0$ VM2-PS $\leftarrow 7 D 12-P S+V M 2-P S$

$\diamond 7 D 12-P S$ — VM3-PS * 7D12-PS+VM3-PS
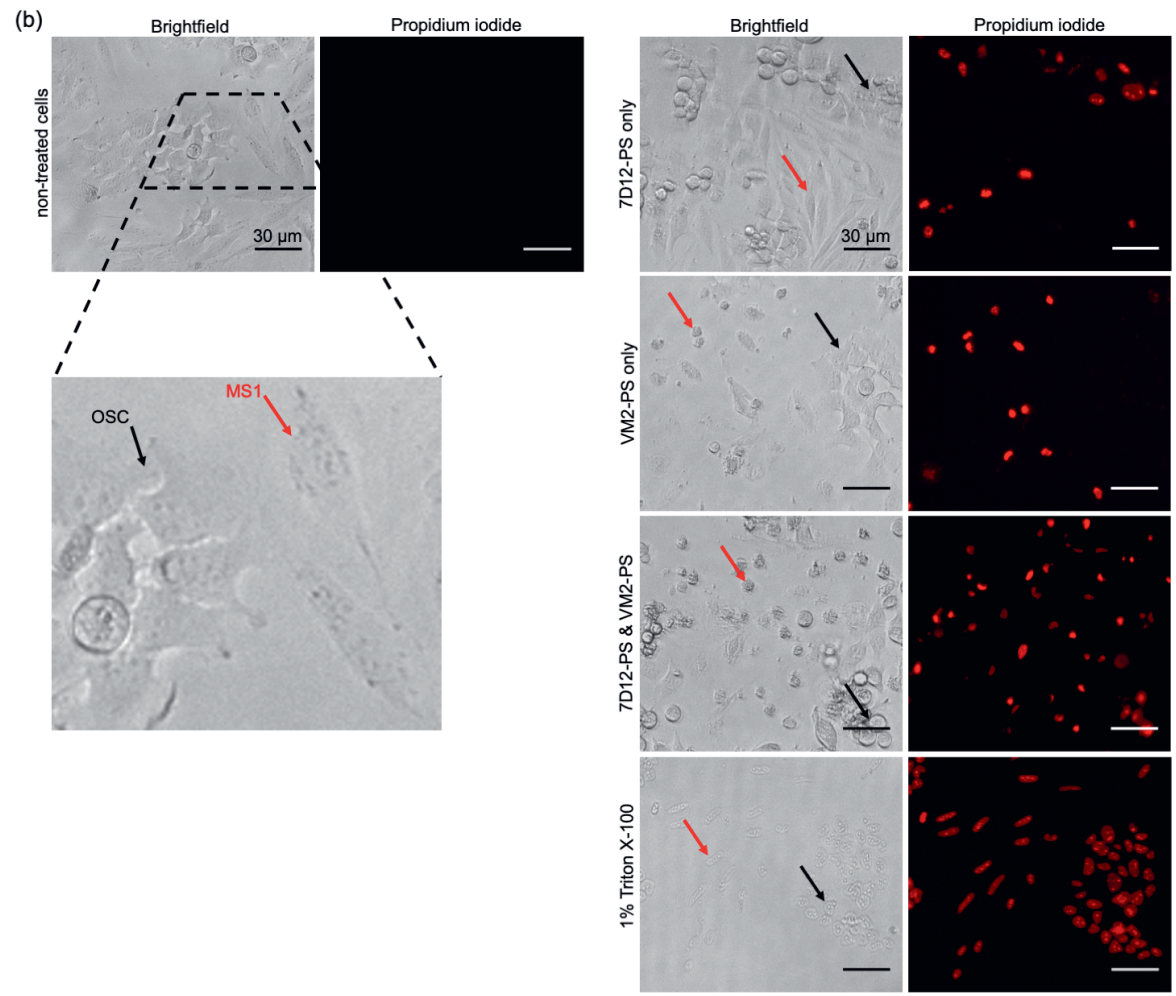

Figure 5. In vitro nanobody-targeted PDT in co-culture of endothelial and cancer cells. (a) The percentage of cell viability $24 \mathrm{~h}$ after illumination relative to the non-treated cells. Murine endothelial cells (MS1) and human cancer cells (OSC) were co-seeded with 1:3 ratio and incubated with different concentrations of VM-PS conjugates targeting VEGFR2 on endothelial cells, and 7D12-PS targeting EGFR on cancer cells for $1 \mathrm{~h}$ at $37{ }^{\circ} \mathrm{C}$, then illuminated with $7 \mathrm{~mW} / \mathrm{cm}^{2}$ fluence rate and a total light dose of $20 \mathrm{~J} / \mathrm{cm}^{2}$ using a $690 \mathrm{~nm}$ diode laser through a $600 \mu \mathrm{m}$ optic fiber. (b) MS1/OSC co-culture treated with VM2-PS (targeting VEGFR2 on MS1) or 7D12-PS (targeting EGFR on OSC cells) or the mixture of both. Dead cells were stained with propidium iodide $24 \mathrm{~h}$ after illumination. MS1 and OSC cells shown with red and black arrows, respectively. 


\section{Discussion}

Efficacy of targeted PDT can be hampered by heterogeneity of target expression and/or moderate/low target expression levels. To circumvent these, we explored the possibility of combined targeting of endothelial and cancer cells in vitro, as a proof of concept. For this, we developed nanobodies targeting mouse VEGFR2 which is overexpressed mainly on tumor vasculature. After thorough characterization of these nanobodies, we showed that the conjugates are potent PDT agents, specifically killing cells expressing VEGFR2. Moreover, dual targeting of endothelial and cancer cells, using nanobody-photosensitizer conjugates targeting VEGFR2 and EGFR, respectively, showed improved efficacy.

Our mVEGFR2 targeting nanobodies demonstrated high binding affinity to the target protein with single-digit nanomolar dissociation constants $\left(\mathrm{K}_{\mathrm{D}}\right)$. Interestingly these nanobodies showed different maximum binding values $\left(\mathrm{B}_{\max }\right)$. This might be caused by a. the different epitope accessibility of the nanobodies and/or b. the indirect recognition of the binding of the nanobodies with anti-VHH antibody, as these nanobodies are from three different sequence families. With directly labeled nanobodies, which remained high affinity binders, the difference in $\mathrm{B}_{\max }$ was smaller among the different nanobodies, supporting the second explanation. To verify the specificity of the nanobodies, different techniques were applied on both protein and cellular levels. On protein level, we have shown that all nanobodies only bound to mVEGFR2 and not hVEGFR2 or mVEGFR1. Among the VEGFR family members, mVEGFR1 was employed because its extracellular domain shares the highest sequence homology (43\%) with the one of VEGFR2 [31]. On the cellular level, binding affinity of the nanobodies to the endothelial cells was not determined as endothelial cells detach from the well plates when incubated at $4{ }^{\circ} \mathrm{C}$. However, when conducting the assays at $37{ }^{\circ} \mathrm{C}$, the amount of NB-PS conjugates associated with the murine cell lines correlated well with the expression levels of VEGFR2. This was also verified by flow cytometry analysis, indicating specificity of these nanobodies for mVEGFR2. Other high affinity nanobodies have been described to bind VEGFR2, however only the human variant [32]. The fact that these nanobodies only bind mouse VEGFR2 will enable dedicated studies on the effects of treatment on tumor vasculature. For translational purposes, cross reactive nanobodies could be obtained through dedicated selections. 
In our microscopic investigations, internalization and perinuclear accumulation of the NB-Alexa647 conjugates in the cells were observed by confocal imaging, which is in agreement with the results obtained with the commercial anti-VEGFR2 antibody. These results are also in agreement with the findings of other groups where perinuclear staining of VEGFR2 was shown after short incubation with anti-VEGFR2 antibody [33,34]. This perinuclear localization of monovalent nanobodies or antibody is likely a result of constitutive internalization of the receptor, as Gampel and co-workers showed that a significant portion of VEGFR2 undergoes constitutive endocytosis [33]. In nanobodytargeted PDT, the damage is inflicted where the nanobody-PS is located, thus here damage will be mostly perinuclear/intracellular. In addition to the static condition, we also explored the association of the NB-Alexa647 conjugates to the cells under flow condition. In in vivo condition, endothelial cells are exposed to the constant blood flow which might change and reduce the interaction of the nanobodies with the cells. Therefore, we decided to investigate whether incubating bEnd.3 cells with NB-Alexa674 conjugates for $1 \mathrm{~h}$ under unidirectional flow would still enable cell association and internalization. The setup we used for the experiments under flow is particularly appropriate to study the interaction of the therapeutics with endothelial cells and can, to some extent, predict the in vivo association of the nanobodies targeting endothelial cells when administered systemically [35]. These experiments were conducted at a shear rate of $300 / \mathrm{sec}$, which is comparable to the shear rate reported for the human carotid artery. The alignment of $\mathrm{F}$ actin cytoskeleton fibers suggests that cells were shear-adapted (Figure S4), in agreement with other studies [35]. Among the NB-Alexa647 conjugates, VM2 and VM3 were associated with cells. VM1 on the other hand was barely associated with cells, despite showing cell association under the static condition. These results are in agreement with a recently published study showing that the uptake of nanoparticles into endothelial cells is decreased by increasing the flow rate $[35,36]$.

Although nanobody-targeted PDT protocols are conducted in a short period of time, i.e. light is applied 1-2 h post intravenous injection, and involve a single treatment session, we considered it important to assess the agonistic potential of these nanobodies. The phosphorylation assay proved that VEGFR2 is not activated by the nanobodies, ruling out any agonistic activity. The nanobodies did not induce cell proliferation when used at $10 \mathrm{nM}$ or $100 \mathrm{nM}$. However, in lower concertation $(1 \mathrm{nM})$, cell proliferation was slightly 
induced by the nanobodies over the period of 3-day incubation. This likely will not cause any problem in vivo for two reasons: a. nanobodies have shorter half-life and will be cleared within a day, and $\mathrm{b}$. the concentration of nanobodies used for in vivo study will be likely higher than $1 \mathrm{nM}$. Interestingly, the nanobodies also inhibited VEGF-stimulated proliferation in low nanomolar concentrations, suggesting competition of the nanobodies with VEGF (confirmed with a competition assay in Figure S3) and inhibition of VEGF signaling pathways in vitro. However, this inhibition might not be observed in the tumor microenvironment as the concentration and the affinity of the ligand to the receptor is higher than that of nanobodies. Therefore, the competition of the nanobodies with VEGF should be verified in physiological and more complex conditions.

Among the NB-PS conjugates with similar DOC, VM2-PS and VM3-PS induced nearly $100 \%$ cell death in bEnd. 3 and MS1 cells when tested at $100 \mathrm{nM}$. $\mathrm{EC}_{50}$ values were calculated in nanomolar ranges, indicating that the NB-PS conjugates are potent PDT agents. Importantly, non-VEGFR2 expressing cells were not affected after PDT, providing support for the specificity of nanobody-targeted PDT approach. Besides VEGFR2, there are other receptors on tumor vasculature which can be used for targeted VTP, as recently reviewed [37]. Frochot and colleagues reported integrin $\alpha v \beta 3$ targeted peptides for VTP. Phototoxicity induced by cyclic RGD peptide-PS conjugate at $1 \mu \mathrm{M}$ was assessed after $24 \mathrm{~h}$ incubation with HUVEC overexpressing $\alpha \mathrm{v} \beta 3$ cells and $50 \%$ growth inhibition $\left(\mathrm{LD}_{50}\right)$ value was reported $3.1 \mathrm{~J} / \mathrm{cm}^{2}$ [38]. In another study, Neuropilin-1 targeted heptapeptide was conjugated to tetraphenylchlorin (TPC) and PDT efficacy was determined in HUVEC $24 \mathrm{~h}$ after incubation with $100 \mathrm{nM}$ conjugate or free PS. Treatment with free PS showed little cytotoxicity, whereas cells treated with the peptide-PS conjugate had 10.4-fold lower viability. $\mathrm{LD}_{50}$ value was reported $0.47 \pm 0.23 \mathrm{~J} / \mathrm{cm}^{2}$ and $4.9 \pm 0.64 \mathrm{~J} / \mathrm{cm}^{2}$ for the conjugate and free PS respectively [39]. Comparison between these studies is difficult due to different conditions employed, however the low $\mathrm{nM} \mathrm{EC}_{50}$ values presented here certainly encourage further exploration of anti-VEGFR2 conjugates in a preclinical setting.

For the co-culture setup, MS1 cells with moderate level of VEGFR2 expression and, OSC cells with moderate level of EGFR expression were selected. In particular for EGFR, our previous research has shown that organoids grown from tumors of patients with head and neck cancers possess low/moderate EGFR levels, compared to cell lines traditionally used in EGFR research (e.g. A431 cell line) [40]. When EGFR and VEGFR2 
targeting nanobodies were applied in combination, noting the particular set-up used here with human cancer cells and murine endothelial cells, no interference was observed of either nanobody-PS (VM2-PS or 7D12-PS) on the association of the other nanobody-PS (7D12-PS or VM2-PS) with cells (Figure S5). After illumination, the combined treatment was significantly more effective than the individual treatments. Importantly, $\mathrm{EC}_{50}$ values were still in nanomolar level when VEGFR2 and EGFR were simultaneously targeted. For the individual treatments, when endothelial and cancer cells were co-seeded, obviously part of cells were not killed due to the lack of target expression, thus the higher $\mathrm{EC}_{50}$ values when compared to those obtained in the monoculture setup. Importantly, when nanobodies were applied separately, one cell population was specifically killed (Figure 5b), whereas when the combination of the nanobodies was used, cytotoxicity was significantly higher. Interestingly, the cytotoxicity is even higher than the sum of the separate effects, which suggests synergism. The higher cytotoxicity induced by combined nanobodies could be due to the overall higher amount of reactive oxygen/nitrogen species produced during illumination, which can affect adjacent cells $[1,41]$. These results support our hypothesis that simultaneous targeting of endothelial and cancer cells can improve nanobody-targeted efficacy. Follow-up studies will aim at the efficacy evaluation of the conjugates in mice with human tumors. In this context, the cancer cell targeted nanobody-PS conjugates are expected to efficiently distribute through tumors and allow light application 1-2 $\mathrm{h}$ after intravenous injection $[19,20]$, while these VEGFR2 nanobody-PS conjugates are expected to interact and be taken up by the (mouse) tumor vasculature. Of note, the time point post injection at which light will be applied will be carefully determined, aiming at the most practical and effective protocol.

Intratumoral heterogeneity is one of the leading causes of therapeutic resistance and treatment failure and one of the main reasons for poor overall survival in cancer patients. In addition, it has been shown that targets against which therapeutics are available, are not expressed in a homogeneous manner in tumor tissues $[21,22,40]$. Therefore, combination approaches that target cancer cells and tumor vasculature can potentiate the therapeutic response [42]. Further preclinical studies are needed to investigate the vascular effects of this conjugates and the feasibility for clinical application, to improve selectivity and efficacy of cancer treatment. 


\section{Conclusion}

We have reported the successful development of nanobodies targeting mouse VEGFR2 which demonstrated high association with cells under flow and static conditions. In addition, the nanobody-photosensitizer conjugates were shown to be potent and specific PDT agents. Importantly, we improved the efficacy of nanobody-targeted PDT in coculture of endothelial and cancer cells when treating the cells with dual targeted nanobodyphotosensitizer conjugates. To our knowledge, this is the first time nanobodies targeting mouse VEGFR2 were developed with the potential to be applied for preclinical validation of VTP. Further in vivo studies are needed to verify the synergistic/additive effects of the dual targeted PDT.

\section{Acknowledgments}

The authors would like to thank Naomi Parr and Dr. Marcel Fens at University Medical Center Utrecht for the technical assistance to setup the ibidi pump flow system. Dr. Henriette S. de Bruijn and Dr. Dominic J. Robinson at Rotterdam Erasmus Medical Center are greatly acknowledged for insightful discussions. This work was supported by the European Research Council (ERC) under the European Union's Horizon 2020 research and innovation program (grant agreement No 677582).

\section{References}

1. Packer, L.; Cadenas, E., Methods in enzymology. Nitric Oxide, Part Eled. by Lester Packer. Pt. E. Gulf Professional Publishing: 2005; Vol. 301, 207-214.

2. $\quad$ Fong, W.P.; Yeung, H.Y.; Lo, P.C.; Ng, D.K.P. Photodynamic therapy. In Handbook of Photonics for Biomedical Engineering; Springer Netherlands, 2017; pp. 657-681.

3. Dougherty, T.J.; Gomer, C.J.; Henderson, B.W.; Jori, G.; Kessel, D.; Korbelik, M.; Moan, J.; Peng, Q.; Dougherty, T.J.; Henderson, B.W. Photodynamic Therapy. J. Natl. Cancer inst. 1998, 90, 889-905.

4. Ackroyd, R.; Kelty, C.; Brown, N.; Reed, M. The History of Photodetection and Photodynamic Therapy. Photochem 2001, 74, 656-669.

5. Allison, R.R.; Sibata, C.H. Oncologic photodynamic therapy photosensitizers: A clinical review. Photodiagn. photodyn. 2010, 7, 61-75.

6. Maeda, H.; Nakamura, H.; Fang, J. The EPR effect for macromolecular drug delivery to solid tumors: Improvement of tumor uptake, lowering of systemic toxicity, and distinct tumor imaging in vivo. Adv. Drug Deliv. Rev. 2013, 65, 71-79.

7. Sibani, S.A.; McCarron, P.A.; Woolfson, A.D.; Donnelly, R.F. Photosensitiser delivery for photodynamic therapy. Part 2: Systemic carrier platforms. Expert Opin Drug Del 2008, 5, $1241-1254$.

8. Mazor, O.; Brandis, A.; Plaks, V.; Neumark, E.; Rosenbach-Belkin, V.; Salomon, Y.; Scherz, A. WSTI 1, A Novel Water-soluble Bacteriochlorophyll Derivative; Cellular Uptake, 
Pharmacokinetics, Biodistribution and Vascular-targeted Photodynamic Activity Using Melanoma Tumors as a ModelT. Photochem. 2005, 81, 342-351.

9. Olivo, M.; Bhuvaneswari, R.; Lucky, S.S.; Dendukuri, N.; Thong, P.S.P. Targeted therapy of cancer using photodynamic therapy in combination with multi-faceted anti-tumor modalities. Pharmaceuticals 2010, 3, 1507-529.

10. Gill, I.S.; Azzouzi, A.R.; Emberton, M.; Coleman, J.A.; Coeytaux, E.; Scherz, A.; Scardino, P.T. Randomized Trial of Partial Gland Ablation with Vascular Targeted Phototherapy versus Active Surveillance for Low Risk Prostate Cancer: Extended Followup and Analyses of Effectiveness. J. Urol. 2018, 200, 786-793.

11. Richards, D.A. Exploring alternative antibody scaffolds: Antibody fragments and antibody mimics for targeted drug delivery. Drug Discov. Today Technol. 2018, 30, 35-46.

12. Hamers-Casterman, C.; Atarhouch, T.; Muyldermans, S.; Robinson, G.; Hammers, C.; Songa, E.B.; Bendahman, N.; Hammers, R. Naturally occurring antibodies devoid of light chains. Nature 1993, 363, 446-448.

13. Oliveira, S.; van Dongen, G.A.M.S.; Walsum, M.S.; Roovers, R.C.; Stam, J.C.; Mali, W.; van Diest, P.J.; van Bergen en Henegouwen, P.M.P. Rapid Visualization of Human Tumor Xenografts through Optical Imaging with a Near-Infrared Fluorescent Anti-Epidermal Growth Factor Receptor Nanobody. Mol. Imaging 2012, 11, 33-46.

14. Kijanka, M.; Warnders, F.-J.; el Khattabi, M.; Lub-de Hooge, M.; van Dam, G.M.; Ntziachristos, V.; de Vries, L.; Oliveira, S.; van Bergen en Henegouwen, P.M.P. Rapid optical imaging of human breast tumour xenografts using anti-HER2 VHHs site-directly conjugated to IRDye800CW for image-guided surgery. European Journal of Nuclear Medicine and Molecular Imaging 2013, 40, 1718-1729.

15. Muyldermans, S. Nanobodies: Natural Single-Domain Antibodies. Annu. Rev. Biochem 2013 $82,775-797$.

16. Heukers, R.; van Bergen en Henegouwen, P.M.P.; Oliveira, S. Nanobody-photosensitizer conjugates for targeted photodynamic therapy. Nanomed. Nanotechnol. 2014, 10, 14411451 .

17. Heukers, R.; Mashayekhi, V.; Ramirez-Escudero, M.; de Haard, H.; Verrips, T.C.; van Bergen en Henegouwen, Paul.M.P.; Oliveira, S. VHH-Photosensitizer Conjugates for Targeted Photodynamic Therapy of Met-Overexpressing Tumor Cells. Antibodies 2019, 8 , $1-13$.

18. de Groof, T.W.M.; Mashayekhi, V.; Fan, T.S.; Bergkamp, N.D.; Sastre Toraño, J.; van Senten, J.R.; Heukers, R.; Smit, M.J.; Oliveira, S. Nanobody-Targeted Photodynamic Therapy Selectively Kills Viral GPCR-Expressing Glioblastoma Cells. Mol. Pharm. 2019, $16,3145-3156$.

19. van Driel, P.B.A.A.; Boonstra, M.C.; Slooter, M.D.; Heukers, R.; Stammes, M.A.; Snoeks, T.J.A.; de Bruijn, H.S.; van Diest, P.J.; Vahrmeijer, A.L.; van Bergen en Henegouwen, P.M.P.; et al. EGFR targeted nanobody-photosensitizer conjugates for photodynamic therapy in a pre-clinical model of head and neck cancer. J. Controlled Release 2016, 229, 93-105.

20. Deken, M.M.; Kijanka, M.M.; Beltrán Hernández, I.; Slooter, M.D.; de Bruijn, H.S.; van Diest, P.J.; van Bergen en Henegouwen, P.M.P.; Lowik, C.W.G.M.; Robinson, D.J.; Vahrmeijer, A.L.; et al. Nanobody-targeted photodynamic therapy induces significant tumor regression of trastuzumab-resistant HER2-positive breast cancer, after a single treatment session. J. Controlled Release 2020, 323, 269-281.

21. Dagogo-Jack, I.; Shaw, A.T. Tumour heterogeneity and resistance to cancer therapies. Nat. Rev. Clin. Oncol. 2018, 15, 81-94.

22. Ramón y Cajal, S.; Sesé, M.; Capdevila, C.; Aasen, T.; de Mattos-Arruda, L.; Diaz-Cano, S.J.; Hernández-Losa, J.; Castellví, J. Clinical implications of intratumor heterogeneity: challenges and opportunities. J. Mol. Med. 2020, 98, 161-177.

23. Kijanka, M.M.; van Brussel, A.S.A.; van der Wall, E.; Mali, W.P.T.M.; van Diest, P.J.; van Bergen en Henegouwen, P.M.P.; Oliveira, S. Optical imaging of pre-invasive breast cancer 
with a combination of VHHs targeting CAIX and HER2 increases contrast and facilitates tumour characterization. EJNMMI Res. 2016, 6, 1-13.

24. Shibuya, M. VEGFR and type-V RTK activation and signaling. Cold Spring Harb. Perspect. Biol. 2013, 5.

25. Ferrara N VEGF and the quest for tumour angiogenesis factors. Nat. Rev. 2002, 2, 795-803.

26. Ferrara, N. Vascular endothelial growth factor: Basic science and clinical progress. Endocr. Rev. 2004, 25, 581-611.

27. Liu, Z.; Qi, L.; Li, Y.; Zhao, X.; Sun, B. VEGFR2 regulates endothelial differentiation of colon cancer cells. BMC Cancer 2017, 17 .

28. Shibuya, M. Vascular endothelial growth factor and its receptor system: Physiological functions in angiogenesis and pathological roles in various diseases. J. Biochem. 2013, 153, 13-19.

29. Kaplan, M.; Narasimhan, S.; de Heus, C.; Mance, D.; van Doorn, S.; Houben, K.; PopovČeleketić, D.; Damman, R.; Katrukha, E.A.; Jain, P.; et al. EGFR Dynamics Change during Activation in Native Membranes as Revealed by NMR. Cell 2016, 167, 1241-1251.

30. Roovers, R.C.; Laeremans, T.; Huang, L.; de Taeye, S.; Verkleij, A.J.; Revets, H.; de Haard, H.J.; van Bergen En Henegouwen, P.M.P. Efficient inhibition of EGFR signalling and of tumour growth by antagonistic anti-EGFR Nanobodies. Cancer Immunol. Immun. 2007, 56, 303-317.

31. Rahimi, N. Vascular endothelial growth factor receptors: Molecular mechanisms of activation and therapeutic potentials. Exp. Eye.Res. 2006, 83, 1005-1016.

Behdani, M.; Zeinali, S.; Khanahmad, H.; Karimipour, M.; Asadzadeh, N.; Azadmanesh, K.; Khabiri, A.; Schoonooghe, S.; Habibi Anbouhi, M.; Hassanzadeh-Ghassabeh, G.; et al. Generation and characterization of a functional Nanobody against the vascular endothelial growth factor receptor-2; angiogenesis cell receptor. Mol. Immunol. 2012, 50, 35-41.

33. Gampel, A.; Moss, L.; Jones, M.C.; Brunton, V.; Norman, J.C.; Mellor, H. VEGF regulates the mobilization of VEGFR2/KDR from an intracellular endothelial storage compartment. Blood 2006, 108, 2624-2631.

34. Jopling, H.M.; Howell, G.J.; Gamper, N.; Ponnambalam, S. The VEGFR2 receptor tyrosine kinase undergoes constitutive endosome-to-plasma membrane recycling. Biochem. Biophys. Res. Commun. 2011, 410, 70-176.

35. Chen, Y.Y.; Syed, A.M.; MacMillan, P.; Rocheleau, J. v.; Chan, W.C.W. Flow Rate Affects Nanoparticle Uptake into Endothelial Cells. Adv. Mater. 2020, 32, 1-7.

36. Martínez-Jothar, L.; Barendrecht, A.D.; de Graaff, A.M.; Oliveira, S.; van Nostrum, C.F.; Schiffelers, R.M.; Hennink, W.E.; Fens, M.H.A.M. Endothelial Cell Targeting by cRGDFunctionalized Polymeric Nanoparticles under Static and Flow Conditions. Nanomaterials 2020, 10, 1353-1372.

37. Mashayekhi, V.; Op 'T Hoog, C.; Oliveira, S. Vascular targeted photodynamic therapy: A review of the efforts towards molecular targeting of tumor vasculature. J. Porphyr. Phthalocya. 2019, 23, 1229-1240.

38. Frochot, C.; Stasio, B. di; Vanderesse, R.; Belgy, M.J.; Dodeller, M.; Guillemin, F.; Viriot, M.L.; Barberi-Heyob, M. Interest of RGD-containing linear or cyclic peptide targeted tetraphenylchlorin as novel photosensitizers for selective photodynamic activity. Bioorg. Chem. 2007, 35, 205-220.

39. Tirand, L.; Frochot, C.; Vanderesse, R.; Thomas, N.; Trinquet, E.; Pinel, S.; Viriot, M.L.; Guillemin, F.; Barberi-Heyob, M. A peptide competing with VEGF165 binding on neuropilin-1 mediates targeting of a chlorin-type photosensitizer and potentiates its photodynamic activity in human endothelial cells. J. Controlled Release 2006, 111, 153164.

40. Driehuis, E.; Spelier, S.; Beltrán Hernández, I.; de Bree, R.; M. Willems, S.; Clevers, H.; Oliveira, S. Patient-Derived Head and Neck Cancer Organoids Recapitulate EGFR Expression Levels of Respective Tissues and Are Responsive to EGFR-Targeted Photodynamic Therapy. J. Clin. Med. 2019, 8, 1-17. 
41. Li, K.; Zhang, W.; Fang, H.; Xie, W.; Liu, J.; Zheng, M.; Wang, X.; Wang, W.; Tan, W.; Cheng, H. Superoxide flashes reveal novel properties of mitochondrial reactive oxygen species excitability in cardiomyocytes. Biophys. J. 2012, 102, 1011-1021.

42. Chen, B.; Pogue, B.W.; Hoopes, P.J.; Hasan, T. Combining vascular and cellular targeting regimens enhances the efficacy of photodynamic therapy. Int. J. Radiat. Oncol. Biol. Phys. $\mathbf{2 0 0 5}, 61,1216-1226$. 


\section{Supplementary Data}

\section{Figure S1}

(a)

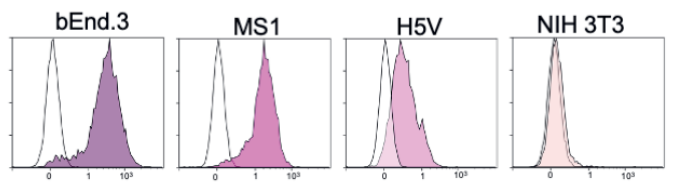

(b)

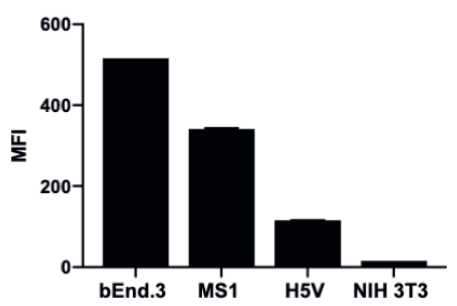

Figure S1. VEGFR2 expression in the murine cell line measured by flow cytometry. (a) Fluorescence of VEGFR2 detected by anti-VEGFR2-PE antibody correlates with the level of VEGFR2 expression: bEnd.3> MS1> H5V > NIH 3T3. The murine cell lines were incubated with the antibody for $1 \mathrm{~h}$ at $37{ }^{\circ} \mathrm{C}$ followed by trypsinization and FACS analysis. (b) Mean fluorescent intensity (MFI) obtained from flow cytometry. Data shown as mean \pm SD.

\section{Figure S2}
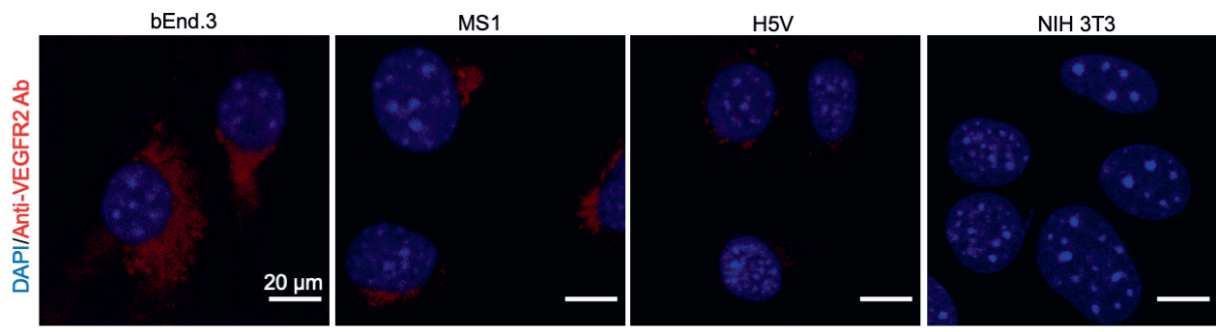

Figure S2. Microscopic images of VEGFR2 expression in the murine cell line. Fluorescence of VEGFR2 detected by anti-VEGFR2 antibody showed perinuclear VEGFR2 staining with varying intensities correlating with the level of VEGFR2, and no signal was observed in the NIH 3T3 cells. 


\section{Figure S3}

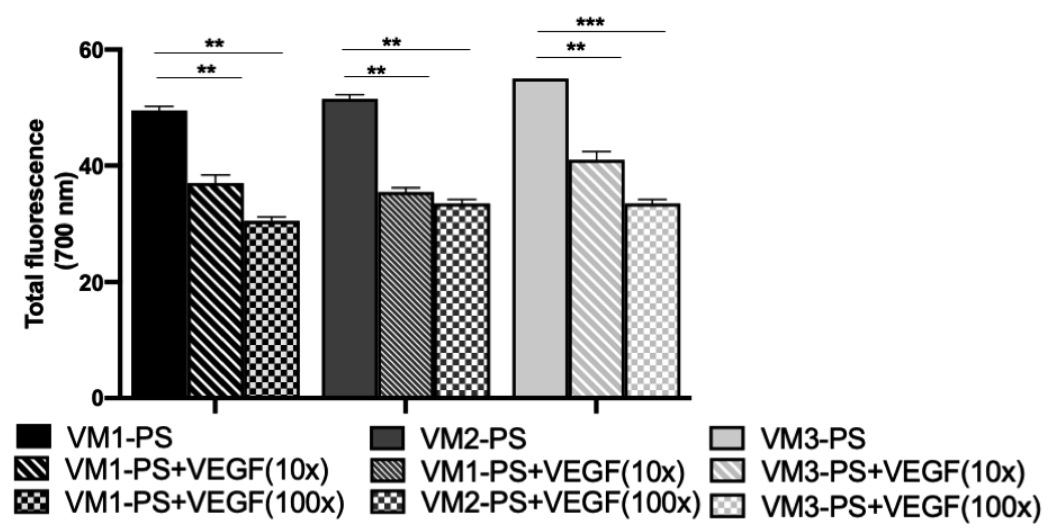

Figure S3. In vitro competition experiment of the NB-PS conjugates tested on purified protein in the presence or absence of 10x and 100x excess of VEGF. NB-PS conjugates alone (final concentration $25 \mathrm{nM}$ ) or in combination with VEGF (final concentrations $250 \mathrm{nM}$ or $2.5 \mu \mathrm{M}$ ) were added to the mVEGFR2 protein and incubated for $2 \mathrm{~h}$ at RT. After 3 times washing with PBS, total fluorescence intensity of the bound NB-PS conjugates was detected by an Odyssey infrared scanner at $700 \mathrm{~nm}$ $(* * \mathrm{p}<0.01 ; * * * \mathrm{p}<0.001 ; \mathrm{t}$ test $)$.

\section{Figure S4}

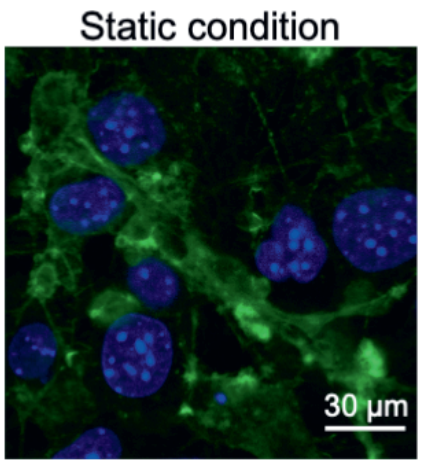

\section{Flow condition}

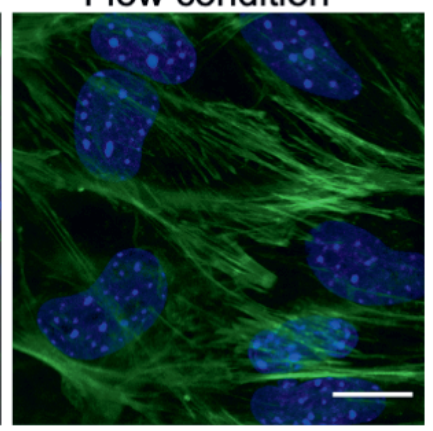

Figure S4. Comparison of $\mathrm{F}$ actin staining in non-treated bEnd.3 cells cultured under static or flow conditions. The $\mathrm{F}$ actin cytoskeleton staining shows a clear difference between the two conditions. A clear alignment of actin fibers is observed when cells were kept under flow. 


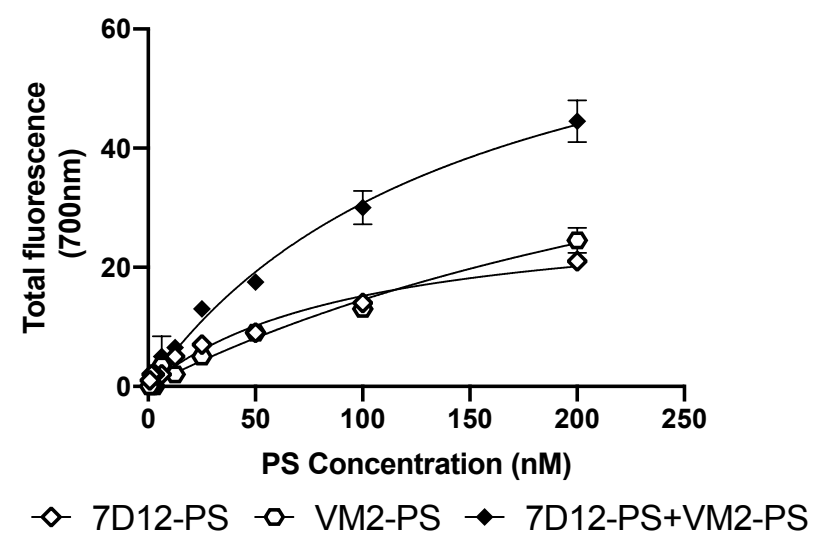

Figure S5. Association of the VEGFR2 and EGFR targeted nanobodies, separately and in combination, with cells of MS1/OSC co-cultures. After $1 \mathrm{~h}$ incubation of cells with the conjugates at $37^{\circ} \mathrm{C}$, total fluorescence intensity of the associated NB-PS conjugates was detected by an Odyssey infrared scanner at $700 \mathrm{~nm}$. 


\section{Chapter 7}

\section{General Discussion}

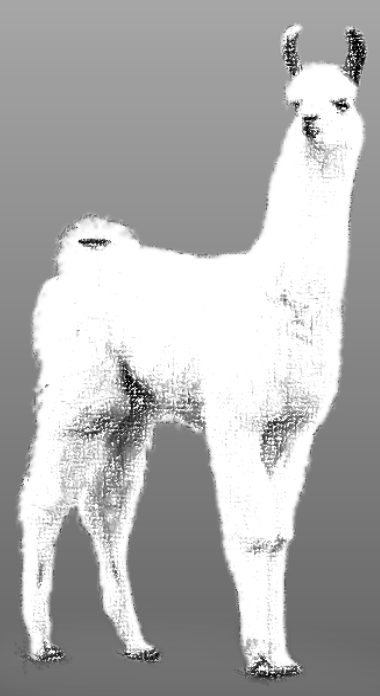




\section{Targeted PDT for cancer treatment}

Targeted PDT is gaining more and more attention in the field of oncology, as it improves both specificity and efficiency of nontargeted (conventional) PDT and overcomes some of its current limitations, namely photosensitivity and damage to the surrounding tissues. Importantly, recently more hydrophilic PSs have been developed which on their own do not stick to the cells and are not able to cross cellular membranes, and have accelerated the development of PDT targeted to tumor-associated markers [1]. Nanobodytargeted PDT was recently developed in order to improve the treatment efficacy of antibody-targeted PDT and further reduce side effects [2]. In this approach, PS molecules are specifically and selectively associated with tumor cells by means of nanobodies. Using EGFR targeted nanobodies, light could be applied shortly after the intravenous administration of the nanobody-photosensitizer conjugates, leading to specific tumor damage in a mouse model of oral cancer [3]. Moreover, it has been shown that nanobodies distribute more homogenously in the tumor tissue than antibodies, leading to more extensive tumor necrosis [3].

In order to further explore the potential of this approach for cell-targeted PDT, in

this thesis we used anti-US28 and anti-Met nanobodies which are (over)expressed on tumor cells. The expression of US28 has been detected in multiple tumors including gliomas, colorectal cancer and prostate cancer [4,5]. Of note, the expression of US28 as a foreign viral encoded target in tumors and not in the surrounding healthy tissues, makes it an ideal target for therapy. Overexpression of Met has been reported in many types of solid tumors such as lung, colorectal, breast, gastric cancers [6,7]. Overall, we showed that nanobodytargeted PDT effectively and specifically induced cell death in cancer cells expressing US28 or Met, as discussed in detail in Chapter 2 and Chapter 3.

Several parameters should be taken into consideration when targeted PDT is applied. The important prerequisite for successful targeted PDT is obtaining higher accumulation of the PS molecules on the tumor tissue compared to the normal cells, after the administration of the conjugate. This can be achieved by targeting of the receptors that preferably are only or more abundantly present on the tumor cell surface [1]. There are some targets such as EGFR and Met which are not only overexpressed on the surface of cancer cells but also are expressed on healthy cells of many tissues. Due to the fact that light is applied locally on the tumor site after the administration of the conjugates, the 
effects on the normal tissues will be minimized. As a consequence, targeted PDT has two levels of specificity: the specific binding to the target molecule and the specific application of the PS activating light. The structure and internalization kinetics of the receptors are also important to consider for targeted therapy. The targeted receptor should have enough extracellular domain surface area for the conjugate to be able to interact. Moreover, the internalization rate of the receptor could influence the overall cytotoxicity, as it could influence the amount of PS delivered intracellularly, thus this should be investigated carefully for each receptor. This is because receptor internalization delivers PS to the cytoplasm and intracellular organelles, which can result in more effective PDT response [8]. In the context of nanobody-targeted PDT, the binding of biparatopic nanobodies causes receptor clustering and internalization, which would favor intracellular accumulation of the conjugate, compared to monovalent nanobodies which are more slowly internalized through fluid phase uptake [2]. However, their increase in size can affect tumor distribution, as in vitro imaging of spheroids demonstrated a reduction in penetration with increasing the size of nanobodies [9].

Another important determinant of a successful PDT is the amount of PS delivered to the tumor site at the time of illumination. In principle, the more PS molecules are present at the tumor site, the more reactive species are produced. There are different methods for the conjugation of PSs to the carrier (being antibody or nanobody). With conjugation of the PS to multiple lysines via random conjugation, potentially a conjugation degree more than 1 can be achieved. However, conjugation to multiple lysines cannot be controlled easily and can also significantly affect the binding properties of the nanobody (as observed for the US28 nanobody). The problem of affinity loss can be solved by following the site-directed conjugation. Free cysteine-maleimide reaction, sortase mediated protein ligation, and click chemistry are most commonly used for the site-directed labeling of antibodies or nanobodies [10-14]. The side-directed conjugation will result in a homogenous product which could be characterized more easily. It is important to note that although compounds labeled via random conjugation are used in the clinic, this approach should be applied with caution for small molecules such as nanobodies (compared to antibodies) as the binding affinity could be compromised.

It should be taken into consideration that a maximal degree of conjugation of 1 is in general achieved by site-directed conjugation. Therefore, other modifications such as the 
fusion of peptide tail with multiple conjugation site such as cysteines or click chemistry residues might be a way to increase the number of PS molecules per therapeutic agent.

Besides the specific and preferably high levels of PS accumulation at the tumor site compared to the normal tissue, a good distribution of the PS is preferred, ideally reaching each single cancer cells to get an effective PDT response. Nevertheless, also immune and vascular effects are expected after PDT [15] which altogether contribute to antitumor effects. Using intravital microscopy, we revealed that beside the specific cell cytotoxicity, vascular effects are also induced after EGFR-targeted nanobody-PS mediated PDT (Chapter 4). Moreover, we showed that monovalent nanobody is slightly better than biparatopic nanobody in inducing tumor necrosis, likely due to smaller size and better distribution in the tumor.

\section{Combination therapy}

Single receptor targeted therapies are not always capable of complete tumor eradication, mainly due to the heterogenous composition of the tumor cells and the low/moderate level of target receptor expression. Dual or multiple targeting can potentially overcome this problems and improve the therapeutic efficacy of targeted PDT [16]. Ideal targets are the ones that $a$. are (over)expressed on the surface of tumor cells and not on the surrounding normal tissues, $b$. have enough extracellular domain for interaction with the therapeutic agents and, $c$. are found in a high percentage of patients. Intratumoral heterogeneity in target expression is known in most of the tumors especially within the bulk tumors in which cell subpopulations exist with heterogenous expression of cell surface receptors [17]. In the context of targeted PDT, due to the small size of nanobodies compared to the antibodies, a combination of nanobodies targeting several non-overlapping epitopes might improve PS accumulation in the heterogenous tumors and, as a result, the photodynamic destruction of tumor cells. Another approach to apply combination therapy is the targeting of tumor vasculature to impair blood flow and consequently blocking the nutrients and oxygen supply to the tumor cells. This in combination with cell targeting can improve the anti-tumor effects of PDT. Applying light shortly after the PS administration causes damage to the tumor-associated vessels. This approach has been named vascular targeted PDT (VTP) [18]. In order to increase the association of the PS specifically with the tumor vasculature, targeting of certain markers on the endothelial cells has been 
investigated. This strategy is described as molecular targeted VTP [19] and has been explored for proteins such as extra domains of fibronectin (A, B), integrin $\alpha v \beta 3$, neuropilin-1, among others (Chapter 5).

Even though nanobody-targeted PDT was shown to induce permanent vascular damage in areas of EGFR-expressing tumor cells (Chapter 4), we hypothesized that simultaneous targeting of cancer cells and tumor-associated vasculature would result in more effective nanobody-targeted PDT in vivo. In Chapter 6, we showed that the combination of cancer and endothelial cell targeting results in more potent in vitro PDT efficacy, compared to the individual treatments. The fact that anti-VEGFR2 nanobodies exclusively bind to the mouse VEGFR2 will enable dedicated studies on the effects of treatment on tumor vasculature. In vivo studies will be conducted to first explore the association of fluorescently-labeled nanobodies with the tumor vasculature using intravital microscopy and, secondly, to assess the efficacy of the conjugates in an orthotopic mouse model with human tumor, either targeting cancer or vasculature individually or both. Of note, as some tumors such as pancreatic cancer are hypovascularized, the model for the combined therapy should be selected with caution.

Since the nanobodies targeting endothelial cells are exposed to the blood flow, association to the target with high association rates (affinity) is very important. Association of the VEGFR2 targeted nanobodies with endothelial cells was verified using the ibidi pump system. Our first in vivo studies will provide crucial details on such association. Yet, in case these nanobodies are too rapidly cleared from the bloodstream, some strategies can be applied to improve half-life and retention time of the nanobodies at the tumor vasculature. For instance, the fusion of serum albumin or albumin binding domain to the nanobody can increase the half-life of the conjugates [20]. Alternatively, decoration of nanoparticles' surface with the anti-VEGFR2 nanobodies is one of the strategies which can improve affinity by avidity and possibly also the PS pharmacokinetics and the retention time of the PS [20]. However, such strategies would have to be tested and fine-tuned, as such modifications affect the size of the conjugate, its tissue distribution, and prolong its half-life. This could lead to longer waiting time between administration of the conjugates and light application.

Interestingly, the fact that the anti-VEGFR2 nanobodies could inhibit VEGFinduced proliferation opens up a possibility to explore the application of these nanobodies 
as anti-angiogenic agents. Our preliminary results showed that one of the anti-VEGFR2 nanobodies (VM2) could inhibit tube formation, which is the most common in vitro test for angiogenesis inhibition. Further in vitro and in vivo experiments are needed to explore the potential of these nanobodies as anti-angiogenic agents.

\section{In vitro and preclinical models for targeted PDT}

A great number of preclinical studies are performed using new compounds before a drug reaches the clinic. Two-dimensional platforms in which flat monolayer cells are cultured are still the most commonly used for the research of cell-based assays as they are easy, convenient and cost-effective. The major drawback is that this system does not mimic the tumor microenvironment or stroma. For instance, morphological characteristics, proliferation and differentiation potentials, cell-cell interactions, and composition of stroma fluid (low pH, proteases, etc) are not well recapitulated by 2D cultures. Such concerns inspired the emergence of $3 \mathrm{D}$ cell culture systems. Over the years, a variety of in vitro platforms have been developed to achieve 3D cell culture systems [21]. One of the systems which was described in Chapter 2 is growing spheroids in Ultra-low attachment plates. Recently, these plates have been used extensively for the development of monoculture, coculture and triple-cultures [22-24]. More complex systems such as in vitro organotypic cultures have been established in order to mimic the tumor microenvironment $[25,26]$. Organoid models which are three dimensional self-organizing structures also have been explored recently to screen the in vitro efficacy of therapeutics. They can be established from patient-derived tumor cells, such as surgical resections or tumor biopsies, allowing the in vitro expansion of patient-derived tumors [27]. Nanobody-targeted PDT in a head and neck squamous cell carcinoma-organoid model showed that expression of EGFR in the patient-derived organoids is in the range of HeLa cells or only slightly higher [28], likely in the range of 35,000-50,000 receptors per cell. Nevertheless, tumor organoids were more sensitive to PDT than their corresponding wild-type counterparts (with lower EGFR expression), indicating that with appropriate dosing, this therapy is effective and is also expected to leave the normal cells surrounding the tumor unaffected [28]. The major drawback of in vitro studies is their failure to capture the inherent complexity of organ systems, including the role of the immune system. Conducting in vivo studies using animal models addresses many of the shortcomings of in vitro studies. The problem of 
translatability remains, which originates from the considerable physiological differences between humans and animals that impact pharmacokinetics of tested compounds. Nevertheless, the preclinical evidence of therapeutic efficacy is necessary before moving into clinical trials. The appropriateness of animal models to evaluate the therapeutic efficacy of agents is a serious issue, as the high failure rate of preclinical compounds in clinical trials clearly demonstrates the limitations of existing preclinical models. Subcutaneous syngeneic mouse tumors with intact immune systems or growing human xenografts in athymic nude mice or severe combined immunodeficient mice (B- and T-cell deficient), among other models, are often used to study PDT effects in vivo. The latter provide the opportunity to study human tumor biology. It is important to note that subcutaneous tumors differ in the rate of growth, degree of vascularization, amount of tumor stroma and potential formation of spontaneous metastasis [29]. Moreover, subcutaneous tumors in PDT research do not entirely replicate the normal biology of human tumors, as they are not situated in their organ of origin (ectopic versus orthotopic). Therefore, alternatively, tumor cells have been injected into the tissue of the organ of origin in mice. However, generally in small animals, orthotopic tumors may be more difficult to handle. It also makes applying the light to the tumor site more difficult [29]. Importantly, it should be taken into consideration that the anti-tumor effects observed after PDT with immunocompromised mice might be different than in mice with a complete immune system, as the full anti-tumor immune reactions after PDT do not occur in the former and have been shown to be important for the long term anti-tumor effects.

\section{Nanobody-targeted PDT in perspective}

Several in vitro and in vivo studies have shown the potential of nanobody-targeted PDT for cancer treatment. Recently we obtained insights with unprecedented detail about the mechanism of nanobody-targeted PDT. In addition, it has been shown that an immune response is triggered after nanobody-targeted PDT in vitro [30]. Still, more preclinical studies are needed in order to explore the full potential of this promising approach, paving the way for its further clinical development. Currently, a number of nanobodies are under clinical investigation for the imaging of a wide spectrum of human diseases including inflammation, breast cancer, and brain tumors [31]. Furthermore, the hydrophilic PS IRDye700DX, is currently investigated in a Phase III trial, where the mAb Cetuximab 
targeting EGFR is conjugated to the PS IRDye700DX for the PIT of patients with recurrent head and neck cancers (NCT02422979). The outcome of such clinical trials will certainly influence further evaluation of nanobody-targeted PDT approach in the clinic.

\section{References}

1. Solban, N.; Rizvi, I.; Hasan, T. Targeted photodynamic therapy. Lasers Surg. Med. 2006, 38, 522-531.

2. Heukers, R.; van Bergen en Henegouwen, P.M.P.; Oliveira, S. Nanobody-photosensitizer conjugates for targeted photodynamic therapy. Nanomed. Nanotechnol. 2014, 10, 14411451.

3. van Driel, P.B.A.A.; Boonstra, M.C.; Slooter, M.D.; Heukers, R.; Stammes, M.A.; Snoeks, T.J.A.; de Bruijn, H.S.; van Diest, P.J.; Vahrmeijer, A.L.; van Bergen en Henegouwen, P.M.P.; et al. EGFR targeted nanobody-photosensitizer conjugates for photodynamic therapy in a pre-clinical model of head and neck cancer. Journal Controlled Release 2016, $229,93-105$

4. Soroceanu, L.; Matlaf, L.; Bezrookove, V.; Harkins, L.; Martinez, R.; Greene, M.; Soteropoulos, P.; Cobbs, C.S. Human cytomegalovirus US28 found in glioblastoma promotes an invasive and angiogenic phenotype. Cancer Res. 2011, 71, 6643-6653.

5. Samanta, M.; Harkins, L.; Klemm, K.; Britt, W.J.; Cobbs, C.S. High prevalence of human cytomegalovirus in prostatic intraepithelial neoplasia and prostatic carcinoma. J. Urol. $\mathbf{2 0 0 3}$ 170, 998-1002.

6. Peruzzi, B.; Bottaro, D.P. Targeting the c-Met signaling pathway in cancer. Clin. Cancer Res. 2006, 12, 3657-3660.

7. Birchmeier, C.; Birchmeier, W.; Gherardi, E.; vande Woude, G.F. Met, metastasis, motility and more. Nat.Rev. Mol. 2003, 4, 915-925.

8. Vrouenraets, M.B.; Visser, G.W.M.; Stigter, M.; Oppelaar, H.; Snow, G.B.; van Dongen, G.A.M.S. Targeting of Aluminum (III) Phthalocyanine Tetrasulfonate by Use of Internalizing Monoclonal Antibodies: Improved Efficacy in Photodynamic Therapy. Cancer Res. 2001, 61, 1970-1975.

9. Beltrán Hernández, I.; Rompen, R.; Rossin, R.; Xenaki, K.T.; Katrukha, E.A.; Nicolay, K.; van Bergen en Henegouwen, P.; Grüll, H.; Oliveira, S. Imaging of Tumor Spheroids, DualIsotope SPECT, and Autoradiographic Analysis to Assess the Tumor Uptake and Distribution of Different Nanobodies. Mol. Imaging Biol. 2019, 21, 1079-1088.

10. Kim, Y.; Ho, S.O.; Gassman, N.R.; Korlann, Y.; Landorf, E. v.; Collart, F.R.; Weiss, S. Efficient Site-Specific Labeling of Proteins via Cysteines. Bioconjug. Chem. 2008, 19, 786791.

11. Gonzalez-Sapienza, G.; Rossotti, M.A.; Tabares-da Rosa, S. Single-Domain Antibodies As Versatile Affinity Reagents for Analytical and Diagnostic Applications. Frontiers in Immunology 2017, 8, 1-12.

12. Peplow, M. Click chemistry targets antibody-drug conjugates for the clinic. Nature biotechnol. 2019, 37, 835-837.

13. Wübbeke, L.F. Nanobody-photosensitizer conjugates for the treatment of epithelial ovarian cancer through targeted photodynamic therapy. In Proceedings of the Student Research Conference; 2016; pp. 1-4.

14. Lee, S.; Koo, H.; Na, J.H.; Han, S.J.; Min, H.S.; Lee, S.J.; Kim, S.H.; Yun, S.H.; Jeong, S.Y.; Kwon, I.C.; et al. Chemical Tumor-Targeting of Nanoparticles Based on Metabolic Glycoengineering and Click Chemistry. ACS Nano 2014, 8, 2048-2063.

15. Reginato, E. Immune response after photodynamic therapy increases anti-cancer and antibacterial effects. World J. Immunol. 2014, 4, 1. 
16. Bano, S.; Obaid, G.; Swain, J.W.R.; Yamada, M.; Pogue, B.W.; Wang, K.; Hasan, T. NIR Photodynamic Destruction of PDAC and HNSCC Nodules Using Triple-Receptor-Targeted Photoimmuno-Nanoconjugates: Targeting Heterogeneity in Cancer. J. Clin. Med. 2020, 9, 2390.

17. Pribluda, A.; de la Cruz, C.C.; Jackson, E.L. Intratumoral Heterogeneity: From Diversity Comes Resistance. Clin. Cancer Res. 2015, 21, 2916-2923.

18. Mazor, O.; Brandis, A.; Plaks, V.; Neumark, E.; Rosenbach-Belkin, V.; Salomon, Y.; Scherz, A. WST11, A Novel Water-Soluble Bacteriochlorophyll Derivative; Cellular uptake, Pharmacokinetics, Biodistribution, and Vascular Targeted Photodynamic Activity Against Melanoma tumors. Photochemistry and Photobiology 2004, 81, 342-351.

19. Mashayekhi, V.; Op 'T Hoog, C.; Oliveira, S. Vascular targeted photodynamic therapy: A review of the efforts towards molecular targeting of tumor vasculature. J. Porphyr. Phthalocya. 2019, 23, 1229-1240.

20. Hu, Y.; Liu, C.; Muyldermans, S. Nanobody-Based Delivery Systems for Diagnosis and Targeted Tumor Therapy. Front. Immunol. 2017, 8, 1-17.

21. Chaicharoenaudomrung, N.; Kunhorm, P.; Noisa, P. Three-dimensional cell culture systems as an in vitro platform for cancer and stem cell modeling. World J. Stem Cells 2019, 11, $1065-1083$.

22. Amann, A.; Zwierzina, M.; Gamerith, G.; Bitsche, M.; Huber, J.M.; Vogel, G.F.; Blumer, M.; Koeck, S.; Pechriggl, E.J.; Kelm, J.M.; et al. Development of an innovative 3D cell culture system to study tumour - Stroma interactions in non-small cell lung cancer cells. PLoS ONE 2014, 9, e92511.

23. Amann, A.; Zwierzina, M.; Koeck, S.; Gamerith, G.; Pechriggl, E.; Huber, J.M.; Lorenz, E.; Kelm, J.M.; Hilbe, W.; Zwierzina, H.; et al. Development of a 3D angiogenesis model to study tumour - endothelial cell interactions and the effects of anti-angiogenic drugs. Sci. Rep. 2017, 7, 2963.

24. Lazzari, G.; Nicolas, V.; Matsusaki, M.; Akashi, M.; Couvreur, P.; Mura, S. Multicellular spheroid based on a triple co-culture: A novel 3D model to mimic pancreatic tumor complexity. Acta Biomater. 2018, 78, 296-307.

25. Coleman, S.J. Pancreatic cancer organotypics: High throughput, preclinical models for pharmacological agent evaluation. World J. Gastroenterol. 2014, 20, 8471.

26. Froeling, F.E.M.; Mirza, T.A.; Feakins, R.M.; Seedhar, A.; Elia, G.; Hart, I.R.; Kocher, H.M. Organotypic Culture Model of Pancreatic Cancer Demonstrates that Stromal Cells Modulate E-Cadherin, $\beta$-Catenin, and Ezrin Expression in Tumor Cells. Am. J. Pathol. 2009, 175, 636-648.

27. Kretzschmar, K.; Clevers, H. Organoids: Modeling Development and the Stem Cell Niche in a Dish. Develop. Cell 2016, 38, 590-600.

28. Driehuis, E.; Spelier, S.; Beltrán Hernández, I.; de Bree, R.; M. Willems, S.; Clevers, H.; Oliveira, S. Patient-Derived Head and Neck Cancer Organoids Recapitulate EGFR Expression Levels of Respective Tissues and Are Responsive to EGFR-Targeted Photodynamic Therapy. J. Clin. Med. 2019, 8, 1-17.

29. Silva, Z.S.; Bussadori, S.K.; Fernandes, K.P.S.; Huang, Y.-Y.; Hamblin, M.R. Animal models for photodynamic therapy (PDT). Biosci. Rep. 2015, 35, 1-14.

30. Beltrán Hernández, I.; Yu, Y.; Ossendorp, F.; Korbelik, M.; Oliveira, S. Preclinical and Clinical Evidence of Immune Responses Triggered in Oncologic Photodynamic Therapy: Clinical Recommendations. J. Clin. Med. 2020,9, 333.

31. Jovčevska, I.; Muyldermans, S. The Therapeutic Potential of Nanobodies. Bio Drugs 2020 , $34,11-26$. 


\section{Appendices}

English summary

Nederlandse samenvatting

List of publications

Curriculum Vitae

Acknowledgments

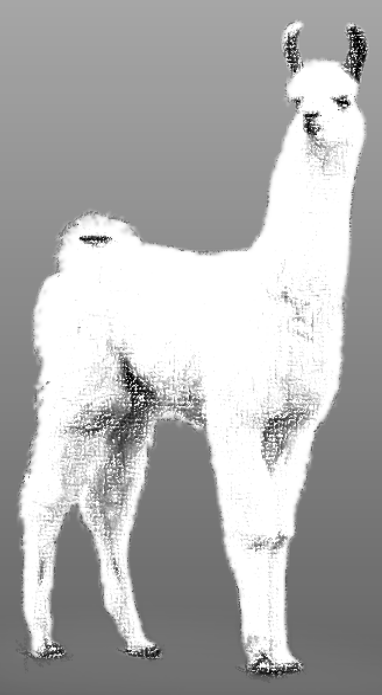




\section{Appendix}

English summary 
Photodynamic therapy (PDT) is a minimally invasive approach for cancer treatment. In this approach three essential elements are needed to induce local cytotoxicity: a light-activatable photosensitizer (PS), light of a specific wavelength, and oxygen molecules. The activated PS can transfer energy to oxygen and subsequently form cytotoxic reactive oxygen species (ROS), among other reactive molecular species which can destroy tumor cells, damage tumor vasculature, and also induce immune responses. Nanobody-targeted PDT was recently developed in order to improve treatment efficacy and further reduce side effects associated with conventional PDT. With the promising results obtained previously, in this thesis first we explored nanobody-targeted PDT with molecular targets other than EGFR; the second aim was to understand the vascular effects induced by nanobody-targeted PDT; and the third aim was to investigate the combination of nanobody targeting cancer and endothelial cells for more effective PDT.

In Chapter 2, the potential of G protein-coupled receptors (GPCRs) as target for nanobody-targeted PDT was presented, by using the HCMV-encoded chemokine receptor US28.

US28, one of the four HCMV-encoded viral GPCRs, has been detected in multiple tumors of HCMV-positive patients, including gliomas. Nanobody-targeted PDT specifically induced cell death in the US28 expressing glioblastoma cells in 2D and 3D cell models, upon illumination with near-infrared light. In parallel, we also described the characterization of a nanobody-PS conjugate specific for the hepatocyte growth factor receptor (HGFR), also known as c-Met or Met, as an alternative target (Chapter 3). Overexpression of Met has been reported in large number of carcinomas, sarcomas, haematopoietic malignancies and other neoplasms. We showed that the nanobody-PS conjugate, induced cell death specifically and effectively in Met overexpressing cancer cells.

In Chapter 4, further insights were obtained into nanobody-targeted PDT mechanism of tumor destruction, focusing on vascular effects in more detail. We investigated the biodistribution of two different formats of nanobodies targeting EGFR (7D12 as monovalent and 7D12-9G8 as biparatopic), in vivo using intravital microscopy. Subsequently, cellular responses and vascular effects of EGFR-targeted PDT were investigated. Both nanobody-PS conjugates showed more fluorescence in tumor than in distant normal tissue at 1 or $2 \mathrm{~h}$ after administration. 7D12-9G8-PS showed an intense, 
membrane localized, fluorescence pattern in tumor cells, while 7D12-PS fluorescence was more diffuse. After illumination at $1 \mathrm{~h}$ post injection, distinct changes to the morphology of tumor cells were observed in the GFP fluorescence images recorded in time. Importantly, besides the tumor response after PDT, vascular responses were also observed. The proportional area of tumor that showed either lack of flow or reduced flow $2 \mathrm{~h}$ post PDT was similar for both conjugates when light was applied $1 \mathrm{~h}$ after administration. The results showed that the overall acute response to EGFR-targeted NB-PS mediated PDT is a complex mixture of tumor cell response and vascular effects

We were also interested in exploring targeting of the vasculature specifically with dedicated conjugates. Therefore, we first reviewed studies that have been performed in the last 15 years to deliver PS selectively to the tumor vasculature for more cancer specific PDT (Chapter 5). As efficacy of targeted PDT can be hampered by heterogeneity of target expression and/or moderate/low target expression levels, we explored the possibility of combined targeting of endothelial and cancer cells in vitro. For this, in Chapter 6, we developed nanobodies binding to the mouse VEGFR2, which is overexpressed on tumor vasculature, and combined these with nanobodies specific for the cancer cell target EGFR. The cytotoxicity of these conjugates in monocultures and in co-cultures with cancer cells showed that the anti-VEGFR2 conjugates are specific and potent PDT agents. Nanobodytargeted PDT in co-culture of endothelial and cancer cells showed improved efficacy, when VEGFR2 and EGFR targeting nanobodies were applied simultaneously, compared to the separate treatments.

In conclusion in this thesis we showed that nanobody-targeted PDT can be applied for targeting of different receptors, for which moderate/high expression levels are necessary. Using intravital microscopy, we revealed that beside specific cell cytotoxicity, vascular effects are also induced with this approach. Finally, simultaneous targeting of endothelial and cancer cells improved nanobody-targeted efficacy in vitro, a promising approach to overcome heterogenous or lower levels of target expression. Follow-up studies will aim at the efficacy evaluation of the combined conjugates in mice with human tumors to reveal the full potential of this promising approach. 


\section{Appendix}

Nederlandse samenvatting 
Fotodynamische therapie (PDT) is een weinig invasieve behandeling voor patiënten met kanker. Bij deze behandeling zijn er drie essentiële elementen nodig om lokale cytotoxiciteit te induceren bij de kankercellen: de fotosensibilisator (PS), licht van een bepaalde golflengte en zuurstofmoleculen. De geactiveerde PS kan vervolgens energie overdragen aan zuurstof en hierop volgend cytotoxische reactieve zuurstofcomponenten (ROS) vormen. Daarnaast worden er ook andere reactieve moleculaire componenten gevormd die samen met ROS in staat zijn om tumorcellen te doden, tumorvaten te beschadigen en een immuunrespons te induceren. Op nanobody gebaseerde PDT werd onlangs ontwikkeld om de efficiëntie van de behandeling van kanker te verbeteren en de bijwerkingen van de conventionele PDT verder te verminderen. Gebaseerd op de veelbelovende eerdere resultaten hebben we in dit proefschrift eerst nanobody gebaseerde PDT opties onderzocht met andere moleculaire targets dan EGFR; het tweede doel was om de vasculaire effecten die worden veroorzaakt door nanobody gebaseerde PDT beter te begrijpen; ten slotte was het derde doel om de combinatie van nanobodies gericht op zowel kanker als endotheelcellen te onderzoeken voor een effectievere PDT.

In Hoofdstuk 2 werd het potentieel van G-proteïnegekoppelde receptoren (GPCR's) als doelwit voor nanobody gebaseerde PDT uitgelicht, door ons te richten op de HCMV gecodeerde chemokinereceptor US28. US28, een van de vier HCMV gecodeerde virale GPCR's, is reeds gedetecteerd in meerdere tumoren van HCMV-positieve patiënten, waaronder gliomen. Nanobody gebaseerde PDT induceerde selectief cytotoxiciteit in de US28 positieve glioblastomacellen in 2D- en 3D-celmodellen na belichting met nabijinfrarood licht. Parallel hieraan hebben we een nanobody-PS-conjugaat beschreven dat specifiek is voor de hepatocyte groeifactor receptor (HGFR), ook wel bekend als c-Met of Met, als alternatief doelwit voor PDT (Hoofdstuk 3). Overexpressie van Met is geobserveerd bij een groot aantal carcinomen, sarcomen, hematopoëtische maligniteiten en andere neoplasmata. We toonden aan dat het nanobody-PS-conjugaat selectief en effectief cytotoxiciteit induceerde in kankercellen die overexpressie van Met vertoonden.

In Hoofdstuk 4 werden meer inzichten verkregen in de mechanismen waarop nanobody gebaseerde PDT leidt tot cytotoxiciteit in tumoren, waarbij de nadruk meer lag op het onderzoeken van de vasculaire effecten. We onderzochten de biodistributie van twee verschillende vormen van EGFR gerichte nanobodies (7D12 als monovalent en 7D12-9G8 als biparatoop) in vivo met behulp van intravitale microscopie. Vervolgens werden 
cellulaire responsen en vasculaire effecten van de op EGFR gerichte PDT onderzocht. Beide nanobody-PS-conjugaten vertoonden meer fluorescentie in de tumor in vergelijking met normaal weefsel op 1 en 2 uur na toediening. 7D12-9G8-PS vertoonde een intens, membraan-gelokaliseerd fluorescentiepatroon in tumorcellen, terwijl 7D12-PSfluorescentie diffuser was. 1 uur na injectie vond belichting van de tumorcellen plaats waarop vervolgens duidelijke veranderingen in de morfologie van tumorcellen over de loop van tijd werden waargenomen aan de hand van de GFP-fluorescentiebeelden. Naast de tumorrespons bij deze behandeling werden ook vasculaire responsen waargenomen. Het deel van de tumor dat ofwel een gebrek ofwel een vermindering aan perfusie vertoonde was 2 uur na PDT en 1 uur na belichting vergelijkbaar voor beide conjugaten. De resultaten toonden aan dat de complete acute respons van op EGFR-gerichte nanobody PDT een complexe mix is van tumorcelresponsen en vasculaire effecten.

Ook waren we geïnteresseerd in het onderzoeken van PDT specifiek gericht op het vaatstelsel van de tumoren met behulp van speciale conjugaten. Daarom hebben we eerst studies beschreven die in de afgelopen 15 jaar zijn uitgevoerd om PS selectief te richt op de bloedvaten van tumoren voor kanker specifiekere PDT (Hoofdstuk 5). Omdat de werkzaamheid van PDT belemmerd kan worden door heterogeniteit en lage expressie van het doeleiwit, hebben we het potentieel onderzocht van een PDT die gericht is op zowel doeleiwitten van endotheel- en kankercellen in vitro. Hiervoor hebben we in Hoofdstuk 6 nanobodies ontwikkeld die binden aan het doeleiwit VEGFR2, dat tot overexpressie wordt gebracht in endotheelcellen in de tumor. Deze hebben we vervolgens gecombineerd met nanobodies die specifiek zijn voor het doeleiwit EGFR in kankercellen. De cytotoxiciteit van deze conjugaten in monoculturen en in co-culturen met kankercellen toonde aan dat de anti VEGFR2-conjugaten specifieke en sterke PDT middelen zijn. Op nanobody gebaseerde PDT vertoonde een verbeterde werkzaamheid in co-culturen van endotheel- en kankercellen wanneer de op VEGFR2- en EGFR gerichte nanobodies gelijktijdig werden toegediend in vergelijking met de afzonderlijke behandelingen.

In dit proefschrift hebben we laten zien dat nanobody gebaseerde PDT kan worden toegepast voor verschillende receptoren, waarbij matige of hoge expressieniveaus nodig zijn. Met behulp van intravitale microscopie hebben we onthuld dat naast specifieke cytotoxiciteit van tumorcellen ook vasculaire effecten kunnen worden geïnduceerd met deze behandeling. Ten slotte verbeterden de effecten van nanobody gebaseerde PDT door 
het richten van de therapie op doeleiwitten van zowel endotheel- als kankercellen in vitro. Dit is een veelbelovende benadering om heterogene of lage niveaus van doeleiwitten te ondervangen. Vervolgstudies zullen gericht moeten worden op de evaluatie van de werkzaamheid van de gecombineerde conjugaten bij muizen met menselijke tumoren om het complete potentieel van deze veelbelovende therapie te onthullen. 


\section{Appendix}

\section{List of Publications}




\section{List of PhD publications}

1. Demian van Straten, Vida Mashayekhi, Henriette S. de Bruijn, Sabrina Oliveira and Dominic J. Robinson. Oncologic Photodynamic Therapy: basic principles, current clinical status and future directions. Cancers. 2017; 9(2):19.

2. Timo W.M. De Groof ${ }^{\dagger}$, Vida Mashayekhi ${ }^{\dagger}$, et al., Martine J. Smit ${ }^{\ddagger}$, and Sabrina

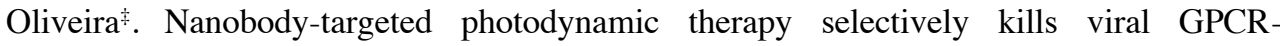
expressing glioblastoma cells. Molecular Pharmaceutics. 2019; 16(7): 3145-3156.

$\dagger, \ddagger$ equal contribution

3. Raimond Heukers, Vida Mashayekhi, et al., Sabrina Oliveira. VHH-photosensitizer conjugates for targeted photodynamic therapy of Met-overexpressing tumor cells. Antibodies. 2019; 8(2): 26.

4. Vida Mashayekhi, Charlotte Op 't Hoog and Sabrina Oliveira. Vascular targeted photodynamic therapy: a review of the efforts towards molecular targeting of tumor vasculature. Journal of Porphyrins and Phtalocyanines. 2019; 23(11): 1229-1240.

5. Henriette S. de Bruijn, Vida Mashayekhi, et al., Sabrina Oliveira. Acute cellular and vascular responses to photodynamic therapy using EGFR-targeted nanobodyphotosensitizer conjugates studied with intravital optical imaging and magnetic resonance imaging. Theranostics. 2020; 10(5): 2436-2452.

6. Vida Mashayekhi, Katerina T. Xenaki, Paul van Bergen en Henegouwen, Sabrina Oliveira. Dual targeting of endothelial and cancer cells potentiates in vitro nanobodytargeted photodynamic therapy. Cancers. 2020; 12(10): 1-18.

\section{Book chapters:}

1. Vida Mashayekhi*, Erik Schooten*, Paul M.P. van Bergen en Henegouwen, Marta M. Kijanka, Sabrina Oliveira, Nanobody-targeted photodynamic therapy: nanobody production and purification. Submitted.

*equal contribution

2. Vida Mashayekhi, Sabrina Oliveira, Conjugation of IRDye photosensitizers or fluorophores to nanobodies. Submitted. 


\section{Other publications}

1. Kamaleddin HME Tehrani, Nora Brüchle, Nicola Wade, Vida Mashayekhi, et al., Nathaniel Martin. Small Molecule Carboxylates Inhibit Metallo- $\beta$-lactamases and Resensitize Carbapenem-Resistant Bacteria to Meropenem. ACS infectious disease. 2020; 6(6): 1366-1371.

2. Kamaleddin HME Tehrani, Haigen Fu, Nora Brüchle, Vida Mashayekhi, et al., Nathaniel Martin. Aminocarboxylic acids related to aspergillomarasmine A (AMA) and ethylenediamine-N,N'-disuccinic acid (EDDS) are strong zinc-binders and inhibitors of the metallo-beta-lactamase NDM-1. Chemical communications. 2020; 56(20): 3047-3049.

3. Michael S M Brouwer, Kamaleddin HME Tehrani, Michel Rapallini, Yvon Geurts, Arie Kant, Frank Harders, Vida Mashayekhi, et al., Kees T Veldman. Novel Carbapenemases FLC-1 and IMI-2 Encoded by an Enterobacter cloacae Complex Isolated from Food Products. Antimicrobial agents and Chemotherapy. 2019; 63(6): e2338-18.

4. Wei Peng, Henriette S de Bruijn, Eric Farrell, Mouldy Sioud, Vida Mashayekhi, Sabrina Oliveira, et al., Dominic J Robinson. Epidermal growth factor receptor (EGFR) density may not be the only determinant for the efficacy of EGFR-targeted photoimmunotherapy in human head and neck cancer cell lines. Lasers in Surgery and Medicine. 2018; 50(5): 513-522.

5. Jibin Guan, Jin Sun, Feilong Sun, Bo Lou, Dong Zhang, Vida Mashayekhi, Negar Sadeghi, Gert Storm, Enrico Mastrobattista, Zhonggui He. Hypoxia-induced tumor cell resistance is overcome by synergistic GAPDH-siRNA and chemotherapy co-delivered by long-circulating and cationic-interior liposomes. Nanoscale. 2017; 9(26): 9190-9201. 


\section{Selected publications before PhD}

1. Vida Mashayekhi, Kamaleddin H.M.E. Tehrani, Jafar Shahraki, Mir-Jamal Hosseini. Mechanistic approach for the toxic effects of perfluorooctanoic acid (PFOA) on isolated rat liver and brain mitochondria. Human and experimental toxicology. 2015; 34: 985-996.

2. Mohammad Reza Eskandari, Vida Mashayekhi, Mohammad Aslani and Mir-Jamal Hosseini. Toxicity of thallium on isolated rat liver mitochondria: The role of oxidative stress and MPT pore opening. Environmental Toxicology. 2015; 30: 232-241.

3. Vida Mashayekhi, Mohammad Reza Eskandari, Farzad Kobarfard, Mir-Jamal Hosseini. Induction of mitochondrial permeability transition (MPT) pore opening and ROS formation as a mechanism for methamphetamine-induced mitochondrial toxicity. NaunynSchmiedeberg's Archives of Pharmacology, 2014; 387: 47-58.

4. Iman Jafarian, Mohammad Reza Eskandari, Vida Mashayekhi, Morteza Ahadpour, MirJamal Hosseini. Toxicity of valproic acid in isolated rat liver mitochondria. Toxicology mechanisms and methods. 2013; 23: 617-623.

5. Vida Mashayekhi, Kamaleddin H.M.E. Tehrani, Parisa Azerang, and Farzad Kobarfard. Synthesis, Antimycobacterial and Anticancer Activity of Novel Indole-Based Thiosemicarbazones. Archives of Pharmacal Research. 2013; 1: 1-13.

6. Vida Mashayekhi, Kamaleddin H.M.E. Tehrani, Salimeh Amidi and Farzad Kobarfard. Synthesis of novel indole hydrazone derivatives and evaluation of their antiplatelet aggregation activity. Chemical and Pharmaceutical Bulletin. 2013; 61: 144-150. 


\section{Appendix}

Curriculum Vitae 


\section{Curriculum Vitae}

Vida Mashayekhi was born in 30 September 1987 in Mianeh, Iran. She studied Pharmacy between 2005 to 2011 in Shahid Beheshti University of Medical Sciences, Tehran, Iran. In the $3^{\text {rd }}$ year national exam (2008), she was ranked $4^{\text {th }}$ among 250 Pharm.D. students in the country. The research topic of her thesis was the development of novel anti-platelet aggregation agents. After she obtained her Pharm.D. degree, she embarked on a new project supervised by Dr. Mohammad Reza Eskandari where she studied the mechanism of mitochondrial toxicity induced by drugs and xenobiotics which led to several publications. After she moved to The Netherlands in 2014, she was hired as a researcher at the Department of Pharmaceutics of Utrecht University under the supervision of Prof. Dr. Enrico Mastrobattista working on the encapsulation of siRNA into liposomal nanocarriers. In May 2016, she started her PhD in the group of Dr. Sabrina Oliveira focusing on the nanobody-targeted photodynamic therapy. During her $\mathrm{PhD}$, she supervised internship projects of two bachelor and four master students. In addition, Vida has also guided three students in their writing assignments. The results of her projects are presented in this dissertation. 


\section{List of courses followed during PhD}

1. Laboratory animal science (Art. 9, 3 ECT)

2. Advanced drug delivery and drug targeting (2 ECT)

3. Light microscopy (1.5 ECT)

4. Introductory biostatistics for researchers (3 ECT)

5. Proteomics and mass spectrometry (1.5 ECT)

6. Introduction to stem cells (1.5 ECT)

7. Advanced immunology (1.5 ECT)

8. UIPS Introductory course (1 ECT)

9. Giving effective oral presentation (0.6 ECT)

\section{List of conferences attended during PhD}

1. International Photodynamic Association (IPA) in Portugal (2017.06)

2. Science for life conference in Utrecht (2018.11)

3. Biopharmacy day in Leiden (2018.11, poster presentation)

4. European Society for Photobiology in Barcelona (2019.08, oral presentation)

5. Science for life conference in Utrecht (2019.11, poster presentation)

6. Nanobody symposium in Bonn (2019.09, poster presentation)

7. PDT-PDD virtual conference (2020.11, oral presentation) 


\section{Appendix}

Acknowledgments 
When I moved to the Netherlands, I started walking in the Uithof, taking pictures, and thinking if I get the chance to start a $\mathrm{PhD}$ at Utrecht University. After a one-year research position in Enrico's lab, I was fortunate to be offered a $\mathrm{PhD}$ position by Sabrina in 2016 and now 4.5 years have passed, and it is time to close this chapter of my life and it feels strange! During my PhD years, I got to know a wonderful group of people with whom I not only shared an interest in science but also built friendships that are dear to my heart.

First of all, I would like to express my deepest gratitude to my first co-promotor, Sabrina. Thank you for giving me the opportunity of doing my $\mathrm{PhD}$ in your group. It was a great pleasure and enjoyable experience to work with you. You are a kind family person, and a scientist with great ideas. You always did your best to make the $\mathrm{PhD}$ life less stressful for me, thank you!

My second co-promotor Paul, I clearly remember the day I sent you an email regarding the possibility of joining your group and you referred me to Sabrina who was about to start her own group. You always had critical comments on the projects which helped me improve my research. Every new year you hosted the group for a dinner which, thanks to your and Eva's hospitality, created unforgettable memories for me.

My promotor Anna, thank you so much for all your supports. Your interventions in different stages of my $\mathrm{PhD}$ were crucial to allow me to conduct my research smoothly even through the difficult situations caused by COVID.

Before I started my $\mathrm{PhD}$, I joined a Friday borrel in DDW to meet some friends. There, I met Wim Hennink and through our conversation he found out that I am interested in Enrico's research projects, but I was too shy to approach him directly to ask for an available position in his group. I never forget what happened next. Wim left our conversation and after 5 minutes came back with Enrico! That meeting led to one year of working in Enrico's lab during which I learned a lot about drug delivery and nano-carrier systems. I sincerely thank both of you.

To our collaborators at Rotterdam Erasmus Medical Center Riette and Dominic. Thank you both for the great collaboration and nice time we had together in Portugal in 2017. I learned a lot from you and look forward to more collaborations in the future. To our collaborators at Vrije Universiteit Amsterdam Martine, Raimond and Timo, it was a great pleasure working with you. 
Sara, Katerina, Sofia, Erik, Sebas and Barbara, I never forget the great times we spent together in the lab and outside. I could rely on your help whenever I needed. Thanks for that! Reinier, we didn't spend too much time together outside the lab but I definitely enjoyed our conversations. Good luck with your future endeavors! Iris, although I very recently had the chance of knowing you a bit, the short chats we had were always pleasant, good luck with the rest of your PhD! Alessia, thank you for being so kind and helpful in the lab.

I would like to thank all of my students Izabela, Apfrida, Brigitte, Mart, Charlotte, Sara and Lola for their hard work during the last 4 years. You all did a good job and I wish you all the best. Bart, Phebe, Illya, and Eugene, thank you for all your kind helps in the lab. Roel and Mies, from the Department of Pharmaceutics; you both were always there when I needed help in the lab. Thank you for being so kind and helpful. My former roommates Anne, Klara, Roderick, Marvin, and Yingxin, thank you all for the nice moments we had together. I wish you all success.

Mithila, Boris, Milena, Eitan, Karla, Yolanda, Dipti, Xingxiu, Funso, Ankit, Bo, Andhyk, Salinthip, Aida, Boning, it was always nice to chat and have coffee breaks with you.

To the following friends who helped me and Kamal a lot since we moved to The Netherlands and created wonderful memories for us: Arie, Mazda, Farshad, Soheil and Nafiseh, Ali and Mehrnoosh, Neda S and Yaser, Neda K, Negar, Mahnaz, Nazila, and Frederik and Charlotte, Thank you all!

Irati and Rowan, we shared wonderful moments together. I am so happy to have such nice friends in my life. Laurens, Ines and their lovely boys Olmo and Duco. Spending time with you was always a pleasure. Nazmiye, getting to know you was one of the best things that happened to me in The Netherlands. When we first met, I could not imagine you will become one of my closest friends. I really enjoy every moment we spend together.

To my brother Sina and his wife Setareh. Words are not good enough to thank my brother for all he has done for me. Throughout our childhood, I always remember you protecting me, although being only 1.5 years older than me. Your helps and advice always made my life better and I am very grateful to have such a kind and supportive brother. My lovely Setareh who came to our family about 4 years ago and made it 
more beautiful. I miss us walking in the malls in Vancouver :-). My lovely niece, I am counting days to hug you baby girl!

To my Mom and Dad, I never forget everything you gave up in your life in order to help me achieve my goals. I am deeply grateful to have such supportive parents and I miss being close to you so much. From the beginning of my PhD, I was thinking it will come a day that I invite you to The Netherlands to attend my promotion. It's a terrible pity that the COVID pandemic made this impossible.

Last but not least, my husband and my best friend, Kamal. My life changed when we started our side project in 2007 . We were always together along the way and managed to solve every problem we encountered in our life. I am always fascinated by your kindness, patience and creativity which makes me feel the luckiest woman in the world $(-)$. Leaving my parents and moving to The Netherlands was the most difficult decision I made in my life but having you by my side gave me the courage and confidence to go through with it. You definitely had a great impact on my scientific and non-scientific achievements.

\section{Thank you!}

\section{Dank U!}




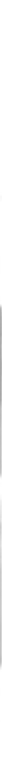

(6)

trat C.

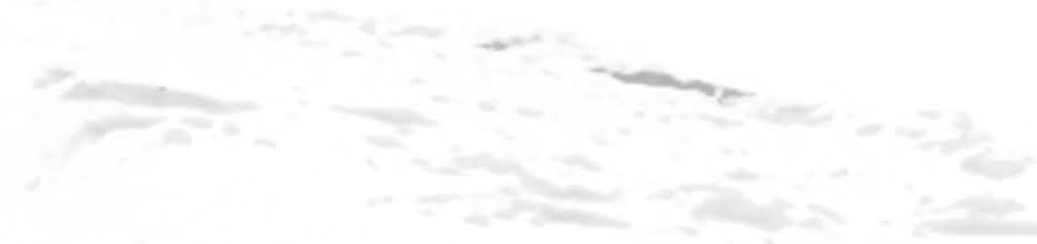

-

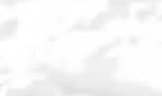

$-7$ 